\title{
Entwicklung und Determinanten von \\ Geschlechtsunterschieden in der Lesekompetenz \\ bei Jugendlichen und Erwachsenen
}

\author{
Inaugural-Dissertation \\ in der Fakultät Humanwissenschaften \\ der Otto-Friedrich-Universität Bamberg
}

\author{
vorgelegt von \\ Kathrin Susanne Thums \\ geboren in Nürnberg
}

Bamberg, den 18. Dezember 2020 
Dieses Werk ist als freie Onlineversion über das Forschungsinformationssystem (FIS; https://fis.uni-bamberg.de) der Universität Bamberg erreichbar. Das Werk steht unter der CC-Lizenz CC-BY.

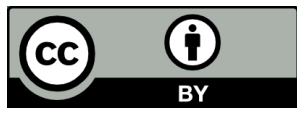

URN: urn:nbn:de:bvb:473-irb-505554

DOI: https://doi.org/10.20378/irb-50555

Tag der mündlichen Prüfung: 20.04.2021

Dekan: Prof. Dr. Jörg Wolstein

Betreuerin: Dr. Ilka Wolter

Weitere Gutachterin: Prof. Dr. Cordula Artelt 


\section{Inhaltsverzeichnis}

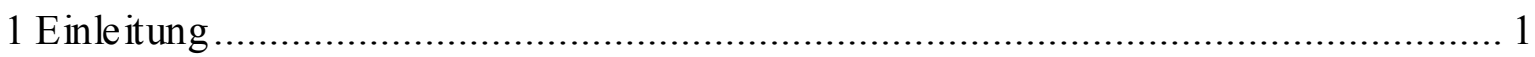

2 Lesekompetenz als Gegenstand der Untersuchungen ......................................... 6

3 Entwicklung von Lesekompetenzunterschieden zw ischen Frauen und Männern ............10

4 Determinanten für die Lesekompetenz von Frauen und Männern ..............................17

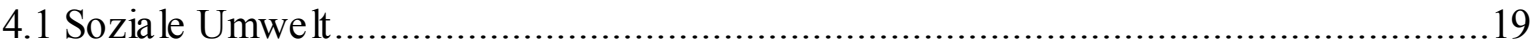

4.2 Merkmale der Lesenden .............................................................................22

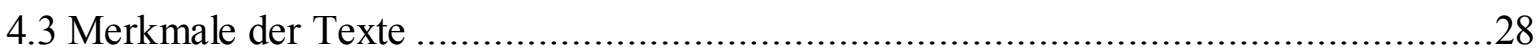

5 Problemstellungen und Forschungsfragen der vorliegenden Arbeit .............................34

5.1 Forschungsfrage 1: Lesekompetenzunterschiede bei jungen Erwachsenen ...................34

5.2 Forschungsfrage 2: Merkmal auf Seiten der Lesenden ...........................................36

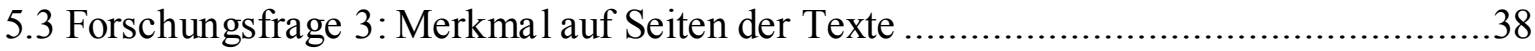

6 Darstellung der zentra len Befunde der einzelnen Beiträge ......................................41

6.1 Beitrag 1: When do gender differences in reading competence diminish? A longitudinal study of reading competence of women and men from adolescence to young

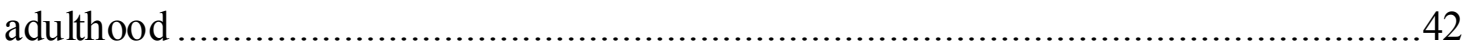

6.1.1 Hypothesen ............................................................................... 42

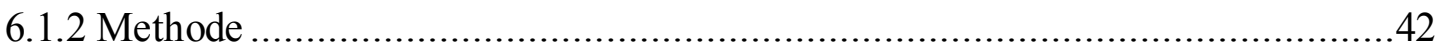

6.1.3 Zentrale Befunde .........................................................................44

6.2 Beitrag 2: Reading for entertainment or information reception? Gender differences in reading preferences and their impact on text-type-specific reading competences in

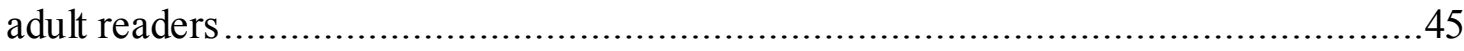

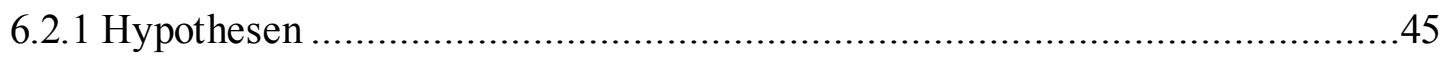

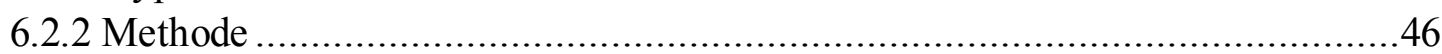

6.2.3 Zentrale Befunde .......................................................................4 48

6.3 Beitrag 3: The impact of gender-stereotypical text contents on reading competences in

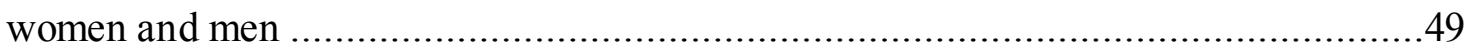

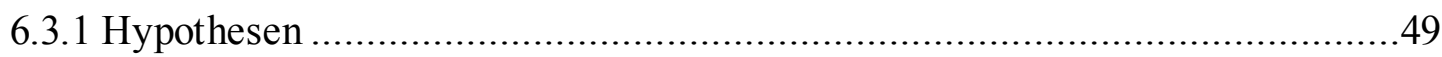

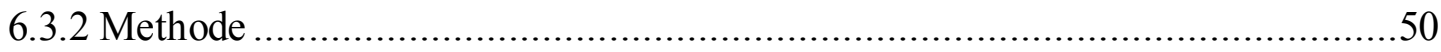

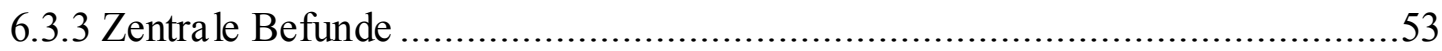

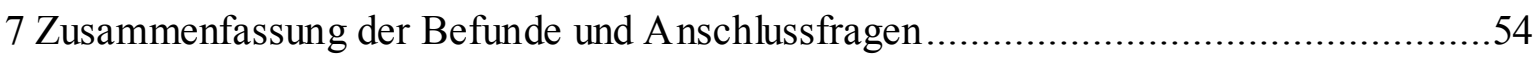

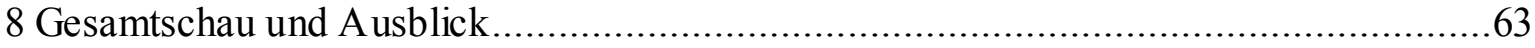

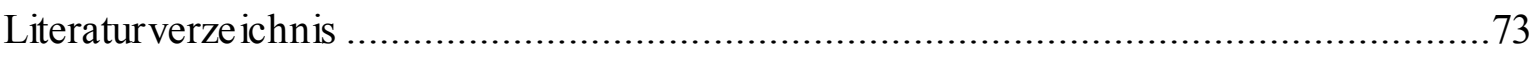

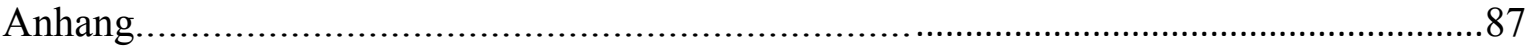

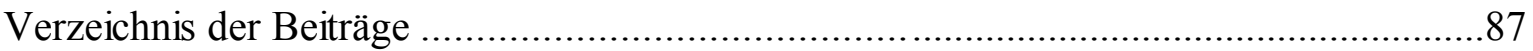




\section{Abbildungsverzeichnis}

Abbildung 1: Determinanten von Lesekompetenz .......................................................18 


\section{Einleitung}

Das Lesen von Texten ist ein wichtiger Bestandteil im gesamten Lebensverlauf fast aller Menschen. $\mathrm{Zu}$ vielen Gelegenheiten im privaten und beruflichen Alltag, in Bildungseinrichtungen oder den Medien werden kürzere oder längere, einfachere oder komplexere Texte gelesen. Je nach Anlass begegnet den Leserinnen und Lesern in ihrer täglichen Lektüre eine große Fülle an Themen, Textsorten und Darstellungsformen. Auch wenn sich Leseanlässe und -anforderungen in einer digitalen Welt verändern, bleibt das Lesen von Texten dennoch ein wichtiger Teil des Alltags, um Informationen aufzunehmen, sich zu unterhalten oder sich mit anderen über das Gelesene auszutauschen (z. B. Christmann \& Groeben, 1999; Groeben, 2004; Naumann, Artelt, Schneider \& Stanat, 2010).

Aus diesen Gründen wird der kompetente Umgang mit geschriebener Sprache als Schlüsselkompetenz bezeichnet. Eine Schlüsselkompetenz dient dazu die unterschiedlichsten Anforderungen des Alltags, der Arbeit sowie des sozialen Lebens in modernen Gesellschaften bewältigen zu können (Tippelt, 2018). Laut dem Europäischen Referenzrahmen sind Schlüsselkompetenzen „diejenigen Kompetenzen, die alle Menschen für ihre persönliche Entfaltung und Entwicklung, Beschäftigung, soziale Inklusion und aktive Bürgerschaft benötigen“ (Europäische Kommission, 2018, S. 7). Mögliche individuelle Unterschiede in der Schlüsselkompetenz Lesen haben nicht nur einen Einfluss auf das Individuum selbst, sondern haben ebenso wirtschaftliche sowie sozial gesamtgesellschaftliche Auswirkungen (Tippelt, 2018; Weinert et al., 2011).

Sowohl innerhalb der Wissenschaft als auch in der Öffentlichkeit sind Unterschiede in der Lesekompetenz zwischen Personengruppen und daraus resultierende Konsequenzen wiederkehrend Teil von Diskussionen ${ }^{1}$. Durch einen Nachteil im Erwerb der Lesekompetenz können bestehende Unterschiede in dieser weiter verfestigt und Ungleichheiten verstärkt werden. Eine Benachteiligung beim Erlernen und bei der Weiterentwicklung der Lesekompetenz von unterschiedlichen Gruppen kann gleichzeitig auch zu

\footnotetext{
Ergebnisse $\mathrm{zu}$ Lesekompetenzunterschieden verschiedener Personengruppen: Personen mit Migrationshintergrund: z. B. Becker \& McElvany, 2018; Berendes et al., 2018. Erläuterungen des Zusammenhangs von Lesekompetenzund Schulform: z. B. Nagy, Retels dorf, Gold hammer, Schiepe-Tiska \& Lüdtke, 2017; Retelsdorf, Becker, Köller \& Möller, 2012; Weis, Müller et al., 2019. Befunde zu gering literalisierten Erwachsenen: z. B. Grotlüschen, Mallows, Reder \& Sabatini, 2016; Vágvölgyi, Coldea, Dresler, Schrader \& Nuerk, 2016.
} 
Bildungsungleichheiten, zum Beispiel beim Erwerb von Bildungsabschlüssen, führen oder zu erschwerten Entwicklungsmöglichkeiten hinsichtlich einer erfolgreichen Teilhabe an der Gesellschaft. Folglich ist das Individuum in seiner Mitbestimmung, Weiterentwicklung und Entfaltung der eigenen Möglichkeiten, aber auch an der Partizipation in der Gesellschaft eingeschränkt (z. B. Organization for Economic Co-operation and Development [OECD], 1999; Sälzer \& Reiss, 2016; Tippelt, 2018; Weinert et al., 2011). Für einzelne Personengruppen ist es aus diesen Gründen ist es sinnvoll, Faktoren zu identifizieren, welche die Lesekompetenz bedeutend beeinflussen. Dadurch wird die Möglichkeit gegeben, Benachteiligungen bereits frühzeitig zu erkennen, um adäquate Hilfen und Förderung bereitzustellen. Gleichzeitig besteht eine realistisch Möglichkeit Chancenungleichheit zu reduzieren.

Ein individuelles Merkmal, nach dem Unterschiede in der Lesekompetenz untersucht werden, ist das Geschlecht. Individuelle Stärken und Schwächen in der Lesekompetenz können bei Mädchen/Frauen bzw. Jungen/Männern² identifiziert werden. Es liegen viele Befunde vor, die Geschlechtsunterschiede in der Lesekompetenz bestätigen (z. B. Berendes et al., 2018; Chiu \& McBride-Chang, 2006; McElvany, Kessels, Schwabe \& Kasper, 2017; Solheim \& Lundetræ, 2017; Weis, Doroganova et al., 2019). Auch wenn die gefundenen Lesekompetenzunterschiede zwischen Mädchen/Frauen bzw. Jungen/Männer statistisch gesehen im Mittel nur als kleine Effekte beschrieben werden können, wäre es dennoch erstrebenswert, wenn diese Unterschiede unter dem Aspekt der Chancengleichheit nicht vorhanden wären, bzw. so gering wie möglich ausfallen würden. Daher ist es sinnvoll, unterschiedliche Entwicklungsverläufe und mögliche Determinanten in der Lesekompetenz von Mädchen/Frauen und Jungen/Männern genauer zu betrachten. Aus den resultierenden Ergebnissen können wiederum Rückschlüsse gezogen werden, zu welchen Zeitpunkten im Entwicklungsverlauf Unterschiede begünstigt werden und welche Bedingungsfaktoren zu Diskrepanzen beitragen, bzw. diese auch beheben.

\footnotetext{
${ }^{2}$ In der vorliegenden Arbeit wird das Geschlecht als dichotome Variable in männlich und weiblich unterteilt. Der Autorin ist bewusst, dass eine weitaus größere Vielfalt an Geschlechtern vorliegen kann, welche sowohl biologis che als auch soziale Aspekte beinhalten kann.
} 
Ein, für die Entwicklung der Lesekompetenz, elementarer Abschnitt im Lebensverlauf ist der Eintritt in das formale Schulsystem. Mit Beginn der Grundschulzeit kommt es zu einer raschen Entwicklung der Lesekompetenz (Alexander, 2005; Chall, 1983; Philipp, 2011; Schmidt-Hertha, 2018). Dies belegen eine Vielzahl von Forschungsarbeiten zur Entwicklung der Lesekompetenz im Kindes- und Jugendalter (z. B. Cameron, Grimm, Steele, Castro-Schilo \& Grissmer, 2015; McElvany, Kortenbruck \& Becker, 2008; Retelsdorf, Köller \& Möller, 2011; Robinson \& Lubienski, 2011).

Diese Arbeiten und weitere andere Studien stellten einen Unterschied in der Lesekompetenz von Jungen und Mädchen fest. So wurde durch die Befundlage wiederholt gezeigt, dass Mädchen und junge Frauen im Durchschnitt eine höhere Lesekompetenz erzielen als Jungen und junge Männer (z. B. Berendes et al., 2018; McElvany et al., 2017; Solheim \& Lundetræ, 2017; Weis, Doroganova et al., 2019). Ergebnisse zur Lesekompetenz von Erwachsenen deuten jedoch daraufhin, dass sich im Erwachsenenalter zwischen Frauen und Männern der Unterschied in deren Lesekompetenz im Allgemeinen nivelliert (u. a. OECD, 2016). Allerdings liegen für differenzierte Aussagen zu Stärken und Schwächen junger Frauen und Männer in der Lesekompetenz und deren Entwicklung nach der Sekundarstufe II (Nagy et al., 2017) sowie im Erwachsenenalter (u. a. OECD, 2016) nur wenige Befunde vor. Es kann also im Hinblick auf die Entwicklung der Lesekompetenz über das Kindes- und Jugendalter hinaus, noch kein eindeutiger Verlauf dieser für Frauen und Männer beschrieben werden. Um zukünftig mehr zur Entwicklung der Lesekompetenz über längere Lebensphasen hinweg zu erfahren, knüpft diese Arbeit an die bisherige Forschung zur Lesekompetenz im Kindes- und Jugendalter an und untersucht die Lesekompetenz von jungen Frauen und Männern im Übergang zwischen der Sekundarschulbildung und der Zeit nach der Schule. Auf diesem Weg leistet die vorliegende Forschung einen Beitrag zur Analyse der Weiterentwicklung der Lesekompetenz von Frauen und Männern nach dem Ende der Schulzeit.

Als mögliche Erklärungen für die Unterschiede in der Lesekompetenz von Mädchen/Frauen und Jungen/Männern wurden in der Forschung verschiedene Faktoren, wie kognitive Unterschiede oder die Feminisierung von Schule und Lerngelegenheiten diskutiert (u. a. Hannover \& Kessels, 2011; Heyder \& Kessels, 2013; Solheim \& Lundetræ, 2017). Allerdings lassen sich Geschlechtsunterschiede in der Lesekompetenz weitgehend durch sozial-kulturelle Konstruktionen von Geschlecht und Erwartungen der Umwelt an Mädchen/Frauen und Jungen/Männern hinsichtlich der Schlüsselkompetenz Lesen 
(u. a. Heyder \& Kessels, 2013; Steffens \& Jelenec, 2011) sowie motivational-behaviorale Einstellungen zum Lesen (u. a. McElvany et al., 2017; Solheim \& Lundetræ, 2017; Stanat \& Kunter, 2002) erklären. An dieses Ergebnis schließt die vorliegende Forschung zur Erklärung von Geschlechtsunterschieden an und fokussiert insbesondere Determinanten der Lesekompetenz auf der motivationalen und sozialen Ebene. Die Systematisierung der Determinanten der Lesekompetenz erfolgt dabei durch eine Unterteilung von möglichen Einflussfaktoren: Zum einen wird die soziale Umwelt als umspannender Rahmen mittels der Übernahme und Weitergabe von Stereotypen hinsichtlich des Lesens in die Betrachtung einbezogen. Zum anderen können Unterschiede in der Lesekompetenz durch Merkmale der Lesenden (z. B. Vorwissen, Motivation oder Lesestrategien) und über Einflussfaktoren auf Seiten der gelesenen Texte (z. B. Leseanforderungen oder Merkmale der Texte) erklärt werden.

Auf dieser Grundlage hat die vorliegende Arbeit zwei Ziele: Zunächst soll die Entwicklung von Lesekompetenzunterschieden im Lebensverlauf zwischen jungen Frauen und Männern beschrieben werden. Dafür soll geklärt werden, wie sich Geschlechtsunterschiede in der Lesekompetenz im jungen Erwachsenenalter im Übergang vom institutionellen Lernkontext der Schule bis in die Zeit nach der Schule weiterentwickeln. Da es bisher wenige Längsschnitt-Befunde zum Verlauf von Geschlechtsunterschieden in der Lesekompetenz von jungen Erwachsenen gibt, versucht die vorliegende Arbeit diese Forschungslücke zu schließen.

Als weiteres Ziel werden im Zuge der Arbeit relevante Determinanten betrachtet, die Geschlechtsunterschiede in der Lesekompetenz erklären können. Diese Determinanten sind in einen größeren Kontext von sozialen Einflussfaktoren eingebettet. Über die soziale Umwelt werden Einstellungen und Erfahrungen hinsichtlich des Lesens vermittelt und weitergegeben. Aus diesem Grund wird die soziale Umwelt als Prädiktor für die Lesekompetenz herangezogen. Darüber hinaus wird der Einfluss von personenspezifischen (Leseinteressen und -präferenzen) und textspezifischen (Textsorten, Textmerkmale) Determinanten auf die Lesekompetenz von Frauen und Männern untersucht. 
Unter Berücksichtigung der vorangehend beschriebenen Zielsetzungen gliedert sich die Arbeit wie folgt: Zunächst wird die Lesekompetenz als zentrales Konstrukt der Arbeit genauer definiert (Kapitel 2), bevor dann im Speziellen auf die Entwicklungsverläufe der Lesekompetenz bei Frauen und Männer eingegangen wird (Kapitel 3). Darauf folgend werden Determinanten der Lesekompetenz umfassend erläutert: Als erstes wird dabei der Einfluss der sozialen Umwelt auf die Lesekompetenz dargestellt (Kapitel 4.1) sowie anschließend die Merkmale der Lesenden und deren Einflussfaktoren auf die Lesekompetenz (Kapitel 4.2) und die textspezifischen Determinanten (Kapitel 4.3).

Nach der theoretischen Einbettung werden drei Forschungsfragen abgeleitet (Kapitel 5), um die daran anknüpfenden Forschungsarbeiten und ihre zentralen Befunde genauer vorzustellen (Kapitel 6).

Abschließend werden die Ergebnisse aus den einzelnen Beiträgen analysiert und mögliche Anschlussfragestellungen aus diesen abgeleitet (Kapitel 7). Anknüpfend an die Einzelergebnisse der jeweiligen Beiträge werden eine Gesamtschau sowie ein Ausblick zur Fragestellung der Lesekompetenzunterschiede bei Frauen und Männern gegeben und diskutiert (Kapitel 8). 


\section{Lesekompetenzals Gegenstand der Untersuchungen}

Die Fähigkeit, geschriebene Sprache zu lesen und zu verstehen, ist eine zentrale Schlüsselkompetenz und wird als Lesekompetenz bezeichnet (u. a. Europäische Kommission, 2018; Lenhard \& Artelt, 2009; Müller \& Richter, 2014). Im Folgenden wird sich spezifisch mit der Lesekompetenz auseinandergesetzt und diese genauer definiert, um eine konkrete wissenschaftliche Positionierung für die vorliegende Fragestellung vornehmen zu können.

Kompetenzen sind kognitive Fertigkeiten, die in formalen, nichtformalen und informellen Umgebungen erlernt werden und sich über verschiedene Lebensphasen hinweg entwickeln (Europäische Kommission, 2018; Weinert, 2001). In Abgrenzung zur Intelligenz ist zu betonen, dass Kompetenzen erlernbar und veränderbar sind sowie anhand spezieller Anforderungen, Aufgaben und Situationen erworben werden können. Somit ist der Prozess der Entwicklung und Veränderung von Kompetenzen nie abgeschlossen und setzt sich in unterschiedlicher Intensität über die gesamte Lebensspanne hinweg fort (Klieme \& Hartig, 2008; Schmidt-Hertha, 2018). Der Kompetenzerwerb und die weitere Entwicklung von Kompetenzen hängen stark vom Individuum und dessen motivationalen und handlungsbezogenen Merkmalen ab. Daher kommt es für das Erlernen, die Entwicklung und Anwendung von Kompetenzen auch immer auf Motivation, Verstehen, Wissen, Können und Handeln der einzelnen Person an (Weinert, 2001).

Genauso ist die Lesekompetenz als ein vielschichtiges, lern- sowie trainierbares Fertigkeitskonstrukt zu verstehen, bestehend aus mehreren interagierenden Teilfähigke iten (Lenhard \& Artelt, 2009; Müller \& Richter, 2014). Diese Teilfähigkeiten, wie zum Beispiel der Wortschatz, das Vorwissen oder die Arbeitsgedächtniskapazität sind nicht nur spezifis ch für das Lesen notwendig, sondern beeinflussen ebenso andere sprachliche Leistungen wie das Hörverstehen oder die Textproduktion (Lenhard \& Artelt, 2009). Auch auf Grundlage dieser Vielschichtigkeit lässt sich aus der Literatur keine einheitliche Definition für Lesekompetenz ableiten. Je nach Perspektive, Fragestellung und Modellierung werden unterschiedliche Schwerpunkte gesetzt und die Definition enger oder weiter gefasst (Müller \& Richter, 2014; Philipp, 2018). Nach Graesser, Singer und Trabasso (1994) definiert Lesekompetenz nicht nur das reine Entziffern von Buchstaben, Wörtern und Sätzen. Vielmehr impliziert diese die aktive Auseinandersetzung mit dem Geschriebenen, um daraus 
auf den Text bezogene, aber auch darüber hinaus globale Zusammenhänge über den gelesenen Text hinaus herzustellen.

Ein weiteres relevantes Konzept, das in Zusammenhang mit der Lesekompetenz steht, ist die Literacy-Konzeption (z. B. Lenhard, 2019; OECD, 1999). In dieser wird hervorgehoben, dass das Lesen von Texten in modernen Gesellschaften eine funktionale Grundbildung für eine adäquate, alltägliche Lebensführung darstellt (Sälzer \& Reiss, 2016). Dabei werden relevante Kompetenzen für das Leben in modernen Gesellschaften und Kulturen, wie eben die Lesekompetenz, im Sinne einer Schlüsselkompetenz verstanden (Europäische Kommission, 2018). Durch die aktive und reflektierte Anwendung des Lesens von Texten, ist die Teilhabe an einer Kultur möglich und die Anschlussfähigkeit im Sinne eines beständigen Weiterlernens, nicht nur in der Schule, sondern über die Lebensspanne hinweg in verschiedenen Inhaltsbereichen gegeben. Damit wird das Lesen von Texten und der kompetente Umgang mit gelesenen Inhalten auch $\mathrm{zu}$ einem Instrument, um sich andere Kompetenzbereiche und Wissensgebiete $\mathrm{zu}$ erschließen (Artelt, Stanat, Schneider \& Schiefele, 2001; Europäische Kommission, 2018; Naumann et al., 2010; OECD, 1999).

In einer enger gefassten Definition beinhaltet Lesen die hierarchieniedrigere Lesefertigkeit (Buchstaben- und Satzebene) sowie das hierarchiehöhere Leseverstehen. Diese kognitionspsychologische Perspektive auf das Lesen ist überwiegend an den beschreibbaren Prozessen des Lesens interessiert (Müller \& Richter, 2014; Philipp, 2018). Lesen wird dabei als eine kognitive Aktivität aus mehreren hierarchischen Teilprozessen auf Wort-, Satz- und Textebene verstanden. Die drei aufeinander aufbauenden mentalen Modelle der Textrepräsentation setzen sich wie folgt zusammen:

a) hierarchieniedrigere Prozesse: fehlerfreies und schnelles Rekodieren und Dekodieren von Buchstaben und Wörtern, lokale Kohärenzbildung, um Beziehungen und Bedeutungen sowohl zwischen Wörtern als auch zwischen Sätzen herzustellen (Propositionsbildung). Diese Prozesse laufen meist automatisiert ab und werden kaum durch bewusste Aufmerksamkeit gesteuert (Kintsch, 1998; Lenhard, 2019; Müller \& Richter, 2014; Richter \& Christmann, 2009; van Dijk \& Kintsch, 1983). 
b) hierarchiehohe Prozesse: satzübergreifende Integration von Sätzen zu ganzen Bedeutungseinheiten, um darauf aufbauend globalere Kohärenz zu bilden. Es werden über den Text hinaus Schlussfolgerungen gezogen (Inferenzbildung; Kintsch, 1998; Lenhard, 2019; Müller \& Richter, 2014; Richter \& Christmann, 2009; van Dijk \& Kintsch, 1983).

c) Situationsmode ll: aufbauend auf den vorhergehenden Prozessen, entsteht beim Leser oder der Leserin eine mentale Repräsentation der im Text beschriebenen Sachverhalte. Durch eigenes Vorwissen und Schlussfolgerungen werden die Inhalte des Textes in eigenen Worten zusammengefasst (Kintsch, 1998; Lenhard, 2019; Müller \& Richter, 2014; Richter \& Christmann, 2009; van Dijk \& Kintsch, 1983).

Hervorzuheben ist, dass die einzelnen Teilprozesse trotz hierarchisierender Bezeichnungen nicht im Sinne einer Wertung oder strikten Abfolge zu verstehen sind. Aus interaktionistischer Perspektive stehen die Teilprozesse auf den unterschiedlichen Ebenen miteinander in wechselseitiger Beziehung und laufen zeitlich parallel und nicht sequentiell nacheinander ab (Christmann \& Groeben, 1999; Richter \& Christmann, 2009; van Dijk \& Kintsch, 1983). Individuelle Unterschiede zwischen den Lesenden kommen durch eine unterschiedliche Effizienz in den einzelnen kognitiven Prozessen zustande, beispielsweise in der Worterkennung oder der Kohärenzbildung zwischen verschiedenen Informationen (Müller \& Richter, 2014).

Im Gegensatz dazu wird aus differenziell psychologischer Sichtweise eine weiter gefasste Perspektive auf das Lesen eingenommen. Hier steht vor allem das resultierende Produkt, also die Qualität der individuellen Textrepräsentation, im Vordergrund. Bei der Erfassung des Leseverständnisses handelt es sich nicht um einzelne kognitive Prozesse und deren Zusammenspiel sowie deren mögliche Defizite, sondern um das Merken und Verstehen der Informationen aus den Texten. Beim Leseverständnis soll aus dem Geschriebenen der Sinngehalt entnommen werden, damit die Leserin oder der Leser mit dem Inhalt des Textes arbeiten kann. Es werden die Qualität der Textrepräsentation für unterschiedliche Textsorten sowie Anforderungsniveaus in Form eines Lesekompetenztests erfasst (Müller \& Richter, 2014). Auf Basis des Arbeitens mit einem Text, häufig in Form von Beantwortung von textbezogenen Fragen, wird das Produkt der Lesekompetenz gemessen. Anhand der 
Leseleistung können Unterschiede zwischen Personengruppen messbar und diagnostizierbar gemacht werden (Müller \& Richter, 2014; Philipp, 2018). Vor allem in der empirischen Bildungsforschung und Large-Scale Assessments ist die produktorientierte Erfassung der Lesekompetenz üblich (z. B. PIRLS: Mullis, Martin, Foy \& Hopper, 2017; PISA: OECD, 1999; NEPS: Weinert et al., 2011). In diesen bekommen die Studienteilnehmerinnen und teilnehmer Texte mit variierender Schwierigkeit aus verschiedenen Textsorten (z. B. literarische Texte, Sachtexte) und Themengebieten vorgelegt und müssen dazu Verständnisfragen unterschiedlicher Anforderungen und Komplexität bearbeiten (z. B. Informationen im Text suchen und extrahieren, Interpretationen zum Text umsetzen sowie Schlussfolgerungen, Reflektionen und Bewertungen des Textinhaltes vornehmen). Auf Grundlage der richtig beantworteten Aufgaben werden im Anschluss Rückschlüsse auf die Lesekompetenz der Personen gezogen (Becker-Mrotzek et al., 2019; Müller \& Richter, 2014; OECD, 2016).

Wesentlich ist dabei, dass der beschreibbare Prozess und das messbare Produkt des Lesens nicht getrennt voneinander zu betrachten sind. Denn ohne den kognitiven Prozess des Lesens gibt es keine Fähigkeit, die gemessen werden kann. Ebenso bedarf es eines nötigen Prozesses, um ein Leseverständnisprodukt zu erhalten. Somit greifen Prozess und Produkt des Lesens stets ineinander (Philipp, 2018; Rost \& Buch, 2018).

Auf Grundlage der vorangestellten Ausführung zur Lesekompetenz ist nun eine genauere wissenschaftliche Einordnung der vorliegenden Fragestellungen möglich. Der im Folgenden benutzte Lesekompetenz-Begriff orientiert sich an der differenziell-psychologischen Perspektive. In der vorliegenden Arbeit wird infolgedessen das Produkt des Lesens in den Blick genommen und weniger die einzelnen kognitiven Anforderungen. Außerdem werden differenzielle Effekte für Personengruppen (hier Frauen und Männer) betrachtet und diese beiden Gruppen hinsichtlich ihrer Kompetenz miteinander verglichen. 


\section{Entwicklung von Lesekompetenzunterschieden zwischen Frauen und Männern}

Die Ergebnisse der Lesekompetenzmessung aus PISA 2018 (Programme for International Student Assessment) zeigten, dass sich im OECD-Durchschnitt $23 \%$ (für Deutschland $21 \%$ ) und somit fast ein Viertel der 15-jährigen Schülerinnen und Schüler auf der untersten von acht möglichen Kompetenzstufen befinden. Die Leserinnen und Leser können demzufolge zwar das Hauptthema des Textes erschließen, haben jedoch Schwierigkeiten Kohärenz herzustellen und im Text verteilte Informationen aufzufinden (Weis, Doroganova et al., 2019). In den Daten von PIAAC (Programme for the International Assessment of Adult Competencies) zeigt sich für die Lesekompetenz von Erwachsenen (16 - 64 Jahren), dass im OECD-Durchschnitt rund $16 \%$ der Teilnehmenden (für Deutschland $18 \%$ ) nicht in der Lage sind, kurze Texte auf Basis eines einfachen Grundwortschatzes zu lesen (OECD, 2016). Für diese Personengruppen bestehen, wie in der Einleitung beschrieben, folglich Benachteiligungen und Einschränkungen im alltäglichen Leben bezüglich des Erreichens von Schulabschlüssen, der Berufswahl sowie der generellen Teilhabe am gesellschaftlichen Leben (z. B. OECD, 1999; Sälzer \& Reiss, 2016; Tippelt, 2018; Weinert et al., 2011). Um präzise differenzieren zu können, welche Personen von einer niedrigen Lesekompetenz betroffen sind, ist es notwendig die Gesamtgruppe nach vulnerablen Personengruppen zu unterscheiden (z. B. nach soziökonomischem Status, sprachlichem Hintergrund oder besuchter Schulform).

Ein weiteres individuelles Merkmal, welchem eine große Relevanz für die Lesekompetenz zukommt, ist das Geschlecht einer Person. Auf Basis der Ergebnisse der Meta-Analyse von Hyde $(2005,2014)$ kann geschlussfolgert werden, dass es zwischen Frauen und Männern mehr Ähnlichkeiten und Überschneidungen in verschiedenen Konstrukten gibt als Unterschiede. Die Autorin zeigte dabei auf, dass überwiegend kleine bis keine Unterschiede in den insgesamt 46 betrachteten Studien nachweisbar waren (,gender similarities hypothesis"). Allerdings konnte Hyde (2014) in ihrer Darstellung gerade bei sprachlichen Fertigkeiten (Leseverständnis, Wortschatz und Textproduktion) je nach Konstrukt unterschiedlich hohe Effektstärken für Frauen und Männer identifizieren. Trotz der allgemeinen Annahme der Ähnlichkeit von Männern und Frauen, stellte Hyde (2014) besonders im Leseverständnis kleine Effekte $(0.11<d<0.35)$ zu Gunsten der Frauen fest. 
Diese Unterschiede in der Lesekompetenz von Mädchen/Frauen und Jungen/Männern zeigen sich ebenso kontinuierlich in anderen Studien (z. B. Berendes et al., 2018; Solheim \& Lundetræ, 2017; Weis, Doroganova et al., 2019). Im Folgenden sollen bisher bekannte Ergebnisse zu Geschlechtsunterschieden in der Lesekompetenz genauer dargestellt werden. Dabei wird eine lebenslange Perspektive auf die Entwicklung der Lesekompetenz eingenommen und Ergebnisse aus verschiedenen Altersbereichen aufgezeigt, um die Entwicklung von Geschlechtsunterschieden in der Lesekompetenz herauszuarbeiten.

Hinsichtlich der Beschreibung der Lesekompetenz von Mädchen/Frauen und Jungen/Männern sind drei divergente Verläufe in der Entwicklung der Lesekompetenz möglich:

a) Kompetenzunterschiede zwischen den Geschlechtern bleiben über die Zeit hinweg stabil („Karawaneneffekt“ bzw. stability growth; Pfost, Hattie, Dörfler \& Artelt, 2014).

b) Die Unterschiede zwischen Frauen und Männer werden größer (,Matthäuseffekt“ bzw. cumulative growth; Baumert, Nagy \& Lehmann, 2012; Pfost et al., 2014).

c) Die Unterschiede zwischen den Geschlechtern verringern sich bzw. verschwinden im Laufe der Zeit, da eine Gruppe aufholt bzw. den Unterschied kompensieren kann („Kompensations-/Aufholeffekt““ bzw. compensatory growth; Baumert et al., 2012; Berendes et al., 2018; Pfost et al., 2014).

Die vorliegende Darstellung vorhandener Ergebnisse hinsichtlich der Geschlechtsunterschiede in der Lesekompetenz beginnt mit dem Ende der Grundschulzeit. Dies ist darin begründet, dass im Altersbereich zwischen neun und dreizehn Jahren davon auszugehen ist, dass sich ein Wechsel in der Art und Weise sowie im Zweck des Lesens vollzieht. Ab diesem Zeitpunkt gewinnen komplexere Lese- und Verstehensprozesse auf der Textebene für die Lesenden an Bedeutung (Klicpera \& Gasteiger-Klicpera, 1993). Vor Schulbeginn und in den ersten Schuljahren stehen das Erlernen von basalen Lesefähigkeiten und der Schriftspracherwerb im Vordergrund. Mit dem Ende der Grundschulzeit und dem Beginn der Sekundarstufe kann schließlich das Gelesene zunehmend für Lernprozesse genutzt werden. $\mathrm{Zu}$ diesem Zeitpunkt ist der Leseprozess soweit automatisiert, dass 
genügend Kapazitäten vorhanden sind, um unter anderem Lesestrategien anzuwenden, Vorwissen $\mathrm{zu}$ aktivieren und eine Kohärenz $\mathrm{zu}$ bereits bekannten Themengebieten herzustellen. Infolgedessen vermittelt das Gelesene neue Ideen, mehr Wissen und neue Erfahrungen (Alexander, 2005; Becker, McElvany \& Kortenbruck, 2010; Chall, 1983; Pfost \& Artelt, 2013).

Für die Lesekompetenz von Schülerinnen und Schüler in der Grundschule (4. Klasse) konnte mit den Querschnitts-Ergebnissen aus der PIRLS-Studie (Progress in International Reading Literacy Study) in der letzten internationalen Erhebung aus dem Jahr 2016 gezeigt werden, dass in nahezu allen 50 teilnehmenden Ländern Mädchen im Durchschnitt eine höhere Lesekompetenz erzielen als Jungen. Der internationale Durchschnitt der Lesekompetenz lag für Mädchen bei $M=520$ Punkten $(S E=0.4)$ und für Jungen bei $M=501$ Punkten $(S E=0.5)$. Nur in Macau und Portugal bestand kein signifikanter Unterschied hinsichtlich der Lesekompetenz zwischen Jungen und Mädchen (Mullis et al., 2017). In der deutschen PIRLS-Stichprobe, in Deutschland unter dem Namen IGLU-Studie (Internationale Grundschul-Lese-Untersuchung) bekannt, zeigten sich ähnliche Unterschiede wie im internationale Gesamtvergleich in der Lesekompetenz zwischen Jungen und Mädchen. So verdeutlichen die Ergebnisse, dass Mädchen im Durchschnitt eine höhere Leseleistung erzielen (11 Kompetenz-Punkte), auch wenn dies nur als kleiner Effekt zu bewerten ist $(d=0.10)$. Darüber hinaus ist festzustellen, dass in Relation $\mathrm{zu}$ vorangegangenen IGLUErgebnissen im Zeitverlauf über die jeweiligen Kohorten, keine signifikanten Veränderungen hinsichtlich der Unterschiede in der Lesekompetenz von Jungen und Mädchen zu erkennen sind (McElvany et al., 2017). Der IQB-Bildungstrend (Institut zur Qualitätsentwicklung im Bildungswesen) von 2016 konnte für die deutschen Schülerinnen und Schüler zum Ende der 4. Jahrgangsstufe ebenso Geschlechtsunterschiede in der Lesekompetenz mit einem deutlichen Kompetenzvorteil für Mädchen im Vergleich zu Jungen nachweisen $(d=0.24)$. Die Ergebnissen des IQB-Bildungstrends verdeutlich darüber hinaus, dass zwischen 2011 und 2016 die Geschlechtsdifferenzen in der Lesekompetenz stabil geblieben sind (Schipolowski, Wittig, Weirich \& Böhme, 2017). Dies könnte implizieren, dass sich die Unterschiede zum Ende der Grundschulze it zwischen Jungen und Mädchen über den Zeitverlauf nicht verändert haben. Mit den Daten von PIRLS/IGLU und des IQB-Bildungstrends ist jedoch der Vergleich im Längsschnitt nur bedingt möglich, da Daten dieser Studien alle im Querschnitt erhoben werden. 
Für die Sekundarstufe I konnte in der Studie von Berendes und Kolleginnen sowie Kollegen (2018) für Schülerinnen und Schüler aus Baden-Württemberg in den Klassenstufen fünf bis acht im Längsschnitt gezeigt werden, dass Jungen zu Beginn der Sekundarstufe I einen niedrigeren Ausgangswert im Leseverständnis haben. Im weiteren Verlauf bis zur 8. Klasse war das Wachstum für Jungen geringer als für Mädchen. Da der Unterschied zwischen den beiden Geschlechtern über vier Messzeitpunkte größer wurde, deuten die Ergebnisse der Studie auf einen Scheren- bzw. Matthäuseffekt zu Ungunsten der Jungen hin (Berendes et al., 2018).

Zum Ende der Pflichtschulzeit in der Sekundarstufe I konnten Ergebnisse der PISA-Studie aus dem Jahr 2018 für die Lesekompetenz 15-jähriger Schülerinnen und Schüler zum wiederholten Male Unterschiede zwischen jungen Männern und Frauen verdeutlichen. In allen vorausgehenden PISA-Erhebungen seit 2000 erreichten junge Frauen signifikant höhere Leseleistungen als die 15-jährigen jungen Männer. Im OECD-Durchschnitt erzielten junge Frauen in PISA 201830 Kompetenz-Punkte mehr als junge Männer. Für Deutschland lag ein Unterschied in der Lesekompetenz von 26 Punkten zwischen den Geschlechtern vor (junge Frauen: $M=512$ Punkte, $S E=3.2$; junge Männer: $M=486 ; S E=3.4 ; \mathrm{OECD}, 2019$; Weis, Doroganova etal., 2019). Dies ist mit einer Effektstärke von $d=0.24$ als kleiner Effekt zu bewerten. Im Trend gibt die OECD (2019) an, dass sich die Unterschiede zwischen jungen Männern und Frauen in den letzten zehn Jahren im Durchschnitt um 10 KompetenzPunkte verringert haben. Allerdings ist bei der Interpretation von Trend-Ergebnissen an dieser Stelle kritisch anzumerken, dass bei PISA 2018 zum zweiten Mal die Erhebung am Computer stattfand und zudem eine Veränderung in der Konzeption der Lesekompetenz erfolgte. Im Zuge des zunehmenden digitalen Lesens und des Umgangs mit digitalen Geräten und Informationsquellen, wurde das Framework der Lesekompetenz im Vergleich zu den bisherigen PISA-Erhebungen angepasst (Becker-Mrotzek et al., 2019). Ebenso wurde die Skalierung der Ergebnisse seit der PISA-Erhebung 2015 wiederholt adaptiert (Heine \& Reiss, 2019). Diese Punkte sollten bei der Interpretation der PISA-Ergebnisse und dem Vergleich über die verschiedenen Zyklen bedacht werden.

Im Vergleich zu den vielfältigen Ergebnissen hinsichtlich der Geschlechtsunterschiede in der Lesekompetenz zu Beginn der Sekundarstufe I, gibt es weniger Befunde am Ende der Sekundarstufe I und der Sekundarstufe II sowie darüber hinaus nach der Schule und im Erwachsenenalter. In einer der wenigen Studien untersuchten Nagy und Kolleginnen sowie 
Kollegen (2017) die Lesekompetenzentwicklung nach der Sekundarstufe I. Hier wurde die Lesekompetenzentwicklung von der 9. zur 10. Jahrgangsstufe mit einer Teilstichprobe von Schülerinnen und Schülern an Gymnasien, Realschulen sowie Gesamtschulen des PISALängsschnitts 2012/2013 betrachtet. Den Geschlechtsunterschied in der Lesekompetenz betreffend, zeigen sich geringe Vorteile für junge Frauen im Lesen im Vergleich zu jungen Männern (Nagy et al., 2017).

In einer weiteren Studie befassten sich De Fraine, Van Damme und Onghena (2007) mit der Kompetenzentwicklung von Schülerinnen und Schülern von der siebten bis zur zwölften Klasse. Hier wurden Geschlechtsunterschiede in verschiedenen leserelevanten Bereichen (Rechtschreiben, Grammatik und Leseverständnis) bei Schülern und Schülerinnen im niederländisch sprechenden Teil von Belgien erhoben. In dieser Studie konnten die Autorin und ihre Kollegen zeigen, dass junge Frauen eine höhere Leistung erzielten als junge Männer. Außerdem konnten gleichzeitig differenzierte Entwicklungsverläufe für junge Männer und Frauen dokumentiert werden. Die teilnehmenden jungen Frauen hatten ein relativ konstantes Wachstum in den sprachlichen Leistungen und verfügten am Ende der Sekundarstufe II über einen Lernzuwachs im Vergleich zum Beginn der Sekundarstufe. Bei den jungen Männern zeigte sich ein anderes Verlaufsmuster: So hatten junge Männer in der siebten Klasse eine höhere Sprachkompetenz (3.53 Logits) als junge Frauen (3.47 Logits). Zwischen der siebten und der neunten Klasse sank jedoch die Leistung der jungen Männer ab, bevor diese bis zur 12. Klasse wieder anstieg. Diese Divergenz in der Entwicklung mündete schließlich im gleichen Niveau der jungen Männer mit dem der jungen Frauen in der 12. Klasse. Die Autorin und die Autoren führten den Abfall der Leistungen der jungen Männer auf einen möglichen Mangel in der Testmotivation zurück. Allerdings ist anzumerken, dass die Studie nicht ausschließlich Lesekompetenz gemessen hat, sondern zudem Grammatik und Rechtschreibung in den Messungen berücksichtigt wurden. Dies könnte den Unterschied im Ausgangsniveau zwischen den Jugendlichen in der siebten Klasse erklären.

Im fortschreitenden Lebensverlauf gibt es wenige Ergebnisse zu Geschlechtsunterschieden in der Lesekompetenz. Die Ergebnisse von PIAAC deuten darauf hin, dass sich keine signifikanten Unterschiede in der Lesekompetenz von Männern und Frauen zwischen 16 - 64 Jahren ergeben (OECD, 2016). In der deutschen PIAAC-Stichprobe erzielten Frauen im Durchschnitt 267 Punkte $(S D=47)$ und Männer im Durchschnitt 272 Punkte $(S D=48)$ 
im Lesen. Vor allem in der jüngeren Altersgruppe (geboren 1988 - 1996) zeigten sich in PIAAC keine signifikanten Geschlechtsunterschiede in der Lesekompetenz (Maehler et al., 2013). Jedoch soll an dieser Stelle zu bedenken gegeben werden, dass die PIAAC-Stichprobe eine große Altersspanne abdeckt. Möglicherweise könnte sich der Erwerb der Lesekompetenz in den letzten Jahrzehnten positiv entwickelt haben und somit in der Stichprobe ein Kohorteneffekt vorliegen.

In einer Gegenüberstellung der Ergebnisse von Dänemark, Finnland, Norwegen und Schweden aus PIRLS 2011, PISA 2009 und den jüngeren Teilnehmenden (16 - 24 Jahre) in der PIAAC-Studie konnten Solheim und Lundetræ (2017) die Annahme bestätigen, dass sich die Geschlechtsunterschiede in der Lesekompetenz zwischen weiblichen und männlichen Teilnehmenden verändert. Sie stellten in den skandinavischen Ländern für die Kinder in der 4. Klasse kleine Effekte fest $(d=0.19$ - 0.33). Für die 15-Jährigen in PISA zeigten sich hinsichtlich ihrer Stärke mittlere Effekte in Dänemark, Finnland, Norwegen und Schweden $(d=0.34-0.64)$. Hingegen konnten für die jungen Frauen und Männer aus der PIAACStichprobe in den vier untersuchten Ländern keine Geschlechtsunterschiede $(d=0.01-0.12)$ im Lesen festgestellt werden (Solheim \& Lundetræ, 2017).

Zusammenfassend lassen die dargestellten Ergebnisse vermuten, dass sich die Lesekompetenzunterschiede zwischen weiblichen und männlichen Lesenden vom Ende der Grundschule bis in die Sekundarstufe Ivergrößern (Mullis et al., 2017; Solheim \& Lundetræ, 2017; Weis, Doroganova et al., 2019). In den Ergebnissen von PISA zeigten sich bei den 15jährigen Schülerinnen und Schülern die größten Lesekompetenzunterschiede zwischen jungen Männern und Frauen (Weis, Doroganova et al., 2019). Bei De Fraine und Kollegen (2007) konnten differenzielle Entwicklungsverläufe für weibliche und männliche Teilnehmende abgeleitet werden. Die Ergebnisse deuten darauf hin, dass junge Männer in ihren sprachlichen Leistungen, trotz eines Tiefpunktes in der 9. Klasse, am Ende der 12. Klasse zu den Leistungen der jungen Frauen aufholen. Im Erwachsenenalter konnten wiederum keine Geschlechtsunterschiede in der Lesekompetenz nachgewiesen werden (OECD, 2016). Diese Befunde legen folglich die Vermutung nahe, dass sich die geschlechtsspezifischen Unterschiede in der Lesekompetenz im Laufe der Zeit ändern und im Erwachsenenalter abnehmen. 
Die dargestellten Ergebnisse bieten einen ersten Ansatz dahingehend, wie sich die Lesekompetenz von Frauen und Männern über die Zeit, beginnend mit dem Ende der Grundschulzeit bis ins Erwachsenenalter, entwickeln könnte. Allerdings soll an dieser Stelle auf einige Einschränkungen hinsichtlich der Interpretation der Ergebnisse hingewiesen werden. Zum einen beziehen sich einige Daten primär auf Querschnittserhebungen (PIRLS/IGLU, IQB-Bildungstrend, PISA, PIAAC). Zum anderen wurden die meisten Studien in der Sekundarstufe I durchgeführt und nur wenige beziehen sich auf die Sekundarstufe II (Nagy et al., 2017). Keine der aufgeführten Studien kann den Übergang von der Schule in die Zeit danach, und die weitere Kompetenzentwicklung für diese Lebensphase nachzeichnen.

Außerdem sind differierende Studiendesigns und Instrumente zur Messung der Lesekompetenz ein wesentlicher Schwachpunkt. Es ist davon auszugehen, dass jedes verwendete Instrument nach Gütekriterien standardisiert und hinreichenden Qualitätsprüfungen unterzogen worden ist. Allerdings sind die Instrumente untereinander schwer vergleichbar und nicht miteinander verlinkt (z. B. für PIRLS/IGLU und PISA: Artelt, Drechsel, Bos \& Stubbe, 2009; für PISA und PIAAC: OECD, 2013; Solheim \& Lundetræ, 2017). Zwar kann davon ausgegangen werden, dass alle Studien in einer gewissen Form Lesekompetenz messen, jedoch ist anzunehmen, dass es möglicherweise zu Abweichungen in den Messungen kommt.

Auf der Grundlage der genannten Punkte kann das erste Forschungsziel der vorliegenden Synopse formuliert werden. Die Entwicklung der Lesekompetenz von jungen Frauen und Männern soll mit einer Längsschnitt-Messung beschrieben werden. Beginnend mit dem Ende der Pflichtschulzeit in Klasse neun soll geklärt werden, wie sich die Lesekompetenz differenziell für junge Frauen und Männer entwickelt und ob Geschlechtsunterschiede im jungen Erwachsenenalter nach dem Ende der Schulzeit weiterhin vorhanden sind oder ob diese sich nivellieren. 


\section{Determinanten für die Lesekompetenz von Frauen und Männern}

Wie im vorangegangenen Kapitel deskriptiv aufgezeigt, konnten verschiedene Studien signifikante Unterschiede zwischen Mädchen/Frauen und Jungen/Männern in der Lesekompetenz nachweisen. In der weiteren Ausführung werden nun mögliche Erklärungen für Geschlechtsunterschiede in der Lesekompetenz erläutert.

Für eine differenzierte Darstellung der unterschiedlichen Determinanten auf die Lesekompetenz wird auf die Text-Lesenden-Interaktion (Christmann \& Groeben, 1999) und das Tetraedermodell (Artelt et al., 2007) als theoretischer Rahmen zurückgegriffen.

Nach diesen beiden Modellen ist das Lesen von Texten eine aktive Auseinandersetzung mit den gelesenen Inhalten. Die gelesenen Informationen aus dem Text werden dabei aktiv von der Leserin und dem Leser verarbeitet, interpretiert und mit deren Vor- und Weltwissen verknüpft (Christmann \& Groeben, 1999). Beim Verstehen der Inhalte und dem Aufbau eines Situationsmodells spielen zudem die Merkmale eines Textes eine zentrale Rolle. Infolge kommt es zu einer Wechselwirkung zwischen Texten und deren Informationen, Inhalten und Intentionen sowie den Lesenden und deren Wissen. Dies kann als TextLesenden-Interaktion bezeichnet werden (Christmann \& Groeben, 1999; Rost \& Buch, 2018). Darüber hinaus erfolgt die Auseinandersetzung mit Textinhalten und der Austausch über das Gelesene stets in Interaktion mit der sozialen Umwelt (Christmann \& Groeben, 1999; Hurrelmann, 2004; Möller \& Schiefele, 2004).

Über die Text-Lesenden-Interaktion, die soziale Einbettung des Lesens und die gelesenen Inhalte ist es möglich, Quellen für die Varianz in der Lesekompetenz, wie sie beschrieben bei Frauen und Männern vorliegen, genauer zu identifizieren. Denn sowohl auf Seiten der personenbezogenen als auch auf Seiten der textbezogenen Aspekte, sind Determinanten für die Lesekompetenz von Frauen und Männern zu identifizieren. Die soziale Umwelt ist dabei als ein umspannender Rahmen zu sehen, der gleichermaßen einen Einfluss auf die personenspezifischen Determinanten und die textspezifischen Determinanten hat. 
Im Folgenden werden aus dieser beschriebenen Text-Lesenden-Interaktion in Anlehnung an das Tetraedermodell von Artelt und Kolleginnen und Kollegen (2007) verschiedene Aspekte zur Erklärung von Geschlechtsunterschieden in der Lesekompetenz aufgegriffen und genauer betrachtet. Die Darstellung des Tetraedermodells in Abbildung 1 ist an die Darstellung von Lenhard (2019) angelehnt. Für die vorliegende Fragestellung zur Erklärung von Geschlechtsunterschieden in der Lesekompetenz wurde diese eigens angepasst.

Das Tetraedermodell beinhaltet insgesamt vier Merkmalsklassen, welche für das erfolgreiche Lesen und Verstehen von Texten sowie zur Vorhersage der Lesekompetenz von Relevanz sind (Artelt et al., 2007). Diese vier Merkmalsklassen lassen sich wiederum in Determinanten der Lesenden (in Abbildung 1 dunkelgrün umrandet) und in Determinanten der Texte (in Abbildung 1 hellgrün umrandet) kategorisieren.

\section{Lesekompetenz}

als aktiver und interaktiver Prozess über die Lebensspanne hinweg

\section{Soziale Umwelt}

u. a. Geschlecht, Stereotype, Sozialisation, Anschlusskommunikation

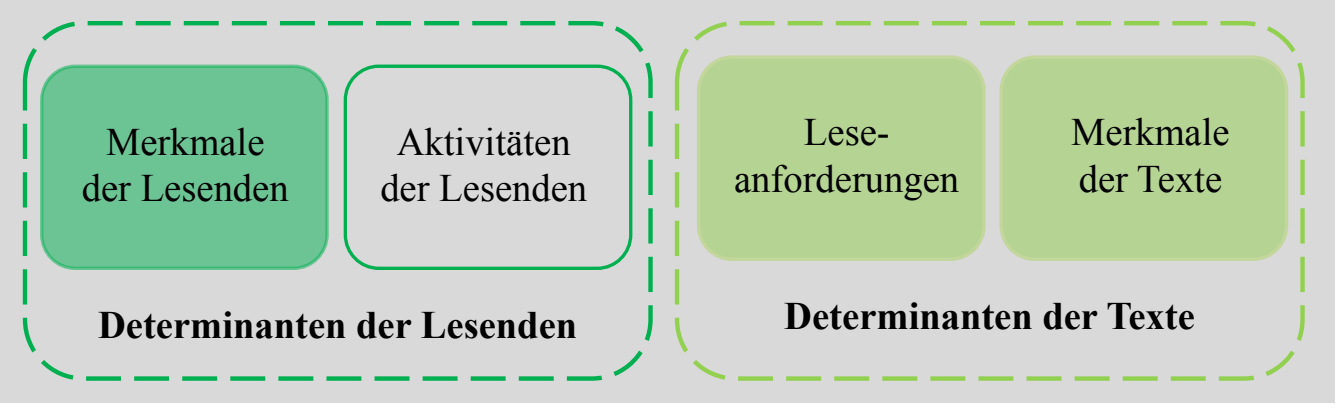

Abbildung 1: Determinanten von Lesekompetenz in Anlehnung an Arteltet al., 2007 und Lenhard, 2019

In dieser Arbeit stehen insbesondere die soziale Umwelt, die verschiedenen Merkmale der Lesenden, die Leseanforderungen und die Merkmale der Texte für die Erklärung von Geschlechtsunterschieden im Vordergrund.

Auf die Aktivitäten der Lesenden (u. a. Lernstrategien, metakognitive Überwachung und Selbstregulation; Artelt et al., 2007) wird nicht näher eingegangen. Hierzu soll an dieser Stelle festgehalten werden, dass neben den motivational-behavioralen Merkmalen (intrinsische und extrinsische Lesemotivation, Lesemenge bzw. -häufigkeit, Lesedauer) auch die kognitive Grundfähigkeit, die Dekodierfähigkeit und das Lernstrategiewissen einen 
entscheidenden Einfluss auf die Lesekompetenz von Personen haben (McElvany \& Becker, 2010; Möller \& Schiefele, 2004). Jedoch sollen die Aktivitäten der Lesenden nicht im Fokus der Ausarbeitung der Fragestellung nach Geschlechtsunterschieden in der Lesekompetenz sowie der anschließenden Ausformulierung der Forschungsfragen stehen. Daher wird diese Determinante in der vorliegenden Arbeit nicht genauer betrachtet. Hingen sollen folgend nun getrennt für einander der Einfluss der sozialen Umwelt, die Merkmale der Lesenden sowie der Texte auf die Lesekompetenz von Frauen und Männern ausgeführt werden, um daraus die Forschungsfragen ausformulieren zu können.

\subsection{Soziale Umwelt}

Im Folgenden wird nun der Einfluss der sozialen Umwelt auf die Lesekompetenz von Frauen und Männern genauer erläutert.

Wie bereits einleitend zu diesem Kapitel aufgeführt, ist das Lesen von Texten und die Auseinandersetzung mit den gelesenen Inhalten eine aktive Tätigkeit. Diese Aktivität ist in einen sozialen Rahmen eingebettet und findet im Austausch mit der Umwelt über das Gelesene seinen Ausdruck (Christmann \& Groeben, 1999). In verschiedenen theoretischen Ansätzen zur Erklärung von Unterschieden in der Lesekompetenz, wird der sozialen Umwelt eine entscheidende Rolle zugeschrieben, da primär Einstellungen, Stereotype und Verhaltensweisen aus dem näheren Umfeld sowie der Gesellschaft weitergegeben werden und in der Folge das individuelle Verhalten und die Motivation beeinflussen (Hurrelmann, 2004; Möller \& Schiefele, 2004; Philipp, 2011).

Im Mehr-Ebenen-Modell der Lesesozialisation nach Hurrelmann (2004), welches sich mit der sozialen Umwelt und deren Einfluss auf die Lesekompetenz befasst, wird der sozialen Umwelt auf der Makro- (Gesellschaft, Kultur) und der Mesoebene (Familie, Schule, Altersgruppe) eine bedeutende Rolle in der Sozialisation der Lesekompetenz zugeschrieben. Über die Makro- und Mesoebene wird das einzelne Individuum in seiner Haltung, Einstellung und seinem Verhalten zum Lesen beeinflusst. Durch die Weitergabe von Bildungsnormen und gesellschaftlichen Einstellungen auf der Makroeben wird wiederum die Mesoebene beeinflusst. Auf der Mesoebene ko-konstruieren schließlich Familien, Lehrkräfte und Freunde diese für sich weiter. Letztendlich wird mittels der verschiedenen 
Prozesse auf der Mikroebene beim einzelnen Individuum eine Vielfalt von einzelnen Handlungsalternativen angestoßen. Es ist möglich, dass die Einstellungen und Normen aus der Mesoebene übernommen werden, aber auch ins Gegenteil umschwenken und zu gegensätzlichem Verhalten führen. So resultiert nach dieser theoretischen Überlegung die Entwicklung der Lesekompetenz aus dem Prozess der Sozialisation auf mehreren sozialen Ebenen (Hurrelmann, 2004; Philipp, 2011).

Eine weitere Theorie, in der die soziale Umwelt ebenso als wichtige Quelle für Unterschiede im Leseverhalten und der Lesekompetenz beschrieben wird, stellt die Erwartungs-WertTheorie nach Eccles und Kolleginnen sowie Kollegen dar (1987; Wigfield, Eccles, Roeser \& Schiefele, 2008). Für die Erklärung der Lesekompetenz wurde dieses Modell von Möller und Schiefele (2004) modifiziert. Im Kern fokussiert die Erwartungs-Wert-Theorie die Vorhersage von Motivation und Verhalten in Leistungssituationen durch eine subjektive Erfolgserwartung („Werde ich die Aufgabe lösen können?“) und eine Wertkomponente („Was ist mir wichtig und macht mir Spaß?“). Der subjektive Wert der Aufgabe und die Erfolgserwartung werden jedoch zentral von der sozialen Umwelt bzw. dem kulturellen Milieu beeinflusst. Ähnlich wie im Mehr-Ebenen-Modell der Lesesozialisation (Hurrelmann, 2004; Philipp, 2011) beinhaltet das kulturelle Milieu der Erwartungs-WertTheorie die schulische und familiäre Umwelt einer Person, das Leseverhalten wichtiger anderer Personen sowie die eigenen Erfahrungen mit dem Lesen. Darüber hinaus werden zur sozialen Umwelt in der Erwartungs-Wert-Theorie auch Geschlechterrollen und lesebezogene Stereotype hinzugenommen (Becker \& McElvany, 2018; Möller \& Schiefele, 2004). Nach Möller und Schiefele (2004) haben vornehmlich sozial vermittelte Geschlechtsstereotype aus der sozialen Umwelt eine besondere Bedeutung. Diese beeinflussen vorranging das individuelle Interesse und, in der Wertkomponente, das Selbstkonzept. Das Ausmaß, in der eine Aufgabe oder Tätigkeit als übereinstimmend mit der eigenen Geschlechterrolle wahrgenommen wird, bestimmt folglich deren Wertigkeit (Möller \& Schiefele, 2004).

Die hier angesprochenen Geschlechtsstereotype definieren sich im Allgemeinen darüber, welche persönlichen Überzeugungen und Erwartungen hinsichtlich Fertigkeiten, Fähigkeiten, Einstellungen, Rollen und Verhaltensweisen Frauen und Männern zugeschrieben werden (Athenstaedt \& Alfermann, 2011; Hannover, 2006). Stereotype sind 
dabei keine Zuschreibungen für einzelne Individuen, sondern gelten als sozialgeteilte Annahmen bezogen auf alle Mitglieder dieser Gruppe. Dabei haben Stereotype zwei Komponenten: Die deskriptive Seite, welche beschreibt, wie Frauen und Männer typischerweise sind, dient der erleichterten Verarbeitung von Informationen aus der sozialen Umwelt. Die andere Komponente von Stereotypen, die präskriptive Seite, drückt wiederum Erwartungshaltungen aus, wie Frauen und Männer typischerweise sein sollten. Diese Standpunkte beruhen auf traditionell definierten Geschlechterrollen und legitimieren gesellschaftlich definierte Unterschiede zwischen Frauen und Männern (Athenstaedt \& Alfermann, 2011; Hannover, 2006).

Hinsichtlich der Aktivität des Lesens lassen sich wiederkehrende Geschlechtsstereotype feststellen. Gelten die Fächer Mathematik, Informatik, Naturwissenschaften und Technik (MINT) als stereotyp männliche Domänen, in denen Jungen/Männer als begabter und interessierter bewertet werden, so gilt dieser Vorteil für Mädchen/Frauen in sprachlichen und künstlerischen Domänen (Heyder \& Kessels, 2013; Steffens \& Jelenec, 2011). Diverse Studien konnten nachweisen, dass Lesen als stereotyp weiblicher Gegenstandsbereich kategorisiert wird und eher Mädchen und Frauen im Gegensatz zu Jungen und Männer als daran interessiert eingeschätzt werden (Nowicki \& Lopata, 2017; Steffens \& Jelenec, 2011; Watson, Kehler \& Martino, 2010). Bereits unter Kindern in der ersten Klasse gibt es stereotype Überzeugungen, was Jungen und Mädchen typischerweise gerne lesen. Diese stereotypen Leseüberzeugungen für andere Kinder entsprechen aber nicht unbedingt den eigenen Präferenzen der Kinder (Chapman, Filipenko, McTavish \& Shapiro, 2007).

Manifestierte Geschlechtsstereotype können in gesprochener und geschriebener Sprache ausgedrückt werden. Garnham, Oakhill, von Stockhausen und Sczesny (2016) führen an, dass die gesprochene und formal verwendete Sprache als kulturspezifische Bedingung eine Möglichkeit darstellt, um die Charakterisierung von Geschlecht und vorhandenen Stereotypen zu spezifisch weiblichen und männlichen Einstellungen, Verhaltensweisen und Rollenzuschreibungen aufrecht zu erhalten bzw. abzubauen. Nach ihrer Argumentation werden geschlechtsbezogene Informationen in der Sprache durch semantische und grammatikalische Kategorien übertragen. Aber auch Rollennamen (z. B. Manager oder Sekretärin), welche nur auf ein Geschlecht bezogen sind und nicht für beide Geschlechter gleichermaßen (z. B. Manager/Managerin oder Sekretärin/Sekretär) verwendet werden, tragen zum Erhalt von Rollenzuschreibungen bei (Garnham et al., 2016). 
In den Studien von McElvany, El-Khechen, Schwabe und Kessels (2016) sowie ElKhechen, McElvany, Wolter und Kessels (2017) konnte herausgestellt werden, dass bereits bei Grundschulkindern ein höherer Wortschatz für stereotyp konnotierte Wörter vorlag, der mit dem eigenen Geschlecht der Kinder übereinstimmte. Auch wenn sich statistisch kein signifikanter Unterschied bei den richtig gelösten Wortschatz-Aufgaben zwischen Jungen und Mädchen zeigte, manifestiert sich bei qualitativer Betrachtung des geschlechtsspezifischen Wortschatzes, dass Jungen und Mädchen jene Wörter häufiger kannten, die mit ihrer eigenen Geschlechtsgruppe assoziiert werden (McElvany et al., 2016). Diese Ergebnisse deuten darauf hin, dass bereits Grundschulkinder stereotype Zuordnungen in ihre eigene Sprache aufnehmen und manifestieren.

Anhand der dargestellten Modelle und Studien wird deutlich, dass die soziale Umwelt bei der Erklärung von Geschlechtsunterschieden im Lesen nicht zu vernachlässigen ist. Besonders stereotype Einstellungen hinsichtlich des Lesens und die Feminisierung dieser Tätigkeit, sind wichtige Faktoren zur Erklärung von Unterschieden im Lesen zwischen Mädchen/Frauen und Jungen/Männern. Ebenso zeigt sich, dass vorhandene Stereotype in Bezug auf Frauen und Männer in gesprochener sowie geschriebener Sprache Ausdruck finden, und den Leser als auch die Leserin dadurch beeinflussen. Diese Einstellungen der sozialen Umwelt können demnach sowohl einen Einfluss auf die Merkmale der Lesenden (in Abbildung 1 dunkelgrün hinterlegt) haben und sich darüber hinaus in den Inhalten sowie der Struktur der Texte (Abbildung 1 hellgrün hinterlegt) widerspiegeln. Im Folgenden sollen diese Determinanten jeweils einzeln betrachtet werden.

\subsection{Merkmale der Lesenden}

Leserinnen oder Leser von Texten sind die wesentlichen Rezipienten der gelesenen Inhalte. Infolgedessen stehen die Lesenden und deren Merkmale für den Leseprozess im Mittelpunkt. $\mathrm{Zu}$ den Merkmalen des Lesers oder der Leserin zählen im Tetraedermodell von Artelt und Kolleginnen sowie Kollegen (2007) das Vorwissen, der Wortschatz, das Wissen über Textmerkmale, die Wortidentifikation, das Lernstrategiewissen sowie die Lesemotivation.

In der weiteren Ausführung wird zur Erklärung von Geschlechtsunterschieden in der Lesekompetenz primär der Fokus auf affektiv-emotionale Konstrukte gelegt. Die 
Lesemotivation ist ein wichtiger Prädiktor für das Leseverhalten (Lesemenge, Ausdauer, Lesehäufigkeit und Präferenzen für verschiedene Textgenres) und hat dadurch vermittelt einen positiven Einfluss auf die Lesekompetenz (McElvany et al., 2008; Miyamoto, Pfost \& Artelt, 2018; Mol \& Bus, 2011; Schiefele, Schaffner, Möller \& Wigfield, 2012). Vielfältige Ergebnisse berichten von deutlichen Unterschieden zwischen Mädchen/Frauen und Jungen/Männern hinsichtlich der Lesemotivation (z. B. Diedrich et al., 2019; McGeown, Duncan, Griffiths \& Stothard, 2015; Philipp, 2015; Schiefele et al., 2012), des Leseverhaltens (Logan \& Johnston, 2009; Pfost, Dörfler \& Artelt, 2013) und den Präferenzen von Textgenres (Charlton, Burbaum \& Sutter, 2004; Schreier \& Odağ, 2004). Diese Differenzen können wiederum bedeutsame Unterschiede in der Lesekompetenz aufklären (z. B. McElvany et al., 2017; Stanat \& Kunter, 2002).

Nach Schiefele (1996) ist die habituelle Lesemotivation einer Person das jeweilige Ausmaß des Wunsches oder der Absicht, in einer bestimmten Situation einen spezifischen Text zu lesen. Dieser Wunsch oder die Absicht einen Text zu lesen, oder auch nicht zu lesen, ist ein sehr facettenreiches und vielschichtiges Konstrukt, welches sich durch verschiedene Motive zusammensetzt (Wigfield \& Guthrie, 1997).

Generell haben sich in der Motivationsforschung zwei zentrale Kategorien zur Bestimmung, warum jemand liest oder nicht liest, durchgesetzt: Die Wünsche oder Absichten des Lesens oder Nicht-Lesens können aus eigenem Antrieb entstehen, da die Tätigkeit des Lesens oder der gelesene Gegenstand bzw. Inhalt als befriedigend oder gewinnbringend angesehen wird (intrinsische Lesemotivation). Bei der extrinsischen Lesemotivation liegen hingegen die Gründe für das Lesen außerhalb des Inhalts oder der Tätigkeit, und werden demnach von den erwarteten, positiven oder negativen Konsequenzen bestimmt (Ryan \& Deci, 2000; Schiefele, 1996; Schiefele et al., 2012).

Forschungsergebnisse zeigen, dass beide Formen der Lesemotivation einen Einfluss auf das Leseverhalten in Form von Lesemenge, Lesehäufigkeit und Präferenzen für verschiedene Textgenres haben und ferner die Lesekompetenz fördern (Artelt et al., 2007; Locher \& Pfost, 2019; McElvany et al., 2017; Mol \& Bus, 2011; Wigfield \& Guthrie, 1997). Pfost, Dörfler und Artelt (2010) nennen diese Reziprozität den „Engelskreis des Lesens“: Eine hohe Lesemotivation führt $\mathrm{zu}$ vermehrtem und ausdauerndem Leseverhalten und hat wiederum einen positiven Einfluss auf die Lesekompetenz. 
Die Forschungsarbeiten um die Gruppe von Guthrie und Wigfield postulieren außerdem, dass ein gesteigertes Leseverhalten und regelmäßiges Lesen den Leseprozess auf den verschiedenen hierarchischen Teilprozessen auf Wort-, Satz- und Textebene (siehe Kapitel 2) nachhaltig beeinflussen. Auf der hierarchieniedrigeren Ebene regt das häufige Lesen die Automatisierung von Prozessen an und hat eine positive Wirkung hinsichtlich der Effizienz der Dekodierung und des Einsatzes von Strategien. Überdies wird der Wortschatz um neue Wörter, und das Vorwissen durch die neuen Informationen und Inhalte erweitert und mit bereits vorhandenem Wissen verknüpft, was die vorhandene Kohärenz sowie das Situationsmodell ausbaut. Außerdem wird davon ausgegangen, dass das Leseselbstkonzept durch das Leseverhalten unterstützt wird und sich der Leser oder die Leserin dadurch schwierigeren Texten widmet (Guthrie, Wigfield, Metsala \& Cox, 1999; Guthrie \& Wigfield, 2009). Entsprechend des „Engelskreises des Lesens“ existiert gleichermaßen der sogenannte „Teufelskreis des Nicht-Lesens“. Dieser entspricht einer geringen Lesemotivation, fehlender Lektüre und daraus resultierend Defiziten in der Lesekompetenz (Pfost et al., 2010).

Die differenzierte Darstellung des (motivationalen) Leseverhaltens verdeutlicht einen essentiellen Einfluss auf die Lesekompetenz und deren Entwicklung. Das Leseverhalten kann durch verschiedene Möglichkeiten operationalisiert werden. Schiefele und Kollegin sowie Kollegen (2012) zählen zum Leseverhalten die Lesemenge, der Einsatz von Strategien und die individuellen Präferenzen von verschiedenen Textgenres. Nach Locher und Pfost (2018) lässt sich das Leseverhalten durch ein Globalurteil des Lesevolumens quantifizieren (Wie viel wird gelesen?) und durch ein differenziertes textsortenspezifisches Urteil qualifizieren (Was wird gelesen?). Durch die Analyse dieser beiden Aspekte wie viel und im Speziellen was von Personen gelesen wird, können das Interesse und die Einstellungen zum Lesen gefördert, und somit eine positive Auswirkung auf das Lesen erwirkt werden (McGeown, 2015).

Groeben (2004) nimmt in seiner theoretischen Ausarbeitung eine qualifizierenden Einteilung des individuelle Leseverhaltens vor. Er beschreibt mit den „Funktionen des Lesens" die leser- und leserinnenseitige Intention warum sowie zu welchem Zweck eine Person liest und mit welcher Präferenz sie oder er sich für einen bestimmten Textinhalt entscheidet. Er unterteilt dabei den Lesezweck einerseits in fiktionales Lesen von narrativen Texten zur Unterhaltung, dem ästhetischen Genuss, der Spannung und dem Fantasieren 
sowie andererseits in non-fiktionales Lesen von expositorischen Texten zur Informationsaufnahme, Bewertung, Handlungskompetenz, zum Wissenserwerb und Verständnis von Sachverhalten. Mit dem Prozess des Lesens korrelieren beim Leser oder der Leserin verschiedene Intentionen. Das Lesen von fiktionalen Texten zur Unterhaltung soll primär der Fantasieentwicklung dienen, aber auch Empathie, Moralbewusstsein und Anerkennung von Andersartigkeit stärken. Hingegen soll das Lesen von non-fiktionalen Texten zur Information beim Leser oder der Leserin die argumentative Kommunikation anregen und zu einer Meinungsbildung sowie Wissensvertiefung beitragen (Groeben, 2004). Die Unterscheidung nach Lesepräferenzen ist dabei nicht als dichotome Gegenüberstellung zu verstehen. Vielmehr stellt es ein Kontinuum dar, bei dem ebenso gemischte Formen von Lesepräferenzen vorhanden sind. Des Weiteren ist das Textmaterial nicht immer eindeutig zu klassifizieren, vielmehr sind Mischformen möglich (Groeben, 2004).

Besonders im Freizeitlesen haben die Leserinnen und Leser eine große Bandbreite aus möglichen Genres, Themengebieten und Darstellungsformen (digital oder gedruckt) zur Verfügung, aus denen sie nach ihren eigenen Präferenzen, Interessen und Gewohnheiten wählen können. In der Meta-Analyse von Mol und Bus (2011) konnte für das Freizeitleseverhalten, gemessen durch Print Exposure (operationalisiert über Autorinnenbzw. Autorennamen sowie Buchtitel) als Proxy für die Lesemenge, und dessen Einfluss auf das Leseverständnis, den Wortschatz, die basalen Lesefertigkeiten und die Rechtschreibung moderate bis starke Zusammenhänge gefunden werden. Insgesamt konnte in dieser Analyse für alle untersuchten Altersgruppen (Kindergarten, Schülerinnen und Schüler in den Klassen 1-2, Studierende) durch ein gesteigertes Freizeitleseverhalten eine Zunahme in der Lesefähigkeit festgestellt werden. Jedoch war der Effekt der Buchexposition auf das Leseverstehen bei den Studierenden $(M=21$ Jahre, $S D=2.32)$ mit $d=0.45 \mathrm{im}$ Vergleich zu den anderen beiden Altersgruppen am stärksten ausgeprägt. Die Autorinnen führen dies darauf zurück, dass die erwachsenen Leserinnen und Leser im Vergleich zu den Kindern und Jugendlichen ihre Freizeitgestaltungen selbstbestimmter aussuchen und damit die Auswahl des Inhalts des Lesestoffes autonomer erfolgt (Mol \& Bus, 2011).

Wie bereits verdeutlicht, zeigen sich hinsichtlich vorhandener Geschlechtsunterschiede in der Lesekompetenz in zahlreichen Studien, dass der Unterschied zwischen Mädchen/Frauen und Jungen/Männern durch deren unterschiedliche Lesemotivation (z. B. Becker et al., 2010; Schiefele et al., 2012; Schwabe, McElvany \& Trendtel, 2015; Stanat \& Kunter, 2002), die 
Einstellung zum Lesen(Logan \& Johnston, 2009) und der Lesemenge (Schaffner, Philipp \& Schiefele, 2016) erklärt werden kann. Verbunden mit dem oben beschriebenen Stereotyp, dass Lesen als weibliche Tätigkeit gilt und Mädchen/Frauen im Lesen als begabter und interessierter eingeschätzt werden (Heyder \& Kessels, 2013; Steffens \& Jelenec, 2011; Watson et al., 2010), drückt sich dieses Stereotyp dadurch aus, dass Mädchen im Allgemeinen eine höhere intrinsische Lesemotivation haben (z. B. Becker et al., 2010; Schiefele et al., 2012; Schwabe et al., 2015; Stanat \& Kunter, 2002) und in ihrer Freizeit im Durchschnitt mehr lesen als Jungen (McGeown, Goodwin, Henderson \& Wright, 2012; Schiefele et al., 2012). In den PISA-Daten von 2018 lässt sich diesbezüglich feststellen, dass sich im Durchschnitt mehr junge Männer als Frauen unter den 15-Jährigen in der Gruppe der „Lesemuffel“" befinden. Dies wird unter anderem dadurch deutlich, dass $61 \%$ der befragten jungen Männer angaben, nicht zu ihrem Vergnügen zu lesen. Des Weiteren haben junge Frauen mit einem Effekt von $d=0.70$ eine höhere Freude am Lesen (operationalisiert über die Items: „Lesen ist mein Hobby“; „Ich rede gerne mit anderen Leuten über Bücher“; „Lesen ist keine Zeitverschwendung“; Diedrich et al., 2019).

Nicht nur hinsichtlich der angeführten intrinsischen Motivation und des Lesevolumens lassen sich Unterschiede zwischen Frauen und Männern feststellen. Es zeigen sich ebenfalls unterschiedliche Präferenzen in der Auswahl der Themen und in den Motiven, warum Frauen und Männer bestimmte Inhalte lesen.

Philipp (2011) leitete in einer Übersicht aus mehreren Studien ab, dass Lesepräferenzen von Kindern und Jugendlichen zwischen den Klassenstufen 2-10 sehr vielfältig sind und sowohl fiktionale Texte als auch Sachtexte gelesen werden, wobei geschlechtsspezifische Unterschiede bei den Inhalten erkennbar sind. So präferieren Jungen im Allgemeinen eher Themen, bei denen es um Sport, Technik und „gruselige“ Abenteuer geht. Mädchen hingegen lesen lieber Inhalte mit Alltagsbezug, über gleichaltrige Personen oder Idole bzw. Vorbilder (Philipp, 2011).

Die Stiftung Lesen (2008) konnte hinsichtlich des Anlasses zum Lesen in einer Umfrage unter 2,552 Deutschen ab 14 Jahren deskriptiv feststellen, dass Frauen im Vergleich zu Männern häufiger Bücher zur Unterhaltung lesen (37 \% Frauen gegenüber 20 \% Männer), Männer hingegen häufiger realistische Fakten als Frauen bevorzugen (51 \% Männer gegenüber $42 \%$ Frauen). Im Vergleich zu Männern wollen Frauen beim Lesen häufiger den Alltag vergessen (52\% gegenüber $33 \%$ ), sich in andere Charaktere 
einfühlen (31\% gegenüber $19 \%)$ und durch das Lesen in eine andere Welt entfliehen (28\% gegenüber $17 \%$ ).

In einer Interviewstudie mit 1,025 Personen aus Deutschland zwischen 16 und 88 Jahren, durchgeführt von Charlton, Burbaum und Sutter (2004), wurde die emotionale Auseinandersetzung mit literarischen Texten erfasst. Die Ergebnisse der Studie legen nahe, dass Frauen sich im Allgemeinen mehr mit den Charakteren identifizieren und die gelesenen Inhalte stärker in ihren Alltag übertragen als Männer. In einer weiteren Analyse der selben Datengrundlage stellten Schreier und Odağ (2004) fest, dass Frauen stärker in fiktive Texte involviert sind, wohingegen Männer stärker zwischen Fiktion und Alltag abstrahieren können.

Zusammenfassend zeigen die Ergebnisse, dass beim Lesen von Texten nicht ausschließlich kognitive Verstehensleistungen und das Extrahieren von Informationen von Relevanz sind. Für ein umfassendes reziprokes Lesekompetenz-Konzept müssen die motivationalbehavioralen Einflussfaktoren ebenfalls beachtet werden (Christmann \& Groeben, 1999; McElvany \& Becker, 2010; Weinert, 2001).

Dabei lassen sich bezüglich der Lesefreude, der Lesemenge und dem Vergnügen am Lesen Geschlechtsunterschiede zwischen Frauen und Männern feststellen. Im Allgemeinen haben Mädchen/Frauen eine positivere Einstellung zum Lesen und lesen häufiger (u. a. Logan \& Johnston, McGeown et al., 2012; 2009; Schaffner et al., 2016). Ferner deuten die Ergebnisse auf unterschiedliche Präferenzen hinsichtlich der Inhalte zwischen Frauen und Männern hin (Charlton et al., 2004; Schreier \& Odağ, 2004). Obwohl viele Überschneidungen in der Lesepräferenz zu beobachten und diese nicht als Entweder-oderKategorie $\mathrm{zu}$ verstehen sind, lesen Frauen insgesamt in ihrer Freizeit häufiger zur Unterhaltung, um dem Alltag zu entfliehen und sich mit Charakteren aus dem Gelesenen zu identifizieren. Männer hingegen entwickeln durchschnittlich eher Präferenzen für Inhalte, die sie informieren und durch die sie Wissen generieren können.

An dieser Stelle ist darauf hinzuweisen, dass viele der erwähnten Ergebnisse zum Leseverhalten bei Kindern und Jugendlichen erhoben wurden (z. B. Becker \& McElvany, 2018; Diedrich et al., 2019; Logan \& Johnston, 2009; McGeown et al., 2015; Miyamoto et al., 2018; Pfost \& Artelt, 2013; Stanat \& Kunter, 2002). Infolgedessen ist wenig darüber bekannt, wie sich das qualitative Freizeitleseverhalten von Erwachsenen gestaltet (z. B. 
Charlton et al., 2004; Schreier \& Odağ, 2004) und ob ein ähnlich positiver Einfluss auf die Lesekompetenz von Frauen und Männern vorliegt.

\subsection{Merkmale der Texte}

Nach der Darstellung der Umwelt- und leserbezogenen Determinanten liegt in diesem Kapitel der Schwerpunkt auf den textbezogenen Determinanten der Lesekompetenz. Hierbei ist essentiell, dass der Leser oder die Leserin zwar die zentralen Rezipienten eines Textes sind, sich der Inhalt und die Botschaft für die Lesenden jedoch durch den geschriebenen Text entwickeln. Anhand der Leseanforderungen sowie diverser Merkmale der Texte, wird es der Leserin oder dem Leser erleichtert bzw. erschwert, ein Situationsmodell aufzubauen (Artelt et al., 2007).

Im Folgenden sollen die verschiedenen Aspekte der Determinanten der Texte genauer erläutert werden. Im Anschluss daran werden bisherige Befunde zu den Textmerkmalen und deren Einfluss auf die Lesekompetenz von Frauen und Männern dargestellt.

Der jeweilige Zweck des Lesens oder die Lesepräferenz für einen bestimmten Inhalt findet seinen Ausdruck in der entsprechenden Wahl der Textgattung. Das Zusammenspiel von Auswahl des Lesestoffes für die jeweilige Leseabsicht oder Lesepräferenz spiegelt die TextLesenden-Interaktion wider. Ein Leser oder eine Leserin wird sich demnach für oder gegen einen bestimmten Inhalt entscheiden, weil sie oder er eine bestimmte Absicht mit dem Lesen eines Textes verfolgt. Artelt und Kolleginnen sowie Kollegen (2007) führen vier idealtypische Inhaltsgebiete auf, wie sich der Zweck des Lesens in den Merkmalen von Textgattungen widerspiegelt:

a) Verstehendes Lesen: Durch eine sinnorientierte Informationsverarbeitung können die Informationen aus dem Text mit dem Vorwissen der Lesenden verknüpft werden, um daraus möglichst nahe am Text Schlussfolgerungen zu ziehen und folglich ein Situationsmodell aufzubauen. Beim verstehenden Lesen kommen vor allem kognitive und metakognitive Strategien zum Tragen (Artelt et al., 2007). 
b) Kritisches Lesen: Beim kritischen Lesen sollen vor allem die im Text enthaltenen Inhalte hinsichtlich ihrer Begriffsbedeutungen, ihrer logischen Strukturen und Argumente sowie ihrer inhaltlichen Gültigkeit kritisch hinterfragt werden. Diese aktive Analyse des Inhalts soll für den Leser oder die Leserin die Bewertung der Intention des Textes sowie die inhaltliche Schlussfolgerung erleichtern (Artelt et al., 2007).

c) Reflexives Lesen: Mit dem reflexiven Lesen sollen tiefgehende Verarbeitungsprozes se angeregt werden, welche das Überdenken und Hinterfragen der Textinhalte anstößt. Nicht nur die Inhalte der Texte sollen beim Lesen überdacht werden, sondern gleichermaßen das eigene Denken und Handeln um daraus möglicherweise eine Perspektivänderung und Weiterentwicklung der eigenen Persönlichkeit zu initiieren (Artelt et al., 2007).

d) Involviertes Lesen: Beim involvierten Lesen tauchen die Leserin oder der Leser ins Gelesene sowohl kognitiv als auch emotional ein (Artelt et al., 2007).

Die aufgeführten Leseanforderungen finden ihren Ausdruck in Textgattungen, welche sich prototypisch in die Funktion von Lesen zur Information durch Sachtexte und in die Funktion von Lesen zur Unterhaltung bzw. der ästhetischen Bildung durch literarische Texte kategorisieren lassen (Rosebrock, 2016).

Christmann und Groeben (2009) nehmen eine weitere Systematisierung der vielfältigen Sachtexte in Lehr-, Persuasions- bzw. argumentierende Texte und Instruktionstexte vor. Jede Textgattung der drei Unterkategorien der Sachtexte setzt spezifische Anforderungen an den Leser oder die Leserin, welche sehr ähnlich den vier oben aufgelisteten Kriterien sind. Sachtexte sollen primär Informationen und Erkenntnisse aus dem Alltag vermitteln und weitergeben. Dies würde mit dem verstehenden und kritischen Lesen übereinstimmen. Sachtexte sind sprachlich präzise im Ausdruck, mit dem Anspruch der klaren Verständlichkeit formuliert (Gehrer \& Artelt, 2013; Rosebrock, 2016).

Literarische Texte haben hingegen die Funktion, Welten zu erschaffen sowie Realität und Fiktion verschwimmen zu lassen (Christmann \& Groeben, 2009). In Abgrenzung zu den Sachtexten sind die Deutung und Interpretation von literarischen Texten nicht immer eindeutig herauszulesen. In (modernen) literarischen Texten wird nur in Teilen alles 
sprachlich explizit ausgedrückt. Die Lesenden sollen sich vielmehr selbst emotional und gedanklich in einen Text involvieren können. Dies spiegelt sich in der verwendeten sprachlichen und inhaltlichen Ausgestaltung von Texten wider. Häufiger als in Sachtexten werden in literarischen Texten Mehrdeutigkeiten, explizite und implizite Andeutungen, Verweise und Rückblenden in der Sprache und dem Inhalt erschaffen. Außerdem wird in literarischen Texten bewusster mit (verfremdenden) Stilmitteln, Metaphern und Montagetechniken gearbeitet, um sich syntaktisch und semantisch von der formellen sowie informierenden Sprache von Sachtexten abzugrenzen. Gleichzeitig kann durch diese inhaltlichen und sprachlichen Besonderheiten der literarischen Texte auch deren Verständlichkeit und Interpretation für den Leser und die Leserin erschwert werden, was literarische Texte als komplexere Textsorten im Vergleich zu Sachtexten erscheinen lassen (Gehrer \& Artelt, 2013; Rosebrock, 2016).

Wie bereits bei den Lesepräferenzen aufgeführt (siehe Kapitel 4.2), ist ebenso das Textmaterial nur stellenweise eindeutig zu kategorisieren. Ein literarischer Text hat gleichermaßen den Anspruch kritisches Lesen anzuregen und beinhaltet ferner bestimmte Informationen für die Leserin oder den Leser. Daher ist eine eindeutige Zuordnung nicht immer möglich und vielleicht auch nicht immer intendiert (Groeben, 2004).

Neben den Leseanforderungen des jeweiligen Lesestoffes sind weitere Merkmale von Texten zu identifizieren, welche durch die Organisation und Strukturierung von Inhalten zum Verstehen von Texten entscheidend beitragen.

So ermöglicht eine kohärente Inhaltsorganisation eines Textes dem Leser oder der Leserin ein leichtes Verständnis für den Aufbau und die Interdependenz von Sätzen und Textinhalten. Bei der Inhaltsorganisation werden dabei die lokale (Verknüpfung aufeinanderfolgender Sätze) und globale Kohärenzebene (übergeordnete Textthemen, Verknüpfungen darüber hinaus) eines Textes unterschieden. Fehlen die lokalen und globalen Zusammenhänge in einem Text, so entstehen Lücken und der Leser oder die Leserin muss für das Verständnis zeitaufwändig nach Bezügen suchen (Artelt et al., 2007; van Dijk \& Kintsch, 1983).

Daher ist es für die Leserin oder den Leser des Textes umso einfacher, Kohärenz herzustellen, je klarer und strukturierter die Abfolge von Textinformationen ist. Häufige thematische Sprünge innerhalb eines Textes führen dazu, dass der Leser oder die Leserin den Inhalt schwieriger nachvollziehen kann (Artelt et al., 2007; Schnotz, 2006). 
Sehr hilfreich für das Verstehen von Textinhalten und für die Kohärenzbildung ist die Verknüpfung des gelesenen Inhaltes mit dem eigenen Vorwissen. Insbesondere Analogien oder Metaphern in Texten sind geeignete stilistische Möglichkeiten, um Vorwissen zu aktivieren und auf neue Textinhalte zu beziehen (Artelt et al., 2007).

Mehrere Studien konnten feststellen, dass Geschlechtsunterschiede im Lesen stark nach den eingesetzten Textsorten variieren und dadurch Unterschiede zwischen Mädchen/Frauen und Jungen/Männern erklärt werden können. So ist der Einsatz von kontinuierlichen vs. nicht kontinuierlichen Texten und narrativen Texten vs. Informationstexten ein möglicher Faktor um Geschlechtsunterschiede in der Lesekompetenz zu erklären (Lafontaine \& Monseur, 2009; Solheim \& Lundetræ, 2017).

In der Studie von Stanat und Kunter (2002) wurde die Lesekompetenz der jungen Männer und jungen Frauen in der internationalen PISA-Stichprobe 2000 in Abhängigkeit der verwendeten Textformate genauer betrachtet. Die Ergebnisse deuten darauf hin, dass die 15jährigen Frauen besonders bei kontinuierlichen Texten, in denen die Informationen des Textes fortlaufend dargestellt werden, wie zum Beispiel in Erzählungen oder Argumentationen, eine höhere Lesekompetenz im Vergleich zu den jungen Männern erzielen. Bei nicht-kontinuierlichen Texten, wie zum Beispiel Grafiken, Tabellen oder Diagrammen, sind die Unterschiede in der Lesekompetenz zwischen weiblichen und männlichen Teilnehmenden deutlich kleiner.

Interessanterweise zeigte die Studie, dass sich der Vorteil der jungen Frauen bei den kontinuierlichen Texten reduziert, wenn das Leseinteresse („Lesen macht mir Spaß“; „Ich lese in meiner Freizeit") als Mediator beachtet wurde. Bei den nicht-kontinuierlichen Texten zeigte sich dadurch sogar ein kleiner Vorteil für die jungen Männer. Die Autorinnen vermuten bei diesem Effekt, dass jungen Männern das Format der nicht-kontinuierlichen Texte näher liegt, da dies ihre Interessen und Erfahrungen aus dem mathematischnaturwissenschaftlichen Bereich aufgreift (Stanat \& Kunter, 2002).

Aufbauend auf dem dargestellten Ergebnis von Stanat und Kunter (2002) gehen Artelt und Schlagmüller (2004) in ihren Analysen der Fragestellung nach, ob der Vorteil der jungen Frauen in der Lesekompetenz auf das Format der Texte (kontinuierliche vs. nichtkontinuierliche Texte) oder auf deren Inhalte (literarische Texte bestehend aus Romanauszügen, einer Fabel und einem Drama) zurückzuführen ist. Sie argumentieren, wie im Vorfeld angesprochen, dass der kompetente Umgang mit literarischen Texten ein 
spezifischer Aspekt der Lesekompetenz ist, da bei literarischen Texten unterschiedliche rhetorische Anforderungen und Stilmittel, wie Metaphern oder Symbole, verwendet werden. In der Analyse der internationalen PISA-Daten konnte hierzu festgestellt werden, dass ein Vorteil der jungen Frauen sowohl bei den kontinuierlichen als auch bei den literarischen Texten vorlag. Gemittelt über alle teilnehmenden Länder, konnte aber kein deutlich stärkerer Effekt für die literarischen Texte beobachtet werden. Somit finden sich die ermittelten Lesekompetenzvorteile der jungen Frauen in fortlaufend geschriebenen Texten etwa in der gleichen Größe sowohl für das Format wie auch für den Inhalt von Texten (Artelt \& Schlagmüller, 2004).

Neben den Textmerkmalen wurde wiederkehrend in den Item-Formaten von Lesekompetenztests eine mögliche Quelle der Varianz für die Stärken und Schwächen von Jungen/Männern und Mädchen/Frauen festgestellt. Diese sollen hier auch eine Erwähnung finden.

Mögliche Aufgabenformate für die Messung der Lesekompetenz, welche häufig zum Einsatz kommen, sind geschlossene Antwortformate zum Beispiel durch Multiple-Choice (MC) Aufgaben oder ein offenes Antwortformat. Die Vorteile bei der Verwendung von MCAufgaben in Tests ist die Effizienz in der Durchführung und beim Scoring. Gleichzeitig sprechen jedoch MC-Aufgaben und ein offenes Antwortformat unterschiedliche sprachliche Ebenen an. Beim offenen Antwortformat sind vermehrt rezeptive und produktive Sprachkenntnisse nötig, wobei dieses Format gleichzeitig eine höhere Bearbeitungsmotivation erfordert, um in ausführlicheren Sätzen zu antworten, was wiederum eines höheren Zeitaufwandes bedarf (Haladyna \& Rodriguez, 2013). In den Studien von Lafontaine und Monseur (2009) sowie Schwabe, McElvany und Trendtel (2015) konnte mit Daten aus PISA-Erhebungen gezeigt werden, dass vor allem bei einem offenen Antwortformat die teilnehmenden jungen Frauen einen Vorteil haben. Unter Kontrolle der Leseleistung gaben ebenso die jungen Frauen bei offenen Antworten häufiger eine korrekte Antwort als die jungen Männer. Die Studie von Schwabe und Kollegin sowie Kollege (2015) machte außerdem deutlich, dass sich dieser Effekt gleichfalls unter Kontrolle der intrinsischen Lesemotivation, wie sie in PISA 2009 gemessen wurde, nicht verringerte. Allerdings konnte bei den 15-jährigen jungen Frauen eine höhere intrinsische Lesemotivation nicht auf ihren Vorteil in den offenen Antworten zurückgeführt werden. Es wird vermutet, dass andere Aspekte, wie zum Beispiel die Textsorten (narrative vs. 
expositorische Texte), welche in der Studie nicht berücksichtigt werden, einen größeren Einfluss auf das Antwortformat haben als die intrinsische Lesemotivation (Schwabe et al., 2015).

Hinsichtlich der Text-Lesenden-Interaktion kann somit festgehalten werden, dass die Darstellung und Aufbereitung des Textes einen Einfluss auf das Verstehen eines Textes nehmen. Je strukturierter und kohärenter ein Text aufgebaut ist, desto einfacher ist es für den Leser oder die Leserin eine mentale Repräsentation herzustellen.

Gleichzeitig wurde die enge Korrelation zwischen den Lesepräferenzen (beschrieben in Kapitel 4.2) und dem Textinhalt in Form der Leseanforderungen bestimmter Textgattungen herausgearbeitet. Demnach wird ein Leser oder eine Leserin nach ihren oder seinen jeweiligen Präferenzen den entsprechenden Text mit den jeweiligen Anforderungen wählen.

Die bisherige Forschung zur Erklärung von Geschlechtsunterschieden in der Lesekompetenz lässt vermuten, dass das verwendete Textmaterial in Lese-Assessments von Relevanz ist. Besonders bei kontinuierlichen, wie auch bei literarischen Texten scheinen im Allgemeinen junge Frauen einen Vorteil zu haben (Artelt \& Schlagmüller, 2004).

Wie bereits beim Vergleich der Ergebnisse in Kapitel 3 erwähnt, ist es indes schwierig verschiedene Assessments untereinander $\mathrm{zu}$ vergleichen. Im detaillierten Vergleich der Studien PIRLS 2011, PISA 2009 und PIAAC 2012 von Solheim und Lundetræ (2017) wurde insbesondere in einer Gegenüberstellung verdeutlicht, an welchen Stellen spezifische Unterschiede der Assessments liegen. So wurden zum Beispiel in der PIAAC-Studie nur Sachtexte und keine narrativen Texte zur Messung der Lesekompetenz bei Erwachsenen eingesetzt. Die Autorinnen resümieren, dass das Studiendesign von PIAAC eher den Stärken des Lesens der Männer entspricht und infolge mögliche Geschlechtsunterschiede im Lesen verdeckt. Wiederum ist das Design von PISA eher der Lesekompetenz der jungen Frauen zuträglich und entspricht weniger den Stärken der jungen Männer im Lesen (Solheim \& Lundetræ, 2017).

Daher ist für die Erklärung von Geschlechtsunterschieden in der Lesekompetenz eine differenzierte Betrachtung des verwendeten Materials in einem Lesekompetenztest ebenso wichtig, wie die Determinanten der Lesenden in eine Untersuchung einzubeziehen. 


\section{Problemstellungen und Forschungsfragen der vorliegenden Arbeit}

Abgeleitet aus dem vorangegangenen Forschungsstand, sollen nun im Folgenden die Forschungslücken erläutert und daraus die Forschungsfragen für die vorliegende Arbeit induziert werden.

\subsection{Forschungsfrage 1: Lesekompetenzunterschiede bei jungen Erwachsenen}

In der Arbeit wurde vielfach aufgezeigt, dass Geschlechtsunterschiede in der Lesekompetenz bei Kindern und Jugendlichen vorliegen: Jungen weisen im Kindes- und Jugendalter im Allgemeinen niedrigere Lesekompetenzwerte auf als Mädchen (z. B. Berendes et al., 2018; McElvany et al., 2017; Weis, Doroganova et al., 2019). Im Erwachsenenalter zeigen sich allerdings keine signifikanten Lesekompetenzunterschiede zwischen Frauen und Männern (OECD, 2016). Besonders bei den jüngeren Teilnehmenden in der PIAAC-Studie (zwischen 16 - 24 Jahren) zeigen sich keine Geschlechtsunterschiede in der Lesekompetenz (Maehler et al., 2013; OECD, 2016).

Der Überblick zu den bisherigen Ergebnissen der Lesekompetenzunterschiede zwischen Mädchen/jungen Frauen und Jungen/jungen Männern lässt infolge vermuten, dass die Geschlechtsunterschiede zwischen dem zehnten und fünfzehnten Lebensalter zunehmen (z. B. Berendes et al., 2018; McElvany et al., 2017; OECD 2019; Solheim \& Lundetræ, 2017; Weis, Doroganova et al., 2019). Dann jedoch nehmen im weiteren Lebensverlauf zwischen 16 Jahren und dem Erwachsenenalter die Geschlechtsunterschiede ab und sind schlussendlich nicht mehr vorhanden (Maehler et al., 2013; OECD, 2016).

Für eine mögliche Erklärung der Kompensation des Geschlechtsunterschiedes bei jungen Erwachsenen im Lesen kann abermals die positive Verbindung zur Lesemotivation und dem Leseverhalten herangezogen werden (McElvany et al., 2008; Miyamoto et al., 2018; Mol \& Bus, 2011; Schiefele et al., 2012; Stanat \& Kunter, 2002). Möglicherweise könnte es mit voranschreitendem Alter und dem Verlassen des institutionellen Lernkontextes zu einer besseren Passung der eigenen Ziele und Fähigkeiten kommen, welche mit vermehrt selbstgewählten Leseanlässen und -möglichkeiten der jungen Erwachsenen einhergeht. So 
könnten sich häufiger selbst gewählte Lesepräferenzen wiederum positiv auf das Leseverhalten und die Lesemenge auswirken, und so zu einem Anstieg der Lesekompetenz führen.

Wie im vorangegangenen Überblick zum aktuellen Forschungsstand bereits angemerkt wurde, wurde bei den bisherigen Längsschnitt-Studien zu Lesekompetenzunterschieden überwiegend die Kompetenz von Schülerinnen und Schülern in den Fokus genommen (z. B. Berendes et al., 2018; De Fraine et al., 2007; Nagy et al., 2017; Weis, Doroganova et al., 2019). Infolgedessen fehlen Längsschnitt-Ergebnisse zu Geschlechtsunterschieden in der Lesekompetenzentwicklung nach der Sekundarschulzeit, um detaillierter Aussagen über die weitere Entwicklung der Lesekompetenz von jungen Frauen und Männern machen zu können.

Im Allgemeinen ist bei der Gesamtbetrachtung der dargelegten Befunde darauf hinzuweisen, dass die vorhandenen Ergebnisse der verschiedenen dargestellten Studien zur Lesekompetenzentwicklung nicht auf einer gemeinsamen Längsschnitt-Studie basieren. Da zudem in den Studien differierende Stichproben und Instrumente zur Lesekompetenzmessung herangezogen wurden, ist auch die Vergleichbarkeit der Ergebnisse nur eingeschränkt möglich (z. B. Lundetræ, Sulkunen, Gabrielsen \& Malin, 2014).

Auf Grundlage dieser Forschungslücke kann die erste Forschungsfrage für die vorliegende Arbeit formuliert werden.

Wie eingangs angeführt, ist ein Ziel der Forschungsarbeit, Geschlechtsunterschiede in der Lesekompetenz von jungen Frauen und Männern im Längsschnitt zu beschreiben. Die Lesekompetenz wird hierfür über die Schulzeit hinausgehend bis ins junge Erwachsenenalter betrachtet, da vor allem eine Lücke in der Forschung zur Entwicklung der Lesekompetenz von jungen Frauen und Männern nach dem Verlassen des institutionellen Kontextes der Schule besteht. Beginn wird hierfür die neunte Klasse bzw. das Ende der Pflichtschulzeit sein. Es soll geklärt werden, ob Lesekompetenzunterschiede bei jungen Frauen und Männern weiterhin vorhanden sind oder, ob diese sich verringern bzw. ganz verschwinden. Diese Fragestellung wird demgemäß im Längsschnitt mit einer Stichprobe beginnend in der neunten Klasse (ca. 16 Jahre) über die Schulzeit hinaus beantwortet. Außerdem wird die Lesekompetenz der jungen Frauen und Männer mit einem einheitlichen und verlinkten Instrument gemessen. 
Die erste Forschungsfrage lautet somit:

Wie entwickeln sich die Geschlechtsunterschiede in der Lesekompetenz bei jungen Frauen und Männern zwischen 16 bis 22 Jahren?

\subsection{Forschungsfrage 2: Merkmal auf Seiten der Lesenden}

Auf Seiten der Lesenden konnte vor allem in Kapitel 4.2 herausgearbeitet werden, dass die Lesemotivation einen positiven Einfluss auf das Leseverhalten hat und dies wiederum die Lesekompetenz fördert (z. B. Mol \& Bus, 2011; Pfost et al., 2010; Schiefele et al., 2012; Wigfield \& Guthrie, 1997). Besonders zur Erklärung der Geschlechtsunterschiede in der Lesekompetenz erscheint die Verbindung der Lesekompetenz und den motivationalbehavioralen Einstellungen zum Lesen einen wichtigen Zusammenhang darzustellen (z. B. Becker et al., 2010; McGeown et al., 2012; Schiefele et al., 2012; Stanat \& Kunter, 2002).

Im Allgemeinen kann weitgehend konstatiert werden, dass die Lesemotivation von Mädchen im Durchschnitt höher ist als die der Jungen (z. B. Diedrich et al., 2019; McGeown et al., 2015; Philipp, 2015; Schiefele et al., 2012). Daneben sind im Leseverhalten (Logan \& Johnston, 2009; Mol \& Bus, 2011; Pfost et al., 2013) und bei den Präferenzen von Textgenres (Charlton et al., 2004; Schreier \& Odağ, 2004) Unterschiede zwischen Mädchen/Frauen und Jungen/Männern vorhanden (z. B. McElvany et al., 2017; Stanat \& Kunter, 2002). Ähnlich dem Aspekt der Lesekompetenzunterschiede, stammen hier ebenfalls viele Befunde aus der Altersgruppe von Schülerinnen und Schülern. Zum Freizeitleseverhalten vom Erwachsenen gibt es bisher deutlich weniger Studien (z. B. Charlton et al., 2004; Schreier \& Odağ, 2004).

Mittels eines vertieften Verständnisses für unterschiedlichen Lesepräferenzen, Themengebiete sowie inhaltliche Interessen, sollte es infolgedessen möglich sein, die Lesekompetenz von Mädchen/Frauen und Jungen/Männern gezielter zu fördern (McGeown, 2015). Obwohl keine klare Kategorisierung von Genres, Leseanforderungen sowie Inhalten vorliegt und diese, wie auch das Leseverhalten, sehr divers und facettenreich sind, so ist doch ersichtlich, dass Frauen und Männer im Allgemeinen verschiedene Lektüreinhalte präferieren: Mädchen/Frauen lesen in ihrer Freizeit häufiger zur Unterhaltung, um dem Altag zu entfliehen und um sich mit Charakteren aus dem Gelesenen zu identifizieren. Jungen/Männer hingegen haben im Allgemeinen eher Präferenzen für Inhalte, die sie 
informieren und durch die sie Wissen generieren können (Charlton et al., 2004; Philipp, 2011; Schreier \& Odağ, 2004). Somit wird auf der theoretischen Grundlage zur „Funktion des Lesens“"von Groeben (2004) angenommen, dass Frauen häufiger mit der Intention lesen, sich zu unterhalten und deswegen eher fiktionale Texte, wie Romane, Krimis oder ScienceFiction wählen. Männer hingegen lesen häufiger, um sich zu informieren. Daher präferieren sie im Allgemeinen eher Texte mit informativem Inhalt, wie Zeitungen und Sachbücher.

Des Weiteren wurde im vorangegangenen Kapitel 3 sowie in der Ausformulierung der Forschungsfrage 1 die Entwicklung von Lesekompetenzunterschieden zwischen Frauen und Männern eingehend beschrieben. Wie die Zusammenschau der Ergebnisse in dieser Arbeit verdeutlicht, war die Untersuchung der Entwicklung der Lesekompetenz von Erwachsenen bisher im geringeren Maß im Fokus. Viele der in dieser Arbeit erwähnten Ergebnisse beziehen sich primär auf die Lesekompetenz von Kindern und Jugendlichen.

Gleichzeitig soll an dieser Stelle nochmals erwähnt werden, dass die Messung der Lesekompetenz in den jeweiligen Studien und zwischen den verschiedenen Alterskohorten auf unterschiedlichen konzeptionellen Umsetzungen fußt. So konnten Solheim \& Lundetræ (2017) in einer Gegenüberstellung der Lese-Assessments von PIRLS 2011, PISA 2009 und PIAAC 2012 unter anderem aufzeigen, dass im Framework von PIAAC keine narrativen Texte verwendet werden, sondern ausschließlich Sachtexte zur Messung der Lesekompetenz bei Erwachsenen.

Genauere Untersuchungen $\mathrm{zu}$ den Textsorten und -formen bei den teilnehmenden 15Jährigen in der PISA-Studie konnten hingegen, wie im Kapitel 4.3 erläutert, Hinweise auf Stärken und Schwächen in der Lesekompetenz vom jungen Frauen und Männern geben (Artelt \& Schlagmüller, 2004; Stanat \& Kunter, 2002). Bei erwachsenen Leserinnen und Lesern wurde allerdings die textsortenspezifische Lesekompetenz (Sachtexte vs. literarische Texte) bislang nicht detailliert analysiert, weshalb vorhandene Stärken und Schwächen in den verschiedenen Textsorten noch nicht eruiert werden konnten.

Auf Grundlage dieser Forschungslücke kann die zweite Forschungsfrage für die vorliegende Arbeit formuliert werden.

Anhand eines Mediationsmodells wird analysiert, ob Frauen und Männer bei der Auswahl ihrer Freizeitlektüre unterschiedliche Präferenzen hinsichtlich der Leseintention haben. Es 
wird angenommen, dass Frauen häufiger zur Unterhaltung und Männer häufiger zur Information lesen.

Des Weiteren wird bei Erwachsenen nicht die allgemeine Lesekompetenz untersucht, sondern eine textsortenspezifische Lesekompetenz genauer betrachtet. Es wird erwartet, dass sich hinsichtlich der Lesekompetenz in Sachtexten und literarischen Texten Divergenzen in Abhängigkeit der Lesepräferenzen zeigen. Dahingehend, dass sich Lesepräferenzen im Lesen zur Unterhaltung oder dem Lesen zur Informationsaufnahme auf die textsortenspezifische Lesekompetenz in Sachtexten und literarischen Texten auswirken.

Die zweite Forschungsfrage lautet somit:

Gibt es Unterschiede zwischen Männern und Frauen in den Lesepräferenzen sowie in der textsortenspezifischen Lesekompetenz bei Sachtexten und literarischen Texten, und welchen Einfluss haben die Lesepräferenzen auf die textsortenspezifische Lesekompetenz bei Sachtexten und literarischen Texten?

\subsection{Forschungsfrage 3: Merkmal auf Seiten der Texte}

Bezogen auf die differenzielle Erklärung von Lesekompetenzunterschieden zwischen Frauen und Männern zeigen die Forschungsergebnisse, dass nicht nur Determinanten der Lesenden von Relevanz sind, sondern gleichfalls die Struktur und Inhalte von Texten (z. B. Artelt \& Schlagmüller, 2004; Stanat \& Kunter, 2002). Wie umfassend in Kapitel 4.3 anhand des Tetraedermodells von Artelt und Kolleginnen sowie Kollegen (2007) erläutert, setzt sich die Text-Lesenden-Interaktion aus dem Zusammenspiel der Leseziele bzw. der Lesepräferenz und dem jeweiligen Inhalt der gewählten Texte zusammen. Genau dieser Textinhalt könnte für die Erklärung von Geschlechtsunterschieden von Bedeutung sein und Varianz in der Lesekompetenz von Männern und Frauen aufklären.

Abbildung 1 visualisiert, dass die soziale Umwelt einen umspannenden Rahmen für die Text-Lesenden-Interaktion darstellt. Hierdurch wird verdeutlicht, welchen Einfluss die soziale Umwelt sowohl auf die Einstellung der Lesenden in Bezug auf das Lesen und das Leseverhalten als auch auf den Inhalt und die verwendete Sprache in den Texten hat. 
Außerdem existieren stabile, sozial geteilte Annahmen darüber, wie Frauen und Männer typischerweise in ihrem Verhalten sein sollten oder sich auch tatsächlich verhalten (d. h. Geschlechtsstereotype). Vorangehend wurde die Annahme in Kapitel 4.1 dargestellt, dass über Geschlechtsstereotype die Motivation und das individuelle Interesse für ein bestimmtes Thema beeinflusst werden (Möller \& Schiefele, 2004). Ein hohes Interesse an einem Thema führt zu positiven Assoziationen, welche eine wichtige Motivationsquelle für die Fortsetzung der Aktivität sein kann. Basierend darauf verfolgen Frauen und Männer im Allgemeinen andere Interessen und sammeln unterschiedliches Vorwissen aus verschiedenen Erfahrungs- und Inhaltsbereichen (z. B. Bildung, Beruf, Freizeitaktivitäten; Baron, Markman \& Hirsa, 2001; Christin, 2012; Lagaert, van Houtte \& Roose, 2017; Nosek, Banaji \& Greenwald, 2002). Demnach sollte es dazu kommen, dass Frauen und Männer sich mit anderen Themenfeldern auseinandersetzen und unterschiedliches Vorwissen zu verschiedenen Inhalten haben.

Des Weiteren ist anzunehmen, dass Geschlechtsstereotype über die verwendete Sprache vermittelt werden, infolgedessen Ungleichheiten zwischen Frauen und Männern aufrechterhalten werden (u. a. Garnham et al. 2016). Bereits im Wortschatz von Kindern konnten stereotype Zuordnungen des Geschlechts mit einem Wort verknüpft werden (z. B. Emotionen, Fürsorge für Mädchen und Stärke, Abenteuer für Jungen; El-Khechen et al., 2017; McElvany et al., 2016). Ähnlich wie im Wortschatz, könnte möglicherweise das Vorhandensein von Geschlechtsstereotypen in Inhalten des Textmaterials zur Betonung von Stereotypen führen und so einen Einfluss auf die Lesekompetenz von Frauen und Männern haben. Folglich könnte es sein, dass Männer in einem Text, welcher eher stereotyp männliche Inhaltsbereiche thematisiert, eine höhere Lesekompetenz haben als Frauen. Umgekehrt könnten Frauen in eher stereotyp weiblichen Inhaltsbereichen eine bessere Lesekompetenz haben als Männer. Ferner, so legt es das Tetraedermodell von Artelt und Kolleginnen sowie Kollegen (2007) nahe, erleichtert das Vorhandensein von Vorwissen zu einem bestimmten Textinhalt dem Leser oder der Leserin das Herstellen von lokaler und globaler Kohärenz sowie den Aufbau eines Situationsmodells. Durch ein möglicherweise fehlendes Vorwissen und der fehlenden Vertrautheit mit einem bestimmten Thema und der gleichzeitigen Konfrontation mit einem geschlechtsstereotypen Textinhalt, welcher mit der anderen Geschlechtsgruppe assoziiert ist (z. B. Yoga ist eine eher weibliche Sportart, für die sich mehr Frauen als Männer interessieren), könnte es beim Leser zur Verringerung der wahrgenommenen Kompetenz mit dem Thema kommen (d. h. Stereotyp Threat; z. B. 
Martiny \& Götz, 2011). Dieses Zusammenspiel aus Vorwissen und dem spezifischen Interesse, könnte einen Einfluss auf das Bearbeiten und das Verständnis von geschlechtsstereotypen Textinhalten haben und sich in der Lesekompetenz von Frauen und Männern widerspiegeln.

Auf Grundlage dieser Annahmen kann die dritte Forschungsfrage für die vorliegende Arbeit formuliert werden.

Wie eingangs angeführt ist das zweite übergeordnete Ziel der Forschungsarbeit Determinanten zu betrachten, welche Unterschiede in der Lesekompetenz von Frauen und Männern erklären können. Über das Vorhandensein und die Weitergabe von Geschlechtsstereotypen hat die soziale Umwelt einen Einfluss auf das Verhalten und Interessen von Frauen und Männern. Daher wird untersucht, ob ein geschlechtsstereotyp konnotierter Textinhalt einen Einfluss auf die Lesekompetenz von Frauen und Männern hat. In Abhängigkeit davon, ob der Inhalt eines Textes eher stereotyp weiblich oder eher stereotyp männlich konnotiert ist, wird vermutet, dass dieser differenziell die Lesekompetenz von Männern und Frauen beeinflusst.

Die dritte Forschungsfrage lautet somit:

Können geschlechtsstereotype Textinhalte Unterschiede in der Lesekompetenz von Frauen und Männern erklären? 


\section{Darstellung der zentralen Befunde der einzelnen Beiträge}

Vorangestellt erfolgt in Tabelle 1 eine Übersicht für die, in dieser Synopse dargestellten Beiträge. Daran anschließend erfolgen für jeden Beitrag einzeln eine Zusammenfassung des methodischen Vorgehens sowie die Darstellung der zentralen Befunde.

Tabelle 1:

Übersicht der drei Beiträge der vorliegenden Synopse

\begin{tabular}{llll}
\hline $\begin{array}{l}\text { Autorinnen/ } \\
\text { Autoren }\end{array}$ & $\begin{array}{l}\text { Ver- } \\
\text { offent } \\
\text { licht }\end{array}$ & Titel des Beitrags & Publikation in \\
\hline Thums, Kathrin & & When do gender differences in Eingereicht in \\
Schwabe, Franziska & & reading competence diminish? A Scientific Studies \\
Sengewald, Marie-Ann & & longitudinal study of women and of reading \\
Artelt, Cordula & & men from adolescence to young & \\
Wolter, Ilka & adulthood.
\end{tabular}

Thums, Kathrin

Reading for entertainment or European Journal

Artelt, Cordula information reception? Gender of Psychology of

Wolter, Ilka

differences in reading pre- Education

ferences and their impact on text- doi:10.1007/s 102

type-specific reading com- 12-020-00486-1

petences in adult readers

Thums, Kathrin

2020 The impact of gender- Zeitschrift für

Gnambs, Timo stereotypical text contents on Erziehungs-

Wolter, Ilka reading competence in women and men wissenschaft, 23(6), 1283-1301. doi.org/10.1007/s

Der Einfluss von ge- 11618-020schlechtsstereotypen Textin- 00980-8 halten auf die Lesekompetenz von Frauen und Männer 


\subsection{Beitrag 1: When do gender differences in reading competence diminish? A longitudinal study of reading competence of women and men from adolescence to young adulthood}

\subsubsection{Hypothesen}

Vor dem Hintergrund der fehlenden Ergebnisse zur Entwicklung von

Geschlechtsunterschieden in der Lesekompetenz im Übergang ins junge Erwachsenenalter, fokussiert Beitrag 1, basierend auf der ersten Forschungsfrage, die Lesekompetenzentwicklung von jungen Frauen und Männern im Alter von 16 bis 22 Jahren.

Dazu wurden folgende Hypothesen formuliert:

1. Es wurde angenommen, dass sich Geschlechtsunterschiede im Lesen mit der Zeit verringern.

In der neunten Klasse wurden Unterschiede in der Lesekompetenz zwischen den Jugendlichen erwartet. Diese Unterschiede sollten sich jedoch bis ins junge Erwachsenenalter verringern.

2. Für die Veränderung der Lesekompetenz wurde ein differenzieller Verlauf für junge Frauen und Männern erwartet. Der Verlauf der Lesekompetenz sollte sich dahingehend unterscheiden, dass junge Männer in ihrer Lesekompetenz erst zu einem späteren Zeitpunkt zu den jungen Frauen aufholen.

\subsubsection{Methode}

\subsubsection{Stichprobe}

Die Studie wurde mit Daten der Startkohorte Klasse $9(\mathrm{SC} 4)^{3}$ des Nationalen Bildungspanels (NEPS; Blossfeld, Roßbach \& von Maurice, 2011) durchgeführt. Die analysierte Stichprobe

\footnotetext{
Es wurden Daten des Nationalen Bildungspanels (NEPS) genutzt: Starkohorte Klasse 9 (doi:10.5157/NEPS:SC4:10.0.0). Die Daten des NEPS wurden von 2008 bis 2013 als Teil des Rahmenprogramms zur Förderung der empirischen Bildungsforschung erhoben, welches vom Bundesministerium für Bildung und Forschung (BMBF) finanziert wurde. Seit 2014 wird NEPS vom Leibniz-Institut für Bildungsverläufe e.V. (LIfBi) an der Otto-Friedrich-Universität Bamberg in Kooperation mit einemdeutschlandweiten Netzwerk weitergeführt.
} 
zur Beantwortung der Hypothesen enthielt 14,502 Schülerinnen und Schüler der neunten Klassenstufe, davon waren 7,226 weiblich (49.8 \%) und 7,276 männlich (50,2 \%). Im Schnitt waren die Teilnehmenden zum ersten Messzeitpunkt 15.72 Jahre alt ( $\mathrm{SD}=0.72$; Spanne von 12 - 19 Jahre). Von 3,595 Studienteilnehmerinnen und -teilnehmern (24.8\%) wurde ein Familienmitglied (Zielperson, Mutter oder Vater) nicht in Deutschland geboren. Von 126 Teilnehmenden (0.9 \%) waren keine Angaben zu ihrem Geburtsland bekannt.

Von den Teilnehmenden besuchten zum ersten Messzeitpunkt 5,356 Schülerinnen und Schüler (36.9 \%) in der neunten Klassenstufe das Gymnasium. Die anderen 8,991 Schülerinnen und Schüler besuchten nicht-gymnasiale Schulzweige (u. a. Hauptschule, Realschule). Von 155 Schülerinnen und Schüler (1.1\%)wurde keine Angabe zur Schulform gemacht.

\subsubsection{Instrument}

Lesekompetenz: Zum ersten Messzeitpunkt (T1, Herbst/Winter 2010) der Studie befanden sich alle Schülerinnen und Schüler in der neunten Klassenstufe. Zu den Messzeitpunkten T2 (Welle 2, Frühling 2011, Schultestung, Ende 9. Klasse), T3 (Welle 7, 2013/2014, Schultestung in Klasse 12 und Individualfeld) und T4 (Welle 10, 2016/2017, Individualfe ld) wurde die Lesekompetenz der Teilnehmenden erfasst. Die Messung der Lesekompetenz der Teilnehmenden fußt auf der Rahmenkonzeption zum NEPS-Lesekompetenztest (Gehrer, Zimmermann, Artelt \& Weinert, 2013). Zur Messung der Lesekompetenz werden im NEPSLesekompetenztest fünf kontinuierliche Textsorten verwendet (Sachtexte, kommentierende Texte, literarische Texte, Anleitungen und Werbetexte). Durch verschiedene kognitive Verstehensanforderungen (a. Informationen im Text ermitteln, b. textbezogene Schlussfolgerungen ziehen, c. Reflektieren und Bewerten) der jeweiligen, auf die Texte bezogene Aufgaben, wird die Lesekompetenz operationalisiert.

Die Skalierung der Lesekompetenz basiert auf einer IRT-Skalierung (Item-ResponseTheorie) und für jeden Studienteilnehmer und jede Studienteilnehmerin wird ein für den Längsschnitt verlinkter Weighted Maximum Likelihood Schätzer (WLE) zur Verfügung gestellt (zum Linking: Fischer, Rohm, Gnambs \& Carstensen, 2016; SC4 Lesen: Gnambs, Fischer \& Rohm, 2017; Haberkorn, Pohl, Hardt \& Wiegand, 2012; mehr zur KompetenzSkalierung im NEPS siehe Pohl \& Carstensen, 2013). Zu den Messzeitpunkten T2 und T3 wurde die Lesekompetenz papierbasiert erfasst. Zu T4 erfolgte eine Umstellung auf eine computerbasierte Testung (Rohm, Scharl, Ettner \& Gehrer, 2019). 


\subsubsection{Analyse und Auswertungsmethoden}

Die Analyse der Lesekompetenz im Längsschnitt und deren differenzielle Entwicklung für junge Frauen und Männer wurde mittels eines Neighbour-Change-Modell berechnet (Steyer, Eid \& Schwenkmezger, 1997; Steyer, Partchev \& Shanahan, 2000). Im Vergleich zu einem Wachstumskurvenmodell bietet das Differenzmodell den Vorteil, dass nicht von einer linearen Entwicklung der Kompetenz ausgegangen wird.

Das Geschlecht der Teilnehmenden wurde zu T1 von der jeweiligen Schule durch eine Liste aller teilnehmenden Schülerinnen und Schülern angegeben. Wie bereits erwähnt, wird vorliegend eine dichotome Einteilung des Geschlechts vorgenommen $(0=$ Frauen; $1=$ Männer). Im Modell wurde außerdem im Hinblick auf die soziodemographischen Merkmale (Bildungshintergrund der Eltern, Migrationshintergrund der Teilnehmenden oder eines Elternteils, besuchte Schulform in Klasse Neun) sowie für ein kognitives Merkmal (allgemeine kognitive Grundfähigkeit; Brunner, Lang \& Lüdtke, 2014) der Teilnehmenden kontrolliert.

Zum Umgang mit fehlenden Werten wurde eine Full Information Maximum Likelihood (FIML) Schätzung angewandt (Allison, 2009; Zinn \& Gnambs, 2018). Das Modell wurde mit der Software Mplus berechnet (Version 8; Muthén \& Muthén, 2017).

\subsubsection{Zentrale Befunde}

Die Ergebnisse des Differenzmodells zeigten wie erwartet, dass die jungen Frauen im ersten Messzeitpunkt der Lesekompetenz, mit ca. 16 Jahren (T2), eine höhere Lesekompetenz $(d=-0.26)$ im Vergleich zu den jungen Männern aufwiesen. Dieser Geschlechtsunterschied im Lesen war ebenfalls im nächsten Messzeitpunkt (T3; 18 Jahre) vorhanden, verringerte sich jedoch leicht $(d=-0.22)$. Demgegenüber lag zum letzten Messzeitpunkt (T4; 22 Jahre) kein Unterschied in der Lesekompetenz von jungen Frauen und Männern mehr vor $(d=0.00)$.

Die Befunde konnten die Annahmen aus Hypothese 1 bestätigen, dass sich die Geschlechtsunterschiede im Zeitverlauf verringern. So kann bei den Jugendlichen in der neunten Klasse noch ein deutlicher Unterschied in der Lesekompetenz festgestellt werden. Hingegen gibt es keinen Unterschied mehr in der Lesekompetenz von jungen Männern und Frauen nach dem Verlassen der Schule. 
Durch das Neighbour-Change-Modell konnte ein differenzieller, nicht linearer Verlauf der Lesekompetenz für die jungen Frauen und Männer festgestellt werden. Demzufolge war bei den jungen Frauen zwischen 16 und 18 Jahren eine signifikante Zunahme in der Lesekompetenz feststellbar. Diese stagnierte in der Folge und stieg im Alter zwischen 18 und 22 Jahren nicht weiter an. Die jungen Männer hingegen konnten über den gesamten Zeitverlauf ihre Lesekompetenz verbessern. Sowohl zwischen T2 und T3 als auch zwischen T3 und T4 kam es zu einer signifikanten Zunahme in der Lesekompetenz. Der Zuwachs bei den jungen Männern fiel dabei zwischen 16 und 18 Jahren größer aus als zwischen 18 und 22 Jahren.

Somit liegt, wie in Hypothese 2 angenommen, eine differenzielle Entwicklung der Lesekompetenz für junge Frauen und Männer vor. Ausgangspunkt war dabei eine geringere Lesekompetenz bei den jungen Männern mit 16 Jahren als bei den jungen Frauen. Hinsichtlich der Entwicklung zeigte sich bei den jungen Männern ein höherer und linearer Anstieg in der Lesekompetenz im Vergleich zum nicht linearen und leicht stagnierenden Verlauf der Lesekompetenz von jungen Frauen.

\subsection{Beitrag 2: Reading for entertainment or information reception? Gender differences in reading preferences and their impact on text- type-specific reading competences in adult readers}

\subsubsection{Hypothesen}

Wie in der zweiten Forschungsfrage formuliert, wird vor dem Hintergrund der fehlenden Ergebnisse zur textsortenspezifischen Lesekompetenz in literarischen Texten und Sachtexten bei erwachsenen Frauen und Männern sowie dem positiven Einfluss des Freizeitleseverhaltens auf die Lesekompetenz, in Beitrag 2 erwartet, dass sich unterschiedliche Lesepräferenzen in der Freizeit von Frauen und Männern auf die textsortenspezifische Lesekompetenz auswirken. Darüber hinaus wird angenommen, dass Geschlechtsunterschiede in der Lesekompetenz in literarischen Texten und Sachtexten durch geschlechtsspezifische Lesepräferenzen mediiert werden. 
Dazu wurden folgende Hypothesen formuliert:

1. Es wurden geschlechtsspezifische Unterschiede in der Lesekompetenz bei literarischen Texten und Sachtexten angenommen: Frauen sollten im Durchschnitt eine höhere textsortenspezifische Lesekompetenz in literarischen Texten erzielen und Männer im Durchschnitt eine höhere textsortenspezifische Lesekompetenz in Sachtexten.

2. Der Einfluss der Lesepräferenzen auf die textsortenspezifische Lesekompetenz wurde wie folgt angenommen:

a) Personen, die häufiger zur Unterhaltung lesen, sollten eine höhere Lesekompetenz in literarischen Texten haben als in Sachtexten.

b) Hingegen sollten Personen, die häufiger zur Informationsaufnahme lesen, eine höhere Lesekompetenz in Sachtexten haben als in literarischen Texten.

3. Außerdem wurde vermutet, dass sich Geschlechtsunterschiede in den Lesepräferenzen zeigen: Frauen sollten hinsichtlich ihrer Lesepräferenzen generell häufiger zur Unterhaltung lesen, Männer hingegen häufiger zum Zweck der Informationsaufnahme.

\subsubsection{Methode}

\subsubsection{Stichprobe}

Als Datengrundlage zur Beantwortung der Hypothesen wurde eine Entwicklungsstudie des Nationalen Bildungspanels (NEPS; Blossfeld et al., 2011) verwendet. Die finale Stichprobe umfasste 830 Erwachsene zwischen 19 - 71 Jahren $(M=31.04 ; S D=12.53)$, davon waren 51.9 \% Frauen $(n=431)$ und $48.1 \%$ Männer $(n=399)$. Zur Erprobung von neuen Texten für die NEPS-Hauptstudie wurde speziell für diese Stichprobe die Gruppe von Studierenden überproportional berücksichtigt. Daher gaben überdurchschnittlich viele Teilnehmende als höchsten Bildungsabschluss ein Abitur an $(n=534 ; 64.3 \%)$. Auf Grund der geringen Anzahl an Teilnehmenden mit einem Migrationshintergrund $(n=70)$ wurden nur Personen für die Untersuchung der Fragestellung berücksichtigt, welche bestätigten keinen Migrationshintergrund zu haben und als Erstsprache Deutsch sprechen. 


\subsubsection{Instrumente}

Lesekompetenz: Zur Messung der textsortenspezifischen Lesekompetenz wurden drei literarische Texte und sechs Sachtexte basierend auf der Konzeption der NEPSLesekompetenztests (Gehrer et al., 2013) berücksichtigt.

Anders als die NEPS-Rahmenkonzeption zur Kompetenzskalierung (Pohl \& Carstensen, 2013), wurde die Lesekompetenz in einem 2-PL-Modell (2 parameter logistic model; Birnbaum, 1968) skaliert und neben der Personenfähigkeit und der Aufgabenschwierigke it die Trennschärfe der Items berücksichtigt. Für die Schätzung der Lesekompetenz konnten 37 Aufgaben in den Sachtexten (IRT-Reliabilität $=.70$ ) und 17 Aufgaben für die literarischen Texte (IRT-Reliabilität $=.60$ ) berücksichtigt werden. Für jede Person wurde für die jeweilige Textsorte ein WLE (Weighted Maximum Likelihood Schätzer) geschätzt.

Lesepräferenzen: Die Lesepräferenz der Studienteilnehmerinnen und Studienteilnehmer wurde innerhalb eines Fragebogens auf einer fünfstufigen Skala (nie oder selten bis täglich) mit der Frage erhoben: „Wie oft lesen Sie in Ihrer Freizeit normalerweise ...“. Abgefragt wurden verschiedene Genres, im Einzelnen Krimis, Tages- und Wochenzeitungen, Sachbücher, Biografien, Science-Fiction, Romane und Thriller oder Horrorbücher.

Auf Basis der theoretischen Ausführungen zu „Funktionen des Lesens“ nach Groeben (2004), wurden ein formativer Index der Präferenzen des Lesens zur Unterhaltung (Krimis, Science-Fiction, Romane und Thriller oder Horrorbücher) sowie aus Informationsgrün den (Tages- und Wochenzeitungen, Sachbücher, Biografien) gebildet.

\subsubsection{Analyse und Auswertungsmethoden}

In einem Mediationsmodell wurde die Lesekompetenz in literarischen Texten und Sachtexten als abhängige Variable, die Lesepräferenzen zur Unterhaltung oder Informationsaufnahme als Mediatorvariablen und das Geschlecht der Teilnehmenden als Prädiktor berechnet.

Die Anzahl der Bücher im Haushalt sowie das Alter der Teilnehmenden wurden dabei als Kontrollvariablen im Modell berücksichtigt.

Um die Signifikanz der vier indirekten Pfade im Mediationsmodell zu testen, wurde die Methode des Bootstrapping mit 1000 Bootstrapp Stichproben angewendet (siehe MacKinnon, Cheong \& Pirlott, 2012). 
Zum Umgang mit fehlenden Werten wurde eine Full Information Maximum Likelihood (FIML) Schätzung angewandt (Allison, 2009; Zinn \& Gnambs, 2018). Das Modell wurde mit der Software Mplus berechnet (Version 8; Muthén \& Muthén, 2017).

\subsubsection{Zentrale Befunde}

Durch das Mediationsmodell konnten nach der Berücksichtigung der Lesepräferenzen sowie der Kontrollvariablen kleine geschlechtsspezifische Unterschiede von Erwachsenen in deren Lesekompetenz in literarischen Texten und Sachtexten festgestellt werden. Männer hatten eine etwas höhere Lesekompetenz in Sachtexten als Frauen $(d=0.14)$. In literarischen Texten zeigte sich demgegenüber kein Unterschied in der Lesekompetenz von erwachsenen Frauen und Männern.

Demnach konnte die erste Hypothese dieser Untersuchung in Teilen dahingehend bestätigt werden, dass Männer im Durchschnitt eine vergleichsweise etwas höhere textsortenspezifische Lesekompetenz hinsichtlich von Sachtexten haben als Frauen. Jedoch konnte nicht bestätigt werden, dass Frauen im Vergleich zu Männern im Durchschnitt eine höhere textsortenspezifische Lesekompetenz bei literarischen Texten haben.

Mit der zweiten Hypothese sollte der Einfluss der Lesepräferenzen auf die textsortenspezifische Lesekompetenz untersucht werden. Es zeigte sich in den Ergebnissen, dass Personen, die zur Unterhaltung lesen, eine etwas höhere Lesekompetenz in literarischen Texten $(d=0.14)$ haben, während das Lesen zur Unterhaltung keinen signifikanten Einfluss auf die Lesekompetenz in Sachtexten hat.

Für die Lesepräferenz zur Informationsaufnahme zeigte sich ein signifikant gleich großer Einfluss auf die Lesekompetenz in Sachtexten $(d=0.27)$ und literarischen Texten $(d=0.27)$. Daher konnte Hypothese zwei nur teilweise bestätigt werden. Erwachsene Personen, die häufiger zur Unterhaltung lesen, haben folglich eine etwas höhere Lesekompetenz in literarischen Texten als in Sachtexten. Erwachsene Personen, die häufiger zur Informationsaufnahme lesen, haben indes nicht nur in Sachtexten eine höhere Lesekompetenz, sondern ebenso in literarischen Texten.

Hypothese drei, wonach geschlechtsspezifische Unterschiede in den Lesepräferenzen angenommen wurden, konnte bestätigt werden. Frauen lesen im Durchschnitt häufiger zur 
Unterhaltung (Krimis, Science-Fiction, Romane sowie Thriller oder Horrorbücher; $d=$ 0.34) als Männer. Hingegen bevorzugen Männer im Vergleich zu Frauen häufiger informierende Genres (Tages- und Wochenzeitungen, Sachbücher, Biografien; $d=-0.23$ ).

Für die getesteten indirekten Effekte des Geschlechts, vermittelt über die Lesepräferenzen auf die textsortenspezifischen Lesekompetenzen, ergaben sich für beide Pfade über das Lesen zur Informationsaufnahme signifikante kleine Effekte $(d=0.17)$ für beide Textsorten. Hingegen zeigten sich keine signifikanten indirekten Effekte vermittelt durch die Lesepräferenz zur Unterhaltung auf die textsortenspezifische Lesekompetenz in Sachtexten und literarischen Texte von Frauen und Männern.

\subsection{Beitrag 3: The impact of gender-stereotypical text contents on reading competences in women and men}

\subsubsection{Hypothesen}

Basierend auf der dritten Forschungsfrage wird in Beitrag 3 erwartet, dass geschlechtsstereotype Textinhalte einen Einfluss auf die Lesekompetenz von Frauen und Männer haben. Es wird angenommen, dass über die Gesamtinhalte von Texten Geschlechtsunterschiede in der Lesekompetenz erklärt werden können. In Abhängigkeit davon, ob der Inhalt eines Textes eher stereotyp weiblich oder eher stereotyp männlich konnotiert ist, sollte dieser differenziell die Lesekompetenz von Männern und Frauen beeinflussen.

Dazu wurden folgende Hypothesen formuliert:

1. Neben einem Generalfaktor der Lesekompetenz mit substantiellen Faktorladungen der Items für eine eindimensionale Skala sollten noch zwei weitere geschlechtsspezifische Faktoren der Lesekompetenz identifiziert werden. Diese geschlechtsspezifischen Faktoren der Lesekompetenz sollten in

a) geschlechtsstereotyp weibliche Textinhalte und

b) geschlechtsstereotyp männliche Textinhalte differenziert werden können. 
2. Abhängig von Hypothese 1 wurden Unterschiede in den geschlechtsspezifischen Faktoren der Lesekompetenz zwischen Frauen und Männern erwartet:

a) Frauen sollten im Vergleich zu Männern im Durchschnitt eine höhere Lesekompetenz in geschlechtsstereotyp weiblichen Textinhalten erzielen.

b) Männer sollten im Vergleich zu Frauen im Durchschnitt eine höhere Lesekompetenz in geschlechtsstereotyp männlichen Textinhalten erreichen.

Anzumerken ist, dass sich die Hypothesen auf verschiedene Analyseebenen beziehen. Die erste Hypothese bezieht sich auf das Textmaterial zur Messung der Lesekompetenz und den Inhalt der Texte. Die zweite Hypothese legt den Fokus auf individuelle Unterschiede in den geschlechtsstereotypen Faktoren der Lesekompetenz zwischen Frauen und Männern. Im Speziellen geht es hier um die Interaktion zwischen Merkmalen der Lesenden und den Texteigenschaften.

\subsubsection{Methode}

\subsubsection{Stichprobe}

Für die dritte Fragestellung wurde ebenfalls die in Beitrag 2 beschriebene Entwicklungsstudie des Nationalen Bildungspanels (NEPS; Blossfeld et al., 2011) verwendet.

Die Stichprobe umfasste eine sehr große Spannbreite des Altersbereichs (19 - 71 Jahren). Mit der Begründung der Vermutung anderer Geschlechtsstereotype für ältere Geburtskohorten (Athenstaedt, 2000; Eagly, Nater, Miller, Kaufmann \& Sczesny, 2019; Haines, Deaux \& Lofaro, 2016), wurden alle Personen von der Fragestellung ausgeschlossen, die vor 1950 geboren waren.

Daher umfasste die finale Stichprobe für diese Fragestellung 813 Erwachsene zwischen 19 bis 65 Jahren $(M=30.08 ; S D=11.16)$, davon 424 Frauen $(52.2 \%)$.

\subsubsection{Instrumente}

Lesekompetenz: Aus der NEPS-Entwicklungsstudie wurden sechs Sachtexte, drei literarische Texte und drei Werbetexte berücksichtigt. Insgesamt umfassten alle zwölf kontinuierlichen Texte 68 Aufgaben in einem geschlossenen dichotomen oder komplexen (z. B. Entscheidungs- und Zuordnungsaufgaben) Multiple-Choice Format. Die kognitiven 
Anforderungen der Aufgaben (a. Informationen im Text ermitteln, b. textbezogene Schlussfolgerungen ziehen und c. Reflektieren und Bewerten) wurden in allen Textsorten realisiert (siehe dazu Gehrer et al., 2013).

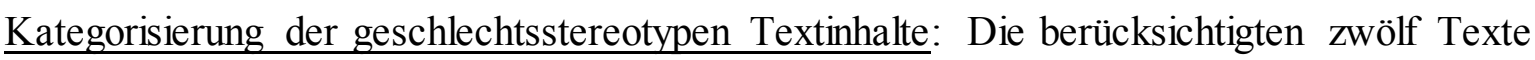
wurden a priori in einem zweistufigen Verfahren hinsichtlich ihres geschlechtsstereotypen Gesamtinhalts in geschlechtsstereotype weibliche, männliche und geschlechtsneutrale Textinhalte kategorisiert.

Im ersten Schritt der Kategorisierung wurden die oben beschriebenen Merkmale aus psychologisch-pädagogischen Studien abgeleitet, um typisch weibliche und männliche Attribute, Interessen, Berufe und Aktivitäten beschreiben zu können (u. a. Athenstaedt \& Alfermann, 2011; Christin, 2012; Haines et al., 2016). Im zweiten Schritt wurden die Texte inhaltlich nach geschlechtsstereotyp weiblichen, männlichen oder neutralen Konnotationen von drei Expertinnen des Forschungsbereiches kategorisiert. Die Kategorisierung der Textinhalte erfolgte auf einer kontinuierlichen Antwortskala mit fünf Antwortoptionen (sehr typisch männlicher, eher typisch männlicher, geschlechtsneutraler, eher typisch weiblicher, sehr typisch weiblicher Textinhalt).

Im Anschluss an die Kategorisierung der Texte konnten jeder Textkonnotation (geschlechtsstereotyp weibliche und männliche sowie geschlechtsneutrale) vier Texte zugeordnet werden (zwei Sachtexte, ein literarischer Text und ein Werbetext).

Ein Beispiel für einen geschlechtsstereotyp weiblichen Textinhalt ist dabei ein Text aus dem Lesekompetenztest über die Sportart Yoga und verschiedene Yoga-Traditionen. Yoga wurde als typisch weibliche Sportart kategorisiert, welche von mehr Frauen als Männern betrieben wird und daher sollten das Interesse und das Vorwissen für diese Sportart bei Frauen höher sein als bei Männern. Ein Beispiel für einen geschlechtsstereotyp männlichen Textinhalt ist ein Text über eine bestimmte Technik der Fotographie, bei der viele technische Details zur Kamera und dem zugrundeliege nden technischen Verfahren beschrieben werden.

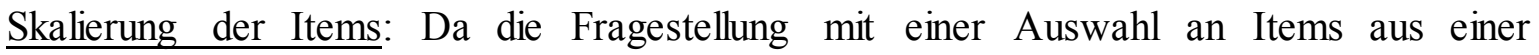
Entwicklungsstudie beantwortet wurde, mussten die vorhandenen Daten zur weiteren Bearbeitung vorab skaliert werden. Auf Basis des 2PL Modells (Birnbaum, 1968) wurden 68 Aufgaben zur Bestimmung der Lesekompetenz hinsichtlich ihrer Qualität ausgewählt. Es 
wurde eine Differential Item Functioning (DIF) Analyse zur Bestimmung der Funktionsweise der Items für Frauen und Männer durchgeführt (Pohl \& Carstensen, 2013).

Nach der Skalierung ergab sich für die drei geschlechtsstereotypen Textkonnotationen eine vergleichbare Auswahl der Aufgaben hinsichtlich der Wortanzahl, Reliabilität sowie mittleren Itemschwierigkeit.

\subsubsection{Analyse und Auswertungsmethoden}

Zur Bestimmung der dimensionalen Struktur der Lesekompetenzaufgaben (Hypothese 1) wurde ein ordinales Bi-Faktor-Modell geschätzt. Neben dem Generalfaktor der Lesekompetenz wurden zwei weitere spezifische Faktoren hinsichtlich der geschlechtsstereotypen Textkonnotationen angenommen.

Für den Generalfaktor der Lesekompetenz sollten alle 68 Items eine substantielle Ladung $(\beta \geq .40)$ aufweisen. Darüber hinaus sollten die spezifischen Faktoren der geschlechtsstereotyp weiblichen und geschlechtsstereotyp männlichen Textinhalte spezifische Varianzen an der Lesekompetenz aufklären. Es wurde erwartet, dass die Items der geschlechtsstereotyp weiblichen Textinhalte (insgesamt 23 Items) eine substantielle Faktorladung haben würden und einen geschlechtsspezifischen Faktor für geschlechtsstereotype weibliche Textinhalte identifizieren könnten. Ebenso sollten die Items der geschlechtsstereotyp männlichen Textinhalte (insgesamt 24 Items) auf einen geschlechtsspezifischen Faktor für geschlechtsstereotype männliche Textinhalte laden. Die Items der neutralen Textinhalte (insgesamt 21 Items) stellten die Referenzkategorie im Modell dar.

Um die Unterschiede in der Lesekompetenz zwischen Frauen und Männern, welche in Hypothese 2 angenommen wurden, identifizieren zu können, wurde ein multipler Gruppenvergleich berechnet. Die durchschnittliche Lesekompetenz von Frauen wurde mit der durchschnittlichen Lesekompetenz von Männern für jeden Faktor verglichen.

Das Modell wurden mit der Software Mplus berechnet (Version 8; Muthén \& Muthén, 2017). 


\subsubsection{Zentrale Befunde}

Die Ergebnisse des Bi-Faktor-Modells zeigten, dass die meisten Items (41 von 68 Items) mit einer substantiellen Ladung auf den Generalfaktor der Lesekompetenz laden. Es kann daher davon ausgegangen werden, dass die Lesekompetenz mit dem Generalfaktor gemessen wurde.

Im Gegensatz dazu konnten die beiden spezifischen Faktoren zur Identifizierung der geschlechtsstereotyp weiblichen und männlichen Textinhalte nicht spezifiziert werden. Für einen überwiegenden Teil der Items konnten keine signifikanten Ladungen auf die angenommenen geschlechtsstereotypen Textkonnotationen festgestellt und keine zusätzliche Varianz über den Generalfaktor hinaus identifiziert werden. Somit deuten die Ergebnisse darauf hin, dass ein eindimensionales Modell zur Messung der Lesekompetenz die vorhandenen Daten am besten erklärt.

Zusammenfassend konnte Hypothese 1 damit nicht bestätigt werden. Anders als angenommen, konnten keine geschlechtsspezifischen Faktoren der Lesekompetenz in geschlechtsstereotypen weiblichen und männlichen Textinhalten identifiziert werden.

Hinsichtlich Hypothese 2, konnte festgestellt werden, dass es keinen signifikanten Unterschied zwischen Männern und Frauen in der Lesekompetenz in den geschlechtsstereotyp männlichen Textinhalten gibt.

In Bezug auf Lesekompetenzunterschiede in den geschlechtsstereotyp weiblichen Textinhalten lag jedoch ein signifikanter Unterschied zwischen Frauen und Männern vor. Dieser Unterschied basiert lediglich auf drei Items mit signifikanten Faktorladungen. Allerdings bezog sich die Annahme auf die gesamten Textinhalte und nicht auf Einzelitems. Daher konnte die angenommene Wechselwirkung von Lesereigenschaften und Textmerkmalen, welche in Hypothese 2 angenommen wurde, nicht bestätigt werden. 


\section{Zusammenfassung der Befunde und Anschlussfragen}

Die Lesekompetenz ist eine essentielle Schlüsselkompetenz für moderne Gesellschaften (Europäische Kommission, 2018; Tippelt, 2018). Jedoch sind für verschiedene Personengruppen Unterschiede in der Lesekompetenz aufgrund von individuellen Merkmalen der Lesenden empirisch zu belegen (u. a. Becker \& McElvany, 2018; Berendes et al., 2018; Nagy et al., 2017). Ein mögliches individuelles Merkmal, welches für die Diskrepanz in der Lesekompetenz zwischen einzelnen Gruppen herangezogen werden kann, stellt das Geschlecht der Lesenden dar. Wie in der theoretischen Ausarbeitung aufgezeigt, ist dabei eine differenzielle Entwicklung von Lesekompetenzunterschieden zwischen Mädchen/Frauen und Jungen/Männer über verschiedene Altersbereiche, beginnend mit dem Ende der Grundschulze it bis ins Erwachsenenalter, zu beobachten (z. B. Berendes et al., 2018; De Fraine et al., 2007; Maehler et al., 2013; McElvany et al., 2017; Nagy et al., 2017; OECD 2019; Solheim \& Lundetræ;2017; Weis, Doroganova et al., 2019). Diese Unterschiede in der Lesekompetenz bei Frauen und Männern können insbesondere anhand verschiedener Determinanten auf Seiten der Lesenden (u. a. Artelt et al., 2007; McElvany \& Becker, 2010; Schiefele et al., 2012) und der Texte (u. a. Lafontaine \& Monseur, 2009; Solheim \& Lundetræ, 2017) erklärt werden.

Um die Gründe für Lesekompetenzunterschiede zwischen Männern und Frauen besser verstehen zu können, wurden für die vorliegende Ausarbeitung zwei Zielsetzungen formuliert: Zum einen wurde für das erste Forschungsziel die Entwicklung von Geschlechtsunterschiede in der Lesekompetenz im jungen Erwachsenenalter fokussiert. Zum anderen wurde für das zweite Forschungsziel die Betrachtung von Determinanten zur Erklärung von Geschlechtsunterschieden in der Lesekompetenz bei Jugendlichen und Erwachsenen genauer untersucht. Auf Basis des Forschungsstands wurden drei zentrale Forschungsfrage abgeleitet. Diese Forschungsfragen wurden jeweils in einzelnen Beiträgen untersucht. Im Folgenden sollen die Ergebnisse der empirischen Untersuchungen miteinander sowie mit den zugrundeliegenden theoretischen Überlegungen verknüpft werden. Damit einhergehend werden Implikationen formuliert und ein Ausblick auf weiterführende Forschungsfragen gegeben. 
Die Forschungsfrage zu Beitrag 1 umfasste eine Beschreibung der Geschlechtsunterschiede in der Lesekompetenz bei jungen Frauen und Männern zwischen 16 und 22 Jahren im Längsschnitt. Es wurde hierbei der Frage nachgegangen, ob die Unterschiede in der Lesekompetenz zwischen jungen Frauen und Männern im Übergang von der Sekundarschulbildung in der Zeit nach der Schule weiterhin vorhanden sind oder sich verringern bzw. ganz verschwinden.

Dabei schloss die erste Forschungsfrage an bereits bekannte Ergebnisse zu Geschlechtsunterschieden in der Lesekompetenz an: Wie bereits in vielen Studien aus dem Altersbereich der Sekundarstufe I konstatiert wurde (z. B. Berendes et al., 2018; Stanat \& Kunter, 2002; Weis, Doroganova et al., 2019), konnten auch in der durchgeführten Studie Lesekompetenzunterschiede in ähnlichem Umfang zwischen jungen Frauen und Männern festgestellt werden. Es zeigte sich, dass junge Frauen in diesem Altersbereich, zum Ende der 9. Klasse mit ca. 16 Jahren, im Durchschnitt eine höhere Lesekompetenz aufweisen als junge Männer. Im jungen Erwachsenenalter von 22 Jahren konnte die durchgeführte Analyse jedoch keinen Unterschied mehr in der Lesekompetenz zwischen Frauen und Männern nachweisen. Dies entspricht den Ergebnissen der PIAAC-Studie, wonach es keine Anhaltspunkte dafür gibt, dass sich die Lesekompetenz zwischen erwachsenen Frauen und Männern (16 - 64 Jahren) unterscheidet (OECD, 2016). Im Vergleich zur QuerschnittAnalyse der PIAAC-Daten konnte die Längsschnitt-Analyse in Beitrag 1 hingegen die Entwicklung von Lesekompetenzunterschieden im Lebensverlauf untersuchen, und darüber hinaus einen Beitrag zur Analyse der Weiterentwicklung der Lesekompetenz bei jungen Erwachsenen nach der Sekundarstufe I leisten. Es ist hervorzuheben, dass vor allem die Lesekompetenz der jungen Männer von 16 bis 22 Jahren linear zu nahm, wodurch der Unterschied in der Lesekompetenz $\mathrm{zu}$ den jungen Frauen ausgeglichen werden konnte. Durch die Analyse konnte gezeigt werden, dass besonders im Alter zwischen 18 und 22 Jahren die jungen Männer ihre Lesekompetenz im Vergleich zu den jungen Frauen nochmals deutlich steigern. Bei den jungen Frauen war im Vergleich in diesem Altersbereich keine Veränderung festzustellen. Die jungen Frauen erreichten im Alter von 18 Jahren ein Plateau der Lesekompetenz, welches bis zum Alter von 22 Jahren konstant blieb. Im Gegensatz zu den Ergebnissen anderer Längsschnitt-Studien mit Schülerinnen und Schülern in einem jüngeren Altersbereich (Klasse 5-8: Berendes et al., 2018; Klasse 9-10: Nagy et al., 2017) deuten die Ergebnisse der vorliegenden Forschungsarbeit auf einen Kompensationseffekt der jungen Männer im Vergleich zu den jungen Frauen hin. 
Zusammenfassend ist folglich für die Beantwortung der ersten Forschungsfrage zu konstatieren, dass die Geschlechtsunterschiede in der Lesekompetenz bei jungen Frauen und Männern zwischen 16 und 22 Jahren abnehmen und im jungen Erwachsenenalter nicht mehr vorhanden sind. Gleichzeitig konnte durch die Analyse gezeigt werden, dass ein differenzieller Verlauf der Lesekompetenzentwicklung für junge Frauen und Männer festzustellen ist.

Deskriptiv kann mit der vorliegenden Studie die Entwicklung der Lesekompetenz von jungen Erwachsenen im Übergang von der Sekundarschulbildung in die Zeit nach der Schule aufgezeigt werden. Über erklärende Mechanismen für dieses Ergebnis kann an dieser Stelle indes nur gemutmaßt werden. Diese Einschränkung der vorliegenden Analyse, ist bedingt durch die Auswertung von Sekundärdaten. In den NEPS-Daten der Startkohorte Klasse 9 (SC4) wurden mögliche Determinanten für die Lesekompetenz teilweise im Querschnitt jedoch nicht im Längsschnitt erfasst. Diese Lücke ist jedoch gleichzeitig ein Ausblick für weitere Forschungsfragen in diesem Altersbereich.

Eine mögliche Erklärung für den differenziellen Verlauf der Lesekompetenz von jungen Frauen und Männern, und insbesondere für den stetigen Zuwachs der Lesekompetenz der jungen Männer im Vergleich zu den jungen Frauen, könnte an einer Änderung der Zielorientierung von Männern und damit einhergehend einer Anpassung der Lesemotivation und des Leseverhaltens im jungen Erwachsenenalter sein. In Abschnitt 4.2 wurde herausgearbeitet, dass die Lesemotivation und das Leseverhalten (Lesemenge, Lesehäufigkeit und Präferenzen für verschiedene Textgenres) wichtige Prädiktoren für die Lesekompetenz sind (z. B. McElvany et al., 2008; Schiefele et al., 2012; Stanat \& Kunter, 2002).

In den Studien von Becker und Kolleginnen und Kollegen (2017; 2018) deuten die Ergebnisse daraufhin, dass es im Übergang von der Schule in die Berufsausbildung und die Universität zu einer Verschiebung der Zielorientierung (Lern- und Leistungsziele) kommt. Hier konnten besonders für Schulabsolventinnen und -absolventen höhere Lernziele als für Schülerinnen und Schüler festgestellt werden (Becker, Pfost, Schiefer \& Artelt, 2017; Becker, Pfost \& Artelt, 2018). Die Lernziele beinhalten unter anderem, neue Fähigkeiten in unbekannten Situationen zu entwickeln und zu meistern, Leistungen zu optimieren und sich selber weiterzuentwickeln (vgl. Senko \& Dawson, 2017; Wirthwein, Sparfeldt, Pinquart, Wegerer \& Steinmayr, 2013). Diese Ergebnisse legen dementsprechend nahe, dass junge Erwachsene vergleichsweise motivierter sind, ihre eigenen Fähigkeiten, Fertigkeiten und 
Kenntnisse nach dem Schulabschluss zu erweitern. Die Zunahme an Lernzielen könnte auf eine bessere Übereinstimmung der eigenen Ziele und Fähigkeiten und der von außen an einen herangetragenen Erwartungen, zum Beispiel in der Ausbildung, hindeuten. Diese bessere Passung und die selbstgewählten Lernmöglichkeiten könnten zu einem Motivationsschub führen und sich so wiederum positiv auf die Kompetenzentwicklung der jungen Erwachsenen auswirken. Daher könnte ein Anstieg in den Lernzielen ebenso zu einer Zunahme in der Lesemotivation, des Leseverhaltens und der Lesemenge führen, da junge Erwachsene möglicherweise nach dem Ende der Schulzeit vermehrt nach ihren eigenen Interessen und Themengebieten lesen. Im Vergleich zu Schülerinnen und Schülern sollten junge Erwachsene mehr selbstgewählte Lesemöglichkeiten haben, die für ihren Alltag von Relevanz sind, was sich als „Engelskreis des Lesens“ positiv auf die Lesemotivation auswirkt und darüber auf die Lesekompetenz.

Da anhand der verwendeten NEPS-Daten für die Studie nicht zu klären ist, ob diese theoretischen Erklärungsmechanismen auch von empirischer Relevanz sind, bieten die Ergebnisse aus Beitrag 1 einen Ausblick auf weitere Forschungsfragen. Die Annahmen einer Veränderung in den Lernzielen und damit einhergehend der Anstieg der Lesemotivation, des Leseverhaltens und der Lesemenge könnte ein Ausblick auf eine zukünftige Forschungsfrage bei jungen Erwachsenen sein, bei der neben der Lesekompetenz die Zielorientierung sowie die Lesemotivation und das -verhalten im Längsschnitt erfasst werden.

Über die deskriptive Beschreibung der Lesekompetenzunterschiede zwischen Männern und Frauen hinausgehend, wurde in Beitrag 2 mit dem Einfluss der Lesepräferenzen eine personenspezifische Determinante der Lesekompetenz in Sachtexten und literarischen Texten genauer betrachtet. Mit der zweiten Forschungsfrage sollten zum einen Unterschiede in der textsortenspezifischen Lesekompetenz von Frauen und Männern untersucht werden und zum anderen der Einfluss der Lesepräferenzen auf die Lesekompetenz. Die Analyse aus Beitrag 2 konnte insbesondere im Bereich der Lesekompetenzuntersuchung von Erwachsenen sowie zum Einfluss eines Merkmales der Lesenden (Lesepräferenzen) auf die Lesekompetenz einen Forschungsbeitrag leisten.

Wiederholt wurde bereits aufgezeigt, dass es bislang wenige Befunde zur Lesekompetenz von Erwachsenen und speziell der textsortenspezifischen Lesekompetenz in Sachtexten und literarischen Texten gibt. Bezogen auf die allgemeine Lesekompetenz von Erwachsenen, 
sind jedoch vor allem die Ergebnisse der PIAAC-Studie (OECD, 2016) sowie die Ergebnisse aus Beitrag $1 \mathrm{zu}$ nennen. In PIAAC wurde allerdings nur die Textsorte der Sachtexte zur Messung der Lesekompetenz von Erwachsenen herangezogen (Solheim \& Lundetræ, 2017). Durch die Hinzunahme der Messung der Lesekompetenz in literarischen Texten können differenzierte Aussagen für Frauen und Männer auf der Ebene der Textsorten getroffen werden. Deskriptiv konnte in Beitrag 2 zwischen Frauen und Männern (19 - 71 Jahren) kein Unterschied in der Lesekompetenz, weder in Sachtexten noch in literarischen Texten, festgestellt werden. Dieses Ergebnis deckt sich mit den bisherigen Befunden aus der PIAACStudie und dem Ergebnis aus Beitrag 1. Mittels Hinzunahme der Lesepräferenzen zur Unterhaltung (Krimis, Science-Fiction, Romane und Thriller oder Horrorbücher) und Informationsaufnahme (Tages- und Wochenzeitungen, Sachbücher, Biografien), konnte im Mediationsmodell in Beitrag 2 bei Männern ein leichter Vorteil in der Lesekompetenz in Sachtexten im Vergleich zu Frauen beobachtet werden. Ferner kann insgesamt anhand des zweiten Beitrags festgestellt werden, dass die Lesepräferenz in der Freizeit, als motivationa 1behaviorale Einstellung zum Lesen, einen positiven Einfluss auf die Lesekompetenz hat. Dieses Ergebnis aus dem höheren Altersbereich der Lesenden deckt sich mit den vielfältigen Befunden aus dem Kindes- und Jugendalter. Jedoch ist anhand der divergenten Ergebnisse aus Beitrag $2 \mathrm{zu}$ erkennen, dass die Bedeutsamkeit für die Lesekompetenz deutlich vom informierenden Lesen ausgeht und, dass das Lesen zur Unterhaltung weniger relevant für die Lesekompetenz von Erwachsenen ist. Dieser Befund weicht von Ergebnissen aus dem Kinders- und Jungendalter $a b$, in denen das Lesen von narrativen Texten bedeutsamer für die Entwicklung der Lesekompetenz ist als das Lesen von expositorischen Texten (z. B. Mar \& Rain, 2015; Pfost et al., 2013). Eine mögliche Erklärung für dieses Ergebnis könnte mit den Alltagserfahrungen und speziell mit dem beruflichen Alltag von erwachsenen Personen zusammenhängen. In der Studie von Locher und Pfost (2019) konnte bei einer quantifizierten Erfassung der Lesemenge (wie viel Zeit täglich für das Lesen verwendet wird) dargestellt werden, dass der Zusammenhang von gelesener Zeit auf die Lesekompetenz im Freizeitlesen bei Erwachsenen (im Durchschnitt 50 Jahre $r=.12$ ) geringer ist, als dies bei Kindern (im Durchschnitt 11 Jahre $r=.30$ ) und Jugendlichen (im Durchschnitt 15 Jahre $r=.29$ ) der Fall ist. Demgegenüber konnte herausgestellt werden, dass bei den erwachsenen Personen das Lesen für die Arbeit einen bedeutsameren Einfluss auf die Lesekompetenz $(r=.28)$ hat als das Freizeitleseverhalten. Locher und Pfost (2019) stellten fest, dass für Erwachsene die Relevanz des Lesens aufgrund des Berufs steigt und 
gleichzeitig mehr Zeit für berufliches Lesen aufgewendet wird. Auch wenn in Beitrag 2 explizit nach dem Lesen in der Freizeit gefragt wurde, ist nicht auszuschließen, dass der Übergang vom Lesen in der Freizeit oder den Beruf fließend und möglicherweise nicht für alle teilnehmenden Personen kategorisch zu trennen ist. Die Ergebnisse von Beitrag 2 könnten daher in Verbindung mit dem Befund von Locher und Pfost (2019) ein Ausblick für weitere Forschung zum Leseverhalten von Erwachsenen sein. Durch eine qualifizierende Abfrage des Lesematerials (was wird gelesen) für den Arbeitskontext könnte nochmals stärker differenziert und möglicherweise hinsichtlich des beruflichen Lesematerials Präferenzen und Schwierigkeitsgrade im Textmaterial herausgearbeitet werden. In der vorliegenden NEPS-Entwicklungsstudie, welche für Beitrag 2 verwendet wurde, fand keine Erfassung der beruflichen Tätigkeiten oder des beruflichen Leseverhaltens der Teilnehmenden statt. Somit wäre für zukünftige Forschung bei der Erfassung des Leseverhaltens von Erwachsenen nicht nur das Freizeitleseverhalten, sondern ebenso das berufliche Lesen zu berücksichtigen, um deren Auswirkungen auf die Lesekompetenz differenzierter betrachten zu können. Rexing, Keimes und Ziegler (2015) konnten zudem in einer qualitativen Umfrage unter Erwachsenen feststellen, dass die Relevanz des Lesens im Beruf vom Stellenprofil abgängig ist. Aus diesem Grund wäre es in zukünftigen Studien gewinnbringend nach den beruflichen Positionen und dem damit verbundenen Leseaufwand $\mathrm{zu}$ differenzieren.

Beitrag 2 konnte zwar einen ersten Hinweis für einen „Engelskreis des Lesens“ bei Erwachsenen geben, jedoch kann durch die Untersuchung im Querschnitt nichts zu den kausalen Zusammenhängen der Lesepräferenz auf die textsortenspezifische Lesekompetenz gesagt werden. Es wäre durchaus denkbar, dass Personen mit einer höheren Lesekompetenz häufiger in ihrer Freizeit lesen und ebenso eine vielfältigere und anspruchsvollere Lektüre wählen. Diese Annahmen konnte mit den Daten einer NEPS-Entwicklungsstudie aus Beitrag 2 nicht untersucht werden. Um kausale Rückschlüsse auf den „Engelskreis des Lesens“ bei erwachsenen Lesenden ziehen zu können, würde es sowohl eine Erfassung der Lesepräferenzen als auch der textsortenspezifischen Lesekompetenz im Längsschnitt benötigen.

Hinsichtlich der Geschlechtsunterschiede in den Lesepräferenzen konnte Beitrag 2 ebenso wie vorangegangene Studien bestätigen, dass Frauen und Männer in ihrer Freizeit zu einem unterschiedlichen Zweck lesen (z. B. Charlton et al., 2004; Schreier \& Odağ, 2004). Frauen lesen im Allgemeinen häufiger, um sich unterhalten zu lassen (Krimis, Science- 
Fiction, Romane und Thriller oder Horrorbücher) und Männer lesen in ihrer Freizeit häufiger, um Informationen $\mathrm{zu}$ erhalten (Tages- und Wochenzeitungen, Sachbücher, Biografien). In Beitrag 2 wurde über die Abfrage eines Genres auf dessen Lesezweck (zur Unterhaltung oder um Informationen zu erhalten) geschlossen. In zukünftigen Studien könnte erfasst werden, ob das jeweilige Genre wirklich dem vermuteten Lesezweck entspricht. So wurde in der vorliegenden Studie angenommen, dass zum Beispiel Sachbücher oder Biografien der Informationsaufnahme dienen. Im Gegensatz dazu ist es durchaus denkbar, dass Leserinnen oder Leser eine Biografie gleichermaßen zur Unterhaltung lesen. Ebenso könnte ein Sachbuch aus beruflichen Gründen gelesen werden. Infolgedessen könnte eine qualifizierende Erfassung des Gelesenen noch nach weiteren Facetten differenziert werden, um ein umfangreicheres Bild des Leseverhaltens bei Erwachsenen zu erhalten.

Im letzten Beitrag der vorliegenden Arbeit, wird ebenso wie in Beitrag 2, eine weitere Determinante der Lesekompetenz detailliert für Männer und Frauen untersucht. Der Fokus zur Beantwortung der dritten Forschungsfrage lag dabei auf dem Text als erklärender Faktor für Lesekompetenzunterschiede. In Beitrag 3 sollte über geschlechtsstereotype Textinhalte, Unterschiede in der Lesekompetenz von Frauen und Männern identifiziert werden.

Wie in der Ausführung zur sozialen Umwelt in Punkt 4.1 dargestellt, sind nach wie vor sozial geteilte Annahmen darüber vorhanden, was typisch weibliche und männliche Eigenschaften und Verhaltensweisen darstellen (sogenannte Geschlechtsstereotype). Über Sprache werden Stereotype vermittelt (u. a. Garnham et al. 2016), beispielsweise über geschlechtsstereotype Textinhalte, wodurch Ungleichheiten zwischen Frauen und Männern sprachlich aufrechterhalten werden. Vorhandene Geschlechtsstereotype haben einen Einfluss auf das individuelle Interesse und die Motivation, sich mit einem bestimmten Thema zu beschäftigen, darüber Informationen zu erhalten sowie Wissen zu erweitern (Möller \& Schiefele, 2004). Aufgrund dessen wurde für Beitrag 3 angenommen, dass Geschlechtsstereotype durch Textinhalte aktiviert werden und mit Vorwissen aus unterschiedlichen Erfahrungs- und Inhaltsbereichen sowie unterschiedlichen Interessen von Frauen und Männern zu verschiedenen Themen verknüpft werden (z. B. Bildung, Beruf, Freizeitaktivitäten; Baron et al., 2001; Christin, 2012; Lagaert et al., 2017; Nosek et al., 2002). Dies sollte die Motivation zum Lesen von Texten aus vertrauten Inhaltsbereichen bei Frauen und Männern erhöhen und sich darüber auf deren Lesekompetenz auswirken, in 
Abhängigkeit davon, ob der Inhalt eines Textes eher stereotyp weiblich oder eher stereotyp männlich konnotiert ist.

Die Ergebnisse der durchgeführten Studie konnten die Annahmen nicht bestätigen, dass geschlechtsstereotype Textinhalte einen Einfluss auf die Lesekompetenz von Männern und Frauen haben. In der Dimensionsanalyse konnten auf der Ebene des Textmaterials die geschlechtsspezifischen Faktoren der Lesekompetenz in geschlechtsstereotypen weiblichen und männlichen Textinhalten nicht identifiziert werden.

Damit entsprechen die Ergebnisse aus Beitrag 3 nicht den Ergebnissen anderer Studien. In den Studien von McElvany und Kolleginnen (2016) sowie El-Khechen und Kolleginnen (2017) wurde beispielsweise festgestellt, dass Kinder im Grundschulalter häufiger Wörter kannten, welche mit Stereotypen übereinstimmen, die mit ihrem eigenen Geschlecht assoziiert sind, als Wörter, die mit der anderen Geschlechtsgruppe assoziiert sind. Basierend auf diesem Ergebnis, wurde in der durchgeführten Studie aus Beitrag 3 für das Textmaterial ebenso angenommen, dass erwachsene Lesende geschlechtsstereotypen Textinhalte auf das eigene Geschlecht beziehen. Dies scheint allerdings auf die Altersgruppe von Erwachsenen (19 - 65 Jahren) nicht zuzutreffen. Möglicherweise können Erwachsene gerade durch vielfältiges Vorwissen und Erfahrungen aus verschiedenen Themengebieten stärker zwischen sich selbst und dessen, wie Frauen und Männer typischerweise sein sollten, differenzieren. Daher wäre es von Interesse, zukünftig eine ähnliche Studie mit Kindern und Jugendlichen durchzuführen, um festzustellen, ob diese Annahme der Differenzierung von Geschlechtsstereotypen und dem eigenen Verhalten und Interessen wirklich spezifisch auf Erwachse zutrifft und jüngere Teilnehmende vielleicht bei der bewussten Wahrnehmung von Geschlechtsstereotypen weniger sensitiv sind. Zum anderen könnte das Ergebnis auf die a priori kategorisierten Texte und Inhalte im Lesekompetenztest zurückgeführt werden. Der für die durchgeführte Studie verwendete NEPS-Lesekompetenztest ist so konzipiert, die Lesekompetenz verschiedener Altersgruppen zu erfassen (siehe NEPS-Rahmenkonzeption zur Erfassung der Lesekompetenz: Gehrer et al., 2013). Dabei ist der Test so entwickelt, dass durch die gestellten Aufgaben möglichst keine Subgruppen bevorzugt oder benachteiligt werden, was durch DIF-Analysen der Items in der Testentwicklung geprüft wird. Es könnte sein, dass der erwartete Effekt eintritt, wenn Leserinnen und Leser mit thematisch komplexeren Texten konfrontiert werden, welche stärker die Lesemotivation herausfordern. Diese Annahme könnte zukünftig in einer experimentelleren Studie geprüft werden, in der eigens Texte mit deutlicheren Geschlechtsstereotypen verwendet und manipuliert werden. 
Für die Erfassung der Lesekompetenz von Männern und Frauen ist unter dem Aspekt der Testfairness das erfreuliche Ergebnis festzuhalten, dass neben der Dimension der Lesekompetenz keine weiteren spezifischen Faktoren identifiziert werden konnten. Anders als die Ergebnisse zum Format der Texte (kontinuierliche vs. nicht-kontinuierliche Texte; siehe u. a. Stanat \& Kunter, 2002) oder den Item-Formaten (offene vs. geschlossene Aufgaben; siehe u. a. Lafontaine \& Monseur, 2009), kann für die Entwicklung eines Lesekompetenztests die Facette der geschlechtsstereotypen Textinhalte demnach vernachlässigt werden.

Darüber hinaus lassen sich keine Unterschiede in den geschlechtsstereotypen Faktoren der Lesekompetenz zwischen Frauen und Männern nachweisen. Unabhängig von möglichen Geschlechtsstereotypen in den Textinhalten zeigten Männer und Frauen eine vergleichbare Lesekompetenz. Somit stimmen die Ergebnisse von Beitrag 3 mit den bisherigen anderen Beiträgen und der PIAAC-Studie (OECD, 2016) überein.

Zusammenfassend ist für Beitrag 3 festzuhalten, dass keine signifikanten Unterschiede in den geschlechtsstereotypen Faktoren sowie der Lesekompetenz zwischen Frauen und Männern festgestellt werden konnten. Jedoch wäre eine weitere Anschlussfragestellung die hinsichtlich der geschlechtsstereotypen Textinhalten untersucht werden könnte, inwiefern ein stereotyper Inhalt eine Auswirkung auf das Bearbeitungsverhalten der Lesenden hat. Durch eine computerbasierte Erfassung der Lesekompetenz ist die Möglichkeit vorhanden, auf Hintergrundinformationen, welche vom Computer während der Testung erfasst werden, zuzugreifen. Mittels der Erfassung von Hintergrundinformationen ist es möglich, Lesezeiten der Texte, Bearbeitungszeiten und -verhalten zu den Aufgaben zu sammeln. Dies bietet einen Einblick in das individuelle Bearbeitungs- und Lösungsverhalten eines Kompetenztests und ermöglicht dadurch weitere wissenschaftliche Fragestellungen (Goldhammer et al., 2019; Kröhne \& Martens, 2011). Über die Auswertung von computerbasierten Hintergrundinformationen könnten potenzielle Unterschiede zwischen Frauen und Männern in ihrem Bearbeitungsverhalten der Texte festgestellt werden. Möglicherweise könnten das Vorhandensein eines Stereotyps im Textinhalt einen Einfluss auf die Bearbeitungszeit haben und diese für die jeweils andere Geschlechtsgruppe verlangsamen. 


\section{Gesamtschau und Ausblick}

Im Rückblick auf die Einführung dieser Arbeit, verfolgte die vorliegende Arbeit zwei Ziele: zum einen die Untersuchung der Entwicklung von Geschlechtsunterschieden in der Lesekompetenz, im Übergang aus dem institutionellen Lernkontext der Schule heraus. Zum anderen wurde der Einfluss von personen- und textsortenspezifische Determinanten auf die Lesekompetenz von Frauen und Männern genauer betrachtet.

Im Folgenden wird über die Ergebnisse der drei Beiträge hinaus, eine Gesamtschau zur Entwicklung der Lesekompetenz über die Lebensspanne sowie deren Determinanten dargestellt. Des Weiteren wird ein Ausblick auf weiterführende Aspekte hinsichtlich der Lesekompetenz von erwachsenen Frauen und Männern gegeben.

Im Vergleich zur Befundlage über die Lesekompetenz von Kindern und Jugendlichen sowohl hinsichtlich deren Entwicklung als auch zu deren Einflussfaktoren, ist in Bezug auf die Entwicklung der Lesekompetenz nach dem Ende der Schulzeit sowie darüber hinaus weit weniger bekannt. Insofern konnte die vorliegende Arbeit an bisherige Beiträge aus dem Altersbereich von Kindern und Jugendlichen anknüpfen. Jedoch sind noch weitere Anschluss-Studien nötig, um die Lesekompetenz im Allgemeinen, aber ebenso in Bezug auf Subgruppen, wie Männer und Frauen, über die gesamte Lebenspanne hinweg, beschreiben zu können. Erstrebenswert wäre hierzu eine Datenlage und Auswertung zur Lesekompetenzentwicklung im Längsschnitt, beginnend mit dem Ende der Schulzeit bis ins hohe Erwachsenenalter.

Allerdings ist bei der Fragestellung der Lesekompetenzentwicklung von Erwachsenen, deren große Heterogenität hinsichtlich der Determinanten auf die Lesekompetenz, aber auch der Kompetenz in der Gesamtgruppe nicht außer Acht zu lassen. Im Erwachsenenalter gibt es eine Vielzahl an möglichen Bildungskarrieren, Berufsverläufen, Lebenswegen und entscheidungen, die einen Einfluss auf die Kompetenzverläufe nehmen können. Diese Heterogenität der möglichen Einflussfaktoren auf die Lesekompetenz innerhalb der Gesamtgruppe der Erwachsenenbevölkerung ist eine mögliche Erklärung für abweichende Ergebnisse im Vergleich zu Kindern und Jugendlichen.

Auf Grund der großen Verschiedenartigkeit von Erwachsenen in der Gesellschaft sollte daher zukünftig anvisiert werden, nicht nur ein erklärendes Merkmal für die Entwicklung von Kompetenzverläufen zu betrachten. Eine mögliche Herangehensweise wäre das 
Heranziehen des Konzeptes der Intersektionalität aus der Diskriminierungsforschung. Dieser integrative Ansatz geht davon aus, dass es eine Verschränkung von verschiedenen Dimensionen der Ungleichheit und Differenz innerhalb einer Person gibt. Aus einem Konglomerat an gesellschaftlich konstruierten Differenz-Kategorien, wie zum Beispiel das Geschlecht, die Ethnizität oder das Alter einer Person, entstehen Privilegien sowie Diskriminierungen von Individuen (u. a. Bronner \& Paulus, 2017). Einen ähnlichen Ansatz könnte man zur Erforschung der Lesekompetenzentwicklung von Erwachsenen in Betracht ziehen. Wie bereits bei Kindern und Jugendlichen bekannt ist, hat der soziale Hintergrund in Form der Bildung der Eltern, deren Einkommen sowie der Migrationsstatus, einen Einfluss auf die Lesekompetenz und deren Entwicklung (Becker et al., 2010; Berendes et al., 2018; Nagy et al., 2017; Retelsdorf et al., 2011). Ähnliche Einflüsse könnten im Lesekompetenzverlauf von Erwachsenen, neben dem Geschlecht einer Person, weiterhin von Relevanz sein. Erste Hinweise auf diese Annahme können aus der Studie von Wicht, Rammstedt und Lechner (2020) entnommen werden. So konnte mit den NEPS-Daten für 4,359 Erwachsene zwischen 24-69 Jahren festgestellt werden, dass ferner soziodemographische Einflussfaktoren, insbesondere Schulbildung, Alter sowie gelernte Erstsprache in der Kindheit, einen entscheidenden Einfluss auf die Lesekompetenz haben. Die Autorinnen und Autoren schlussfolgern einen Matthäuseffekt, der sich statusbedingt im Laufe des Lebens weiter vergrößert (Wicht et al., 2020). Daher wäre es für die weitere Forschung zur Lesekompetenzentwicklung bei Erwachsenen wünschenswert, neben dem Geschlecht weitere Faktoren, wie möglicherweise Alter, Bildungsabschlüsse, beruflicher Stand, Teilnahme an Fort- und Weiterbildungen, Migrationshintergrund, gesprochene Sprache im Haushalt oder Anzahl an Kindern genauer zu untersuchen, um Stärken und Schwächen von Personen in deren Lesekompetenz genauer abbilden zu können.

Anknüpfend an die getroffenen Annahmen, dass die Lesekompetenz eine Schlüsselkompetenz ist, welche aktiv und interaktiv dazu genutzt wird, um verschiedene Anforderungen des Alltags, der Arbeit und des sozialen Lebens zu bewältigen (u. a. Tippelt, 2018) sowie kontinuierlich über die Lebensspanne hinweg weiterentwickelt wird (u. a. Alexander, 2005), stellt sich daraus die Anschlussfrage, was Entwicklung der Lesekompetenz bei erwachsenen Personen bedeutet. Wie die Ergebnisse aus dem Kindesund Jugendalter zeigen, steigt im Allgemeinen für Schülerinnen und Schüler die Lesekompetenz mit dem Eintritt in die Schule stetig an (z. B. Philipp, 2011; Retelsdorf et 
al., 2011). Ferner konnte in Beitrag 1 ein Zuwachs der Lesekompetenz von jungen Erwachsenen insgesamt, und für Frauen und Männer getrennt nachgewiesen werden. Jedoch stellt sich daran anschließend die Frage, ob von einem weiteren bedeutsamen Anstieg der Lesekompetenz nach dem Verlassen der Schule auszugehen ist oder die Lesekompetenzentwicklung im Erwachsenenalter stagniert bzw. sie mit zunehmendem Alter wieder abnimmt. An dieser Stelle kann zum jetzigen Zeitpunkt nur spekuliert werden, da die Ergebnislage bisher sehr eingeschränkt ist. Anknüpfend an die ausgeführten Überlegungen, wäre für die Beantwortung dieser Frage, wie im vorangehenden Kapitel schon mehrfach erwähnt, eine Längsschnitt-Datenauswertung der Lesekompetenz über eine längere Lebensspanne für weitere gesicherte Aussagen nötig. Ein erster Hinweis auf den weiteren Verlauf der Lesekompetenz bei Erwachsenen liefert die Studie von Wicht und Kollegin sowie Kollege (2020), in welcher im Verlauf von sechs Jahren gezeigt werden konnte, dass sich die Lesekompetenz der Erwachsenen zwischen 24 - 69 Jahren im Mittel nur um 0.01 Logits verändert hat. Indes liegt eine Streuung von 1.03 Logits vor, was wiederum für eine Heterogenität der Lesekompetenz innerhalb der Stichprobe von Erwachsenen spricht.

Wie in Beitrag 1 als möglicher erklärender Mechanismus für die dargestellten Ergebnisse genannt, scheinen relevante Lebensereignisse, wie etwa das Verlassen der Schule und der Beginn der beruflichen und universitären Ausbildung, eine Veränderung in der Lesekompetenz herbeizuführen. Möglicherweise verändert sich die Lesekompetenz bei Erwachsenen weniger stark als dies in der Schule noch der Fall ist (Wicht et al, 2020). Allerdings könnten relevante Lebensereignisse bei Erwachsenen, wie zum Beispiel eine berufliche Veränderung, Weiterbildungen oder die Erziehung von Kindern, ebenso einen Einfluss auf die Lesekompetenz haben. In Studien zum gemeinsamen Lesen in der Familie zeigt sich, das Vorlesen für Kinder einen positiven Effekt auf deren sprachliche Entwicklung hat (z. B. Mol, Bus, de Jong \& Smeets, 2008; Niklas, 2015; Weinert \& Ebert, 2013). Im Umkehrschluss könnte man ebenso mutmaßen, dass Vorlesen einen positiven Einfluss auf die Lesekompetenz der Eltern hat und so wiederum zu einem Anstieg der Lesekompetenz der Erwachsenen in dieser Phase des Lebens führen kann.

Des Weiteren wäre denkbar, dass in einem höheren Alter durch nachlassende kognitive Funktionen, der Prozess des Lesens verlangsamt bzw. eingeschränkt wird und dies wiederum einen negativen Einfluss auf die Lesekompetenz bei Seniorinnen und Senioren hat. Wie die allgemeine Verlangsamungs-Hypothese (,general slowing hypothesis“) postuliert, kommt es dabei im Verlauf des Älterwerdens zu einer Abnahme der 
Verarbeitungsgeschwindigkeit von Informationen (Cerella, Poon \& Williams, 1980). Dies könnte sich wiederum negativ auf den Leseprozess und die Lesekompetenz auswirken. Als Konsequenz aus dieser möglichen Abnahme der Lesekompetenz könnten ältere Menschen daher in ihrem Altag und der sozialen sowie gesellschaftlichen Teilhabe beeinträchtigt sein. Einen ersten Hinweis auf diese Annahme liefert gleichfalls die Studie von Wicht und Kollegin sowie Kollege (2020), in der gezeigt werden konnte, dass ein höheres Alter der Teilnehmenden eine Abnahme in der Lesekompetenz zur Folge hatte. Für eine abschließende Schlussfolgerung wäre zu differenzieren, ob es sich tatsächlich um einen Effekt auf Basis des Alters der Teilnehmenden handelt oder ob möglicherweise in der Stichprobe von 24 - 69-jährigen ein Kohorteneffekt vorliegt.

Die hier angesprochene größere Heterogenität unter den Erwachsenen, sowohl in Bezug auf die Determinanten der Lesekompetenz als auch die Lesekompetenz selbst verdeutlichen, dass sich eine Leseförderung bei Erwachsenen weniger einfach gestaltet als dies bei Kindern und Jugendlichen innerhalb von Bildungseinrichtungen der Fall ist. Möglicherweise gestaltet sich die Förderung der Lesekompetenz von Erwachsenen weniger strukturiert und eher implizit durch Fort- und Weiterbildungen sowie kulturelle Einrichtungen wie zum Beispiel durch vielfältige Angebote von Bibliotheken. Diese können exemplarisch als wichtige Anlaufpunkte angeführt werden, welche Literatur in diversen Darbietungsformen (z. B. gedruckte Bücher, Zeitschriften, E-Books) zur Verfügung stellen und zugänglich machen. Viele Bibliotheken bieten darüber hinaus umfassende kulturelle Angebote an, zum Beispiel Autorenlesungen oder Lesekreise, um das Lesen zu fördern und Interesse an Themen zu wecken. Über solche Angebote könnte man sich wieder den „Engelskreis des Lesens“ zu Nutze machen und Leseanlässe sowie -interesse zu schaffen, um darüber die Lesekompetenz zu fördern. Dies sollte exemplarisch verdeutlichen, dass die explizite Förderung der Lesekompetenz von Erwachsenen, im Vergleich zu der von Kindern und Jugendlichen, noch stärker fokussiert werden könnte.

Die Befunde der drei Beiträge zeigen, dass es im Erwachsenenalter keine Geschlechtsunterschiede in der Lesekompetenz zwischen Frauen und Männern gibt. Dieses positive Resultat sollte allerdings nicht als Argument für eine Vernachlässigung der Förderung der Lesekompetenz genutzt werden. Betreffend die Geschlechtsunterschiede in der Lesekompetenz im Kindes- und Jugendalter, sollte speziell hier weiterhin ein Augenmerk auf die Förderung der Lesekompetenz von Jungen und Mädchen gelegt werden. 
Wie gezeigt hat die soziale Umwelt einen bedeutenden Einfluss auf die Wahrnehmung des Lesens. So gilt das Lesen eher als stereotyp weiblicher Gegenstandsbereich, an dem mehr Frauen als Männer interessiert sind und Mädchen auch als begabter eingeschätzt werden (u. a. Heyder \& Kessels, 2013; Steffens \& Jelenec, 2011; Watson et al., 2010). Wie mit dem Erwartungs-Wert-Modell dargestellt (siehe u. a. Möller \& Schiefele, 2004), haben Geschlechtsstereotype einen Einfluss auf die Lesemotivation sowie das lesebezogene Selbstkonzept. Generell wäre es wünschenswert, dieser Verbindung entgegenzuwirken, sodass die soziale Umwelt die Tätigkeit des Lesens weniger mit Mädchen/Frauen verbindet. Jedoch könnte sich gerade für die Förderung der Lesekompetenz ein Geschlechtsstereotyp hinsichtlich der Nutzung von Medien zu eigen gemacht werden. Wie Befunde zeigen korreliert das Interesse für das Lesen bei Jungen/Männern stärker mit dem Medium als dies bei Mädchen/Frauen der Fall ist (z. B. Diedrich et al., 2019; Duncan, McGeown, Griffiths, Stothard \& Dobai, 2016; McElvany \& Schwabe, 2019).

In dieser Arbeit wurde bisher die mögliche Darbietungsform des Lesematerials in gedrucktem sowie digitalem Format nicht detailliert diskutiert und in die Fragestellung zu Geschlechtsunterschieden in der Lesekompetenz einbezogen. Das digitale Lesen ist jedoch mittlerweile nicht mehr aus dem Leseverhalten und -materialien wegzudenken. Gleichzeitig könnte die Darbietungsform des Lesematerials auch zur Erklärung von Geschlechtsunterschieden in der Lesekompetenz und deren Förderung herangezogen werden.

Zukünftig könnte hier in weiteren Untersuchungen eine genauere Spezifizierung vorgenommen werden. Zum einen könnten bestimmte Genres, wie möglicherweise Zeitungen und Zeitschriften, häufiger digital gelesen werden als dies unter Umständen bei Romanen der Fall ist. Zum anderen könnten indessen innerhalb des Genres und der Darbietungsform Geschlechtsunterschiede vorhanden sein. Ebenso sollte die Nutzung und das Lesen von Chats in Messenger-Diensten sowie die Lesegewohnheiten in sozialen Medien in Lesekompetenzuntersuchungen stärker berücksichtigt werden. In den PISADaten von 2018 zeigt sich in den Ergebnissen die große Relevanz des Online-Lesens für die 15-Jährigen, sowohl für Mädchen als auch für Jungen. Am häufigsten lesen die Jugendlichen Chats aus Text-Nachrichten-Diensten. Jedoch sind Online-Nachrichten, -Themen und Informationen ähnlich relevant für die Teilnehmenden (Diedrich et al,, 2019). Der Zusammenhang dieser spezifischen Darstellungsform des Online-Lesens mit der 
Lesekompetenz sollte für Jugendliche, aber auch für Erwachsene zukünftig noch differenzierter untersucht werden, um spezifische Förderangebote abzuleiten zu können.

Die Relevanz und Zunahme des digitalen Lesens konnten Duncan und Kolleginnen (2016) bestätigen. In ihrer Untersuchung unter 15-jährigen Schülerinnen und Schülern gaben die Jugendlichen an, mehr Zeit mit dem Lesen von digitalen als gedruckten Texten zu verbringen. Hinsichtlich der Präferenzen konnte dennoch ausführlich dargestellt werden, dass Jungen den Computer häufiger für PC-Spiele verwenden, während Mädchen im Durchschnitt mehr den Computer für Text- und E-Mail-Nachrichten, Webseiten und soziale Netzwerke nutzen (Duncan et al., 2016). Gleichzeitig konnten McElvany und Schwabe (2019) in einer Untersuchung bei Viertklässlern feststellen, dass Jungen im Vergleich zu Mädchen eine höhere digitale Lesemotivation (z. B. „Ich arbeite gerne an digitalen Geräten“) und ein höheres digitales Selbstkonzept (z. B. „Die Arbeit an digitalen Geräten fällt mir leicht") haben. Somit ist für die Förderung der Lesekompetenz von Jungen festzuhalten, dass diese positive Einstellung zu digitalen Geräten dafür genutzt werden könnte, diese nicht nur als Plattformen für PC-Spiele zu sehen, sondern diese gleichzeitig für das digitale Lesen zu nutzen.

Nicht nur für die Lesenden ist die Digitalisierung ein Zugewinn, sondern auch für die Messung und Testkonstruktion der Lesekompetenz, bietet die digitale Erfassung einen Mehrwert und repräsentiert dadurch die realen Lesebedingungen der Teilnehmenden. Ein Vorteil der digitalen Lesekompetenztestung ist die Verwendung von interaktiven Aufgabenund Antwortformaten (Beispiele für neue Aufgaben- und Antwortformaten in PISA 2018 bei Becker-Mrotzek et al., 2019; in NEPS bei Rohm et al., 2019).

Jedoch ist zu diskutieren, ob durch den Wechsel von Papierbasierter-Testung (PBA) zu Computerbasierter-Testung (CBA) und den damit verbundenen Neuerungen in den Textund Aufgabenformaten ein anderes Verständnis von Lesekompetenz gemessen wird. Möglicherweise kommt zur Lesekompetenz noch der Umgang mit digitalen Herausforderungen hinzu, wie das Navigieren oder das Verstehen animierter Grafiken. Dies würde eher einem Konzept von digital reading entsprechen (Naumann \& Sälzer, 2017). Um allerdings von einem ähnlichen Modus auszugehen und damit den Prozess der Lesekompetenz so ähnlich wie möglich zu messen, sowohl in PBA als auch in CBA, sollten sich die Texte und Aufgaben in digitaler Form möglichst wenig von den Texten und Aufgaben in gedruckter Form unterscheiden (reading digitally; Naumann \& Sälzer, 2017). 
Daher werden bei der Umstellung von PBA auf CBA-Testungen immer wieder methodische Herausforderungen sowie die Frage nach der Konstruktvalidität diskutiert. Durch eine Umstellung des Testformats kann es zu sogenannten Modus-Effekten kommen, welche sich auf die Schwierigkeit und die Trennschärfe von einzelnen Aufgaben ebenso wie auf die Interpretation von Testwerten auswirken kann (Bürger, Kröhne \& Goldhammer, 2016; Kröhne, Bürger, Hahnel \& Goldhammer, 2019; Kröhne \& Martens, 2011).

Hinsichtlich der Lesekompetenz von Jungen und Mädchen wird sich im Zusammenhang mit der digitalen Erfassung der Lesekompetenz wiederkehrend mit Modus-Effekten auseinandergesetzt. Begründet wird dieser Modus-Effekt mit der oben aufgeführten stärkeren Affinität der Jungen für den Umgang mit digitalen Aktivitäten (z. B. Diedrich et al., 2019; Duncan et al., 2016; McElvany \& Schwabe, 2019). In einer Studie mit Daten aus PISA 2012 konnten Kröhne, Hahnel und Goldhammer (2019) zeigen, dass der Wechsel von einer PBA-zu CBA-Testung Kompetenzunterschiede zwischen Jungen und Mädchen nicht verstärken. Die gefundenen Unterschiede in der Lesekompetenz zwischen den 15-jährigen Mädchen und Jungen können nicht auf den Modus zurückgeführt werden. Indessen konnte die Studie zeigen, dass der Wechsel des Modus, die Schwierigkeit der Aufgaben beeinflusst und diese in der CBA-Testung schwieriger sind als in der PBA-Testung.

Weitere Forschung zum digitalen Lesen sowie zu Modus-Effekten steht noch aus. Insbesondere abgeleitet aus den Ergebnissen, ob Lesen und reading digitally möglicherweise doch verschiedene Konstrukte sind (Kröhne, Bürger et al., 2019; Naumann \& Sälzer, 2017; Robitzsch et al., 2017), sollte dies für LesekompetenzErhebungen weiterhin genauer untersucht werden. Des Weiteren ist auch zur Messung von Modus-Effekten bei Erwachsenen wenig bekannt. So könnte ein Wechsel des Testformats bei erwachsenen Teilnehmenden stärker mit dem Alter sowie der Affinität zu digitalen Geräten zusammenhängen. Ältere Studienteilnehmende könnten weniger an das Lesen am Bildschirm und die interaktiven Aufgabenformate gewöhnt sein als dies bei jüngeren Teilnehmenden der Fall ist.

Abschließend soll auf die Höhe der Geschlechtsunterschiede in der Lesekompetenz eingegangen und der Umgang damit diskutiert werden. Es wurde in der Ergebnisdarstellung ausführlich aufgezeigt, dass augenscheinlich beim Vergleich der drei Large-Scale Assessments PIRLS, PISA und PIAAC der Eindruck entsteht, dass sich die Geschlechtsunterschiede zwischen der Erfassung der Lesekompetenz von PIRLS (4. Klasse) 
und PISA (15-jährige Schülerinnen und Schüler) vergrößern (vgl. Mullis etal., 2017; OECD, 2019; Solheim \& Lundetræ, 2017). Im weiteren Verlauf scheint sich die Schere zwischen Frauen und Männern wieder zu schließen, was mittels der Ergebnisse von PISA und PIAAC (Erwachsene zwischen 16 - 64 Jahren) sowie aus Beitrag 1 geschlussfolgert werden kann (vgl. OECD, 2016; Solheim \& Lundetræ, 2017). Ein direkter Vergleich der Studien ist indes nicht möglich, da verschiedene Instrumente zur Messung der Lesekompetenz verwendet werden und die Skalierung der Lesekompetenz nicht auf einer einheitlichen Skala erfolgt (siehe dazu auch Artelt et al., 2009; OECD, 2013; Solheim \& Lundetræ, 2017). Dennoch deuten gesammelten die Befunde darauf hin, dass der Unterschied in der Lesekompetenz zwischen jungen Frauen und Männern zum Ende der Pflichtschulzeit am höchsten ausfällt. Der Befund für die jungen Erwachsenen zum Ende der neunten Klasse in Beitrag 1 zeigt eine Effektstärke von $d=-0.26$. Ein ebenso vergleichbarer Effekt in der Höhe ist für die deutsche PISA-Stichprobe festzustellen $(d=0.24)$. Gemäß der Interpretation der Effektstärken nach Cohen (1988) entspricht dies einem kleinen Effekt. Auch in der Argumentation der „Gender similarities hypothesis“ nach Hyde $(2005,2014)$ wäre dies ein kleiner und zu vernachlässigender Effekt. Jedoch weist die Autorin in ihrer Ausführung ebenfalls auf die Bedeutung des Kontextes hin, in dem der jeweilige Effekt gefunden wird.

Dieser genannte Kontext und die Interpretation der Ergebnisse soll an dieser Stelle nochmal diskutiert werden. Auch wenn der Effekt in den Unterschieden der Lesekompetenz zwischen jungen Frauen und Männern als nur klein benannt werden kann, hat dies möglicherweise weitreichende Konsequenzen. Über die wiederkehrende Reproduktion von Ergebnissen werden Stereotype darüber, welche Konnotation welcher Gegenstandsbereich haben soll und wer als begabter darin gilt, weiter aufrechterhalten. Daraus resultiert als mögliche Konsequenz, dass Jungen und Männer ein höheres Interesse für MINT-Fächer und Berufe haben und sich Mädchen und Frauen eher in den sozialen sowie sprachlich und künstlerischen Domänen verorten (u. a. Heyder \& Kessels, 2013; Steffens \& Jelenec, 2011). Stanat und Kunter (2002) konnten aufführen, dass sich der Lesekompetenzunterschied in PISA 2000 zwischen 15-jährigen jungen Frauen und Männern nach Kontrolle des Leseinteresses deutlich verringert hat. Dieses Ergebnis verdeutlicht, dass es beim Unterschied in der Lesekompetenz zwischen Jungen/Männern und Mädchen/Frauen weniger um die tatsächliche Kompetenz im Lesen geht, sondern mehr um die Konnotation der Tätigkeit und die Einstellung der Jugendlichen zum Lesen. Möglicherweise tragen ferner die wissenschaftliche Argumentation und Auseinandersetzung dazu bei, dass Stereotype 
hinsichtlich des Lesens weiter erhalten bleiben. Durch die immer wieder gefundenen Ergebnisse der Lesekompetenzunterschiede zwischen Jungen und Mädchen und deren Kommunikation bleibt die Wahrnehmung sowohl in der Forschung als auch in der Öffentlichkeit erhalten, dass Jungen die Verlierer im Lesen sind. Daher sollte bei den Darstellungen der Ergebnisse und im wissenschaftlichen Diskurs die Kommunikationswege und deren Wirkung bedacht werden. Bei der Darstellung von Unterschieden zwischen Mädchen/Frauen und Jungen/Männern sollte zum Beispiel darauf geachtet werden, eine spezifische Gruppe im Vergleich zur anderen nicht immer wiederkehrend als verlierend und benachteiligt darzustellen. Gleichzeitig sollten Ergebnisse wie diejenigen von Hyde (2005, 2014) gefordert, realistisch interpretiert und im jeweiligen Kontext eingebettet betrachtet werden. Mögliche Erklärungen für Unterschiede sollten differenziert dargestellt und vor allem Stärken genauer beschrieben werden, statt einen defizitären Ansatz zu verfolgen.

Insgesamt kann hinsichtlich der beiden Zielsetzungen zur Entwicklung von Geschlechtsunterschieden in der Lesekompetenz und die personen- und textsortenspezifische Determinanten auf die Lesekompetenz von Frauen und Männern folgendes geschlussfolgert werden: Wie in der ersten Forschungsfrage formuliert, konnte auch nach der Schulzeit eine Weiterentwicklung der Lesekompetenz von jungen Frauen und Männern zwischen 16 bis 22 Jahren belegt werden, mit dem durchaus positivem Ergebnis, dass im jungen Erwachsenenalter keine Geschlechtsunterschiede in der Lesekompetenz mehr vorhanden sind. Außerdem konnte in den Beiträgen mit erwachsenen Teilnehmenden bestätigt werden, dass keine Lesekompetenzunterschiede zwischen Frauen und Männern vorhanden sind.

Hinsichtlich der Determinanten der Lesekompetenz von Erwachsenen, kann für die zweite Forschungsfrage geschlussfolgert werden, dass, ebenso wie im Kindes- und Jugendalter, ein positiver Zusammenhang zwischen dem Leseverhalten und der -kompetenz vorhanden ist. Jedoch scheint das informierende Lesen für Erwachsene an Bedeutung zu gewinnen. Anders als ursprünglich angenommen, zeigt sich für die dritte Forschungsfrage, dass ein geschlechtsstereotyper Textinhalt keinen Einfluss auf die Lesekompetenz von Männern und Frauen hat. Obwohl es weiterhin sozial geteilte Annahmen darüber gibt, was typisch weibliche und männliche Eigenschaften sind bzw. wie Frauen und Männer sein sollten, haben diese über den Textinhalt keinen Einfluss auf die Lesekompetenz. 
Artelt und Kolleginnen sowie Kollegen (2007) empfehlen in der Expertise zur Förderung der Lesekompetenz diese als ganzheitlichen Prozess $\mathrm{zu}$ sehen, welcher beginnend mit der Vorschulzeit bis zum Ende der Schulzeit fortgesetzt werden sollte. Allerdings wird anhand der Ergebnisse der vorliegenden Arbeit deutlich, dass die Schulzeit nicht das Ende der Förderung der Lesekompetenz sein sollte. Vielmehr sollte der Prozess der Entwicklung der Lesekompetenz und deren Förderung als lebenslanger aktiver und interaktiver Lernprozess verstanden werden.

Abschließend lässt sich resümierend konstatieren, dass sowohl aufgrund der noch unklaren Befunden zur Lesekompetenzentwicklung über die Lebensspanne und der abweichenden Ergebnisse $\mathrm{zu}$ den Einflussfaktoren auf die Lesekompetenz, weitere Forschung im Übergang zwischen dem Schul- und dem jungen Erwachsenalter sowie dem Erwachsenenalter durchgeführt werden sollten. Unter dem Aspekt des Lesens als Schlüsselkompetenz und der damit verbundenen erfolgreichen gesellschaftlichen Partizipation sollte die Lesekompetenzforschung und deren Förderung als lebenslange Aufgabe angesehen werden. 


\section{Literaturverzeichnis}

Alexander, P. A. (2005). The path to competence: A lifespan developmental perspective on reading. Journal of Literacy Research, 37 (4), 413-436. doi.org/10.1207/s15548430j1r3704_1

Allison, P. D. (2009). Missing data. Thousand Oaks, California: SAGE Publications.

Artelt, C., Drechsel, B., Bos, W. \& Stubbe, T. C. (2009). Lesekompetenz in PISA und PIRLS/IGLU - ein Vergleich. In M. Prenzel \& J. Baumert (Hrsg.), Vertiefende Analysen zu PISA 2006 (Zeitschrift für Erziehungswissenschaft/special issue, S. 35-52). Wiesbaden: VS Verlag für Sozialwissenschaften. doi.org/10.1007/978-3-53 1-9181503

Artelt, C., McElvany, N., Christmann, U., Richter, T., Groeben, N., Köster, J. et al. (2007). Förderung von Lesekompetenz - Expertise (Bildungsforschung, Bd. 17). Berlin: Bundesministerium für Bildung und Forschung.

Artelt, C. \& Schlagmüller, M. (2004). Der Umgang mit literarischen Texten als Teilkompetenz im Lesen? Dimensionsanalysen und Ländervergleiche. In U. Schiefele, C. Artelt, W. Schneider \& P. Stanat (Hrsg.), Struktur, Entwicklung und Förderung von Lesekompetenz. Vertiefende Analysen im Rahmen von PISA 2000 (S. 169-196).

Wiesbaden: VS Verlag für Sozialwissenschaften.

Artelt, C., Stanat, P., Schneider, W. \& Schiefele, U. (2001). Lesekompetenz: Testkonzeption und Ergebnisse. In J. Baumert, E. Klieme, M. Neubrand, M. Prenzel, U. Schiefele, W. Schneider et al. (Hrsg.), PISA 2000. Basiskompetenzen von Schülerinnen und Schülern im internationalen Vergleich (S. 69-137). Wiesbaden: VS Verlag für Sozialwissenschaften. doi.org/10.1007/978-3-322-83412-6_4

Athenstaedt, U. (2000). Normative Geschlechtsrollenorientierung: Entwicklung und Validierung eines Fragebogens. Zeitschrift für Differentielle und Diagnostische Psychologie, 21 (1), 91-104. doi.org/10.1024//0170-1789.21.1.91

Athenstaedt, U. \& Alfermann, D. (2011). Geschlechterrollen und ihre Folgen. Eine sozialpsychologische Betrachtung (Sozial-, Persönlichkeits-, Arbeits- und Organisationspsychologie, 1. Auflage). Stuttgart: Kohlhammer.

Baron, R. A., Markman, G. D. \& Hirsa, A. (2001). Perceptions of women and men as entrepreneurs. Evidence for differential effects of attributional augmenting. Journal of Applied Psychology, 86 (5), 923-929. doi.org/10.1037/0021-9010.86.5.923

Baumert, J., Nagy, G. \& Lehmann, R. (2012). Cumulative advantages and the emergence of social and ethnic inequality. Matthew effects in reading and mathematics development within elementary schools? Child Development, 83 (4), 1347-1367. doi.org/10.1111/j.1467-8624.2012.01779.x 
Becker, M. \& McElvany, N. (2018). The interplay of gender and social background: A longitudinal study of interaction effects in reading attitudes and behaviour. The British journal of educational psychology, 88 (4), 529-549. doi.org/10.1111/bjep. 12199

Becker, M., McElvany, N. \& Kortenbruck, M. (2010). Intrinsic and extrinsic reading motivation as predictors of reading literacy. A longitudinal study. Journal of Educational Psychology, 102 (4), 773-785. doi.org/10.1037/a0020084

Becker, S., Pfost, M. \& Artelt, C. (2018). New challenge, new motivation? Goal orientation development in graduates of higher track schools and their peers in vocational training. Frontiers in Psychology, 9, 1371. doi.org/10.3389/fpsyg. 2018.01371

Becker, S., Pfost, M., Schiefer, I. M. \& Artelt, C. (2017). Ein Motivationsschub durch die Ausbildung? Entwicklung von Zielorientierungen von Beginn der Sekundarstufe I bis nach dem Übergang in die Sekundarstufe II oder in das duale Ausbildungssystem. Zeitschrift für Entwicklungspsychologie und Pädagogische Psychologie, 49 (4), 210 223. doi.org/10.1026/0049-8637/a000182

Becker-Mrotzek, M., Lindauer, T., Pfost, M., Weis, M., Strohmaier, A. \& Reiss, K. (2019). Lesekompetenz heute - eine Schlüsselqualifikation im Wandel. In K. Reiss, M. Weis, E. Klieme \& O. Köller (Hrsg.), PISA 2018. Grundbildung im internationalen Vergleich (1. Auflage, S. 21-46). Münster: Waxmann.

Berendes, K., Becker, M., Jacoby, J., Flunger, B., Nagengast, B. \& Trautwein, U. (2018). Individuelle Entwicklungsverläufe beim Lesen. Macht das Geschlecht den Unterschied? Zeitschrift für Entwicklungspsychologie und Pädagogische Psychologie, 50 (4), 192208. doi.org/10.1026/0049-8637/a000202

Birnbaum, A. (1968). Some latent trait models and their use in inferring an examinee's ability. In F. M. Lord, M. R. Novick \& A. Birnbaum (Hrsg.), Statistical theories of mental test scores (S. 397-479). Reading, MA: Addison-Wesley.

Blossfeld, H.-P., Roßbach, H.-G. \& von Maurice, J. (Hrsg.). (2011). Education as a lifelong process. The German National Educational Panel Study (NEPS) (Zeitschrift für Erziehungswissenschaft/special issue, Bd. 14). Wiesbaden: VS Verlag für Sozialwissenschaften.

Bronner, K. \& Paulus, S. (2017). Intersektionalität: Geschichte, Theorie und Praxis. Eine Einführung für das Studium der Sozialen Arbeit und der Erziehungswissenschaft (utb Soziale Arbeit, Erziehungswissenschaft, Bd. 4873). Opladen: Verlag Barbara Budrich. Brunner, M., Lang, F. R. \& Lüdtke, O. (2014). Erfassung der fluiden kognitiven Leistungsfähigkeit über die Lebensspanne im Rahmen der National Educational Panel Study: Expertise (NEPS Working Paper Nr. 42). Bamberg: Leibniz-Institut für 
Bildungsverläufe, Nationales Bildungspanel. Verfügbar unter https://www.nepsdata.de/Portals/0/Working\%20Papers/WP_XLII.pdf [Zugriff am: 15.12.2020]

Bürger, S., Kröhne, U. \& Goldhammer, F. (2016). The transition to computer-based testing in large-scale assessments: Investigating (partial) measurement invariance between modes. Psychological test and assessment modeling, 58 (4), 597-616.

Cameron, C. E., Grimm, K. J., Steele, J. S., Castro-Schilo, L. \& Grissmer, D. W. (2015). Nonlinear gompertz curve models of achievement gaps in mathematics and reading. Journal of Educational Psychology, 107 (3), 789-804. doi.org/10.1037/edu0000009

Cerella, J., Poon, L. W. \& Williams, D. M. (1980). Age and the complexity hypothesis. In L. W. Poon (Ed.), Aging in the 1980s. Psychological issues (pp. 332-340). Washington, D.C: American Psychological Association. doi.org/10.1037/10050-024

Chall, J. S. (1983). Stages of reading development. New York, N.Y.: McGraw-Hill Book Co.

Chapman, M., Filipenko, M., McTavish, M. \& Shapiro, J. (2007). First graders' preferences for narrative and/or information books and perceptions of other boys' and girls' book preferences. Canadian Journal of Education/Revue canadienne de l'éducation, 30 (2), 531-553. doi.org/10.2307/20466649

Charlton, M., Burbaum, C. \& Sutter, T. (2004). Lesen Frauen wirklich anders? Oder lesen sie nur eine andere Literatur als Männer? SPIEL, 23 (1), 3-22.

Chiu, M. M. \& McBride-Chang, C. (2006). Gender, context, and reading: A comparison of students in 43 countries. Scientific Studies of Reading, 10 (4), 331-362. doi.org/10.1207/s1532799xssr1004_1

Christin, A. (2012). Gender and highbrow cultural participation in the United States. Poetics, 40 (5), 423-443. doi.org/10.1016/j.poetic.2012.07.003

Christmann, U. \& Groeben, N. (1999). Psychologie des Lesens. In B. Franzmann, K. Hasemann, D. Löffler \& E. Schön (Hrsg.), Handbuch Lesen (S. 145-223). München: Saur.

Christmann, U. \& Groeben, N. (2009). Anforderungen und Einflussfaktoren bei Sach- und Informationstexten. In N. Groeben \& B. Hurrelmann (Hrsg.), Lesekompetenz. Bedingungen, Dimensionen, Funktionen (Lesesozialisation und Medien, 3. Auflage, S. 150-173). Weinheim: Juventa Verlag.

Cohen, J. (1988). Statistical power analysis for the behavioral sciences (2. ed.). Hillsdale, NJ: Erlbaum.

De Fraine, B., Van Damme, J. \& Onghena, P. (2007). A longitudinal analysis of gender differences in academic self-concept and language achievement: A multivariate multilevel latent growth approach. Contemporary Educational Psychology, 32 (1), 132150. doi.org/10.1016/j.cedpsych.2006.10.005 
Diedrich, J., Schiepe-Tiska, A., Ziernwald, L., Tupac-Yupanqui, A., Weis, M., McElvany, N. et al. (2019). Lesebezogene Schülermerkmale in PISA 2018: Motivation, Leseverhalten, Selbstkonzept und Lesestrategiewissen. In K. Reiss, M. Weis, E. Klieme \& O. Köller (Hrsg.), PISA 2018. Grundbildung im internationalen Vergleich (1. Auflage, S. 80-110). Münster: Waxmann.

Duncan, L. G., McGeown, S. P., Griffiths, Y. M., Stothard, S. E. \& Dobai, A. (2016). Adolescent reading skill and engagement with digital and traditional literacies as predictors of reading comprehension. British Journal of Psychology, 107 (2), 209-238. doi.org/10.1111/bjop.12134

Eagly, A. H., Nater, C., Miller, D. I., Kaufmann, M. \& Sczesny, S. (2019). Gender stereotypes have changed. A cross-temporal meta-analysis of U.S. public opinion polls from 1946 to 2018. The American psychologist, 75(3), 301-315. doi.org/10.1037/amp0000494

Eccles, J. S. (1987). Gender roles and women's achievement-related decisions. Psychology of Women Quarterly, 11 (2), 135-172. doi.org/10.1111/j.1471-6402.1987.tb00781.x

El-Khechen, W., McElvany, N., Wolter, I. \& Kessels, U. (2017). Was heißt „kannibalisch“, „foltern“ und „zerstören"? Geschlechtsspezifischer Wortschatz von Grundschulkindern in Abhängigkeit von der Familiensprache. Psychologie in Erziehung und Unterricht, 64 (2), 123-137. doi.org/10.2378/peu2017.art06d

Europäische Kommission (Hrsg.). Vorschlags für eine Empfehlung des Rates zu Schlüsselkompetenzen für lebenslanges Lernen. SWD(2018) 14.

Fischer, L., Rohm, T., Gnambs, T. \& Carstensen, C. H. (2016). Linking the data of the competence tests (NEPS Survey Paper Nr. 1). Bamberg: Leibniz Institute for Educational Trajectories, National Educational Panel Study. Verfügbar unter https://www.neps-data.de/Portals/0/Survey\%20Papers/SP_I.pdf [Zugriff am: 15.12.2020]

Garnham, A., Oakhill, J., von Stockhausen, L. \& Sczesny, S. (2016). Editorial: Language, cognition, and gender. Frontiers in Psychology, 7, 772. doi.org/10.3389/fpsyg. 2016.00772

Gehrer, K. \& Artelt, C. (2013). Literalität und Bildungslaufbahn: Das Bildungspanel NEPS. In C. Rosebrock \& A. Bertschi-Kaufmann (Hrsg.), Literalität erfassen . Bildungspolitisch, kulturell, individuell (S. 168-187). Weinheim: Beltz Juventa.

Gehrer, K., Zimmermann, S., Artelt, C. \& Weinert, S. (2013). NEPS framework for assessing reading competence and results from an adult pilot study. Journalfor Educational Research Online, 5 (2), 50-79.

Gnambs, T., Fischer, L. \& Rohm, T. (2017). NEPS technical reportfor reading: scaling results of starting cohort 4 for grade 12 (NEPS Survey Paper Nr. 13). Bamberg: 
Leibniz Institute for Educational Trajectories, National Educational Panel Study. Verfügbar unter https://www.neps-data.de/Portals/0/Survey\%20Papers/SP_XIII.pdf [Zugriff am: 15.12.2020]

Goldhammer, F., Harrison, S., Bürger, S., Kröhne, U., Lüdtke, O., Robitzsch, A. et al. (2019). Vertiefende Analysen zur Umstellung des Modus von Papier auf Computer. In K. Reiss, M. Weis, E. Klieme \& O. Köller (Hrsg.), PISA 2018. Grundbildung im internationalen Vergleich (1. Auflage, S. 163-186). Münster: Waxmann.

Graesser, A. C., Singer, M. \& Trabasso, T. (1994). Constructing inferences during narrative text comprehension. Psychological Review, 101 (3), 371-395. doi.org/10.1037/0033-295X. 101.3.371

Groeben, N. (2004). Einleitung: Funktionen des Lesens - Normen der Gesellschaft. In N. Groeben \& B. Hurrelmann (Hrsg.), Lesesozialisation in der Mediengesellschaft. Ein Forschungsüberblick (Lesesozialisation und Medien, S. 11-35). Weinheim: Beltz Juventa.

Grotlüschen, A., Mallows, D., Reder, S. \& Sabatini, J. (2016). Adults with low proficiency in literacy or numeracy (OECD Education Working Papers No. 131). doi.org/10.1787/5jm0v44bnmnx-en

Guthrie, J. T. \& Wigfield, A. (2009). How Motivation Fits Into a Science of Reading. Scientific Studies of Reading, 3 (3), 199-205. doi.org/10.1207/s1532799xssr0303_1

Guthrie, J. T., Wigfield, A., Metsala, J. L. \& Cox, K. E. (1999). Motivational and Cognitive Predictors of Text Comprehension and Reading Amount. Scientific Studies of Reading, 3 (3), 231-256. doi.org/10.1207/s1532799xssr0303_3

Haberkorn, K., Pohl, S., Hardt, K. \& Wiegand, E. (2012). NEPS technical report for reading - scaling results of starting cohort 4 in ninth grade (NEPS Working Paper Nr. 16). Bamberg: University of Bamberg, National Educational Panel Study. Verfügbar unter https://www.neps-data.de/Portals/0/Working\%20Papers/WP_XVI.pdf [Zugriff am: 15.12.2020]

Haines, E. L., Deaux, K. \& Lofaro, N. (2016). The times they are a-changing ... or are they not? A comparison of gender stereotypes, 1983-2014. Psychology of Women Quarterly, 40 (3), 353-363. doi.org/10.1177/0361684316634081

Haladyna, T. M. \& Rodriguez, M. (2013). Developing and validating test items. New York, NY: Routledge.

Hannover, B. (2006). Geschlechterrollen. In H.-W. Bierhoff \& D. Frey (Hrsg.), Handbuch der Sozialpsychologie und Kommunikationspsychologie (Handbuch der Psychologie, S. 465-470). Göttingen: Hogrefe.

Hannover, B. \& Kessels, U. (2011). Sind Jungen die neuen Bildungsverlierer? Empirische Evidenz für Geschlechterdisparitäten zuungunsten von Jungen und Erklärungsansätze. 
Zeitschriftfür Pädagogische Psychologie, 25 (2), 89-103. doi.org/10.1024/10100652/a000039

Heine, J.-H. \& Reiss, K. (2019). PISA 2018 - die Methodologie. In K. Reiss, M. Weis, E. Klieme \& O. Köller (Hrsg.), PISA 2018. Grundbildung im internationalen Vergleich (1. Auflage, S. 241-258). Münster: Waxmann.

Heyder, A. \& Kessels, U. (2013). Is School Feminine? Implicit Gender Stereotyping of School as a Predictor of Academic Achievement. Sex Roles, 69 (11), 605-617. doi.org/10.1007/s11199-013-0309-9

Hurrelmann, B. (2004). Sozialisation der Lesekompetenz. In U. Schiefele, C. Artelt, W. Schneider \& P. Stanat (Hrsg.), Struktur, Entwicklung und Förderung von Lesekompetenz. Vertiefende Analysen im Rahmen von PISA 2000 (S. 36-60). Wiesbaden: VS Verlag für Sozialwissenschaften.

Hyde, J. S. (2005). The gender similarities hypothesis. American Psychologist, 60 (6), 581-592. doi.org/10.1037/0003-066X.60.6.581

Hyde, J. S. (2014). Gender similarities and differences. Annual Review of Psychology, 65, 373-398. doi.org/10.1146/annurev-psych-010213-115057

Kintsch, W. (1998). Comprehension. A paradigm for cognition . Cambridge: Cambridge Univ. Press.

Klicpera, C. \& Gasteiger-Klicpera, B. (1993). Lesen und Schreiben. Entwicklung und Schwierigkeiten; die Wiener Längsschnittuntersuchungen über die Entwicklung, den Verlauf und die Ursachen von Lese-und Schreibschwierigkeiten in der Pflichtschulzeit (1. Auflage). Bern: Huber.

Klieme, E. \& Hartig, J. (2008). Kompetenzkonzepte in den Sozialwissenschaften und im erziehungswissenschaftlichen Diskurs. In M. Prenzel, I. Gogolin \& H.-H. Krüger (Hrsg.), Kompetenzdiagnostik (S. 11-29). Wiesbaden: VS Verlag für Sozialwissenschaften. doi.org/10.1007/978-3-531-90865-6_2

Kröhne, U., Bürger, S., Hahnel, C. \& Goldhammer, F. (2019). Construct equivalence of PISA reading comprehension measured with paper-based and computer-based assessments. Educational Measurement: Issues and Practice, 31 (1), 1-15. doi.org/10.1111/emip. 12280

Kröhne, U., Hahnel, C. \& Goldhammer, F. (2019). Invariance of the response processes between gender and modes in an assessment of reading. Frontiers in Applied Mathematics and Statistics, 5, 2. doi.org/10.3389/fams. 2019.00002

Kröhne, U. \& Martens, T. (2011). Computer-based competence tests in the National Educational Panel Study: The challenge of mode effects. In H.-P. Blossfeld, H.-G. Roßbach \& J. von Maurice (Hrsg.), Education as a lifelong process. The German National Educational Panel Study (NEPS) (Zeitschrift für 
Erziehungswissenschaft/special issue, Bd. 14, S. 169-186). Wiesbaden: VS Verlag für Sozialwissenschaften.

Lafontaine, D. \& Monseur, C. (2009). Gender gap in comparative studies of reading comprehension: to what extent do the test characteristics make a difference. European Educational Research Journal, 8 (1), 69-79. doi.org/10.2304/eerj.2009.8.1.69

Lagaert, S., van Houtte, M. \& Roose, H. (2017). Engendering culture: The relationship of gender identity and pressure for gender conformity with adolescents' interests in the arts and literature. Sex Roles, 77 (7-8), 482-495. doi.org/10.1007/s11199-017-0738-y

Lenhard, W. (2019). Leseverständnis und Lesekompetenz. Grundlagen - Diagnostik Förderung (2., aktualisierte Auflage). Stuttgart: Kohlhammer.

Lenhard, W. \& Artelt, C. (2009). Komponenten des Leseverständnisses. In W. Lenhard (Hrsg.), Diagnostik und Förderung des Leseverständnisses (Tests und Trends, Bd. 7, S. 1-17). Göttingen: Hogrefe.

Locher, F. M. \& Pfost, M. (2018). Erfassung des Lesevolumens in Large-Scale Studien. Diagnostica, 1-11. doi.org/10.1026/0012-1924/a 000203

Locher, F. M. \& Pfost, M. (2019). The relation between time spent reading and reading comprehension throughout the life course. Journal of Research in Reading, 43 (1), 57 77. doi.org/10.1111/1467-9817. 12289

Logan, S. \& Johnston, R. (2009). Gender differences in reading ability and attitudes: examining where these differences lie. Journal of Research in Reading, 32 (2), 199 214. doi.org/10.1111/j.1467-9817.2008.01389.x

Lundetræ, K., Sulkunen, S., Gabrielsen, E. \& Malin, A. (2014). A comparison of PIAAC and PISA results. In A. Malin (Hrsg.), Associations between age and cognitive foundation skills in the Nordic countries: A closer look at the data (S. 171-187). Jyväskylä: University of Jyväskylä, Finnish Institute for Educational Research. Verfügbar unter https://jyx.jyu.fi/handle/123456789/44924 [Zugriff am: 15.12.2020] MacKinnon, D. P., Cheong, J. \& Pirlott, A. G. (2012). Statistical mediation analysis. In H. Cooper, P. M. Camic, D. L. Long, A. T. Panter, D. Rindskopf \& K. J. Sher (Hrsg.), APA Handbook of Research Methods in Psychology, Vol. 2: Research Designs: Quantita tive, Qualitative, Neuropsychological, and Biological (S. 313-331). Washington: American Psychological Association. doi.org/10.1037/13620-018

Maehler, D. B., Massing, N., Helmschrott, S., Rammstedt, B., Staudinger, U. M. \& Wolf, C. (2013). Grundlegende Kompetenzen in verschiedenen Bevölkerungsgruppen. In B. Rammstedt (Hrsg.), Grundlegende Kompetenzen Erwachsener im internationalen Vergleich. Ergebnisse von PIAAC 2012 (S. 77-126). Münster: Waxmann. 
Mar, R. A. \& Rain, M. (2015). Narrative fiction and expository nonfiction differentially predict verbal ability. Scientific Studies of Reading, 19 (6), 419-433. doi.org/10.1080/10888438.2015.1069296

Martiny, S. E. \& Götz, T. (2011). Stereotype Threat in Lern- und Leistungssituationen : theoretische Ansätze, empirische Befunde und praktische Implikationen. In M. Dresel\& L. Lämmle (Hrsg.), Motivation, Selbstregulation und Leistungsexzellenz (Talentförderung, Expertiseentwicklung, Leistungsexzellenz, Bd. 9, S. 153-178). Münster: LIT.

McElvany, N. \& Becker, M. (2010). Welche Prädiktoren braucht man zur Vorhersage von Lesekompetenz? Eine Kommunalitätenanalyse zur Bestimmung der uniquen und geteilten Varianzaufklärung psychologischer und soziologischer Konstrukte. In W. Bos, E. Klieme \& O. Köller (Hrsg.), Schulische Lerngelegenheiten und Kompetenzentwicklung: Festschrift für Jürgen Baumert (S. 147-172). Münster: Waxmann.

McElvany, N., El-Khechen, W., Schwabe, F. \& Kessels, U. (2016). Qualitative Wortschatzunterschiede zwischen Mädchen und Jungen im Grundschulalter. Zeitschrift für Pädagogische Psychologie, 30 (1), 45-55. doi.org/10.1024/101 0-0652/a000167

McElvany, N., Kessels, U., Schwabe, F. \& Kasper, D. (2017). Geschlecht und Lesekompetenz. In A. Hußmann, H. Wendt, W. Bos, A. Bremerich-Vos, D. Kasper, E.M. Lankes et al. (Hrsg.), IGLU 2016. Lesekompetenzen von Grundschulkindern in Deutschland im internationalen Vergleich (S. 177-194). Münster: Waxmann.

McElvany, N., Kortenbruck, M. \& Becker, M. (2008). Lesekompetenz und Lesemotivation. Entwicklung und Mediation des Zusammenhangs durch Leseverhalten. Zeitschriftfür Pädagogische Psychologie, 22 (3-4), 207-219. doi.org/10.1024/10100652.22.34.207

McElvany, N. \& Schwabe, F. (2019). Gender gap in reading digitally? Examining the role of motivation and self-concept. Journal for Educational Research Online, 11 (1), 145165.

McGeown, S. P., Goodwin, H., Henderson, N. \& Wright, P. (2012). Gender differences in reading motivation: Does sex or gender identity provide a better account? Journal of Research in Reading, 35 (3), 328-336. doi.org/10.1111/j.1467-9817.2010.01481.x

McGeown, S. P. (2015). Sex or gender identity? Understanding children's reading choices and motivation. Journal of Research in Reading, 38 (1), 35-46. doi.org/10.1111/j.14679817.2012.01546.x

McGeown, S. P., Duncan, L. G., Griffiths, Y. M. \& Stothard, S. E. (2015). Exploring the relationship between adolescent's reading skills, reading motivation and reading habits. Reading and Writing, 28 (4), 545-569. doi.org/10.1007/s11145-014-9537-9 
Miyamoto, A., Pfost, M., \& Artelt, C. (2018). Reciprocal relations between intrinsic reading motivation and reading competence: a comparison between native and immigrant students in Germany. Journal of Research in Reading, 41(1), 176-196. doi.org/10.1111/1467-9817.12113

Mol, S. E. \& Bus, A. G. (2011). To read or not to read: A meta-analysis of print exposure from infancy to early adulthood. Psychological Bulletin, 137 (2), 267-296. doi.org/10.1037/a0021890

Mol, S. E., Bus, A. G., de Jong, M. T. \& Smeets, D. J. H. (2008). Added Value of Dialogic Parent-Child Book Readings. A Meta-Analysis. Early Education and Development, 19 (1), 7-26. doi.org/10.1080/10409280701838603

Möller, J. \& Schiefele, U. (2004). Motivationale Grundlagen der Lesekompetenz. In U. Schiefele, C. Artelt, W. Schneider \& P. Stanat (Hrsg.), Struktur, Entwicklung und Förderung von Lesekompetenz. Vertiefende Analysen im Rahmen von PISA 2000 (S. 101-124). Wiesbaden: VS Verlag für Sozialwissenschaften.

Müller, B. \& Richter, T. (2014). Lesekompetenz. In J. Grabowski (Hrsg.), Sinn und Unsinn von Kompetenzen. Fähigkeitskonzepte im Bereich von Sprache, Medien und Kultur (S. 29-49). Opladen: Verlag Barbara Budrich.

Mullis, I. V. S., Martin, M. O., Foy, P. \& Hopper, M. (2017). PIRLS 2016 international results in reading. Boston. Verfügbar unter http://timssandpirls.bc.edu/pirls2016/international-results/ [Zugriff am: 15.12.2020] Muthén, L. K. \& Muthén, B. O. (2017). Mplus user's guide (Eighth Edition). Los Angeles, CA: Muthén \& Muthén.

Nagy, G., Retelsdorf, J., Goldhammer, F., Schiepe-Tiska, A. \& Lüdtke, O. (2017). Veränderungen der Lesekompetenz von der 9. zur 10. Klasse: Differenzielle Entwicklungen in Abhängigkeit der Schulform, des Geschlechts und des soziodemografischen Hintergrunds? Zeitschrift für Erziehungswissenschaft, 20 (2), 177-203. doi.org/10.1007/s1 1618-017-0747-1

Naumann, J., Artelt, C., Schneider, W. \& Stanat, P. (2010). Lesekompetenz von PISA 2000 bis PISA 2009. In E. Klieme, C. Artelt, J. Hartig, N. Jude, O. Köller, M. Prenzel et al. (Hrsg.), PISA 2009. Bilanz nach einem Jahrzehnt (S. 23-71). Münster: Waxmann.

Naumann, J. \& Sälzer, C. (2017). Digital reading proficiency in german 15-year olds: Evidence from PISA 2012. Zeitschrift für Erziehungswissenschaft, 20 (4), 585-603. doi.org/10.1007/s11618-017-0758-y

Niklas, F. (2015). Die familiäre Lernumwelt und ihre Bedeutung für die kindliche Kompetenzentwicklung. Psychologie in Erziehung und Unterricht, 62 (2), 106. doi.org/10.2378/peu2015.art11d 
Nosek, B. A., Banaji, M. R. \& Greenwald, A. G. (2002). Math = male, me = female, therefore math not $=$ me. Journal of Personality and Social Psychology, 83 (1), 44-59. doi.org/10.1037//0022-3514.83.1.44

Nowicki, E. A. \& Lopata, J. (2017). Children's implicit and explicit gender stereotypes about mathematics and reading ability. Social Psychology of Education, 20 (2), 329345. doi.org/10.1007/s11218-015-9313-y

OECD (1999). Measuring student knowledge and skills: A new framework for assessment. Paris: OECD Publishing. doi.org/10.1787/9789264173125-en

OECD (2013). The survey of adult skills: Reader's companion. Paris: OECD Publishing. doi.org/10.1787/9789264204027-en

OECD (2016). Skills matter: Further results from the survey of adult skills. Paris: OECD Publishing. doi.org/10.1787/9789264258051-en

OECD (2019). PISA 2018 Ergebnisse (Band I): OECD Publishing. doi.org/10.1787/1 da50379-de

Pfost, M. \& Artelt, C. (2013). Reading literacy development in secondary school and the effect of differential institutional learning environments. In M. Pfost, C. Artelt \& S. Weinert (Eds.), The development of reading literacy from early childhood to adolescence. Empirical findings from the Bamberg BiKS longitudinal studies (Schriften aus der Fakultät Humanwissenschaften der Otto-Friedrich-Universität Bamberg, vol. 14, 229-277). Bamberg: University of Bamberg Press.

Pfost, M., Dörfler, T. \& Artelt, C. (2010). Der Zusammenhang zwischen außerschulischem Lesen und Lesekompetenz. Ergebnisse einer Längsschnittstudie am Übergang von der Grund- in die weiterführende Schule. Zeitschrift für Entwicklungspsychologie und Pädagogische Psychologie, 42 (3), 167-176. doi.org/10.1026/0049-8637/a000017

Pfost, M., Dörfler, T. \& Artelt, C. (2013). Students' extracurricular reading behavior and the development of vocabulary and reading comprehension. Learning and Individual Differences, 26, 89-102. doi.org/10.1016/j. lindif.2013.04.008

Pfost, M., Hattie, J., Dörfler, T. \& Artelt, C. (2014). Individual differences in reading development: A review of 25 years of empirical research on Matthew effects in reading. Review of Educational Research, 84 (2), 203-244. doi.org/10.3102/0034654313509492

Philipp, M. (2011). Lesesozialisation in Kindheit und Jugend. Lesemotivation, Leseverhalten und Lesekompetenz in Familie, Schule und Peer-Beziehungen. Stuttgart: Kohlhammer.

Philipp, M. (2015). Geschlecht und Lesen. In U. Rautenberg \& U. Schneider (Hrsg.), Lesen. Ein interdisziplinäres Handbuch (445-467). Berlin: De Gruyter.

Philipp, M. (2018). Lesekompetenz bei multiplen Texten. Grundlagen, Prozesse, Didaktik (UTB). Tübingen: A. Francke Verlag. 
Pohl, S. \& Carstensen, C. H. (2013). Scaling of competence tests in the National Educational Panel Study - Many questions, some answers, and further challenges. Journal for Educational Research Online, 5 (2), 189-216.

Retelsdorf, J., Becker, M., Köller, O. \& Möller, J. (2012). Reading development in a tracked school system: A longitudinal study over 3 years using propensity score matching. The British journal of educational psychology, 82 (Pt 4), 647-671. doi.org/10.1111/j.2044-8279.2011.02051.x

Retelsdorf, J., Köller, O. \& Möller, J. (2011). On the effects of motivation on reading performance growth in secondary school. Learning and Instruction, 21 (4), 550-559. doi.org/10.1016/j. learninstruc.2010.11.001

Rexing, V., Keimes, C. \& Ziegler, B. (2015). Promotion of reading skills with recipient and domain specific focus - empirical findings in the professional field of construction engineering. Journal of Technical Education, 3 (1), 56-74.

Richter, T. \& Christmann, U. (2009). Lesekompetenz: Prozessebene und interindividuelle Unterschiede. In N. Groeben \& B. Hurrelmann (Hrsg.), Lesekompetenz. Bedingungen, Dimensionen, Funktionen (Lesesozialisation und Medien, 3. Auflage, S. 25-58). Weinheim: Juventa Verlag.

Robinson, J. P. \& Lubienski, S. T. (2011). The development of gender achievement gaps in mathematics and reading during elementary and middle school. American Educational Research Journal, 48 (2), 268-302. doi.org/10.3102/0002831210372249

Robitzsch, A., Lüdtke, O., Köller, O., Kröhne, U., Goldhammer, F. \& Heine, J.-H. (2017). Herausforderungen bei der Schätzung von Trends in Schulleistungsstudien. Eine Skalierung der deutschen PISA-Daten. Diagnostica, 63 (2), 148-165. doi.org/10.1026/0012-1924/a000177

Rohm, T., Scharl, A., Ettner, J. \& Gehrer, K. (2019). NEPS Technical Report for Reading: Scaling Results of Starting Cohorts 4 (Wave 10), 5 (Wave 12), and 6 (Wave 9). (NEPS Survey Paper Nr. 62). Bamberg: Leibniz Institute for Educational Trajectories, National Educational Panel Study. Verfügbar unter https://www.nepsdata.de/Portals/0/Survey\%20Papers/SP_LXII.pdf [Zugriff am: 15.12.2020]

Rosebrock, C. (2016). Anforderungen von Sach- und Informationstexten, Anforderungen literarischer Texte. In A. Bertschi-Kaufmann \& T. Graber (Hrsg.), Lesekompetenz Leseleistung - Leseförderung. Grundlagen, Modelle und Materialien (Lehren lernen, 6. Auflage, S. 58-75). Zug: Klett und Balmer Verlag; Klett-Kallmeyer.

Rost, D. H. \& Buch, S. R. (2018). Leseverständnis. In D. H. Rost, J. R. Sparfeldt \& S. Buch (Hrsg.), Handwörterbuch pädagogische Psychologie (5., überarbeitete und erweiterte Auflage, S. 507-520). Weinheim: Beltz. 
Ryan, R. M. \& Deci, E. L. (2000). Intrinsic and extrinsic motivations: Classic definitions and new directions. Contemporary Educational Psychology, 25 (1), 54-67. doi.org/10.1006/ceps.1999.1020

Sälzer, C. \& Reiss, K. (2016). PISA 2015 - die aktuelle Studie. In K. Reiss, C. Sälzer, A. Schiepe-Tiska, E. Klieme \& O. Köller (Hrsg.), PISA 2015. Eine Studie zwischen Kontinuität und Innovation (S. 13-43). Münster: Waxmann.

Schaffner, E., Philipp, M. \& Schiefele, U. (2016). Reciprocal effects between intrinsic reading motivation and reading competence? A cross-lagged panel model for academic track and nonacademic track students. Journal of Research in Reading, 39 (1), 19-36. doi.org/10.1111/1467-9817.12027

Schiefele, U. (1996). Motivation und Lernen mit Texten. Göttingen: Hogrefe.

Schiefele, U., Schaffner, E., Möller, J. \& Wigfield, A. (2012). Dimensions of reading motivation and their relation to reading behavior and competence. Reading Research Quarterly, 47 (4), 427-463. doi.org/10.1002/RRQ.030

Schipolowski, S., Wittig, J., Weirich, S. \& Böhme, K. (2017). Geschlechtsbezogene Disparitäten. In P. Stanat, S. Schipolowski, C. Rjosk, S. Weirich \& N. Haag (Hrsg.), IQB-Bildungstrend 2016. Kompetenzen in den Fächern Deutsch und Mathematik am Ende der 4. Jahrgangsstufe im zweiten Ländervergleich (S. 187-212). Münster: Waxmann.

Schmidt-Hertha, B. (2018). Kompetenzerwerb im Lebenslauf - Ergebnisse aus PIAAC und CiLL. In C. Hof, H. Rosenberg \& W. Seitter (Hrsg.), Lernen im Lebenslauf. Theoretische Perspektiven und empirische Zugänge (Theorie und Empirie Lebenslangen Lernens, S. 121-137). Wiesbaden: Springer VS.

Schnotz, W. (2006). Was geschieht im Kopf des Lesers? Mentale Konstruktionsprozesse beim Textverstehen aus der Sicht der Psychologie und der kognitiven Linguistik. In $\mathrm{H}$. Blühdorn, E. Breindl \& U. H. Waßner (Hrsg.), Text - Verstehen. Grammatik und darüber hinaus (Jahrbuch Institut für Deutsche Sprache, S. 222-238). Berlin: De Gruyter. doi.org/10.1515/9783110199963.2.222

Schreier, M. \& Odağ, Ö. (2004). Genuss und Distanz: Geschlechtsspezifische Kompetenzen beim Umgang mit Realität(en) und Fiktion(en). SPIEL, 23 (1), 94-115. Schwabe, F., McElvany, N. \& Trendtel, M. (2015). The school age gender gap in reading achievement: Examining the influences of item format and intrinsic reading motivation. Reading Research Quarterly, 50 (2), 219-232. doi.org/10.1002/rrq.92

Senko, C. \& Dawson, B. (2017). Performance-approach goal effects depend on how they are defined. Meta-analytic evidence from multiple educational outcomes. Journal of Educational Psychology, 109 (4), 574-598. doi.org/10.1037/edu0000160 
Solheim, O. J. \& Lundetræ, K. (2017). Can test construction account for varying gender differences in international reading achievement tests of children, adolescents and young adults? - A study based on Nordic results in PIRLS, PISA and PIAAC. Assessment in Education: Principles, Policy \& Practice, 3, 1-20. doi.org/10.1080/0969594X.2016.1239612

Stanat, P. \& Kunter, M. (2002). Geschlechterspezifische Leistungsunterschiede bei Fünfzehnjährigen im internationalen Vergleich. Zeitschriftfür Erziehungswissenschaft, 4 (1), 28-48. doi.org/10.1007/s 11618-002-0003-0

Steffens, M. C. \& Jelenec, P. (2011). Separating implic it gender stereotypes regarding math and language: Implicit ability stereotypes are self-serving for boys and men, but not for girls and women. Sex Roles, 64 (5-6), 324-335. doi.org/10.1007/s 11199-0109924-X

Steyer, R., Eid, M. \& Schwenkmezger, P. (1997). Modeling true intraindividual change: True change as a latent variable. Methods of Psychological Research, 2 (1), 21-33.

Steyer, R., Partchev, I. \& Shanahan, M. J. (2000). Modeling true intraindividual change in structural equation models: The case of poverty and children's psychosocial adjustment. In T. D. Little, K. U. Schnabel \& J. Baumert (Hrsg.), Modeling longitudinal and multilevel data: Practical issues, applied approaches, and specific examples (S. 109126). Hillsdale, New York: Erlbaum.

Stiftung Lesen (2008). Lesen in Deutschland 2008, Mainz.

Tippelt, R. (2018). Lebenslanges Lernen als Kompetenzentwicklung. In C. Hof, H.

Rosenberg \& W. Seitter (Hrsg.), Lernen im Lebenslauf. Theoretische Perspektiven und empirische Zugänge (Theorie und Empirie Lebenslangen Lernens, S. 105-120).

Wiesbaden: Springer VS.

Vágvölgyi, R., Coldea, A., Dresler, T., Schrader, J. \& Nuerk, H.C. (2016). A review about functional illiteracy: Definition, cognitive, linguistic, and numerical aspects. Frontiers in Psychology, 7, Art. 1617. doi.org/10.3389/fpsyg.2016.01617

Van Dijk, T. A. \& Kintsch, W. (1983). Strategies of discourse comprehension. New York: Academic Press.

Watson, A., Kehler, M. \& Martino, W. (2010). The problem of boys' literacy underachievement. Raising some questions. Journal of Adolescent \& Adult Literacy, 53 (5), 356-361. doi.org/10.1598/JAAL.53.5.1

Weinert, F. E. (2001). Concept of competence: A conceptual clarification. In D. S. Rychen \& L. H. Salganik (Hrsg.), Defining and selecting key competencies (S. 45-66). Seattle: Hogrefe and Huber.

Weinert, S., Artelt, C., Prenzel, M., Senkbeil, M., Ehmke, T. \& Carstensen, C. H. (2011). Development of competencies across the life span. In H.-P. Blossfeld, H.-G. Roßbach \& 
J. von Maurice (Hrsg.), Education as a lifelong process. The German National

Educational Panel Study (NEPS) (Zeitschrift für Erziehungswissenschaft/special issue,

Bd. 14, S. 67-86). Wiesbaden: VS Verlag für Sozialwissenschaften.

doi.org/10.1007/s11618-011-0182-7

Weinert, S. \& Ebert, S. (2013). Spracherwerb im Vorschulalter. Zeitschrift für

Erziehungswissenschaft, 16 (2), 303-332. doi.org/10.1007/s11618-013-0354-8

Weis, M., Doroganova, A., Hahnel, C., Becker-Mrotzek, M., Lindauer, T., Artelt, C. et al. (2019). Lesekompetenz in PISA 2018 - Ergebnisse in einer digitalen Welt. In K. Reiss, M. Weis, E. Klieme \& O. Köller (Hrsg.), PISA 2018. Grundbildung im internationalen Vergleich (1. Auflage, S. 47-80). Münster: Waxmann.

Weis, M., Müller, K., Mang, J., Heine, J.-H., Mahler, N. \& Reiss, K. (2019). Soziale Herkunft, Zuwanderungshintergrund und Lesekompetenz. In K. Reiss, M. Weis, E. Klieme \& O. Köller (Hrsg.), PISA 2018. Grundbildung im internationalen Vergleich (1. Auflage, S. 129-162). Münster: Waxmann.

Wicht, A., Rammstedt, B. \& Lechner, C. M. (2020). Predictors of Literacy Development in Adulthood. Insights from a Large-scale, Two-wave Study. Scientific Studies of Reading, 42, 1-9. doi.org/10.1080/10888438.2020.1751635

Wigfield, A., Eccles, J. S., Roeser, R. W. \& Schiefele, U. (2008). Development of achievement motivation. In W. Damon \& R. M. Lerner (Eds.), Child and adolescent development. An advanced course (pp. 406-434). Hoboken, NJ: Wiley.

Wigfield, A. \& Guthrie, J. T. (1997). Motivation for reading: An overview. Educational Psychologist, 32 (2), 57-58.

Wirthwein, L., Sparfeldt, J. R., Pinquart, M., Wegerer, J. \& Steinmayr, R. (2013). Achievement goals and academic achievement. A closer look at moderating factors. Educational Research Review, 10, 66-89. doi.org/10.1016/j.edurev.2013.07.001

Zinn, S. \& Gnambs, T. (2018). Modeling competence development in the presence of selection bias. Behavior research methods, 50 (6), 1-16. doi.org/10.3758/s 13428-018$1021-\mathrm{z}$ 


\section{Anhang}

\section{Verzeichnis der Beiträge}

\section{Beitrag 1}

Thums, K.; Schwabe, F.; Sengewald, M.-A.; Artelt, C.; Wolter, I. (eingereicht). When do gender differences in reading competence diminish? A longitudinal study of women and men from adolescence to young adulthood.

\section{Beitrag 2}

Thums, K., Artelt, C., \& Wolter, I. (2020). Reading for entertainment or information reception? Gender differences in reading preferences and their impact on text-type-specific reading competences in adult readers. European Journal of Psychology of Education. Advance online publication. doi:10.1007/s10212-020-00486-1

\section{Beitrag 3}

Thums, K., Gnambs, T., \& Wolter, I. (2020). The impact of gender-stereotypical text contents on reading competence in women and men. Zeitschrift für Erziehungswissenschaft, 23(6), 1283-1301. doi.org/10.1007/s11618-020-00980-8 


\section{Beitrag 1}

Thums, K.; Schwabe, F.; Sengewald, M.-A.; Artelt, C.; Wolter, I. (eingereicht). When do gender differences in reading competence diminish? A longitudinal study of women and men from adolescence to young adulthood. 
When do gender differences in reading competence diminish? A longitudinal study of women and men from adolescence to young adulthood.

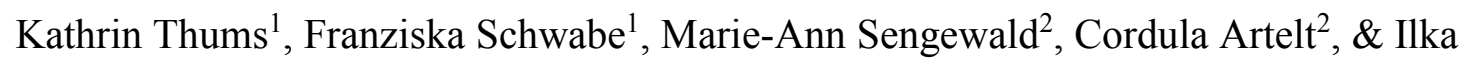
Wolter $^{2}$

1 Centre for Research on Education and School Development (IFS), Dortmund, Germany

2 Leibniz Institute for Educational Trajectories, Bamberg, Germany

Corresponding author:

Kathrin Thums, Centre for Research on Education and School Development (IFS),

Vogelpothsweg 78, 44227 Dortmund, Germany, kathrin.thums@tu-dortmund.de, ORCID

iD 0000-0002-4285-0236

This paper uses data from the National Educational Panel Study (NEPS): Starting Cohort 4 - 9th Grade, doi:10.5157/NEPS:SC4:10.0.0. From 2008 to 2013, NEPS data were collected as part of the Framework Programme for the Promotion of Empirical Educational Research funded by the German Federal Ministry of Education and Research (BMBF). As of 2014, the NEPS survey is carried out by the Leibniz Institute for Educational Trajectories (LIfBi) at the University of Bamberg in cooperation with a nationwide network. 


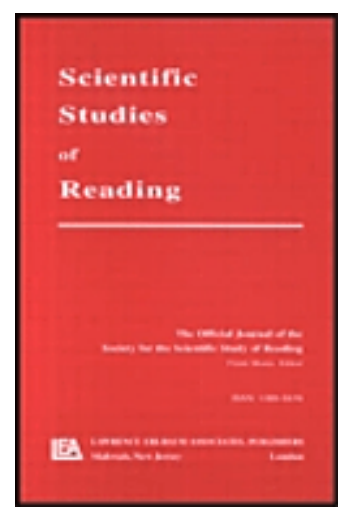

When do gender differences in reading competence diminish? A longitudinal study of women and men from adolescence to young adulthood.

\begin{tabular}{|r|l|}
\hline Journal: & Scientific Studies of Reading \\
\hline Manuscript ID & Draft \\
\hline Manuscript Type: & Research Article \\
\hline Keywords: & reading comprehension, gender, adolescents \\
\hline \multicolumn{2}{|l}{} \\
\end{tabular}

\section{SCHOLARONE ${ }^{\mathrm{m}}$ \\ Manuscripts}




\title{
When do gender differences in reading competence diminish? A longitudinal study of women and men from adolescence to young adulthood
}

\begin{abstract}
Various studies have revealed that girls perform better in reading than boys throughout school. However, there are also indications that gender differences change over time and diminish in young adulthood. The present study investigated the development of gender differences in reading competence from secondary school to after schooling during young adulthood in a longitudinal study to answer the research question whether and at what age gender differences diminish or disappear. The sample was part of the German National Educational Panel Study (NEPS), which consisted of 14,502 participants approximately 16 years old at the first measurement point. The results of a two group neighbor change model revealed gender differences in reading competence between young women and men at age 16 and at age 18 . However, the difference disappeared by age 22. As expected, young men's and women's reading competence developed in a differential and more linear fashion. Men had lower initial reading competence but a higher increase over time than women. Conversely, women's reading competence plateaued at age 18 . The study indicated thus compensatory growth in men's reading competence compared to women. The results are discussed in relation to previous findings concerning gender differences in reading.
\end{abstract}


Reading competence is a core prerequisite for learning across the lifespan and for societal participation. Moreover, reading competence is a key competence for active and successful participation in various fields of education, work, and everyday life (Graesser, Singer, \& Trabasso, 1994; Mullis, Martin, Foy, \& Hopper, 2017; OECD, 2013, OECD, 2016a, OECD, 2019). Basic reading skills are acquired at the beginning of formal schooling. However, reading competence involves far more than the successful acquisition of reading skills. While reading instruction is no longer a distinct subject in secondary school, reading remains a necessary skill for understanding written material. Therefore, 'reading to learn' is practiced and strengthened throughout students' school careers and beyond (Alexander, 2005). Consequently, it is relevant to consider developmental patterns of reading competence in students' later school years as well as after graduation.

Gender is an individual characteristic that has been empirically proven to be related to reading competence. Even though in several psychological variables women and men in general are similar (gender similarities hypothesis; Hyde, 2005, 2014) there are consistent findings of gender differences in reading competence in primary and secondary school years. Various studies have shown that girls in general outperform boys in reading competence throughout school (e.g., Berendes et al., 2018; Cameron, Grimm, Steele, Castro-Schilo, \& Grissmer, 2015; Mullis et al., 2017; OECD, 2016a, OECD, 2019; Solheim \& Lundetræ, 2017; Reilly, Neumann, \& Andrews, 2018). Previous research further indicates a growing gender difference in reading competence from primary school to secondary school, followed by a decrease from the end of secondary school to young adulthood (OECD, 2016b; Wicht, Rammstedt, \& Lechner, 2020). These findings suggest that gender differences in reading competence change over time and diminish in adulthood. However, most of these results are not based on longitudinal studies and therefore rely on different 
samples and differing ways of measuring reading competence. These different operationalization in turn impede the comparability of existing studies and their respective findings.

Summarizing previous studies, there is a lack of longitudinal research on the development of reading competence after students graduate from secondary school. This research gap refers not only to how reading competence develops after age 17 in general, but also to how female and male readers' competences develop in adulthood. Existing studies considering gender differences in reading competence from a longitudinal perspective mostly cease to measure reading competence at the latest with the end of secondary school (De Fraine, Van Damme, \& Onghena, 2007; Nagy, Reilly et al., 2018; Retelsdorf, Goldhammer, Schiepe-Tiska, \& Lüdtke, 2017).

Therefore, the aim of the present study was to describe the development of gender differences in reading competence at the end of secondary school and to answer the research question suggested by previous, mostly cross-sectional studies of whether and to what extent gender differences diminish or disappear during the transition from secondary school to young adulthood. Reading competence was measured in a sample of German young people at three points in time: at ages 16, 18, and 22. The development of men's and women's reading competence was longitudinally analyzed using a neighbor change model (McArdle, 2008; Steyer, Partchev, \& Shanahan, 2000). With this model, we investigate reading differences between the three time points without assuming a functional form of the change over time. In addition, it is straightforward to incorporate explanatory variables for the longitudinal change in reading competence. As such, the impact of a set of background variable was analyzed by comparing the observed and the covariate adjusted gender differences. 


\section{Development of reading competence}

The current paper employs a definition of reading competence based on a functional understanding of reading, similar to that taken in the OECD's (Organisation for Economic Co-operation and Development) reading literacy framework (cf. OECD, 1999). Reading competence is also often defined as engaging with the content of a text and obtaining information, drawing inferences and placing the text in a globally coherent structure (Graesser et al., 1994). Consequently, reading competence is determined as involving more than the mere decoding of letters, words, and sentences. Kintsch (1998) emphasizes the reader's active role during reading, which involves integrating previous knowledge with a mental representation of the text.

A lengthy and complex process encompassing psycholinguistic, perceptual, cognitive skills, and social exchange is required to obtain a functional understanding of reading competence (Gee, 2001). Children learning to read German usually read systematically in their third year of schooling, when they progress from the alphabetic to the orthographic stage of reading (Becker, McElvany, \& Kortenbruck, 2010; Scarborough, 2002). Reading competence and reading competence instruction change between primary school and secondary school. In children's first years of school, it is important to teach basic reading competence so that they can learn to read (e.g., Becker et al., 2010; Authors, 2013a). Afterwards, the focus is more on reading to learn, which involves more complex processes of understanding and competently handling texts (Becker et al., 2010; Authors, 2013a). This highlights the lifelong importance of reading, as processes involved in improving specific aspects of reading (e.g., drawing inferences from texts, readers' prior knowledge, and interest in reading) and thus enhancing general reading do not stop with schooling, but also remain relevant in adulthood (Alexander, 2005). 
Retelsdorf, Köller, and Möller (2011) uncovered an additional increase in reading competence after German children's transition to secondary school, between Grades 5 and 8 . The average growth in reading comprehension for all students over these three years had an effect size of $d=0.85$. However, this growth in reading competence was not linear, but decelerated towards the end of the period under investigation. Moreover, while several studies had examined reading competence development in primary school and until the eighth grade (e.g., Berendes et al., 2018; Niklas \& Schneider, 2012; Retelsdorf et al., 2011; Robinson \& Lubienski, 2011), only a few studies have investigated reading competence at end of secondary school. For example, Nagy and colleagues (2017) examined the development of reading competence in Grades 9 and 10; they found constant competence levels, but no increases in reading proficiency among the overall sample. To our knowledge, no longitudinal studies have examined the development of reading competence during the transition from secondary school to young adulthood.

While studies examining the development of reading competence have generally found an increase in students' average competence levels at least until the middle of secondary school (e.g., Retelsdorf et al., 2011), this does not necessarily apply to all specific subgroups of students. Three different theoretical models for the development of reading competence among different groups of students are possible: First, uniform growth in competences would mean the differences between groups remain the same over time. Second, the competence differences between groups can increase over time (i.e., the Matthew effect). And third, the difference between groups could decrease over time as one group makes up their earlier disadvantage (Berendes et al., 2018; Authors, 2014). 


\section{A longitudinal perspective on gender differences in reading}

As previously mentioned, reading competence is a prerequisite for active and successful learning and societal participation across the lifespan. However, although there are many similarities between women and men (Hyde, 2005, 2014), previous studies indicate that girls and boys have in general different levels of reading competence.

Most existing studies investigate gender differences at the beginning of primary school. Cameron and colleagues (2015) as well as Robinson and Lubienski (2011) found that girls generally reach higher reading levels than boys during their first years of school.

PIRLS (Progress in International Reading Literacy Study) investigated the reading competence of students in Grade 4. The overall trend for all previous PIRLS surveys revealed that girls have higher reading competence than boys on average in almost all participating countries (Mullis et al., 2017). In the PIRLS 2016, the OECD average reading competence was $M=520$ points for girls $(S D=0.4)$ and $M=501$ points for boys $(S D=0.5)$ (Mullis et al., 2017).

Berendes and colleagues (2018) examined German students between Grades 5 and 8, finding that boys had lower initial reading competence at the beginning of secondary school than girls. Moreover, boys experienced less growth in reading competence over subsequent school years than girls. Thus, the results indicated cumulative growth to the disadvantage of boys in reading competence.

PISA (Programme for International Student Assessment) results with respect to gender differences in reading competence among adolescents are similar to those of other studies with younger age groups; however, the extent of differences in reading competence between boys and girls was even higher. In all seven previous PISA cycles, 15-year-old girls achieved significantly higher reading competence scores than their male peers (OECD, 2019). In 2018, girls performed 
on average 30 points better than boys in the reading competence assessment across all OECD countries (girls: $M=502$ points, $S D=0.5$; boys: $M=472$ points, $S D=0.6$; OECD, 2019).

Only a few studies on gender differences in reading competence have examined the end of secondary school or even beyond in tertiary education and adulthood. Nagy and colleagues (2017) examined the development of reading competence in Grades 9 and 10. They found a small performance advantage for girls compared to boys at both measurement points.

De Fraine and colleagues (2007) investigated gender differences in language achievement (curriculum-relevant multiple-choice tests in grammar, reading comprehension, and spelling) in Dutch between Grades 7 and 12, finding evidence of differential development in girls' and boys' language achievement. It should be noted that this study's language achievement test measured more than just reading competence. Nevertheless, De Fraine and colleagues (2007) found differential growth patterns among boys and girls. Girls experienced constant growth in language achievement and therefore achieved a substantial learning gain at the end of secondary school compared to the start. However, for boys the pattern differed over time. Boys had higher language competences at Grade $7(M=3.53$ logits $)$ than girls $(M=3.47$ logits $)$, but then boys' language achievement declined until halfway through Grade 9, before subsequently increasing again until Grade 12. The combined IRT-scaled test in grammar, reading comprehension, and spelling also revealed higher initial competence for boys in Grade 7. Interestingly, De Fraine and colleagues (2007) showed that boys' language achievement dropped first before increasing again, and almost reached the same level as girls' achievement in twelfth grade. The authors explained the decrease of boys' achievement as due to their potential lack of test motivation at the end of Grade 8.

There are only a few studies on adults' reading competence, with less clear results on gender differences in reading abilities (OECD, 2016b; Solheim \& Lundetræ, 2017). The PIAAC study 
(Programme for the International Assessment of Adult Competencies) revealed no substantial gender differences for adults between 16 and 64 years old on average across OECD countries (OECD, 2016b).

Although variables to explain gender differences in reading competence were beyond the scope of the present study, potential mechanisms of the development and perpetuation should be described briefly. Partially but not only due to these competence differences, the domain of reading and associated activities are often seen as female-typed activities (e.g., Reilly et al., 2018; Watson, Kehler, \& Martino, 2010). Social expectations and attitudes can in turn influence interest and motivation for certain activities (cf. Eccles, 1987). Overall differences between boys and girls in reading motivation (Becker et al., 2010; Guthrie \& Wigfield, 2000; McElvany, Kortenbruck, \& Becker, 2008; Authors, 2015), reading behavior (Authors, 2013b), attitudes toward reading (Logan \& Johnston, 2009; Schiefele, Schaffner, Möller, \& Wigfield, 2012), and reading amount (Schaffner, Philipp, \& Schiefele, 2016) are the mechanisms that contribute most to explaining differences in reading competence. Girls' attitudes towards reading have been shown to be more positive than boys' attitudes (Logan \& Johnston, 2009; McGeown, Norgate, \& Warhurst, 2012; Wigfield, Gladstone, \& Turci, 2016). Studies show that girls also generally possess higher levels of intrinsic reading motivation and read more frequently on average (Logan \& Johnston, 2009; McGeown et al., 2012; Schiefele et al., 2012; Authors, 2015). These differences in reading motivation, attitudes and frequency can lead to differences in reading competence between girls and boys. 


\section{Purpose in the present study}

Against the critical importance of reading for lifelong learning and the empirical background, the present study focuses on the longitudinal development of reading. The present study has the priority to describe the gender gap between young women and men in reading at an important life transition for young people from secondary school into the labor market or tertiary education. Therefore, this study contributes to the field of longitudinal reading research and extended previous research on gender differences in reading competence of younger participants (Cameron et al., 2015; De Fraine et al., 2007; Nagy et al., 2017; OECD, 2016a; Reilly et al., 2018). Consequently, so far no statements can be made about the development of reading competence for young adults in this important phase of life for young people.

In summary, previous studies have revealed that girls generally reach a higher level of reading competence earlier than boys and that these differences remain relatively stable throughout students' school years (Cameron et al., 2015; De Fraine et al., 2007; Nagy et al., 2017; Retelsdorf et al., 2011; Robinson \& Lubienski, 2011). Indeed, PISA results indicate the largest gender differences in reading between girls and boys in secondary school (OECD, 2016a). However, De Fraine and colleagues (2007) showed that girls' and boys' language achievement developed differently in secondary school, with boys being able to catch up to girls. Similarly, no substantial gender differences for adults between 16-64 years old were found (OECD, 2016b). Thus, there is evidence that gender differences in reading diminish by adulthood. Solheim and Lundetræ (2017) provided further support to this notion by comparing gender differences in PIRLS 2011 and PISA 2009 in the Nordic countries (Denmark, Finland, Norway, and Sweden) as well as among young participants (16-24 years old) in PIAAC 2012. They reported gender differences with small effect sizes $(d=.19$ to .33$)$ for PIRLS (students in Grade 4$)$, medium effect sizes $(d=.34$ to .64 ) for 
PISA (15-year-old students), and non-existent gender differences $(d=-.01$ to .12) for PIAAC (young adults between 16-24 years old).

Moreover, no longitudinal study has to our knowledge consistently measured reading competence beyond school, especially during the transition from school to young adulthood and other educational settings like university, vocational training, or further education. Hence, the present study investigates gender differences in reading competence using a longitudinal framework from the end of secondary school to young adulthood from age 16 until age 22 . The study's guiding research questions concerned the longitudinal pattern of gender differences in reading competence.

Therefore, the following hypotheses were examined in the present study:

1. We hypothesize that gender differences diminish over time. While gender differences in reading competence are expected in adolescence at Grade 9, differences between women and men are expected to diminish by young adulthood.

2. Accordingly, we expect differential changes in reading competence for women and men. Men are expected to catch up to women's reading competence level in young adulthood at the latest.

\section{Method}

The study was conducted with data from the German National Educational Panel Study ${ }^{1}$ (NEPS). NEPS is a longitudinal study on educational trajectories and outcomes through the life course in Germany (Blossfeld, Roßbach, \& von Maurice, 2011). The study's multi-cohort sequence design

\footnotetext{
${ }^{1}$ All procedures and instruments are carefully checked by the NEPS data protection team and follow professional guidelines and ethical standards. Moreover, the Federal Ministries of Education in Germany approved the study.
} 
makes it possible to examine the trajectories of different age groups. This study used data from NEPS Starting Cohort 4, which began with German students in Grade 9. The students were recruited and surveyed in fall/winter 2010 (T1). At the beginning of the study, all data were collected from the students at school. Students who left school after ninth or tenth grade were individually interviewed at home during the second and third measurement points.

In addition to survey questionnaires, which were conducted at all four measurement points (T1 at the beginning of Grade 9), reading competence was measured at T2 (wave 2, spring 2011, school setting, end of Grade 9), at T3 (wave 7, 2013/2014, students in school setting in Grade 12 and participants not attending school were measured in an individual setting at home) and at T4 (wave 10, 2016/2017, all students in an individual setting at home). All measurements were carried out according to standardized guidelines and proctored by trained interviewers. At T1 (wave 1, fall/winter 2010, school setting, beginning of Grade 9), information on the participants' gender and the control variables (e.g., basic cognitive skills, migration background) were gathered. The design of the reading competence assessment at the different measurement points is illustrated in Table 1.

insert Table 1 -----

\section{Sample}

The final sample for this study contained 14,502 students ${ }^{2}$ enrolled in Grade 9 in Germany (7,226 female, $49.8 \%)$. At T2, the mean age was 15.72 years $(S D=.72$, range from $12-19$ years $)$. For

\footnotetext{
${ }^{2}$ All analyses were replicated for different groups of participation: $n=3,289$ students participated in all measurement points; $n=2,674$ students participated only in T2. The pattern of results showed in general no differences from the reported results for gender differences in reading. The sample description and results of the additional analyses presented in online supplemental material. https://osf.io/gw749/?view_only=974862517d9b41508dcb7ca16f230fb1
} 
3,595 students $(24.8 \%)$, at least one family member (participant, mother, or father) was not born in Germany. 126 participants $(0.9 \%)$ did not specify their country of birth.

In Grade 9, 5,356 students (36.9\%) in the sample attended an academic-track school, i.e., Gymnasium. The other 9,146 students attended a general secondary school or intermediate secondary school and were grouped together. The overall descriptive information of the sample as well as the descriptive information separate for women and men are depicted in Table 2. insert Table 2 -----

\section{Instruments}

Reading competence. The NEPS reading competence assessment was based on a theoretical framework considering prominent models of text comprehension (see Authors, 2013c) and is understood as a product of the reading process. The reading competence test was conducted of five different types of texts (informational, literary, instructional, argumentative, and advertising texts) in continuous format and focused on realistic, age-specific themes (Authors, 2013c). It involved different task types (dichotomous multiple choice items and complex polytomous multiple-choice items such as ordering and cloze tasks) with different cognitive requirements for the reader: finding information in the text, drawing text-related conclusions (local and global coherence formation), and reflection and assessment (situation model) (Authors, 2013c).

The duration of the reading competence assessment at all measurement points was 28 minutes. At each measurement point a new reading competence test was developed to avoid memory effects with new texts and tasks appropriate for the respective age group. Therefore, the number of tasks varied in each measurement point (cf. Table 1). The longitudinal assessment took the form of 
GENDER DIFFERENCES IN READING COMPETENCE FROM ADOLESCENCE TO YOUNG ADULTHOOD

tailored testing during the later measurement points: students were assigned to either an easy or difficult test booklet at measurement points T3 and T4 based on their previous performance on the test.

Reading competence was assessed with a paper-and-pencil-test at two measurement points (T2 and T3). During the last measurement point (T4), the assessment mode shifted to computer-based assessment (cf. Table 1). The overall effect of the different modes was simultaneously examined in a linking study for the longitudinal assessment (Fischer, Rohm, Gnambs, \& Carstensen, 2016). However, a potential differential impact of this change in assessment mode by gender has not yet been examined. Therefore, a potential interaction between gender and a mode effect on this study's findings will be discussed in more detail in the discussion section.

Reading competence scores were scaled in a partial credit IRT model for each reading competence test by using the dichotomous and polytomous test items (cf. Pohl \& Carstensen, 2012, for more information on scaling in NEPS). The test's fairness along several dimensions, including gender, was evaluated via a differential item functioning (DIF) analysis. At all measurement points, the DIF analyses confirmed measurement invariance for women and men (Gnambs, Fischer, \& Rohm, 2017; Haberkorn, Pohl, Hardt, \& Wiegand, 2012). Furthermore, to ensure longitudinal measurement invariance and to allow for comparisons of competence levels at different measurement points (i.e., examining the development over time), the reading competence measures at different measurement points were longitudinally linked using a mean/mean linking procedure with anchor items (Fischer et al., 2016). Based on the linked ITR models, each participant's reading competence was estimated as a weighted maximum likelihood estimate (WLE). These WLEs will be used as manifest indicators of reading competence for our longitudinal analysis on gender differences. 
Table 1 depicts the valid WLEs, revealing a large variation in the number of cases due to different reasons. On the one hand, a substantial drop out over time is present. This is plausible given the higher efforts for assessments beyond the school setting. On the other hand, a high proportion of design based missing are present at T3. Participants who were tested in an individual setting rather than a group setting in school were divided into different conditions (e.g., telephone interview without competence measurement or face-to-face interviews including competence assessments in reading, mathematics, and information and communication technology). Participants were randomly assigned to the telephone interview or face-to-face interview. Thus, missing competence measures in the telephone interview are at random due to the study design and, thus, do not systematically bias the test conditions.

Gender. An enrollment list provided by the school at the first measurement point T1 provided the students' gender as a dichotomous category, which was coded 0 for women and 1 for men.

Students' background. In this study, we controlled students' reading competence for a variety of background variables. Students' sociodemographic characteristics (socioeconomic status, migration background, and school type) and cognitive characteristics (cognitive basic skills) were assessed at $\mathrm{T} 1$.

Socioeconomic status, migration background, and school type. Parents' level of education and occupational status have an impact on children's reading ability and educational outcomes (Becker et al., 2010; Retelsdorf et al., 2011; Berendes et al., 2018; Cameron et al., 2015; Nagy et al., 2017). Students' socioeconomic background was measured by their parents' highest level of education. Data (reported by students) was available on 9,608 parents $(66.3 \%$; missing values for 4,894 
parents, 33.7\%). One-third of all parents had a university entrance qualification or higher $(4,570$, $31.5 \%$; i.e., Abitur), while another third had a non-academic educational qualification $(5,038$, $34.7 \%$; i.e., general secondary education leaving certificate). The descriptive for the overall sample as well as separately for women and men are depicted in Table 2.

Similarly, students with a migration background have been found to have lower levels of reading competence compared to students without a migration background. These differences in reading remained stable during secondary school (Berendes et al., 2018; Cameron et al., 2015; Nagy et al., 2017). In the present study, migration background was measured at T1 and defined as the students themselves or at least one of their parents having been born abroad. As aforementioned, 3,595 students $(24.8 \%)$ in the sample had a migration background, with at least one member of the core family (participant, mother, or father) having been born abroad. 126 participants $(0.9 \%)$ did not indicate their country of birth.

The school system in Germany is highly selective and encompasses different school types. Several studies have indicated that performance differences between students attending different school types increase over time (Retelsdorf, Becker, Köller, \& Möller, 2012; Retelsdorf \& Möller, 2008). For this reason, we also controlled for the students' school type. Students' school type was indicated by their teachers at $\mathrm{T} 1$. We grouped the results into academic-track schools (Gymnasium) which prepare students for higher education (5,356 students, 36.9\%), and nonacademic-track schools which emphasize non-university vocational training $(9,146$ students, $63.1 \%)$

Basic cognitive skills. In addition to sociodemographic characteristics, basic cognitive skills (e.g., working memory, decoding ability) have an impact on reading competence (McElvany \& Becker, 2010). In this study basic cognitive skills were controlled in the analyses and were measured by a 
test of reasoning ability, which was developed as a matrices test for NEPS. Analogous to RAVENmatrices (Raven, 1977), by comparison of eight alternative geometrical elements the correct inference must be drawn using logical rules (Haberkorn \& Pohl, 2013; Lang, Kamin, Rohr, Stünkel, \& Williger, 2014). The sum scores for all participants at age 16 averaged $M=8.66$ $(S D=2.46 ; \operatorname{Min}=$ no correct answer, Max $=12$ correct answers, Cronbach's $\alpha=0.67)$. For 613 participants (4.2\%), information on basic cognitive skills was not obtained.

\section{Statistical data analysis}

The longitudinal analysis of reading competence over the three measurement points and the differential development in men's and women's reading competence was conducted using a twogroup structural equation model with change variables (see e.g., the neighbor change model of Steyer, Eid, \& Schwenkmezger, 1997; Steyer et al., 2000, and difference score model of McArdle, 2008). The change variables represent the differences in reading competence between consecutive time points and, thus, allow for comparing possible non-linear development over time between the gender groups. Reading competence was included as manifest variable (WLE estimates) in the neighbor change model since the latent modeling of the competence, the test of measurement invariance (DIF) and the longitudinal linking for growth investigations was already conducted in the IRT-scaling approach (Pohl \& Carstensen, 2012).

Figure 1 is a schematic depiction of the neighbor change model in our application. The model reorganizes the information of the time point specific measures into new variables without any restrictions, enabling for interpretations and parameter tests on the new variables. For this, the three time point specific measures on reading competence are described with a baseline variable 
GENDER DIFFERENCES IN READING COMPETENCE FROM ADOLESCENCE TO YOUNG ADULTHOOD

and two change variables, whereas all loadings were fixed to 1, and all intercepts as well as error variances were fixed to zero, such as:

reading competence $\mathrm{T} 2($ age 16$)=0+1 *$ baseline

reading competence $\mathrm{T} 3($ age 18$)=0+1 *$ baseline $+1 *$ change 1 T3-T2

reading competence $\mathrm{T} 4($ age 22$)=0+1 *$ baseline $+1 *$ change $1_{\mathrm{T} 3-\mathrm{T} 2}+1 *$ change 2 T4-T3

\begin{abstract}
Alongside baseline reading competence, which describes the reading competence of young women and men at age 16, the two change variables describe the individual reading developments in comparison to the previous time point, (i.e., the individual changes in the reading competence between age 16 and 18 and age 18 and 22, respectively). In the two-group model for young women and young men, the change variables represent the gender-specific trajectories in reading competence.
\end{abstract}

Two models were estimated: the first model was the presented neighbor change model without control variables. The second model extends the first one to control for other variables, which potentially influence the reading competence of young women and men. For this aim, the control variables (socioeconomic status, migration background, school type, and basic cognitive skills) were included as explanatory variables for the baseline of reading competence and the two change variables. In order to rule out a different impact of the control variables for women and men (i.e., interaction effects of the control variables with gender), the slopes of the covariates on the baseline and each change variable, were constraint to be equal for women and men. This constraint did not 
result in a significant deterioration of model fit in the large sample (Wald Test of Parameter Constraints $(d f 12)=10.39, p=.58)$.

Finally, in order to be able to make a prediction about whether the expected gender differences in reading competence are considerable, we followed the framework of Mayer and colleagues (2016) and defined three new model parameters, which were tested for their significance (Mayer, Dietzfelbinger, Rosseel, \& Steyer, 2016). The first new model parameter defines the difference between young women and young men in the intercept of the baseline variable, and accordingly represents the comparison of the gender specific average reading competence at age 16 . The same difference was specified for the intercepts of the first and second change variable, which accordingly represents the comparison of the gender specific average change in reading competence between two consecutive measurement points. In the model with control variables and under the assumption of no interaction effects, the new parameters represent the covariate-adjusted average difference between men and women, respectively in the baseline competence or change. This allows for studying the impact of the control variables on the gender differences in the development of reading competence.

The model was estimated with the software Mplus (version 8; Muthén \& Muthén, 2017). The Mplus syntax, model output and additional missing analyses are available at: https://osf.io/gw749/?view_only=974862517d9b41508dcb7ca16f230fb1

For all models a full information maximum likelihood (FIML) estimator was used to handle missing values in the variables (Allison, 2009; Enders \& Bandalos, 2001). As already described, a rather high proportion of missing data was due to the survey design with randomly assigned test settings. Therefore, it can be assumed that this kind of missing data were completely at random. Other non-responses can be more systematic and, in this case, the application of FIML assumes 
that missing are conditional at random given the other variables in the analysis model (i.e., the observed reading competence at other time points, gender and, if present, the additional covariates). Zinn and Gnambs (2018) showed with the same data set that a FIML approach can adequately estimate even a large number of missing values. For validating the results regarding missing data, they suggest sensitivity analyses. Accordingly, the robustness of the subsequent results was checked for subgroups with more strict criteria on the observed data (i.e., either participation at all three measurement points was required, or participation at T2 and T3 - in comparison to using participants with observed data on at least one measurement point). The findings regarding gender differences in reading competence were comparable in the sensitivity analysis. All outputs are available at the aforementioned OSF project.

\title{
Results
}

\section{Descriptive statistics}

Table 3 shows the bivariate correlations among all variables in the final neighbor change model for young women and young men.

----- insert Table 3 ------

\begin{abstract}
Almost all variables were significantly correlated, with relatively stable and high positive correlations between reading competence at the different measurement points for young women $(r$ between .61 and .66) and young men ( $r$ between .58 and .66). Similarly, strong correlations between reading competence and the control variables were evident for young women and young
\end{abstract}


men. In particular, the correlations between basic cognitive skills (reasoning; $r$ between .40 and .48 ) and school type ( $r$ between -.42 and -.53) with reading competence were relatively stable over time and comparable between young women and young men. In addition, somewhat lower correlations between young women's and young men's reading competence and parents' level of education ( $r$ between .23 and .28 ) and migration background ( $r$ between -.17 and -.22) were found.

There were no significant gender differences in terms of the control variables concerning socioeconomic status and migration background. Young women and men were comparable with regard to their socioeconomic status $\left(\chi^{2}(1,9,608)=2.07, p=.15\right)$ and migration background $\left(\chi^{2}(1\right.$, $14,376)=1.98, p=.16)$.

However, the distribution of school types differed significantly between young women and young men $\left(\chi^{2}(1,14,336)=80.43, p=.00\right)$. At T1, more young women than young men attended academic-track schools (women: 2,932; men: 2,424).

Young men exhibited a significant, but minor advantage in basic cognitive skills $(t(13,887)=$ $2.56, p=.007 d=-.05)$. Young men had slightly higher scores in basic cognitive skills on average $(M=8.71, S D=2.46)$ compared to young women $(M=8.60, S D=2.46)$.

\section{Neighbor change models for gender differences in reading competence}

The results for the comparison of reading competence between women and men according to the two neighbor change models are depicted in table 4. Model 1 was computed without control variables and in model 2 the baseline and change variables in reading competence were adjusted for relevant background variables of the participants. Moreover, the differences in the average reading competence at age 16 (baseline) and the average changes were tested for their significance. 
Since the results did not change under control of the covariates (basic cognitive skills, parents' highest level of education, migration background, and school type) the interpretation of the results refer to model 1 without control variables. The selected control variables did primarily explain variances in reading competence at age 16 (explained variance women $\mathrm{R}^{2} 37 \%$, explained variance men $\mathrm{R}^{2} 33 \%$ ). However, the covariates appeared to be less important for the development of reading competence and did not predict the changes (change 1 : explained variance women $\mathrm{R}^{2} 3 \%$, explained variance men $\mathrm{R}^{2} 2 \%$; change 2 : explained variance women $\mathrm{R}^{2} 0.8 \%$, explained variance men $\left.\mathrm{R}^{2} 0.8 \%\right)$

Figure 2 illustrates the development of reading competence for young women and young men. The depicted slopes in figure 2 were based on the results of model 1 without control variables.

As expected, there was a gender difference in reading competence between women and men at T2: At age 16 , women had higher average reading competence $(M=0.14, S D=1.24)$ than men $(M=-0.19, S D=1.27)$. The comparison of the gender specific average at age 16 accounted for a difference of 0.33 logits $(S E=0.02)$ which corresponds to a significant small effect of $d=-.26$. This gender difference was also present at T3. Moreover, continued growth in reading competence from age 16 to age 18 was experienced by both women $(M=0.46, S D=0.96)$ and men $(M=0.24$, $S D=1.03)$. The comparison of the average in reading competence between young women and men at the ages of 18 showed also a small effect of $0.22 \operatorname{logits}(S E=0.02), d=-.22$. At the last measurement point in young adulthood (T4, age 22), there was no gender difference in reading competence between women and men; difference of 0.00 logits $(S E=0.02)$. Women and men had comparable reading competence (women: $M=0.43, S D=0.82$; men: $M=0.43, S D=0.83$ ).

In conclusion, Hypothesis 1 was supported by the data. There are gender differences in reading competence during students' school years, but no differences between women and men in young 
adulthood. Thus, it was confirmed that gender differences in reading competence diminish over time.

\section{------ insert Figure 2 -------}

Table 4 illustrates the results of the neighbor change model examining differential changes in reading competence among women and men. In addition to mean levels of reading competence, gender specific average changes between the measurement points for women and men are shown.

As depicted in figure 2, women had a higher reading competence at age 16 than men. Women's reading competence grew on average by 0.32 logits $(S E=0.02)$ between Grade 9 and the end of school-based education or vocational training at age 18. Men's reading competence increased on average by 0.43 logits $(S E=0.02)$ over the first two years of the study from Grade 9 on. There was a significant gender difference in the changes between age 16 and age 18 (-0.10 logits, $S E=0.02$ ), but the change in reading competence was smaller for women. However, on average women's reading competence did not develop significantly further between the ages of 18 and $22(-0.04$ logits, $S E=0.02)$. Hence, women's reading competence increased between ages 16 and 18 , yet, their reading competence remained stable towards the consecutive measurement point at the age of 22. Men's reading competence, however, further increased between the ages of 18 and 22 by 0.19 logits on average $(S E=0.02)$. This result is also reflected in the difference in changes between young women and young men from T3 to T4. The difference coefficient shows a significant change in reading competence in favor of young men $(-0.23$ logits; $S E=0.03)$ and the change of reading competence of young women at the last two measurement points is lower than between $\mathrm{T} 2$ and $\mathrm{T} 3$. 
GENDER DIFFERENCES IN READING COMPETENCE FROM ADOLESCENCE TO YOUNG ADULTHOOD

As expected in Hypothesis 2, there was a differential change in young men's reading competence. Men had lower initial reading competence at T2, but experienced a stronger and more linear increase in reading competence, compared to women's nonlinear and relatively stagnant trajectory after secondary school completion. insert Table 4

The correlation between reading competence at baseline and change 1 (T3-T2) was $r=-.61$ for women and $r=-.68$ for men. This result indicates that individuals with lower initial reading competence at baseline experienced a greater increase to T3. This gain was slightly higher for men than for women. The correlation between change 1 (T3-T2) and change 2 (T4-T3) was $r=-.28$ for women and $r=-.31$ for men. These lower correlations indicate that growth in reading competence decelerated over time.

In addition to the development of reading competence the standard deviations (also displayed in figure 2) highlight the variations within the groups. At age 16 both gender groups were dispersed about more than $1 \operatorname{logit}\left(S D_{\text {women }}=1.24, S D_{\text {men }}=1.27\right)$ which indicates a strong variation of reading competence within the groups. This heterogeneity in reading competence decreased over time for young women and young men. Both young women and young men became more similar in their reading competence $\left(S D_{\text {women }}=0.82, S D_{\text {men }}=0.83\right)$. 
GENDER DIFFERENCES IN READING COMPETENCE FROM ADOLESCENCE TO YOUNG ADULTHOOD

\section{Discussion}

The main goal of this study was to describe the longitudinal development of reading competence during the transition from secondary school to young adulthood. Therefore, the study aimed to investigate the gender gap in reading competence between young women and men from age 16 to age 22. This study extended previous research on gender differences in reading competence (Cameron et al., 2015; De Fraine et al., 2007; Nagy et al., 2017; OECD, 2016a; Reilly et al., 2018) by examining a longer period of time that included students' transition following secondary school completion. A further advantage of this study is its coherent framework for measuring reading competence (Authors, 2013c) across all three measurement points, and the longitudinal linking of competence data (Fischer et al., 2016) to actually display individual competence development.

As expected in Hypothesis 1, gender differences in reading competence diminished over time. The results of this study thus confirmed previous research regarding gender differences in reading competence in different age groups. Similar to the results of previous school studies (Berendes et al., 2018; Cameron et al., 2015; De Fraine et al., 2007; Nagy et al., 2017; OECD, 2016a; OECD, 2019), our study found evidence of gender differences in young women's and men's initial reading competence at the end of Grade 9 (around age 16). However, these differences were also found to diminish over time, with women and men exhibiting comparable reading competence in young adulthood (age 22). These results are in line with the results for young participants (aged 16-24) in the PIAAC study (OECD, 2016b). In contrast to the results of longitudinal studies with younger students (Grades 5 to 8: Berendes et al., 2018; Grades 9 to 10: Nagy et al., 2017), our study found evidence of compensatory growth in reading competence among men compared to women. 
Consequently, there was a differential change in women's and men's reading competence, in support of Hypothesis 2. In line with expectations, young men's reading competence was lower at the end of Grade 9 (age 16). However, young men caught up to women in terms of reading competence as a result of their constant increase in reading competence from age 16 until 22 . This result is in line with the findings of De Fraine and colleagues (2007), who showed that boys' achievement in reading dropped between Grades 7 and 9 before rising again, and almost reached the same level as girls' achievement in the twelfth grade. In the present study, young men experienced a greater and more linear increase in reading competence than young women. In contrast, young women experienced an increase in reading competence between ages 16 and 18 , but almost no change in reading competence between ages 18 and 22 . Women reached a plateau in reading competence at age 18 , which then remained constant at least until the age of 22. Yet, young men considerably increased their reading competence between ages 18 and 22 .

Various aspects can be considered to explain the findings of the present study. However, at this point we can only provide assumptions about the mechanisms to explain the development of gender differences in reading competence since potential no explanatory variables such as reading motivation or reading interest (e.g., Becker et al., 2010, Logan \& Johnston, 2009; McGeown at al., 2012; Schiefele et al., 2012), for example, were so far not measured longitudinally in NEPS.

A possible explanation for these results concerning men's compensatory growth in reading competence might be a change in their habitual motivation and reading behavior following school graduation because learning opportunities become more self-selected. This could also go along with a change in personal goals. Previous studies have repeatedly found a lack of gender differences in reading competence among younger age groups when controlling for reading 
motivation (Becker et al., 2010; Schiefele et al., 2012; Stanat \& Kunter, 2002). Author and colleagues $(2017 ; 2018)$ found a shift in goal orientation among a sample of young adults during the transition from school to vocational training and university; specifically, a higher mastery goal orientation (developing of one's own competences and learning new things; cf. Senko \& Dawson, 2017; Wirthwein, Sparfeldt, Pinquart, Wegerer, \& Steinmayr, 2013) was found for school graduates than for students still in school (Authors, 2017; Authors, 2018). These results suggest that young adults are motivated to expand their skills and knowledge following school graduation. The increase in mastery goal orientation indicates a better match between external goals and one's own personal goals. This match could be linked to a motivation boost in self-selected learning opportunities and might be positively related to the young people's competence development. Hence, an increase in mastery goals (cf. Senko \& Dawson, 2017; Wirthwein et al., 2013) after school graduation could lead to a change in reading motivation, reading behavior, and reading amount, which could in turn have a positive impact on young women's and men's reading competence. As compared to school students, young adults should have more self-selected reading opportunities that are more relevant to their daily lives, which might be beneficial for reading motivation.

\section{Limitation}

The first limitation to mention at this point is missing data especially in the reading competence assessment due to the study's survey design, but also due to the longitudinal approach of the NEPS which often has to deal with substantial dropout over time. The randomly assigned study design for individual tracking resulted in around two-thirds of the overall missing data in the present study considered as missing completely at random. However, when dealing with repeated measures, it 
is not surprising for a certain number of participants to drop out of subsequent measurement points (one-thirds of missing data therefore considered at random or not at random; cf. Schafer \& Graham, 2002). To address missing data, a model-based missing information estimator was applied (i.e. full information maximum likelihood) with a considerable number of background variables chosen to compensate for the missing information in the data. In addition, the robustness of the results for participants with (more) complete observations on reading competence was investigated. The sensitivity analysis shows comparable gender differences and changes over time.

As already mentioned, the administration mode changed from paper-and-pencil assessment to computer-based assessment between the third and the forth measurement point. No information is currently available on the interaction between gender and assessment mode in NEPS reading assessments. However, findings regarding changes in administration mode are available for the PISA study. Detailed analyses of PISA 2018 revealed that computer-based reading is comparable to reading on paper. However, computer-based tasks were found to be more difficult (Goldhammer et al., 2019). In a study with data from PISA 2012, Kroehne, Hahnel and Goldhammer (2019) analyzed reading measurement invariance between girls and boys and again found no evidence of a mode effect conditional on gender. However, they confirmed the well-known gender difference in reading competence, which could not be ascribed to administration mode (Kroehne, Hahnel et al., 2019). Since these recent studies did not reveal an effect of assessment mode on gender differences in reading competence (Goldhammer et al., 2019; Kroehne, Buerger, Hahnel, \& Goldhammer, 2019; Kroehne, Hahnel et al., 2019), we would expect similar results for the NEPS reading assessments. Moreover, Kroehne and colleagues $(2019 ; 2019)$ used data from the German PISA 2012 study, which should be comparable to our NEPS sample in terms of age and degree of digitalization. However, we cannot completely rule out an interaction between gender and 
GENDER DIFFERENCES IN READING COMPETENCE FROM ADOLESCENCE TO YOUNG ADULTHOOD

assessment mode in the present study; thus, an additional validation study on gender-specific mode effects in the NEPS assessments would be an outlook for future research.

\section{Conclusion}

In conclusion, the present study investigated the development of gender differences in reading competence in young adulthood, finding that the difference in reading competence between young women and men was confirmed at the age of 16 , yet disappeared by age 22 . Relevant background variables for explaining variance in reading competence had no impact on the observed gender differences. Although the underlying mechanisms for the gender differences cannot be revealed, from our point of view this study is an important contribution to reading research as it expands previous results on the age range of young readers and focusses on a longitudinal perspective. The fact that differences in reading competence between women and men are no longer present in young adulthood should not be used as an argument for idleness. Reading competence is an important prerequisite for general learning in school; thus, deficits might impair achievement in various subject domains. The early and persistent disparities between boys and girls during their school careers (e.g., Berendes et al., 2018; Cameron et al., 2015; Mullis et al., 2017; Robinson \& Lubienski, 2011) should thus be actively addressed. Early sensitivity to gender differences in reading competence seems indispensable to help boys not fall behind girls in terms of reading competence in school and prevent them from needing to catch up later in life. 


\section{References}

Alexander, P. A. (2005). The path to competence: A lifespan developmental perspective on reading. Journal of Literacy Research, 37(4), 413-436. https://doi.org/10.1207/s15548430jlr3704_1

Allison, P. D. (2009). Missing data. Thousand Oaks, California: SAGE Publications.

Authors, 2013a

Authors, 2013b

Authors, 2013c

Authors, 2014

Authors, 2015

Authors, 2017

Authors, 2018

Becker, M., McElvany, N., \& Kortenbruck, M. (2010). Intrinsic and extrinsic reading motivation as predictors of reading literacy: A longitudinal study. Journal of Educational Psychology, 102(4), 773-785. https://doi.org/10.1037/a0020084

Berendes, K., Becker, M., Jacoby, J., Flunger, B., Nagengast, B., \& Trautwein, U. (2018). Individuelle Entwicklungsverläufe beim Lesen. Macht das Geschlecht den Unterschied? [Individual developmental progress in reading: Does gender make a difference?]. Zeitschrift für Entwicklungspsychologie und Pädagogische Psychologie, 50(4), 192-208. https://doi.org/10.1026/0049-8637/a000202

Blossfeld, H.-P., Roßbach, H.-G., \& von Maurice, J. (2011). Education as a lifelong process: The German National Educational Panel Study (NEPS). Zeitschrift für Erziehungswissenschaft/special issue: Vol. 14. Wiesbaden: VS Verlag für Sozialwissenschaften.

Cameron, C. E., Grimm, K. J., Steele, J. S., Castro-Schilo, L., \& Grissmer, D. W. (2015). Nonlinear gompertz curve models of achievement gaps in mathematics and reading. Journal of Educational Psychology, 107(3), 789-804. https://doi.org/10.1037/edu0000009

De Fraine, B., Van Damme, J., \& Onghena, P. (2007). A longitudinal analysis of gender differences in academic self-concept and language achievement: A multivariate multilevel latent growth approach. Contemporary Educational Psychology, 32(1), 132-150. https://doi.org/10.1016/j.cedpsych.2006.10.005

Eccles, J.S. (1987). Gender roles and women's achievement-related decisions. Psychology ofWomen Quarterly, 11(2), 135-172.

Enders, C. K., \& Bandalos, D. L. (2001). The relative performance of full information maximum likelihood estimation for missing data in structural equation models. Structural Equation Modeling: A Multidisciplinary Journal, 8(3), 430-457. https://doi.org/10.1207/S15328007SEM0803_5 
Fischer, L., Rohm, T., Gnambs, T., \& Carstensen, C. H. (2016). Linking the data of the competence tests (NEPS Survey Paper No. 1). Bamberg: Leibniz Institute for Educational Trajectories, National Educational Panel Study. Retrieved from https://www.nepsdata.de/Portals/0/Survey\%20Papers/SP_I.pdf

Gee, J. (2001). Reading as situated language: A sociocognitive perspective. Journal of Adolescent \& Adult Literacy, 44(8), 714-725. https://doi.org/10.1598/JAAL.44.8.3

Gnambs, T., Fischer, L., \& Rohm, T. (2017). NEPS technical report for reading: scaling results of starting cohort 4 for grade 12 (NEPS Survey Paper No. 13). Bamberg: Leibniz Institute for Educational Trajectories, National Educational Panel Study. Retrieved from https://www.neps-data.de/Portals/0/Survey\%20Papers/SP_XIII.pdf

Goldhammer, F., Harrison, S., Buerger, S., Kroehne, U., Lüdtke, O., Robitzsch, A., . . Mang, J. (2019). Vertiefende Analysen zur Umstellung des Modus von Papier auf Computer [Deepening analyzes to cutover the mode from paper to computer]. In K. Reiss, M. Weis, E. Klieme, \& O. Köller (Eds.), PISA 2018: Grundbildung im internationalen Vergleich (1st ed., pp. 163-186). Münster: Waxmann.

Graesser, A. C., Singer, M., \& Trabasso, T. (1994). Constructing inferences during narrative text comprehension. Psychological Review, 101(3), 371-395. https://doi.org/10.1037/0033295X.101.3.371

Guthrie, J. T., \& Wigfield, A. (2000). Engagement and motivation in reading. In M. L. Kamil, P. B. Mosenthal, P. D. Pearson, \& R. Barr (Eds.), Handbook of reading research (3rd ed., pp. 403-422). Mahwah, NJ: Lawrence Erlbaum Associates Publishers.

Haberkorn, K., \& Pohl, S. (2013). Cognitive basic skills (non-verbal): data in the scientific use file. Bamberg: University of Bamberg, National Educational Panel Study. Retrieved from https://www.nepsdata.de/Portals/0/NEPS/Datenzentrum/Forschungsdaten/SC3/com_cogbasic2013_en.pdf

Haberkorn, K., Pohl, S., Hardt, K., \& Wiegand, E. (2012). NEPS technical report for readingscaling results of starting cohort 4 in ninth grade (NEPS working paper No. 16). Bamberg: University of Bamberg, National Educational Panel Study. Retrieved from https://www.nepsdata.de/Portals/0/Working\%20Papers/WP_XVI.pdf

Hyde, J. S. (2005). The gender similarities hypothesis. American Psychologist, 60 (6), 581-592. https://doi.org/10.1037/0003-066X.60.6.581

Hyde, J. S. (2014). Gender similarities and differences. Annual Review and Psychology, 65, 373398. https://doi.org/10.1146/annurev-psych-010213-115057

Kintsch, W. (1998). Comprehension: A paradigm for cognition. Cambridge: Cambridge Univ. Press.

Kroehne, U., Buerger, S., Hahnel, C., \& Goldhammer, F. (2019). Construct equivalence of PISA reading comprehension measured with paper-based and computer-based assessments.

Educational Measurement: Issues and Practice, 31(1), 1-15.

https://doi.org/10.1111/emip.12280 
Kroehne, U., Hahnel, C., \& Goldhammer, F. (2019). Invariance of the response processes between gender and modes in an assessment of reading. Frontiers in Applied Mathematics and Statistics, 5, 2. https://doi.org/10.3389/fams.2019.00002

Lang, F. R., Kamin, S., Rohr, M. K., Stünkel, C., \& Williger, B. (2014). Erfassung der fluiden kognitiven Leistungsfähigkeit über die Lebensspanne im Rahmen der National Educational Panel Study: Abschlussbericht zu einer NEPS-Ergänzungsstudie[Capture of fluid cognitive performance over the lifespan as part of the National Educational Panel Study: final report on a NEPS supplementary study]. (NEPS working paper No. 43). Bamberg: Leibniz Institute for Educational Trajectories, National Educational Panel Study. Retrieved from https://www.neps-data.de/Portals/0/Working\%20Papers/WP_XLIII.pdf

Logan, S., \& Johnston, R. (2009). Gender differences in reading ability and attitudes: examining where these differences lie. Journal of Research in Reading, 32(2), 199-214. https://doi.org/10.1111/j.1467-9817.2008.01389.x

Mayer, A., Dietzfelbinger, L., Rosseel, Y., \& Steyer, R. (2016). The effectliteR approach for analyzing average and conditional effects. Multivariate Behavioral Research, 51(2-3), 374391. https://doi.org/10.1080/00273171.2016.1151334

McArdle, J. J. (2008). Latent variable modeling of differences and changes with longitudinal data. Annual Review of Psychology, 60(1), 577-605. https://doi.org/10.1146/annurev.psych.60.110707.163612

McElvany, N., \& Becker, M. (2010). Welche Prädiktoren braucht man zur Vorhersage von Lesekompetenz? Eine Kommunalitätenanalyse zur Bestimmung der uniquen und geteilten Varianzaufklärung psychologischer und soziologischer Konstrukte [What predictors do you need to predict reading competence? A community analysis to determine the unique and shared variance explanation of psychological and sociological constructs]. In W. Bos, E. Klieme, \& O. Köller (Eds.), Schulische Lerngelegenheiten und Kompetenzentwicklung: Festschrift für Jürgen Baumert (pp. 147-172). Münster: Waxmann.

McElvany, N., Kortenbruck, M., \& Becker, M. (2008). Lesekompetenz und Lesemotivation. Entwicklung und Mediation des Zusammenhangs durch Leseverhalten [Reading literacy and reading motivation: their development and the mediation of the relationship by reading behavior]. Zeitschrift für Pädagogische Psychologie, 22(3-4), 207-219. https://doi.org/10.1024/1010-0652.22.34.207

McGeown, S. P., Norgate, R., \& Warhurst, A. (2012). Exploring intrinsic and extrinsic reading motivation among very good and very poor readers. Educational Research, 54(3), 309-322. https://doi.org/10.1080/00131881.2012.710089

Mullis, I. V. S., Martin, M. O., Foy, P., \& Hopper, M. (2017). PIRLS 2016 international results in reading. Boston. Retrieved from http://timssandpirls.bc.edu/pirls2016/international-results/

Muthén, L. K., \& Muthén, B. O. (2017). Mplus user's guide (Eighth Edition). Los Angeles, CA: Muthén \& Muthén.

Nagy, G., Retelsdorf, J., Goldhammer, F., Schiepe-Tiska, A., \& Lüdtke, O. (2017). Veränderungen der Lesekompetenz von der 9. zur 10. Klasse: Differenzielle Entwicklungen in Abhängigkeit der Schulform, des Geschlechts und des soziodemografischen Hintergrunds? 
[Changes in reading skills from 9th to 10th grade: differential trajectories depending on school type, gender, and socio-demographic background?]. Zeitschrift für Erziehungswissenschaft, 20(2), 177-203. https://doi.org/10.1007/s11618-017-0747-1

Niklas, F., \& Schneider, W. (2012). Die Anfänge geschlechtsspezifischer Leistungsunterschiede in mathematischen und schriftsprachlichen Kompetenzen [The beginning of gender-based performance differences in mathematics and linguistic competencies]. Zeitschrift für Entwicklungspsychologie und Pädagogische Psychologie, 44(3), 123-138.

https://doi.org/10.1026/0049-8637/a000064

OECD (1999). Measuring student knowledge and skills: A new framework for assessment. Paris: OECD Publishing.

OECD (2013). The survey of adult skills: Reader's companion. Paris: OECD Publishing.

OECD (2016a). PISA 2015 results (Volume 1): Excellence and equity in education. PISA: Vol. 1. Paris: OECD Publishing.

OECD (2016b). Skills matter: Further results from the survey of adult skills. Paris: OECD Publishing.

OECD (2019). PISA 2018 Results: What Students Know and Can Do. Paris: OECD Publishing.

Pohl, S., \& Carstensen, C. H. (2012). NEPS technical report - scaling the data of the competence tests (NEPS working paper No. 14). Bamberg: University of Bamberg, National Educational Panel Study. Retrieved from https://www.nepsdata.de/Portals/0/Working\%20Papers/WP_XIV.pdf

Raven, J. C. (1977). Standard pogressive mtrices: Sets A, B, C, D\& E. San Antonio: Harcourt Assessment PsychCorp.

Reilly, D., Neumann, D. L., \& Andrews, G. (2018). Gender Differences in Reading and Writing Achievement: Evidence From the National Assessment of Educational Progress (NAEP). American Psychologist. Advance online publication.

http://dx.doi.org/10.1037/amp0000356

Retelsdorf, J., Becker, M., Köller, O., \& Möller, J. (2012). Reading development in a tracked school system: A longitudinal study over 3 years using propensity score matching. The British Journal of Educational Psychology, 82(Pt 4), 647-671. https://doi.org/10.1111/j.20448279.2011.02051.x

Retelsdorf, J., Köller, O., \& Möller, J. (2011). On the effects of motivation on reading performance growth in secondary school. Learning and Instruction, 21(4), 550-559. https://doi.org/10.1016/j.learninstruc.2010.11.001

Retelsdorf, J., \& Möller, J. (2008). Entwicklungen von Lesekompetenz und Lesemotivation. Schereneffekte in der Sekundarstufe? [Developments of reading literacy and reading motivation: Achievement gaps in secondary school?]. Zeitschrift für Entwicklungspsychologie und Pädagogische Psychologie, 40(4), 179-188. https://doi.org/10.1026/0049-8637.40.4.179

Robinson, J. P., \& Lubienski, S. T. (2011). The development of gender achievement gaps in mathematics and reading during elementary and middle school. American Educational Research Journal, 48(2), 268-302. https://doi.org/10.3102/0002831210372249 
Scarborough, H. S. (2002). Connecting early language and literacy to later reading (dis)abilities: Evidence, theory and practice. In S. B. Neuman \& D. K. Dickinson (Eds.), Handbook of Early Literacy Research (pp. 97-110). New York: Guilford Press.

Schafer, J. L., \& Graham, J. W. (2002). Missing data: Our view of the state of the art. Psychological Methods, 7(2), 147-177. https://doi.org/10.1037//1082-989X.7.2.147

Schaffner, E., Philipp, M., \& Schiefele, U. (2016). Reciprocal effects between intrinsic reading motivation and reading competence? A cross-lagged panel model for academic track and nonacademic track students. Journal of Research in Reading, 39(1), 19-36. https://doi.org/10.1111/1467-9817.12027

Schiefele, U., Schaffner, E., Möller, J., \& Wigfield, A. (2012). Dimensions of reading motivation and their relation to reading behavior and competence. Reading Research Quarterly, 47(4), 427-463. https://doi.org/10.1002/RRQ.030

Senko, C., \& Dawson, B. (2017). Performance-approach goal effects depend on how they are defined: Meta-analytic evidence from multiple educational outcomes. Journal of Educational Psychology, 109(4), 574-598. https://doi.org/10.1037/edu0000160

Solheim, O. J., \& Lundetræ, K. (2017). Can test construction account for varying gender differences in international reading achievement tests of children, adolescents and young adults? - A study based on Nordic results in PIRLS, PISA and PIAAC. Assessment in Education: Principles, Policy \& Practice, 3, 1-20. https://doi.org/10.1080/0969594X.2016.1239612

Stanat, P., \& Kunter, M. (2002). Geschlechterspezifische Leistungsunterschiede bei Fünfzehnjährigen im internationalen Vergleich [An international comparison of genderspecific differences in achievement of 15-year-olds]. Zeitschrift für Erziehungswissenschaft, 4(1), 28-48. https://doi.org/10.1007/s11618-002-0003-0

Steyer, R., Eid, M., \& Schwenkmezger, P. (1997). Modeling true intraindividual change: True change as a latent variable. Methods of Psychological Research, 2(1), 21-33.

Steyer, R., Partchev, I., \& Shanahan, M. J. (2000). Modeling true intraindividual change in structural equation models: The case of poverty and children's psychosocial adjustment. In T. D. Little, K. U. Schnabel, \& J. Baumert (Eds.), Modeling longitudinal and multilevel data: Practical issues, applied approaches, and specific examples (pp. 109-126). Hillsdale, New York: Erlbaum.

Watson, A., Kehler, M., \& Martino, W. (2010). The problem of boys' literacy underachievement. Raising some questions. Journal of Adolescent \& Adult Literacy, 53(5), 356-361. https://doi.org/10.1598/JAAL.53.5.1

Wicht, A., Rammstedt, B. \& Lechner, C. M. (2020). Predictors of Literacy Development in Adulthood. Insights from a Large-scale, Two-wave Study. Scientific Studies of Reading, 42, 1-9. doi.org/10.1080/10888438.2020.1751635

Wigfield, A., Gladstone, J., \& Turci, L. (2016). Beyond cognition: Reading motivation and reading comprehension. Child development perspectives, 10(3), 190-195. https://doi.org/10.1111/cdep.12184 
Wirthwein, L., Sparfeldt, J. R., Pinquart, M., Wegerer, J., \& Steinmayr, R. (2013). Achievement goals and academic achievement: A closer look at moderating factors. Educational Research Review, 10, 66-89. https://doi.org/10.1016/j.edurev.2013.07.001

Zinn, S., \& Gnambs, T. (2018). Modeling competence development in the presence of selection bias. Behavior research methods, 50(6), 1-16. https://doi.org/10.3758/s13428-018-1021-z 
GENDER DIFFERNECES IN READING COMPETENCE FROM ADOLESCENCE TO YOUNG ADULTHOOD

Table 1

Description of the measurement of reading competence

$\mathrm{T} 2$

T3

T4

summer 2011 winter 2013/2014 fall 2016/spring 2017

Grade 9

Grade 12 in school individual testing in all students in school setting testing in groups household individual testing in household

33 tasks

29 tasks easy booklet ( 25 tasks) easy and difficult booklet difficult booklet (27 tasks)

paper-and-pencil

paper-and-pencil

computer-based

assessments

assessments

assessment

13,897 valid WLEs

5,801 valid WLEs

6,863 valid WLEs

605 missing values
80 not participated ( $13 \%)$

8,701 missings values

7,639 missings values

2,673 not participated

(31\%)

6,904 not participated

525 not ascertainable

35 not ascertainable

$(90 \%)$

$(87 \%)$

(0.4\%)

17 not ascertainable

$(0.2 \%)$

5,993 missing by design

718 missing by design

(69\%)

$(10 \%)$
$\left(M_{W L E}=-0.02(S D=1.26)\right.$
$M_{W L E}=0.35(S D=1.00)$
$M_{W L E}=0.43(S D=0.96)$
IRT-based reliability
IRT-based reliability
IRT-based reliability
$(\mathrm{EAP} / \mathrm{PV})=.81$
$(\mathrm{EAP} / \mathrm{PV})=.80$
$(\mathrm{EAP} / \mathrm{PV})=.77$ 


\section{GENDER DIFFERNECES IN READING COMPETENCE FROM ADOLESCENCE TO YOUNG ADULTHOOD}

\section{Table 2}

Description of model variables

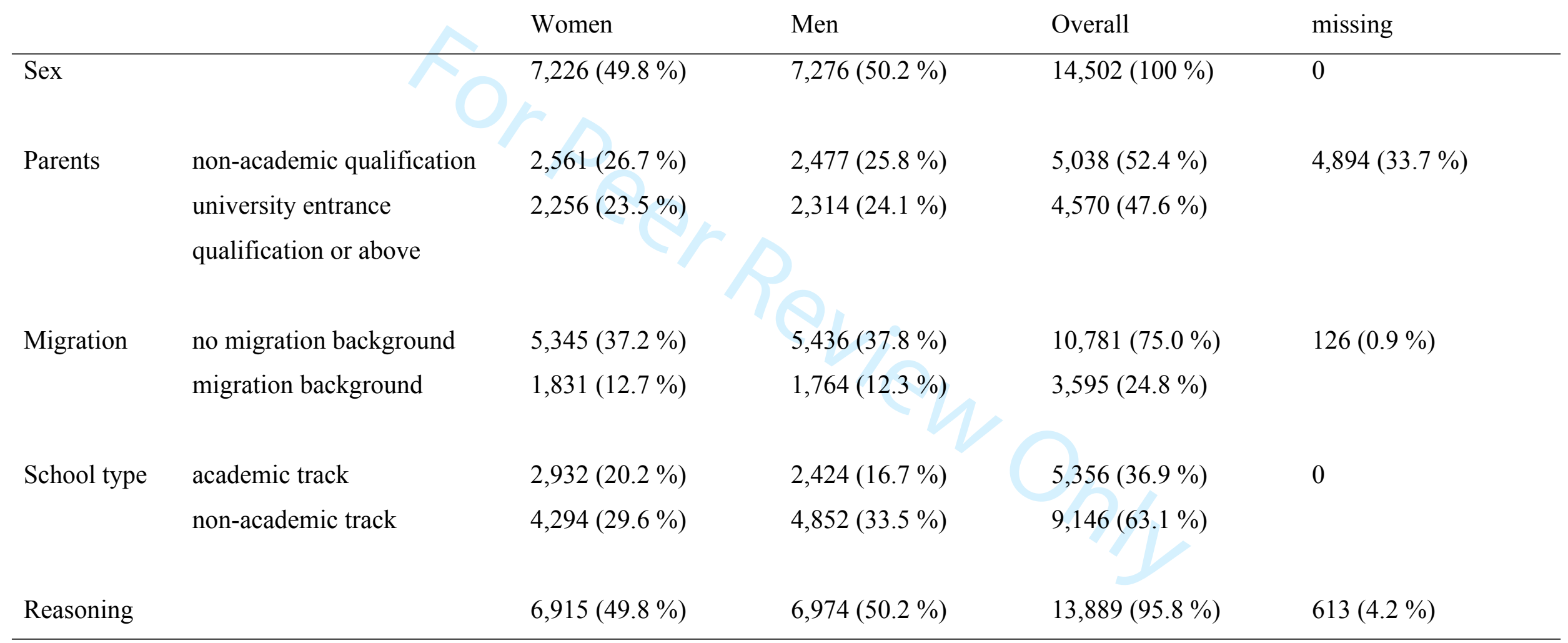


GENDER DIFFERNECES IN READING COMPETENCE FROM ADOLESCENCE TO YOUNG ADULTHOOD

Table 3

Manifest correlations between all variables of the final model for young women and young men

\begin{tabular}{|c|c|c|c|c|c|c|c|c|c|c|c|c|c|}
\hline & \multirow[b]{2}{*}{ Variables } & \multicolumn{2}{|c|}{1.} & \multicolumn{2}{|l|}{2.} & \multicolumn{2}{|c|}{3.} & \multicolumn{2}{|c|}{4.} & \multicolumn{2}{|c|}{5.} & \multicolumn{2}{|l|}{6.} \\
\hline & & Women & Men & Women & Men & Women & Men & Women & Men & Women & Men & Women & Men \\
\hline 1. & Reading T2 & - & & & & & & & & & & & \\
\hline 2. & Reading T3 & $.66^{*}$ & $.62 *$ & & & & & & & & & & \\
\hline 3. & Reading T4 & $.61 *$ & $.58 *$ & $.66^{*}$ & $.66^{*}$ & - & - & & & & & & \\
\hline 4. & Parents & $.26^{*}$ & $.23 *$ & $.27 *$ & $.25^{*}$ & $.28 *$ & $.26^{*}$ & - & - & & & & \\
\hline 5. & Migration & $-.22 *$ & $-.19 *$ &.$-.21 *$ & $-.17 *$ & $-.19 *$ & $-.18 *$ & -.02 & -.02 & - & - & & \\
\hline 6. & School type & $-.50 *$ & $-.46^{*}$ & $-.53 *$ & $-.45 *$ & $-.46^{*}$ & $-.42 *$ & $-.34 *$ & $-.32 *$ & $.12^{*}$ & $.11 *$ & - & - \\
\hline 7. & Reasoning & $.48 *$ & $.46^{*}$ & $.46^{*}$ & $.43 *$ & $.45^{*}$ & $.40 *$ & $.19 *$ & $.17^{*}$ & $-.18 *$ & $-.11 *$ & $-.39 *$ & $-.35 *$ \\
\hline
\end{tabular}

Notes. Reading longitudinal WLE (uncorrected for the position of the reading test within the booklet); Gender coding: female $=0$, male $=1$; parents' highest level of education: non-academic qualification $=0$, university entrance qualification or above $=1$; coding for migration background: no migration background $=0$, migration background $=1$; coding for school type: academic track $=0$, non-academic track $=1$.

$* p<.05$ 


\section{GENDER DIFFERNECES IN READING COMPETENCE FROM ADOLESCENCE TO YOUNG ADULTHOOD}

\section{Table 4}

Model results change and difference in reading competence

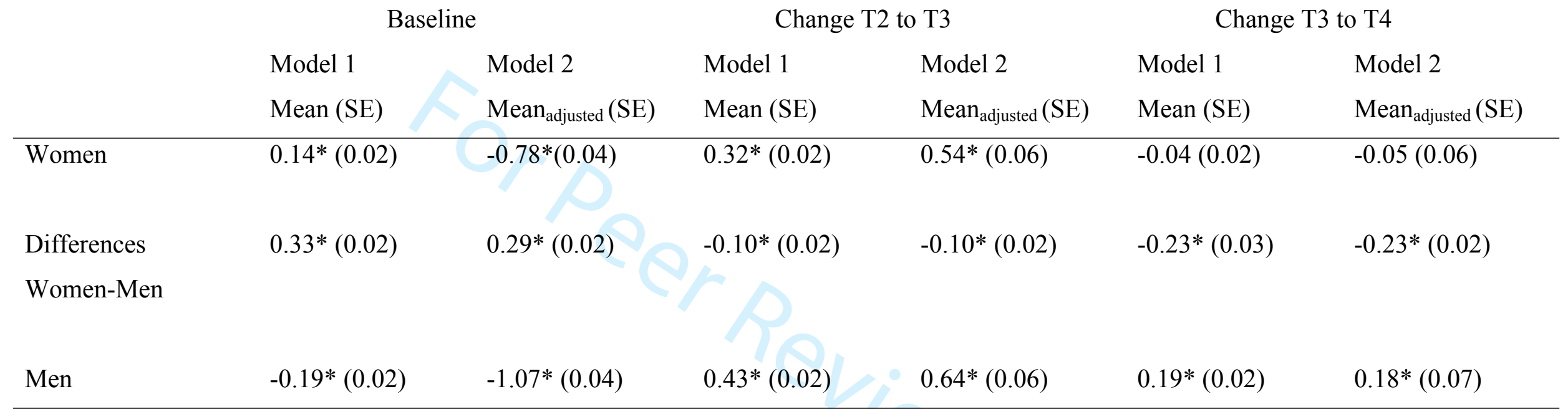

Notes. Reading longitudinal WLE (uncorrected for the position of the reading test within the booklet) $0=$ female; 1 = male

Model 1: means and standard estimates without controlling

Model 2: adjusted means and standard estimates for basic cognitive skills, parents' highest level of education, migration background, and school type on baseline and changes full information maximum likelihood (FIML) for missing values

$* \mathrm{p}<.001$ 
GENDER DIFFERNECES IN READING COMPETENCE FROM ADOLESCENCE TO YOUNG ADULTHOOD

two - group structural equation model separating young woman and young men

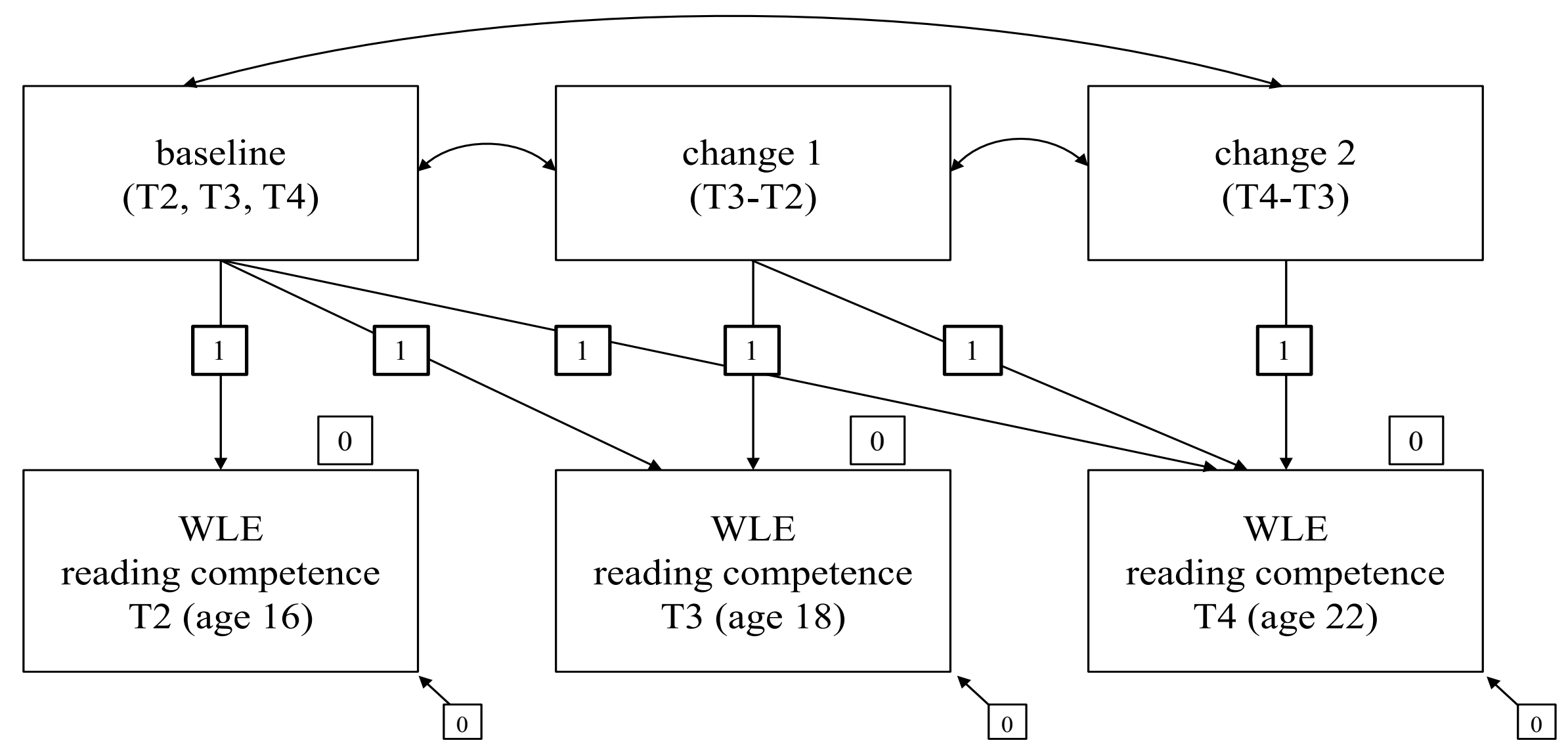

Figure 1. Neighbor change model for gender differences in reading competence

Notes. Reading competence longitudinal WLE (weighted maximum likelihood estimate; uncorrected for the position of the reading test within the booklet) 


\section{GENDER DIFFERNECES IN READING COMPETENCE FROM ADOLESCENCE TO YOUNG ADULTHOOD}

1,0

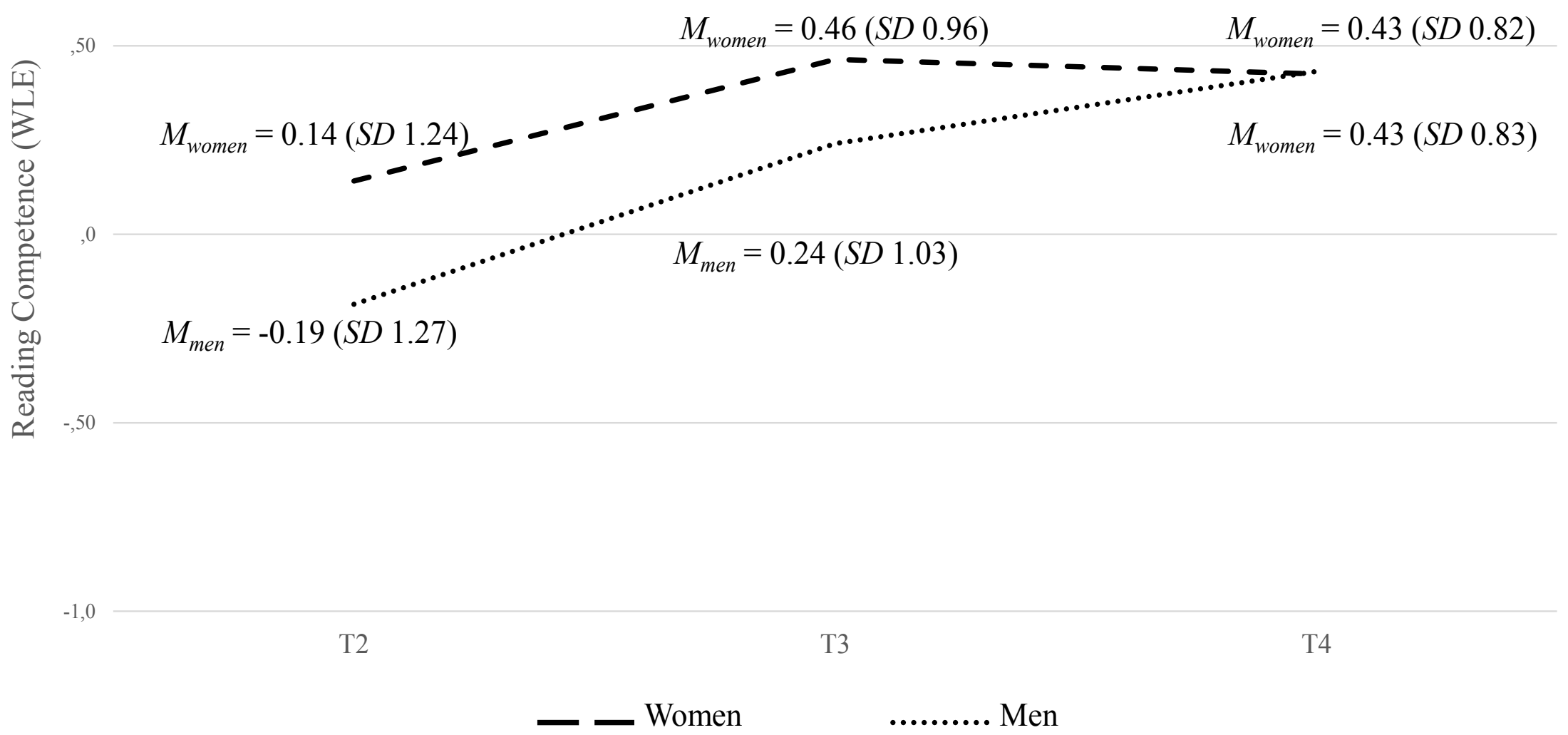

Figure 2. Reading competence development for women and men. Results of model 1, means and standard deviations without controlling for the covariates

Notes. T2 (age 16); T3 (age 18); T4 (age 22) 
Beitrag 2

Thums, K., Artelt, C., \& Wolter, I. (2020). Reading for entertainment or information reception? Gender differences in reading preferences and their impact on text-type-specific reading competences in adult readers. European Journal of Psychology of Education. Advance online publication. doi:10.1007/s10212-020-00486-1 


\title{
Reading for entertainment or information reception? Gender differences in reading preferences and their impact on text-type-specific reading competences in adult readers
}

\author{
Kathrin Thums ${ }^{1}$ (D) Cordula Artelt $^{1} \cdot$ Ilka Wolter $^{1}$ \\ Received: 20 September 2019 / Revised: 8 April 2020 / Accepted: 14 May 2020 \\ Published online: 06 June 2020 \\ (C) The Author(s) 2020
}

\begin{abstract}
This study aims at exploring gender differences in text-type-specific reading competences via readers' gender-specific reading preferences. Women were expected to read more often for the sake of entertainment (entertainment preference), whereas men were expected to read more often to gain information (information preference). We further assumed that individuals who read for entertainment would have higher reading competence in fictional literary texts compared with non-fictional informational texts, and vice versa for individuals who read to gain information. The analysis was based on a sample of 830 adults between 19 and 71 years $(M=31.04 ; S D=12.53)$ from a pilot study of the German National Educational Panel Study $(N E P S)$. A structural equation model confirmed that women and men show different reading preferences during leisure time. The preference to read for entertainment was predictive for reading competence in literary texts; however, the preference to read for information had positive effects on reading competence in both informational and literary texts.
\end{abstract}

Keywords Reading competence - Text-type-specific reading competences · Gender differences · Preferences to read $\cdot$ Reading behavior

Reading comprehension is a basic key competence for adults as well as for children and adolescents and is assumed to be indispensable for an active participation in various fields, including social life,

Kathrin Thums

kathrin.thums@lifbi.de

Cordula Artelt

cordula.artelt@lifbi.de

Ilka Wolter

ilka.wolter@lifbi.de

1 Leibniz Institute for Educational Trajectories, Wilhelmsplatz 3, 96047 Bamberg, Germany 
education and the labor market. Furthermore, reading is a key to learning and achievements in other domains since knowledge is largely accessible through text and discourse (Artelt et al. 2007; Organisation for Economic Co-operation and Development, OECD 2013, 2019). In this article, reading competence is conceptualized as reading literacy as defined in international large-scale assessments (LSA; e.g., PISA or PIRLS; cf. OECD 1999).

These LSA studies have revealed gender differences in reading competence between children and adolescents, whereas gender differences are small to non-existent in adulthood. Furthermore, a consistent pattern of gender differences in reading for children and adolescents, but not for adults, has become evident: In general, female students consistently prove to be more competent in reading than male students (Chiu and McBride-Chang 2006; Klieme et al. 2010; OECD 2019; Reiss et al. 2016).

Previous research suggests that specific features of the assessments are relevant for gender differences in reading competences. For example, the PISA study (OECD Programme for International Student Assessment) follows a design which is more in favor of girls due to the selection of continuous and literary texts (Solheim and Lundetræ 2017; Stanat and Kunter 2002). However, the reading assessment in the PIAAC study (Programme for the International Assessment of Adult Competencies), which is addressed to adult readers, consists of non-fiction texts and does not include narrative literary texts (OECD 2013; Solheim and Lundetræ 2017). Non-fiction texts are considered to be in favor of men over women, which might explain the small to non-existent gender differences in adults' competences in PIAAC (Solheim and Lundetræ 2017). Therefore, the focus of this study is not the general reading competence of women and men, but rather expands previous research on adults' reading competence by investigating gender differences in text types such as informational and literary texts (cf. Artelt and Schlagmüller 2004; Roick et al. 2013; Stanat and Kunter 2002).

However, a proficient reader is not only skilled in understanding texts and extracting relevant information, but also has a positive attitude towards reading (e.g., Artelt et al. 2010; Groeben 2004). This wider view of the concept of competence, including reading attitudes and motivational disposition, is also implemented in other frameworks and conceptualizations (e.g., Weinert 2001) and underlines the interdependence of motivation and achievement. Thus, the connection between the cognitive aspect of reading (reading competence) and the behavioral component based on reading preference might be a possible explanation for gender differences in reading competence. We expect that women and men have different reading preferences during leisure time. In particular, we argue that individuals' reading preferences during leisure time should predict gender differences in text-type-specific reading competence. Still, previous studies have not yet investigated whether gender differences in text-type-specific reading competence among adults are explained by gender differences in preferences of reading.

In summary, the present study expands previous research on gender differences in adults' reading competence by investigating reading competences with respect to text-type-specific reading competences. Moreover, the study aims at investigating if gender differences in texttype specific reading competence are mediated by adults' individual reading behavior (i.e., reading preferences during leisure time).

\section{Gender differences in reading competence}

Previous empirical studies on reading competence in different age groups throughout school consistently showed that girls were on average more proficient than boys (e.g., in primary school: Mullis et al. 2012; in secondary school: Klieme et al. 2010; OECD 2019; Reiss et al. 2016).

So far, in all PISA cycles, 15-year-old girls achieved significantly higher reading competence scores than their male peers (OECD 2019). In 2018, girls across all OECD countries 
achieved better reading competence scores than boys; in particular, they performed on average 30 points better than boys in the reading literacy assessment (girls: $M=502$ points, $S D=0.5$; boys: $M=472$ points, $S D=0.6$; OECD 2019). There are only a few studies on reading competences of adults and these reveal less clear results on gender differences in their reading abilities (OECD 2013; Solheim and Lundetræ 2017). The PIAAC study showed that there were no substantial gender differences, on average in the OECD countries, for adults between 16 and 65 years (OECD 2013). However, in PIAAC, the reading assessment is without including reading of narrative literary texts (OECD 2013; Solheim and Lundetræ 2017).

From an empirical point of view, it seems useful to differentiate between text types, e.g., informative texts as a form of expository non-fiction texts, and literary texts as a form of narrative fiction texts (Artelt and Schlagmüller 2004; Mar and Rain 2015; Roick et al. 2013). In order to follow the argument that reading preferences impact reading competence, the structural differences between different text types need to be identified. The textual requirements for reading and understanding expository non-fiction texts and narrative fiction texts also differ with regard to their language and their formal structure and in terms of content (Gardner 2004). Research indicates that literary text competence and informative text competence are distinguishable text-type-specific reading competences and a differentiation of specific text types allows a more precise view on competence differences.

Following this argument, Stanat and Kunter (2002) further analyzed the data from the international PISA sample regarding gender-specific strengths and weaknesses in reading competence scores dependent on text formats (continuous texts and non-continuous texts). The 15-year-old girls performed 0.5 standard deviation better than boys in continuous texts (e.g., continuously written texts such as narrations and arguments); however, they only outperformed boys by 0.1 standard deviation in non-continuous texts (non-continuous information, like charts, diagrams, and graphics). When children's interest in reading was controlled for, there was even a slight advantage for boys in non-continuous texts. Yet, girls' better performance in continuous texts remained significant even when controlling for interest. Therefore, gender differences in reading can also be ascribed to the type and presentation of texts (Stanat and Kunter 2002).

Artelt and Schlagmüller (2004) also differentiated PISA texts with respect to text types. The authors argued that the competent handling of literary texts is a specific aspect of reading competence, because with literary texts, different rhetorical requirements like metaphors or symbols are used. They further categorized continuous texts dependent on their content either to literary texts (narrations, e.g., fable, drama, or parts of novels) or to other continuous texts (e.g., statement, description) and distinguished them from non-continuous texts including charts, tables, and diagrams. The authors hypothesized that girls' better performance in reading is not only due to the text format, but also to the content of texts. It was presumed that reading literary texts is a typical activity for girls, and therefore they should achieve substantial performance advantages on literary texts as compared to boys. Consequently, the advantage for girls in other continuous texts was presumed to be much smaller than with literary texts. However, the results of this study showed that girls were more competent than boys in reading continuous text types, irrespective of the distinction into literary texts and other continuous texts. Additional analysis showed that gender differences in favor of girls were less pronounced when processing non-continuous texts than continuous ones.

To summarize previous empirical results, there were gender differences in reading competence for girls and boys of school age, whereas there were no gender differences in adults' reading competence, though it is important to note that reading abilities, as measured in studies 
with adults cohorts like in PIAAC (16-65 years), do not refer to the same framework of reading comprehension as measured in school-based large-scale assessments such as PISA (cf. Lundetræ et al. 2014), for example narrative texts are not included in PIAAC as compared to PISA (Solheim and Lundetræ 2017). Moreover, less research has been conducted on gender differences in reading competence of adults and, furthermore, those few studies lack a more detailed focus on text types, e.g., narrative texts. Although an increasing number of studies consider the general reading competence of adults, little is known so far about text-typespecific reading competence of adults. As mentioned by Artelt and Schlagmüller (2004) and Roick et al. (2013) as well as Stanat and Kunter (2002), the distinction of specific text types might be relevant when exploring gender differences in reading competence. To our knowledge, the differentiation of text types, however, has never been examined in adults' reading competence. Therefore, this study aims at investigating gender-differentiated reading competence of adults in literary and informational texts.

\section{The impact of reading behavior on reading competence}

Reading and understanding texts play an important role in many areas of everyday life; therefore, the impact of reading behavior on reading competence should be looked at more closely. Previous research showed a positive correlation between reading behavior (or habits, intentions, preferences) and different reading skills (e.g., word reading and recognition, reading comprehension) (Mar and Rain 2015; McGeown et al. 2015; Mol and Bus 2011; Torppa et al. 2019). Pfost et al. (2013) called this reciprocity a "virtuous circle of reading." While the reader makes an authentic reading experience, the development of reading competence is fostered and their reading volume increased (Pfost et al. 2013).

In a meta-analysis with 99 studies $(N=7669)$, Mol and Bus (2011) examined three age groups (kindergarten/pre-school, first-12th grade, and university students). In all three age groups, print exposure correlated ( $r=0.30$ to $r=0.65$ ) with different components of reading competence (oral language, reading comprehension, basic reading skills, word recognition, and spelling). With increasing age, the impact of reading volume became more relevant for the comprehension components, especially for oral language and word recognition. Noteworthy is the close link between reading practice and the components of reading competence among students. The authors argued that this high relationship reflects the independent choice of reading and the self-determined selection of leisure activities.

Pfost et al. (2013) also found in a sample with 1226 children (age $M=11.4$ years; $S D=$ 0.46 ) that reading narrative texts or books (novels, narratives, and stories) in their leisure time was the best predictor for the development of reading comprehension and vocabulary. The quantity of newspapers, comics, and non-fiction books was, however, less important for reading competence development. The authors reveled that different texts have different characteristics and thus define different requirements for the readers and their reading comprehension.

Other studies also investigated the role of reading behavior in leisure time for the development of reading literacy, especially of vocabulary, reading speed, and reading comprehension. In a sample of 312 students between 11 and 16 years, McGeown et al. (2015) found that fiction reading was a significant predictor of reading comprehension, summarization skills, and reading speed. In this study, girls reported more fiction book reading than boys. 
Mar and Rain (2015) examined university students at the age of 20 years who were asked to report on their reading habits and preferences, and participate in a recognition test for print exposure. Findings revealed that exposure to narrative fiction was a better predictor for verbal abilities (synonym knowledge, analogies, sentence completion, and reading comprehension) than expository non-fiction.

To sum up, previous research showed a positive impact of leisure time reading behavior on reading competence and we assume that the quantity of reading in leisure time has a positive influence on reading competences (Mol and Bus 2011).

\section{Reading behavior as a readers' characteristics for a positive impact of reading competence}

It should, however, also be noted that reading behavior is very diverse and multifaceted (Christmann 2004; Groeben 2004). In particular, in the area of leisure time reading, when readers can freely choose their reading material as compared to a professional context, a large number of possible genres, topics, and media formats (paper versions or digital formats) are available to readers who can choose freely according to their own interests, habits, and intentions. In our study, we focus on reading preferences in leisure time since it is plausible to assume that the amount of reading and the specific reading material during leisure time reading is more autonomously and voluntary selected than when reading in the work context. We hypothesized that during leisure time, readers select their reading material independently according to their own preferences from a wide range of existing reading material and genres.

Next to focusing solely on characteristics of text features, it is important to also acknowledge the characteristics of readers, for example their reading preferences and prior knowledge, as well as the interaction of text features and readers' characteristics for reading competence (Artelt et al. 2001; Christmann 2004). Consequently, there are fluent transitions between the genres and topics of reading material, as well as the readers' reading behavior, habits and preferences. Thereby, it is plausible to assume that readers pursue multiple intentions with their choices and preferences for reading materials or topics. Groeben (2004) argued in his theoretical model that readers most frequently choose certain text genres based on different reading preferences. Different text genres serve more or less diverse purposes for the reader, e.g., to provide entertainment or to gain information. Hence, the author distinguished two opposite functions of reading texts: entertainment texts (e.g., literary, fictional) and informational texts (e.g., pragmatic, non-fiction). This distinction was described as two separate extremes, but should rather be understood as a continuum since there are also multiple mixed forms of text functions and reader preferences (Groeben 2004).

Understanding and perception of texts as well as fostering esthetic sensitivity and linguistic differentiation are common functions of both entertainment and informational texts. In the narrower sense, this presupposes a corresponding reading competence of the reader. A further common function of reading preferences is enabling the reader to reflect on possible and real worlds (Groeben 2004).

The priority functions of fictional texts (e.g., novels or stories) are entertainment functions at the process level, such as building tension, eliciting joy and sorrow, providing esthetic pleasure, and withdrawing from everyday life (Groeben 2004). 
The predominant functions of informational texts (e.g., non-fiction texts, newspapers, magazines) are aspects of knowledge acquisition, assessment, justification and attitude formation, and evaluation (Groeben 2004).

By reading texts, social and individual consequences are intended for the readers. Entertainment texts mainly foster imagination and strengthen moral awareness. Furthermore, those texts allow the reader to familiarize themselves with topics of diversity and increase their ability to feel empathy. Informational texts essentially foster argumentative communication and (political) opinion formation as well as gaining knowledge (Groeben 2004).

Also, there is an overlap of entertainment and informational functions and genres. Therefore, a categorical separation is difficult in some cases. The distinction in two separate scales is not a distinction in one or the other, rather a value indicating how both functions work relative to each other. The values specify what a reader most often selects from the wealth of possible genres. A reader may have the preference to read both of them equally. Against this background, we assume a positive effect of adults' reading preferences on their reading competence in literary and informational texts.

\section{Gender differences in reading behavior}

A frequently used explanation for gender differences in reading competence is a genderspecific development in terms of reading motivation and interests for reading. Thus, the competence differences have often been attributed to differences in reading interest and enjoyment or intrinsic reading motivation (e.g., Chiu and McBride-Chang 2006; Retelsdorf et al. 2011; Schiefele et al. 2012).

In 2008, the German Foundation for Reading examined 2552 individuals, aged 14 and older. Participants were asked to indicate the frequency of reading periodicals and books. Almost twice as many women as men (37\% vs 20\%) read books for entertainment, whereas men were more likely drawn to realistic facts than women ( $51 \%$ vs $42 \%)$. Compared to men, women more often wanted to forget everyday life (52\% vs $33 \%$ ), to be empathic with other figures (31\% vs 19\%), or to escape into another world (28\% vs 17\%) (Stiftung Lesen 2008). These descriptive results coincide with Groeben's (2004) theoretical considerations on the textual functions of entertainment and informational literature.

Furthermore, Charlton et al. (2004) conducted an interview study with 1025 individuals between 16 and 88 years $(M=42$ years) on their emotional engagement with literary texts. They found significant gender differences in the extent to which women and men identify and empathize with the texts and use them to learn about life. The results suggested that women applied different emotional strategies to deal with literary texts. Women seemed to identify more with the characters, to sympathize with the characters, and to use the topics for their own everyday life. In a further analysis, Burbaum et al. (2004) also found that men read more for work than women, and that more women than men used reading to cope with life.

Schreier and Odağ (2004) confirmed in a data re-analysis that women were more involved with fictional texts, whereas men were consistently better at drawing the line between fiction and their everyday lives.

In summary, there is empirical evidence for distinct preferences to read entertainment texts and informational texts (Groeben 2004). Moreover, there are in general gender differences in reading preferences. It seems that women were more involved in the reading material and got further in touch with the content and more often read fiction books for their entertainment. 
Men, however, more strongly differentiated between reality and fiction and were less emotionally involved with what they were reading. Thus, men more often wanted to gain knowledge and facts through the reading material than women (Burbaum et al. 2004; Charlton et al. 2004; Schreier and Odağ 2004).

\section{Research aims and hypotheses}

Against this background, the present study aims to contribute to research on gender differences in adults' reading competence by (a) investigating gender differences in text-type-specific reading competences and (b) taking the varying reading preferences of women and men into account.

Since there is on average no gender difference in general reading competence of adults (OECD 2013), we focus on competences in specific text types (i.e., narrative literary fiction and informational non-fiction texts), because this has not been studied more closely for adults. Previous studies showed that in adolescence, there are gender difference in reading competence and especially in text-type-specific reading competence (Artelt and Schlagmüller 2004; Stanat and Kunter 2002). Therefore, we also expected that if it is possible to distinguish general reading competence into text-type-specific reading competence, different results for women and men become apparent in reading competence of informational and literary texts.

Hence, we expected that reading in leisure time would have a positive impact on reading competence in both text types. However, since there is also empirical evidence for different reading preferences of women and men in their leisure time (Burbaum et al. 2004; Charlton et al. 2004; Schreier and Odağ 2004), we suggest that gender differences in reading competence of literary and informational texts may be mediated by gender-specific reading preferences.

The hypotheses are as follows:

1. There are gender differences in reading competence of literary and informational texts. We expect that women have a higher text-type-specific reading competence in literary texts and men have higher text-type-specific reading competence in informational texts.

2. Reading preferences are related to individuals' reading competences, dependent on text types: Individuals who read for entertainment have higher reading competence in literary texts compared to informational texts.

Individuals who read to gain knowledge have higher reading competence in informational texts than in literary texts.

3. There are gender differences in reading preferences: Women prefer to read to entertain themselves, whereas men are more likely to read for the purpose of knowledge acquisition.

\section{Methods}

\section{Sample and design}

The data was collected within a pilot study of the German National Educational Panel Study (NEPS; Blossfeld et al. 2011). The sample was selected through a predefined quota scheme of 
university students and adults. The categories for the stratified random sampling were age, and education.

Of the 939 study participants in the domain reading, only 900 participants were considered because of an experimental design in which a small subsample was assessed in a different test condition. Furthermore, due to the low number of participants with a migration background $(n=70)$, only those with German as a first language and without a migration background were included in the analyses. This resulted in a final sample of $n=830$ adults between 19 and 71 years $(M=31.04 ; S D=12.53$; exact distribution see Table 1$)$. About half of the participants were female $(n=431 ; 51.9 \%)$. The majority of the sample had an upper secondary school leaving certificate as the highest level of school education in Germany (i.e., Abitur, university entrance degree; exact distribution see Table 1). An exact distribution of the participants by gender, age, and education is available in an open science document: https://osf.io/ma7n5/.

\section{Research instruments}

The study was conducted in the participants' own household with a computer-based assessment of their reading competence and a subsequent questionnaire about their sociodemographic background variables and reading preferences. A trained interviewer was present at the assessment and proctored the testing situation throughout the competence test and questionnaire.

Reading competence assessment A total of 20 texts were used in the pilot study to investigate the reading competence in German. According to the theoretical framework of NEPS, the reading texts cover five different text types: informational texts, commenting or argumentative texts, literary texts, instructional texts, and advertising texts (cf. NEPS framework for assessing reading competence; Gehrer et al. 2013). For the present study, only participants' responses on tasks related to literary and informational texts were analyzed. Therefore, the final material used in the analyses consisted task responses to six informational texts and three literary texts. Task formats were dichotomous multiple choice items and polytomous complex multiple choice items (e.g., ordering items, and cloze items).

In the nine texts, a total of 67 tasks (46 tasks in informational texts, 21 tasks in literary texts) were presented to the participants. The tasks were scaled based on an IRT framework with a

Table 1 Sample distribution in age and highest level of school education

\begin{tabular}{lll}
\hline & $N$ & Percent \\
\hline Age & & \\
19-25 years & 478 & 57.6 \\
26-45 years & 219 & 26.4 \\
46-71 years & 133 & 16.0 \\
Highest level of school education & 2 & 0.2 \\
Did not complete any form of schooling & 5 & 0.6 \\
Special school leaving certificate & 61 & 7.3 \\
Basic Hauptschule leaving certificate & 36 & 4.3 \\
Qualifying Hauptschule leaving certificate & 137 & 16.5 \\
Realschule leaving certificate & 55 & 6.6 \\
University of applied sciences entrance qualification (Fachhochschulreife) & 534 & 64.3 \\
University entrance qualification (Abitur) & & \\
\hline
\end{tabular}


two-parametric logistic model (2PL; Birnbaum 1968) using the ConQuest software package (version 4.5.2; Adams et al. 2015). The reading competence of individuals was provided by a weighted maximum likelihood estimate (WLE) on a logit scale with a mean standardized to zero. Two WLEs for the reading competence were estimated, each for literary texts and informational texts. The prior selection of the tasks was conducted with regard to the item quality (i.e., WMSQ, ICC; cf. Rost 2004) and the discrimination parameters of the items. Moreover, a differential item functioning analysis for testing measurement invariance across women and men was implemented (cf. Pohl and Carstensen 2013). Twelve tasks did not meet the quality criteria (WMSQ $>1.1$; low correlations $<0.20$; DIF between women and men) and were excluded due to their low item quality. As a result, 55 tasks were finally included in the estimation of the WLEs (informational texts, 37 tasks; marginal reliability, 0.70; literary texts, 17 tasks; marginal reliability, 0.60).

Reading preferences in leisure time The preferences of reading material were measured by a questionnaire and operationalized by the prompted reading behavior during leisure time. The participants were asked to indicate from a list of genres how often they usually read these in their leisure time: crime fiction, newspaper, factual books, biography, science fiction, novels, and thriller or horror books on a 5 -point scale $(1=$ never/seldom; $2=$ several times a month; $3=$ once a week; $4=$ several times a week; $5=$ daily). It should be underlined that the questions regarding reading genres were not specified to certain media formats. Since reading preferences in this study were aimed at genres, which can be read in any medial form, we presume that print and digital media are included in this question.

On the basis of the theoretical remarks of Groeben (2004), the genres of science fiction, novels, crime fiction, and thrillers or horror books were categorized into a formative index of the preference to read for entertainment. These genres represent the preference of reading fictional entertainment literature under emotional-motivational aspects as a self-purpose and for entertainment.

The genres of newspaper, factual books, and biography were categorized into the formative index of the preference to read non-fiction informational literature. According to Groeben (2004), they indicate the preference of reading non-fiction in order to obtain information and generate knowledge.

The formative approach of building the indicators was used because the theoretical concept from indicators of preference to read formed the dependent variable of reading competence (Curtis and Jackson 1962; Weiber and Mühlhaus 2014). The two indicators of preference to read form two independent scales. Consequently, readers might have high or low values on both scales or show a mixed pattern of reading preferences.

Choices of reliability tests of a formative model are severely limited. The reliability test in a reflexive model (e.g., Cronbach's alpha) aims for a high correlation between the tasks of an indicator. The indicators of a formative model, however, should be broadly independent from each other. The variance inflation factor (VIF) is therefore used to control the quality of indicators in a formative model (Weiber and Mühlhaus 2014). Diamantopoulos and Riefler (2008) suggested a cutoff value for the VIF from $>5$.

In the analyses for this study, the correlations of the indicator of preference to read for entertainment were between $r=0.12$ and $r=0.53$ (thriller or horror books and crime fiction). The VIF values were between 1.04 and 1.45. The correlations of the indicator of preference to read for information were between $r=0.13$ and $r=0.18$. The VIF values were between 1.02 and 1.03 . 
Both the correlation matrix and the VIF values did not point to the presence of multicollinearity. Therefore, formative indicators of the preference to read for entertainment and the preference to read for information were provided.

Control variables Various studies have shown that reading competence is associated with the number of books at home (e.g., Chiu and McBride-Chang 2006); therefore, we decided to use the number of books at home as control variable. The number of books at home was measured on a 6-point scale (from 0 to 10 books to more than 500 books).

Furthermore, due to the wide span of age in the sample (19-71 years), participants' age was also included as a continuous control variable in the model.

\section{Analysis strategy}

A mediation model was computed with reading competence in literary and informational texts as dependent variables, preferences to read for entertainment or information as mediator variables, and participants' gender as predictor variable. Gender was included as effect coded variable $(-0.5=$ female; $0.5=$ male $)$. The number of books at home and age were grand-mean centered and entered as control variables in the model.

The analysis of the mediation model was conducted using the software Mplus (edition 7; Muthén and Muthén 2017). The Mplus syntax with the model output is available under https://osf.io/ma7n5/.

To test the significance of indirect effects in the model, the product of two direct paths needs to be interpreted. However, the products of direct paths are usually not normally distributed; therefore, conventional significance tests (e.g., joint significance test, Baron and Kenny 1986) have only limited reliability. To address this problem in the mediation model, a standard non-parametric bootstrapping method was used to estimate the four indirect effects and 1000 bootstrap samples were drawn (cf. MacKinnon 2013).

Furthermore, to handle the missing values in the mediator variables, a full information maximum likelihood (FIML) estimator was used (Allison 2009; Enders and Bandalos 2001). The amount of missing data was overall very low and ranged between $2.5 \%$ (preferences to read for information) and $2.7 \%$ (preferences to read for entertainment), and $0.2 \%$ for the number of books at home.

\section{Results}

\section{Descriptive statistics}

Table 2 displays the means and standard deviations of the WLEs for reading competence in literary texts and informational texts, the preferences to read for entertainment and information, and the control variables' number of books at home and individual's age. The descriptive statistics are displayed for the whole sample and separated by gender. Furthermore, the $t$-values for the mean gender difference are included in Table 2. Since reading preferences were measured with formative indicators, sum values were provided for both variables. These two sum values cannot be compared with each other because a different number of tasks were included in the calculation of those values. Each reading preference should therefore be considered a separate scale regarding gender differences in text-type-specific reading competences. 
Table 2 Sample size, mean, and standard deviations for the dependent, mediator, and independent variables as well as mean differences for women and men

\begin{tabular}{lllllll}
\hline & $N$ & $M(S D)$ & $M_{\text {Female }}(S D)$ & $M_{\text {Male }}(S D)$ & $t(d f)$ & $d$ \\
\hline $\begin{array}{l}\text { Reading competence } \\
\text { in literary texts }\end{array}$ & 754 & $0.02(1.48)$ & $0.07(1.21)$ & $-0.02(1.23)$ & $0.99(752)$ & -0.07 \\
$\begin{array}{l}\text { Reading competence } \\
\quad \text { in informational texts }\end{array}$ & 829 & $0.02(1.23)$ & $-0.03(1.11)$ & $0.07(1.11)$ & $1.40(827)$ & 0.09 \\
$\begin{array}{l}\text { Preference to read } \\
\text { for entertainment }\end{array}$ & 808 & $6.22(2.53)$ & $6.64(2.52)$ & $5.79(2.48)$ & $4.83 *(806)$ & -0.34 \\
$\quad \begin{array}{l}\quad 4 \text { variables, sum score) } \\
\text { Preference to read }\end{array}$ & 809 & $6.50(2.32)$ & $6.30(2.31)$ & $6.75(2.31)$ & $2.77 *(807)$ & 0.20 \\
$\quad$ for information & & & & & & \\
$\quad(3$ variables, sum score) & & & & & & \\
$\begin{array}{l}\text { Number of books at home } \\
\text { Age }\end{array}$ & 828 & $3.62(1.43)$ & $3.68(1.40)$ & $3.55(1.46)$ & $1.23(826)$ & -0.09 \\
\hline
\end{tabular}

Gender coding: $-0.5=$ female; $0.5=$ male; coding for preference to read: $1=$ never/seldom, $2=$ several times a month, $3=$ once a week, $4=$ several times a week, $5=$ daily; coding for books: $1=0-10$ books, $2=11-25$ books, $3=26-100$ books, $4=101-200$ books, $5=201-500$ books, $6=$ more than 500 books

Reading competence scaled as a weighted maximum likelihood estimate (WLE) on a logit scale with an average mean of zero

$* p<0.05$

The descriptive analyses showed no gender differences in reading competence in literary and informational texts. Furthermore, there were significant gender differences in reading preferences. As expected, women read more often for entertainment (science fiction, novels, crime fiction, and thrillers or horror books) than men. However, men have a significantly higher reading preference for informational texts (newspaper, factual books, and biography) than women. Moreover, there were no significant gender differences in the control variables (i.e., number of books at home and age).

A bivariate correlation matrix with all included variables of the final model is presented in Table 3. The correlations of the included variables range from $r=0.00$ to $r=0.64$ with relatively low coefficients. There was one exception; the correlation between reading competence in literary texts and informational texts $(r=0.64)$ was rather high; however, they still represent separate constructs. The correlation between the two separate scales of reading preferences for entertainment and information was very low $(r=0.07)$. The correlations between reading competence in informational texts and reading preferences were $r=.14$ for preference to read for entertainment and $r=.22$ for preference to read for information. The correlations between reading competence in literary texts and reading preferences were $r=.18$ for preference to read for entertainment and $r=.20$ for preference to read for information.

\section{Mediation model}

Analyses of direct paths The mediation model (see Table 4 and Fig. 1) showed a small gender difference in reading competence in informational texts after including reading preferences and control variables. In that case, men had slightly higher reading competences in informational texts than women $(b=0.15, S E=0.07, p=0.04, d=0.14)$, although there was no gender difference in the reading competence in literary texts $(b=-0.02, S E=0.08, p=$ 0.82 ). So the first hypothesis was partly confirmed, that there are small gender differences in 
Table 3 Correlations between all variables of the final model

\begin{tabular}{llllllll}
\hline & Variables & 1 & 2 & 3 & 4 & 5 & 6 \\
\hline 1 & Reading competence in literary texts & - & & & & \\
2 & Reading competence in informational texts & $0.63^{*}$ & - & & & & \\
3 & Preference to read for entertainment & $0.18^{*}$ & $0.14^{*}$ & - & & & \\
4 & Preference to read for information & $0.20^{*}$ & $0.22^{*}$ & $0.07^{*}$ & - & & \\
5 & Gender & -0.03 & 0.05 & $-0.17^{*}$ & $0.09^{*}$ & - \\
6 & Number of books at home & $0.33^{*}$ & $0.37^{*}$ & $0.32^{*}$ & $0.33^{*}$ & -0.04 & - \\
7 & Age & $-0.26^{*}$ & $-0.28^{*}$ & -0.00 & $0.09^{*}$ & 0.03 & $0.08^{*}$ \\
\hline
\end{tabular}

Pearson correlations, gender coding: $-0.5=$ female; $0.5=$ male; ${ }^{*} p<.05$

informational texts in favor of men, but there were no difference between women and men in reading competence regarding literary texts.

The second hypothesis is related to the research question whether reading competence in text-type-specific reading is predicted by preferences for reading. Individuals who read for entertainment had slightly higher reading competence in literary texts $(b=0.04, S E=0.02, p=$ $0.04, d=0.14$ ), whereas there was no effect from the preferences to read for entertainment on reading competence in informational texts $(b=0.01, S E=0.01, p=0.39)$.

Additionally, there were significant effects from the preference to read for information on the reading competence in literary texts $(b=0.07, S E=0.02, p<0.01, d=0.27)$ as well as on the reading competence in informational texts $(b=0.06, S E=0.02, p<0.01, d=0.27)$. Therefore, hypothesis 2 was partly confirmed because individuals who read for entertainment had a

Table 4 Mediation model: gender differences in text-type-specific reading competences as mediated by preferences to read for entertainment or information

\begin{tabular}{|c|c|c|c|c|}
\hline & \multirow[b]{2}{*}{$b$} & \multicolumn{2}{|c|}{ Model } & \multirow[b]{2}{*}{$d$} \\
\hline & & S.E. & $\beta$ & \\
\hline \multicolumn{5}{|l|}{ Reading competence in literary texts on } \\
\hline Preference to read for entertainment & $0.04 *$ & 0.02 & 0.08 & 0.14 \\
\hline Preference to read for information & $0.07 *$ & 0.02 & 0.13 & 0.27 \\
\hline Gender & -0.02 & 0.08 & -0.01 & -0.02 \\
\hline Number of books at home & $0.24 *$ & 0.03 & 0.28 & 0.58 \\
\hline Age & $-0.03 *$ & 0.00 & -0.29 & -0.67 \\
\hline \multicolumn{5}{|c|}{ Reading competence in informational texts on } \\
\hline Preference to read for entertainment & 0.01 & 0.01 & 0.03 & 0.06 \\
\hline Preference to read for information & $0.06^{*}$ & 0.02 & 0.13 & 0.27 \\
\hline Gender & $0.15^{*}$ & 0.07 & 0.07 & 0.14 \\
\hline Number of books at home & $0.27 *$ & 0.03 & 0.35 & 0.74 \\
\hline Age & $-0.03^{*}$ & 0.00 & -0.32 & -0.84 \\
\hline \multicolumn{5}{|l|}{ Preference to read for entertainment on } \\
\hline Gender & $-0.79 *$ & 0.17 & -0.16 & -0.34 \\
\hline Number of books at home & $0.57 *$ & 0.06 & 0.32 & 0.79 \\
\hline Age & -0.00 & 0.01 & -0.02 & -0.04 \\
\hline \multicolumn{5}{|l|}{ Preference to read for information on } \\
\hline Gender & $0.50 *$ & 0.16 & 0.11 & 0.23 \\
\hline Number of books at home & $0.54 *$ & 0.06 & 0.33 & 0.73 \\
\hline Age & 0.01 & 0.01 & 0.06 & 0.13 \\
\hline
\end{tabular}

Gender: $-0.5=$ female, $0.5=$ male; control variables: books and age grand mean centred; $d=$ effect size according Cohen's $d$.; * $p<0.05$ 


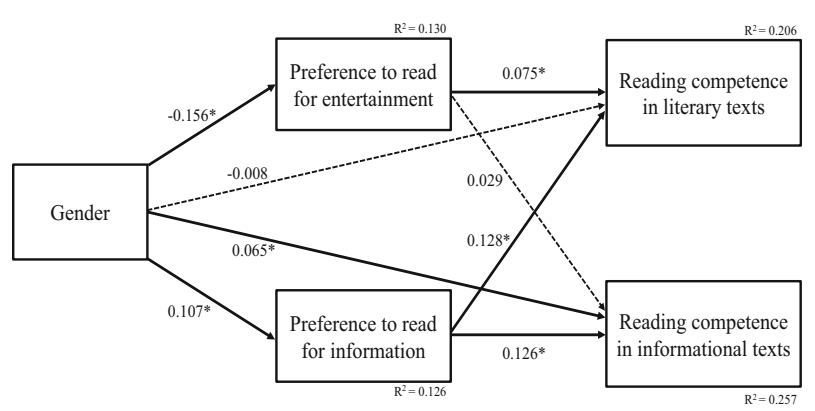

Fig. 1 Mediation model: gender differences in text-type-specific reading competences as mediated by preferences to read for entertainment or information; standardized regression parameters are displayed in the figure; dashed lines are not significant. $* p<0.05$

slightly higher reading competence in literary texts compared to informational texts. However, individuals who read to gain knowledge have higher reading competence in informational texts, yet, opposite to our hypothesis, they also showed higher reading competence in literary texts.

The third hypothesis could be confirmed that there were gender differences in the preferences to read. Women showed a higher preference to read for entertainment (science fiction, novels, crime fiction, and thrillers or horror books; $b=-0.79, S E=0.17, p<0.01, d=-0.34$ ) than men, whereas men preferred to read to gain information (newspaper, factual books, biography; $b=0.50, S E=0.16, p<0.01, d=0.23$ ).

Analyses of indirect paths The indirect paths from gender on text-type-specific reading competences, mediated by reading preferences, were also analyzed. The indirect effects from gender on reading competence in literary texts $(b=-0.03, S E=0.02, p=0.06)$ or on informational texts $(b=-0.01, S E=0.01, p=0.40)$, mediated by the preference to read for entertainment, were not significant. Furthermore, the indirect path from gender on the reading competence in informational texts, mediated by preference to read for information, was significant with a small effect size $(b=0.03, S E=0.01, p=0.01, d=0.17)$. The indirect effect from gender on reading competence in literary texts mediated by preferences to read for information was also significant with a similar effect size $(b=0.03, S E=0.01, p=0.02, d=$ $0.17)$.

\section{Discussion}

The main goal of the study was to explore gender differences in reading competences in literary and informational texts based on different reading preferences of women and men in their leisure time. The study extended previous research on gender differences in pre-school and school age children (e.g., OECD 2019; Stanat and Kunter 2002) by focusing on the reading competence of adults and investigating gender differences in text-type-specific reading competences. Furthermore, the present study focused on an alternative behavioral component, namely reading preference during leisure time, as an explanation of gender differences in the cognitive aspect of reading (i.e., reading competence). 
Interestingly, in this study, there were descriptively no gender differences in adults' reading competence in literary or informational texts. Even though there are only few studies with respect to adults' reading competence, this finding is in line with previous results on gender differences in reading abilities of adults (e.g., PIAAC; OECD 2013).

Furthermore and in line with our hypothesis, the preference to read for entertainment had a significant but small influence on the text-type-specific reading competence in literary texts, but no significant impact on reading competence in informational texts. Additionally, the preference to read for information had an impact on reading competences in both informational and literary texts. The latter was unexpected and is not in line with previous research. Pfost et al. (2013) as well as Mar and Rain (2015) showed for example for children and university students at the age of 20 that reading narrative texts was more important for the development of reading skills than reading expository texts. This inconsistency might be explained by specific characteristics of our sample which consisted of adults and university students with a broader age range (19-71 years) as compared to previous studies (Mar and Rain 2015; Pfost et al. 2013). Moreover, due to an oversampling of university students, our sample is also characterized by high educational attainment. The leisure time reading behavior of university students, as well as adults up to the age of 71, thus, might represent a different meaning than those of secondary school students as investigated by Pfost et al. (2013). Furthermore, the need and necessity of gaining information by reading might be more pronounced in adults than in secondary school students; hence, informational texts, for example newspapers, are in general more often used as reading material. In future research with higher number of participants in various age clusters, it would be interesting to focus on different age ranges in order to obtain more differentiated results on reading behavior and text-type-specific reading competence.

As expected, women read more often for entertainment (science fiction, novels, crime fiction, and thrillers or horror books) in their leisure time, whereas men more often preferred to read to gain information (newspaper, factual books, biographies). These results confirmed previous research which showed that women are in general more involved with the content of their reading material, and eventually more frequently choose fictitious reading material than men in their leisure time. Moreover, prior research revealed that men in general distinguish more strongly between reality and fiction and are not as involved with their reading contents (Burbaum et al. 2004; Charlton et al. 2004; Schreier and Odağ 2004). In the present study, gender differences in reading preferences were assessed by the frequencies of reading specific text genres. Thus, it was possible to ascribe reading preferences to concrete text functions in literary and informational texts.

The findings of the present study also showed that there is no mediated effect of gender on reading competence of literary text when reading for entertainment. Yet, the results confirmed a significant indirect path from gender on both text-type-specific reading competences, mediated by the preference to read to gain information. We expected an indirect effect via the preference to read for information on reading competence in informational texts, but not on the reading competence in literary texts. Thus, the findings showed that adults' reading competence depended more on reading to gain information than reading for entertainment. However, men even caught up slightly to women in their reading competence by preferring to read non-fiction with the purpose to gain information. As a consequence, there was a small gender difference in the mediation model in favor of men in reading competence in informational texts after including preferences and control variables in the model. Simultaneously, these differentiated results of adults' reading preferences and the impact on text-type-specific 
reading competence could also be used to strengthen adolescence interests in reading informational texts.

The finding that reading for information is more important than reading for entertainment stresses the argument that it does not only matter what a reader reads. The preference to read for information might be more related to everyday life of adults than the preference to read for entertainment and with respect to heterogeneous topics and experiences. Since there are multiple functions of non-fictional expository literature (Groeben 2004), for example information reception, opinion formation, and knowledge acquisition, adult readers might be more encouraged to deal intensively with the topics of informational texts as compared to texts which should only entertain and serve as a withdrawal from everyday life. Additionally, the preference to read for information is also included in and associated with job-related reading. Rexing et al. (2015) found in a qualitative survey with adults that reading is more relevant for people in higher positions at the workplace (i.e., superiors compared to apprentices in the building trade) which also might be the case in the present sample of adults with high levels of qualifications. In future studies, the relevance of job-related reading of adults in different types of occupations as well as the distinction from and relation to leisure time reading should be considered more closely. In addition to the presumed relevance of job-related reading behavior for leisure time reading, the amount of reading on the job should also be measured in future studies. This might particularly be relevant since it is possible that individuals, who have to read a lot in their job, read less in their leisure time or seek different leisure time activities.

Moreover, Charlton et al. (2004) found in their study with adults that men search for additional information about what they were reading. Maybe this can also explain the small gender differences in favor of men in reading competence in informational texts. Therefore, in a further study with adults, it would be interesting to ask not only what exactly the preferences for a particular genre are, but also to examine the development of reading preferences for different genres. Additionally, this might also provide information to examine gender differences in reading competence further.

The current study provides an important theoretical contribution to the limited research on adults' reading competence. Nevertheless, the current study also has several limitations. First, the age composition of the sample is very heterogeneous, and the age of participants was not normally distributed due to an oversampling of university students in this study. In addition, there was also, on average, a very high level of education in the sample.

Second, the selection of texts to determine the text-type-specific reading competence was based on only nine texts. Artelt and Schlagmüller (2004) and Roick et al. (2013) showed that it is useful to distinguish between informational texts and literary texts. However, especially with respect to the literary texts, the larger variety of texts within this text type should be included in future studies. Additionally, it might also be interesting for future research to include other text types, for example, commentary or advertising texts as well as non-continuous texts. Similar to the selection of the textual content, there is a limitation in the differentiation of preferences. In future research, it would be advisable to divide the genre into smaller subcategories. A finer graduation of the genres might result in a differentiated effect with regard to reading preferences of women and men.

In conclusion, women and men showed in general different preferences for reading material in their leisure time. However, the different reading preferences of women and men did not explain gender differences in reading competence in literary texts. The gender differences were explained, albeit only slightly, in reading competence of informational texts and in favor of men by reading more often with the preference to gain information. Pfost et al. (2013) reported 
on a "virtuous circle of reading" which referred to the positive influence of reading experience on the development of reading competence and in turn on reading volume. This positive reading experience should be supported and promoted early in education so that the interest and meaning for reading maintained even in adulthood. The results of PISA showed that the overall interests for reading decline over the last PISA decade (Diedrich et al. 2019). This should be intervened already at school by offering a wide range of different reading topics and opportunities. The preferences for reading genres for boys and girls should be promoted in school, however, without enforcing some gender stereotypes by equally providing all topics to both genders.

Yet, the relationship of reading competence and reading preferences is complex; hence, the reciprocity and the direction of this relationship in various text types should be further investigated in future studies to determine the direction of the relationship.

Acknowledgements Open Access funding provided by Projekt DEAL.

Open Access This article is licensed under a Creative Commons Attribution 4.0 International License, which permits use, sharing, adaptation, distribution and reproduction in any medium or format, as long as you give appropriate credit to the original author(s) and the source, provide a link to the Creative Commons licence, and indicate if changes were made. The images or other third party material in this article are included in the article's Creative Commons licence, unless indicated otherwise in a credit line to the material. If material is not included in the article's Creative Commons licence and your intended use is not permitted by statutory regulation or exceeds the permitted use, you will need to obtain permission directly from the copyright holder. To view a copy of this licence, visit http://creativecommons.org/licenses/by/4.0/.

\section{References}

Adams, R. J., Wu, M. L., \& Wilson, M. R. (2015). ACER ConQuest: Generalised item response modelling software [computer software]. Version 4. Camberwell: Australian Council for Educational Research.

Allison, P. D. (2009). Missing data. Sage university papers quantitative applications in the social sciences: Vol. 136. Thousand Oaks: Sage Publ.

Artelt, C., McElvany, N., Christmann, U., Richter, T., Groeben, N., Köster, J., et al. (2007). Förderung von Lesekompetenz - Expertise [promotion of reading competence - an expertise]. Bildungsforschung: Vol. 17. Berlin: Bundesministerium für Bildung und Forschung.

Artelt, C., Naumann, J., \& Schneider, W. (2010). Lesemotivation und Lernstrategien [reading motivation and learning strategies]. In E. Klieme, C. Artelt, J. Hartig, N. Jude, O. Köller, M. Prenzel, et al. (Eds.), PISA 2009. Bilanz nach einem Jahrzehnt (pp. 73-112). Münster: Waxmann.

Artelt, C., Schiefele, U., \& Schneider, W. (2001). Predictors of reading literacy. European Journal of Psychology of Education, 16(3), 363-383. https://doi.org/10.1007/BF03173188.

Artelt, C., \& Schlagmüller, M. (2004). Der Umgang mit literarischen Texten als Teilkompetenz im Lesen? Dimensionsanalysen und Ländervergleiche [The handling of literary texts as aspect in reading? Dimensionality and comparison between countries]. In U. Schiefele, C. Artelt, W. Schneider, \& P. Stanat (Eds.), Struktur, Entwicklung und Förderung von Lesekompetenz. Vertiefende Analysen im Rahmen von PISA 2000 (pp. 169-196). Wiesbaden: VS Verlag für Sozialwissenschaften.

Baron, R. M., \& Kenny, D. A. (1986). The moderator-mediator variable distinction in social psychological research: Conceptual, strategic, and statistical considerations. Journal of Personality and Social Psychology, 51(6), 1173-1182.

Birnbaum, A. (1968). Some latent trait models and their use in inferring an examinee's ability. In F. M. Lord, M. R. Novick, \& A. Birnbaum (Eds.), Statistical theories of mental test scores (pp. 397-479). Reading: Addison-Wesley.

Blossfeld, H.-P., Roßbach, H.-G., \& von Maurice, J. (Eds.). (2011). Education as a lifelong process: The German National Educational Panel Study (NEPS). Zeitschrift für Erziehungswissenschaft/special issue: Vol. 14. Wiesbaden: VS Verlag für Sozialwissenschaften. 
Burbaum, C., Charlton, M., \& Schweizer, K. (2004). Wie und warum liest man Romane? Ergebnisse einer Telefonumfrage zur literarischen Lesekompetenz und zu den Lesestrategien von Erwachsenen [how and why do we read novels? Results of a telephone survey on adults' reading literacy and reading strategies]. Zeitschrift für Medienpsychologie, 16(2), 57-67.

Charlton, M., Burbaum, C., \& Sutter, T. (2004). Lesen Frauen wirklich anders? Oder lesen sie nur eine andere Literatur als Männer? [do women really read differently? Or do they just read a different type of literature than men?]. SPIEL, 23(1), 3-22.

Chiu, M. M., \& McBride-Chang, C. (2006). Gender, context, and reading: A comparison of students in 43 countries. Scientific Studies of Reading, 10(4), 331-362. https://doi.org/10.1207/s1532799xssr1004_1.

Christmann, U. (2004). Information als unmittelbare Funktion des Lesens [information as an immediate function of reading]. In N. Groeben \& B. Hurrelmann (Eds.), Lesesozialisation und Medien. Lesesozialisation in der Mediengesellschaft. Ein Forschungsüberblick (pp. 61-94). Beltz Juventa: Weinheim.

Curtis, R. F., \& Jackson, E. F. (1962). Multiple indicators in survey research. American Journal of Sociology, 68(2), 195-204.

Diamantopoulos, A., \& Riefler, P. (2008). Formative Indikatoren: Einige Anmerkungen zu ihrer Art, Validität und Multikollinearität [formative indicators: Some comments about their type, validity and multicollinearity]. Zeitschrift für Betriebswirtschaft, 78(11), 1183-1196. https://doi.org/10.1007/s11573008-0099-7.

Diedrich, J., Schiepe-Tiska, A., Ziernwald, L., Tupac-Yupanqui, A., Weis, M., McElvany, N., \& Reiss, K. (2019). Lesebezogene Schülermerkmale in PISA 2018: Motivation, Leseverhalten, Selbstkonzept und Lesestrategiewissen [reading-related student characteristics in PISA 2018: motivation, reading behavior, self-concept and reading strategy]. In K. Reiss, M. Weis, E. Klieme, \& O. Köller (Eds.), PISA 2018. Grundbildung im internationalen Vergleich (1st ed., pp. 80-110). Münster: Waxmann.

Enders, C. K., \& Bandalos, D. L. (2001). The relative performance of full information maximum likelihood estimation for missing data in structural equation models. Structural Equation Modeling: A Multidisciplinary Journal, 8(3), 430-457. https://doi.org/10.1207/S15328007SEM0803_5.

Gardner, D. (2004). Vocabulary input through extensive reading: A comparison of words found in children's narrative and expository reading materials. Applied Linguistics, 25(1), 1-37. https://doi.org/10.1093 /applin/25.1.1.

Gehrer, K., Zimmermann, S., Artelt, C., \& Weinert, S. (2013). NEPS framework for assessing reading competence and results from an adult pilot study. Journal for Educational Research Online, 5(2), 50-79.

Groeben, N. (2004). Einleitung: Funktionen des Lesens - Normen der Gesellschaft [introduction: Functions of reading - norms of the society]. In N. Groeben \& B. Hurrelmann (Eds.), Lesesozialisation und Medien. Lesesozialisation in der Mediengesellschaft. Ein Forschungsüberblick (pp. 11-35). Weinheim: Beltz Juventa.

Klieme, E., Artelt, C., Hartig, J., Jude, N., Köller, O., Prenzel, M.,. . Stanat, P. (Eds.). (2010). PISA 2009: Bilanz. nach einem Jahrzehnt [PISA 2009: Results after a decade]. Münster: Waxmann.

Lundetræ, K., Sulkunen, S., Gabrielsen, E., \& Malin, A. (2014). A comparison of PIAAC and PISA results. In A. Malin (Ed.), Associations between age and cognitive foundation skills in the Nordic countries: A closer look at the data (pp. 171-188). Jyväskylä: The Finnish Institute for Educational Research.

MacKinnon, D. P. (2013). Introduction to statistical mediation analysis. New York: Routledge.

Mar, R. A., \& Rain, M. (2015). Narrative fiction and expository nonfiction differentially predict verbal ability. Scientific Studies of Reading, 19(6), 419-433. https://doi.org/10.1080/10888438.2015.1069296.

McGeown, S. P., Duncan, L. G., Griffiths, Y. M., \& Stothard, S. E. (2015). Exploring the relationship between adolescent's reading skills, reading motivation and reading habits. Reading and Writing, 28(4), 545-569. https://doi.org/10.1007/s11145-014-9537-9.

Mol, S. E., \& Bus, A. G. (2011). To read or not to read: A meta-analysis of print exposure from infancy to early adulthood. Psychological Bulletin, 137(2), 267-296. https://doi.org/10.1037/a0021890.

Mullis, I. V. S., Martin, M. O., Foy, P., \& Drucker, K. T. (2012). PIRLS 2011 International results in reading. Chestnut Hill: TIMSS \& PIRLS International Study Center.

Muthén, L. K., \& Muthén, B. O. (2017). Mplus user's guide (8th ed.). Los Angeles: Muthén \& Muthén.

OECD. (1999). Measuring student knowledge and skills: A new framework for assessment. Paris: OECD Publishing.

OECD. (2013). The survey of adult skills: Reader's companion. Paris: OECD Publishing.

OECD. (2019). PISA 2018 results: What students know and can do. Paris: OECD Publishing.

Pfost, M., Dörfler, T., \& Artelt, C. (2013). Students' extracurricular reading behavior and the development of vocabulary and reading comprehension. Learning and Individual Differences, 26, 89-102. https://doi. org/10.1016/j.lindif.2013.04.008. 
Pohl, S., \& Carstensen, C. H. (2013). Scaling of competence tests in the National Educational Panel Study Many questions, some answers, and further challenges. Journal for Educational Research Online, 5(2), 189-216.

Reiss, K., Sälzer, C., Schiepe-Tiska, A., Klieme, E., \& Köller, O. (Eds.). (2016). PISA 2015: Eine Studie zwischen Kontinuität und Innovation [PISA 2015: A study between continuity and innovation. Münster: Waxmann.

Retelsdorf, J., Köller, O., \& Möller, J. (2011). On the effects of motivation on reading performance growth in secondary school. Learning and Instruction, 21(4), 550-559. https://doi.org/10.1016/j. learninstruc.2010.11.001.

Rexing, V., Keimes, C., \& Ziegler, B. (2015). Promotion of reading skills with recipient and domain specific focus - empirical findings in the professional field of construction engineering. Journal of Technical Education, 3(1), 56-74.

Roick, T., Frederking, V., Henschel, S., \& Meier, C. (2013). Literarische Textverstehenskompetenz bei Schülerinnen und Schülern unterschiedlicher Schulformen [literary text comprehension of students in different school types]. In C. Rosebrock (Ed.), Literalität erfassen. Bildungspolitisch, kulturell, individuell (pp. 69-84). Weinheim: Beltz Juventa.

Rost, J. (2004). Lehrbuch Testtheorie - Testkonstruktion [textbook test theory - test construction (2nd, extended and rev. ed.). Bern: Huber.

Schiefele, U., Schaffner, E., Möller, J., \& Wigfield, A. (2012). Dimensions of reading motivation and their relation to reading behavior and competence. Reading Research Quarterly, 47(4), 427-463. https://doi. org/10.1002/RRQ.030.

Schreier, M., \& Odağ, Ö. (2004). Genuss und Distanz: Geschlechtsspezifische Kompetenzen beim Umgang mit Realität(en) und Fiktion(en) [gender-specific competencies at dealing with fiction and nonfiction]. SPIEL, 23(1), 94-115.

Solheim, O. J., \& Lundetræ, K. (2017). Can test construction account for varying gender differences in international reading achievement tests of children, adolescents and young adults? - a study based on Nordic results in PIRLS, PISA and PIAAC. Assessment in Education: Principles, Policy \& Practice, 3 , 1-20. https://doi.org/10.1080/0969594X.2016.1239612.

Stanat, P., \& Kunter, M. (2002). Geschlechterspezifische Leistungsunterschiede bei Fünfzehnjährigen im internationalen Vergleich [an international comparison of gender-specific differences in achievement of 15-year-olds]. Zeitschrift für Erziehungswissenschaft, 4(1), 28-48. https://doi.org/10.1007/s11618-0020003-0.

Stiftung Lesen (2008). Lesen in Deutschland 2008 [Reading in Germany 2008], Mainz.

Torppa, M., Niemi, P., Vasalampi, K., Lerkkanen, M.-K., Tolvanen, A., \& Poikkeus, A.-M. (2019). Leisure reading (but not any kind) and reading comprehension support each other - a longitudinal study across grades 1 and 9. Child Development, 1-25. https://doi.org/10.1111/cdev.13241.

Weiber, R., \& Mühlhaus, D. (2014). Strukturgleichungsmodellierung: Eine anwendungsorientierte Einführung in die Kausalanalyse mit Hilfe von AMOS, SmartPLS und SPSS [structural equation modelling: An applied introduction to causal analysis using AMOS SmartPLS and SPSS (2nd, extended and rev. ed.). Berlin: Springer Gabler.

Weinert, F. E. (2001). Concept of competence: A conceptual clarification. In D. S. Rychen \& L. H. Salganik (Eds.), Defining and selecting key competencies (pp. 45-66). Seattle: Hogrefe and Huber.

Kathrin Thums. Leibniz Institute for Educational Trajectories, Wilhelmsplatz 3, 96047 Bamberg, Germany. Email: kathrin.thums@lifbi.de

Current themes of research:

Reading competence. Competence development. Gender differences in reading competence.

Most relevant publications in the field of Psychology of Education:

No publications yet 
Cordula Artelt. Leibniz Institute for Educational Trajectories, Wilhelmsplatz 3, 96047 Bamberg, Germany.

Current themes of research:

Text comprehension. Reading competence and competence assessment.

Most relevant publications in the field of Psychology of Education:

Artelt, C. (2016). Teacher judgments and their role in the educational process. Emerging Trends in the Social and Behavioral Sciences: An Interdisciplinary, Searchable, and Linkable Resource, 1-16. Wiley. DOI: 10.1002/ 9781118900772.etrds0402

Edossa, A. K., Neuenhaus, N., Artelt, C., Lingel, K. \& Schneider, W. (2018 online). Developmental Relationship between declarative metacognitive knowledge and reading comprehension during secondary school. European Journal of Psychology of Education, 1-20. DOI: 10.1007/s10212-018-0393-x

Pfost, M., Hattie, J., Dörfler, T. \& Artelt, C. (2014, online first). Individual differences in reading development: reviewing 25 years of empirical research on Matthew effects in reading. Review of Educational Research, 84 (2), 203-244. DOI: 10.3102/0034654313509492

Weinert, S., Artelt, C., Prenzel, M., Senkbeil, M., Ehmke, T., Carstensen C.H. \& Lockl, K. (2019). Development of competencies across the life course. In H. P. Blossfeld \& H.-G. Roßbach (Hrsg.), Education as a Lifelong Process (2. Auflage). (S. 57 - 81). Wiesbaden: Springer.

Ilka Wolter. Leibniz Institute for Educational Trajectories, Wilhelmsplatz 3, 96047 Bamberg, Germany.

Current themes of research:

Self-concept and academic achievement. Gender stereotypes and identity. Competence development. Genderspecific learning environments. Reading competence and text comprehension.

Most relevant publications in the field of Psychology of Education:

Ehrtmann, L., \& Wolter, I. (2018). The impact of students' gender-role orientation on competence development in mathematics and reading in secondary school. Learning and Individual Differences, 61, 256-264. doi: 10.1016/j.lindif.2018.01.004

Wolter, I., \& Hannover, B. (2016). Gender role self-concept at school start and its impact on academic selfconcept and performance in mathematics and reading. European Journal of Developmental Psychology, 13, 681-703. doi: 10.1080/17405629.2016.1175343

Bieg, M., Götz, T., Wolter, I., \& Hall, N. C. (2015). Gender stereotype endorsement differentially predicts girls' and boys' trait-state discrepancy in math anxiety. Frontiers in Psychology, 6: 1404. doi: 10.3389/ fpsyg.2015.01404

Wolter, I., Braun, E., \& Hannover, B. (2015). Reading is for girls!? The negative impact of preschool teachers' traditional gender role attitudes on boys' reading related motivation and skills. Frontiers in Psychology, 6: 1267. doi: 10.3389/fpsyg.2015.0126

Publisher's note Springer Nature remains neutral with regard to jurisdictional claims in published maps and institutional affiliations. 
Beitrag 3

Thums, K., Gnambs, T., \& Wolter, I. (2020). The impact of gender-stereotypical text contents on reading competence in women and men. Zeitschrift für Erziehungswissenschaft, 23(6), 1283-1301. doi.org/10.1007/s11618-020-00980-8 


\title{
The impact of gender-stereotypical text contents on reading competence in women and men
}

\author{
Kathrin Thums (D) Timo Gnambs • Ilka Wolter
}

Received: 26 November 2019 / Revised: 19 June 2020 / Accepted: 13 October 2020

(C) The Author(s) 2020

\begin{abstract}
Societies have socially shared assumptions about what constitutes typically male or female attributes. Language can contribute to gender inequality by transmitting gender stereotypes. This study examines whether gender-stereotypical connotations in stimulus texts within a reading competence test might serve as a nuisance factor distorting reading competence measurements. In addition to a general factor for reading competence, we expected gender-stereotypical texts to give rise to gender-specific factors regarding the text content. The research was based on a sample of 813 adults from a pilot study of the German National Educational Panel Study (NEPS). A bifactor model confirmed a general factor for reading competence. However, the two gender-specific factors were not found; consequently, no substantial gender differences in reading competence for gender-stereotypical text content were observed. These findings indicate that there is no substantial impact of gender-stereotypical text connotations on the measurement of women's and men's reading competence.
\end{abstract}

Electronic supplementary material The online version of this article (https://doi.org/10.1007/ s11618-020-00980-8) contains supplementary material, which is available to authorized users.

K. Thums $(\triangle)$

Leibniz Institute for Educational Trajectories, Wilhelmsplatz 3, 96047 Bamberg, Germany

E-Mail: kathrin.thums@tu-dortmund.de

K. Thums · Prof. Dr. T. Gnambs · Dr. I. Wolter

Institut für Schulentwicklungsforschung (IFS), Technische Universität Dortmund,

Vogelpothsweg 78, 44227 Dortmund, Germany

Prof. Dr. T. Gnambs

E-Mail: timo.gnambs@lifbi.de

Dr. I. Wolter

E-Mail: ilka.wolter@lifbi.de 
Keywords Gender differences - Reading competence - Stereotype text content · Text characteristics

\section{Der Einfluss von geschlechtsstereotypen Textinhalten auf die Lesekompetenz von Frauen und Männern}

Zusammenfassung Es gibt sozial geteilte Annahmen darüber, was typisch weibliche oder männliche Eigenschaften sind oder sein sollten. Über Sprache werden Stereotype vermittelt, beispielsweise über geschlechtsstereotype Textinhalte, wodurch Ungleichheiten zwischen Frauen und Männern aufrechterhalten werden. Die vorliegende Studie untersucht, ob geschlechtsstereotype Konnotationen von Texten, die zur Messung der Lesekompetenz verwendet werden, einen differenziellen Einfluss für Frauen und Männer auf das Ergebnis der Messung haben. Neben einem generellen Faktor der Lesekompetenz wurde erwartet, dass sich geschlechterspezifische Faktoren identifizieren lassen. Die Stichprobe umfasste 813 Erwachsene aus einer Entwicklungsstudie des Nationalen Bildungspanels (NEPS). Im Bi-Faktormodell konnte der allgemeine Faktor der Lesekompetenz bestätigt werden. Darüber hinaus konnten die beiden geschlechtsspezifischen Faktoren jedoch nicht ermittelt werden. Es zeigten sich keine Unterschiede in der Lesekompetenz hinsichtlich geschlechtsstereotyper Textinhalte. Das Ergebnis deutet darauf hin, dass eine geschlechtsstereotype Textkonnotation keinen substantiellen Einfluss auf die Lesekompetenzmessung von Frauen und Männern hat.

Schlüsselwörter Lesekompetenz - Geschlechterunterschiede · Textmerkmale · Stereotype Textinhalte

\section{Research background and aims}

\subsection{Reading competence and gender differences in reading competence}

A broad range of diverse competences are necessary for active participation in social life. The focus of this study is on reading competence, a fundamental competence for participation in social life among all age groups (OECD 2013b, 2016b). Reading comprehension is described as the product of characteristics of the reader (e.g., gender, prior knowledge, and motivation) and characteristics of texts and tasks (e.g., text content, coherence; cf. Artelt et al. 2001; Christmann and Groeben 1999). One form of this interaction between reader characteristics (gender) and text characteristics (gender-stereotypical text content) was investigated in this study as a potential explanation for gender differences in reading competence between women and men.

The ability to read and understand written language is referred to as reading competence. Reading competence is rooted in cognitive representations and processes and can be learned and trained (Müller and Richter 2014). According to Graesser et al. (1994), reading competence goes beyond the mere decoding of letters, words, and sentences. Rather, this functional and active approach to reading competence emphasizes how a reader engages with the content of a text, obtains information 
from the text, and thus draws inferences and places the text content within the global coherence of everyday life (Graesser et al. 1994). Kintsch's (1998) definition of reading competence stresses the interaction between readers' features and text characteristics while emphasizing the active role of the reader, who must integrate prior knowledge and a mental representation of the text during the reading process. In contrast, the current paper mainly relies on the functional literacy definition of reading competence, in which the focus is on the resulting product, i.e., the quality of an individual's text representation (Müller and Richter 2014). Consequently, no statements will be made about individual cognitive processes involved in reading.

Reading competence assessments have revealed gender differences throughout school. The results generally suggest that girls achieve higher reading competence scores than boys (e.g., Berendes et al. 2018; Solheim and Lundetræ 2017; Weis et al. 2019), with the largest gender differences in reading during secondary school. In all previous PISA (Programme for International Student Assessment) cycles, 15year-old girls outperformed boys in reading competence on OECD average (OECD 2019). In contrast, in the PIAAC study (Programme for the International Assessment of Adult Competencies), there were no substantial competence differences between women and men both on OECD average and for the German subsample (OECD 2016a; Solheim and Lundetræ 2017). However, there is much less research on adults' reading competence compared to younger cohorts.

Thus, while previous studies found no substantial gender differences in overall reading competence among adult readers (OECD 2016a), the present study conducts a more differentiated analysis of men's and women's reading competence in interaction with text characteristics, specifically gender-stereotypical text content.

\subsection{The role of gender stereotypes in explaining gender differences in reading competence}

As previously mentioned, reading comprehension is described as an interaction between readers' characteristics and features of texts or tasks (cf. Artelt et al. 2001; Christmann and Groeben 1999). The present study aimed to further investigate gender differences in reading competence based on a specific form of this proposed interaction between readers' characteristics (i.e., gender) and text characteristics (i.e., gender-stereotypical text content). We expect that men and women process texts with gender-stereotypical text content differently. More precisely, we expect that men have a higher reading competence in texts with stereotypically male connotations than women, who in turn are expected to be superior in reading texts with stereotypically female connotations.

Evidence for this research question can be derived from various research paradigms: The expectancy-value theory (e.g., Eccles 1987; Wigfield et al. 2006), adapted for reading motivation by Möller and Schiefele (2004), provides a potential explanation for gender differences in reading competence. The central components of this theory influencing behavior and motivation, which in turn affect one's competences over the life course (e.g., Becker and McElvany 2018), are expectation beliefs regarding success and failure and subjective value beliefs. According to this theory, women should have lower expectations of success regarding stereotypically 
male text contents and consider those contents less important or relevant (i.e., lower value) than men, who in turn should hold lower expectations of success and lower value than women towards stereotypically female content domains.

Individuals' values and expectations are in turn influenced by their social environment. For example, one's social environment includes one's socio-economic status, cultural habits, and experiences with reading, but also gender roles and cultural stereotypes concerning specific academic domains and occupations (Becker and McElvany 2018; Möller and Schiefele 2004). Since gender stereotypes and gender roles are transmitted through the social environment, they are expected to have impact on individuals' interests (values) and self-concept (expectancy) (Möller and Schiefele 2004). Therefore, another possible explanation for gender differences in reading competence is a lower reading-related self-concept-as a consequence of a lower expectation of success-towards stereotypically male text contents in women relative to men, and a lower reading-related self-concept towards stereotypically female text contents in men relative to women. It is plausible to assume that gender stereotypes influence individuals' motivation and interest in specific topics. Since referring a high value to a particular topic leads one to have positive associations with it, which can in turn be an important source of motivation for continuing this activity (Möller and Schiefele 2004), it is important to investigate the impact of gender stereotypes in the context of reading competence.

Gender stereotypes are defined as associating certain characteristics, attitudes, abilities, preferences, and behavior to women and men (Athenstaedt 2003; Prentice and Carranza 2002). Stereotypes are not attributions made about individuals but rather a socially shared assumption regarding the social group in general. Gender stereotypes have both a descriptive (how women or men typically are) and a prescriptive component (how women or men are expected to be) (Heilman 2012; Prentice and Carranza 2002).

Some of the most common gender stereotypes include that men are (expected to be) agentic, confident, competitive, independent, and assertive (e.g., Diekman and Eagly 2000; Fiske et al. 2002). Other gender stereotypes are that women are (expected to be) affectionate, considerate, warm, and communal (e.g., Diekman and Eagly 2000; Fiske et al. 2002). In addition to these personality traits ascribed to women and men, there are gender stereotypes concerning leisure time activities and occupations (Athenstaedt 2003; Baron et al. 2001; Nosek et al. 2002). Leadership and management positions as well as interest in activities and occupations in STEM fields (science, technology, engineering, and mathematics) are generally perceived as the domains of men (Baron et al. 2001; Nosek et al. 2002). Conversely, social activities, taking care of children, and activities and occupations in the arts, language and social science are gender stereotypes associated with women (Baron et al. 2001; Nosek et al. 2002). Furthermore, research has repeatedly demonstrated achievementrelated stereotypes: boys are more interested and achieve higher performance in mathematics (e.g., Nowicki and Lopata 2017; Steffens and Jelenec 2011). However, reading is generally considered a female domain and associated activities are often seen as female-typed (e.g., Nowicki and Lopata 2017; Steffens and Jelenec 2011; Watson et al. 2010). Consequently, there are robust gender differences between women and men with regard to interest in reading and perceived value of reading. 
Girls have a higher intrinsic reading motivation and read more frequently on average (Logan and Johnston 2009; McGeown et al. 2012; Schiefele et al. 2012). Likewise, girls' attitudes towards reading are more positive than boys' attitudes (Logan and Johnston 2009).

If these robust and socially shared expectations of women and men (i.e., gender stereotypes) are also reflected in the content of reading material within a reading competence test, gender stereotypes may affect the assessment of men's and women's reading competence. Previous research shows that gender stereotypes are perpetuated and transmitted through formal and semantic features of language in texts as well as text content. Hence, texts' language and content also contribute to enhancing and maintaining gender stereotypes fixed within oral and written language (Garnham et al. 2016).

Various studies have shown that gender differences in overall reading competence can be explained by certain characteristics of the administered instruments. For example, gender differences in reading are affected by the item format: Boys struggle more with open-ended questions compared to girls. Specifically, across several PISA studies, 15-year-old boys experienced more problems with open formats, with their answers containing less or irrelevant information compared to those of girls (e.g., Lafontaine and Monseur 2009; Schwabe et al. 2015). Further studies confirmed that girls have an advantage in continuous text formats and achieve higher reading competence (Artelt and Schlagmüller 2004; Stanat and Kunter 2002).

Studies on the hierarchical lower level of words instead of sentences or texts found qualitative differences between girls and boys in a vocabulary test (McElvany and El-Khechen 2015; McElvany et al. 2016). Girls and boys more often knew words consistent with stereotypes associated with their own gender than words associated with the other gender group.

These results indicate that examining text characteristics might be relevant for explaining gender differences in reading competence. However, no research currently exists on whether the text context, specifically gender-stereotypical content, has an impact on men's and women's reading competence. Therefore, the present study investigated the impact of gender-stereotyped text content in a large-scale assessment of reading competence, extending previous studies by examining differences at the level of longer and more complex texts rather than words or individual sentences (cf. McElvany and El-Khechen 2015; McElvany et al. 2016). The gender-stereotypicality of texts was expected to explain gender differences in reading competence based on the assumption that, in general, women and men have different interests and prior knowledge based on different experiences in their social environments (e.g., education, occupation, leisure time activities; Baron et al. 2001; Christin 2012; Lagaert et al. 2017; Nosek et al. 2002). Women's and men's different levels of interest, previous experiences, and prior knowledge in regard to text contents with gender-stereotypical connotations might initiate that they feel competent and familiar in dealing with different text contents. Consequently, women and men are expected to achieve different competence levels in a reading competence assessment when the text content includes gender stereotypes. 


\subsection{Research aims and hypotheses}

Drawing upon this theoretical and empirical foundation, we hypothesized that gender differences in reading competence can be explained by gender-stereotypical connotations in text content. It is plausible to assume that different levels of prior knowledge and relevant previous experience in different contexts lead to higher familiarity with the text content and therefore make it easier to link information from texts, draw inferences and build coherence with other content. Combined with their aforementioned higher interest in gender-stereotypical domains, this might explain why competence levels in a reading competence test depend on the genderstereotypical content of the text.

Consequently, men are expected to outperform women in a text whose content addresses, for example, a technical topic, which is a stereotypically male domain (Diekman and Eagly 2000; Heilman 2012). Conversely, women are expected to outperform men on a text with content, for example, about a love story or human relationships, due to the stereotype that women are more emotionally invested in stories and interested in communion (Diekman and Eagly 2000; Heilman 2012).

The hypotheses were as follows:

Hypothesis 1 Alongside a general factor for reading competence, with substantial factor loadings for all tasks on one unidimensional scale, there should be two additional gender-specific factors for reading competence. These gender-specific factors reflect a) stereotypically female text content; and b) stereotypically male text content.

Hypothesis 2 Conditional on the support of hypothesis 1, we expect gender differences in the gender-specific factors for reading competence: a) women have higher reading competence on stereotypically female texts than men, and b) men have higher reading competence on stereotypically male texts than women.

Hence, the hypotheses refer to different levels of analyses. The first hypothesis refers to the material for assessing reading competence, which was tested for further dimensions of reading competence based on gender stereotypes in text content. The second hypothesis refers to interindividual differences (i.e., gender differences) in these specific factors for reading competence. Thus, this second hypothesis relates to the interaction between readers' characteristics (gender) and text characteristics (gender-stereotypical text content).

\section{Methods}

\subsection{Sample and design}

The data was collected within a pilot study of the German National Educational Panel Study (Blossfeld et al. 2011). The sample was a stratified random sample and predefined using a quota scheme with regard to age and education.

Reading competence was tested among a sample of 939 adults using computerbased assessments in their own households. A subsample of $n=39$ participants 
Table 1 Sample distribution by age and highest level of school education

\begin{tabular}{lll}
\hline & $N$ & Percent \\
\hline Age & & \\
19-25 years & 478 & 58.8 \\
26-45 years & 219 & 26.9 \\
46-65 years & 112 & 13.8 \\
Not specified & 4 & 0.5 \\
\hline Highest level of school education & & \\
Did not complete any form of schooling & 2 & 0.2 \\
Special education school leaving certificate & 5 & 0.6 \\
Basic Hauptschule leaving certificate & 57 & 7.1 \\
Qualifying Hauptschule leaving certificate & 36 & 4.4 \\
Realschule leaving certificate & 134 & 16.5 \\
University of applied sciences entrance qualification (Fach- & 54 & 6.6 \\
holschulreife) & & \\
University entrance qualification (Abitur) & 525 & 64.6 \\
\hline
\end{tabular}

who were assessed in a different test condition as part of the experimental design were excluded. Since only a few participants came from an immigrant background and had a first language other than German $(n=66)$, these participants were also excluded from the analyses. Moreover, all participants born before $1950(n=21)$ were excluded from the analyses because we expected different gender attributions and gender stereotypes in older cohorts (Athenstaedt 2000; Eagly et al. 2019; Haines et al. 2016).

Thus, the final sample consisted of 813 adults, of which $n=424$ were female (52.2\%). Their ages ranged from 19 to 65 years old $(M=30.08 ; S D=11.16)$. A detailed description of the sample distribution in terms of age and school education is displayed in Table 1. Supplementary information about the sample distribution regarding gender, age, and school education is available at: https://osf.io/h8m7a/.

\subsection{Research instruments}

\subsubsection{Reading competence}

The reading competence test was based on a consistent framework (Gehrer et al. 2012 , 2013) identifying five different text types: informational texts, commentaries or argumentative texts, literary texts, instructional texts, and advertising texts. The pilot study was conducted using a multi-matrix-design with overall 20 text of which participants were randomly assigned to 15 texts and the associated tasks. For this study six informational texts, three literary texts and three advertising texts were included in total in the analyses for the present study. The test consisted of 68 dichotomous multiple-choice tasks as well as more complex polytomous multiplechoice tasks (e.g., ordering tasks, and cloze tasks) related to the content of the respective texts. The tasks posed different cognitive requirements on the reader: 
finding information in the text, drawing text-related conclusions (local and global coherence information), and engaging in reflection and assessment.

\subsubsection{Background variable gender}

Participants indicated their gender in the study questionnaire as either male or female. In the analyses, gender was coded 0 for female and 1 for male.

\subsection{Data analysis}

\subsubsection{Rating and scaling the reading texts}

The twelve texts assessing reading competence were categorized a priori with regard to content into texts with a gender-stereotypically male connotation, texts with a gender-neutral connotation and texts with a gender-stereotypically female connotation. This categorization took place purely based on the overall content of each individual text.

The categorization of texts into gender-stereotypically male, gender-stereotypically female and gender-neutral was conducted in two steps. The first step was to derive characteristics from the psychological and educational literature describing stereotypically female and male attributes, characteristics, interests, occupations, and activities based on previous empirical research (Athenstaedt 2003; Athenstaedt et al. 2009; Baron et al. 2001; Christin 2012; Fiske et al. 2002; Haines et al. 2016; Nosek et al. 2002). In the next step, three experts on the topic of gender stereotypes (two of whom are authors of this text) classified the twelve texts with regard to content with gender-stereotypically male, gender-stereotypically female or gender-neutral connotations. Their ratings concerned a text's overall topic on a continuous response scale with five options: very typically male, somewhat typically male, gender-neutral, somewhat typically female, and very typically female. The rating scheme can be found in the appendix. An example of stereotypically female content is a text about yoga and different yoga styles. Yoga is a typically female sport, and more women are interested in and familiar with different yoga traditions then men. An example of stereotypically male content is a text about a photography technique with a lot of technical details about the camera and the underlying technical procedure (Athenstaedt et al. 2009; Christin 2012; Lagaert et al. 2017).

Following the rating procedure, each gender-stereotypical text cluster contained four texts (two informational texts, one literary text and one advertising text). The experts achieved very high levels of consensus according to interrater reliability as measured through the intra-class-correlation, $I C C=0.86$.

After categorizing of the texts into gender-stereotypical text clusters, the 68 tasks referring to the texts were scaled with a two-parametric logistic item response model (2PL; Birnbaum 1968) using the software package ConQuest (version 4.5.2; Adams et al. 2015). The tasks were scaled separately for each gender-stereotypical text content category. This resulted in three separate scaling models, each on a logit scale. In this way, three well-fitting, unidimensional tests conforming to the $2 \mathrm{PL}$ model were developed. 
Table 2 Characteristics of gender-stereotyped and neutral texts

\begin{tabular}{lll}
\hline $\begin{array}{l}\text { Gender-stereotypical female } \\
\text { texts }\end{array}$ & Gender-neutral texts & Gender-stereotypical male texts \\
\hline 1016 words & 1162 words & 1028 words \\
23 tasks $(13 \mathrm{MC}, 10 \mathrm{CMC})$ & 21 tasks $(11 \mathrm{MC}, 10 \mathrm{CMC})$ & 24 tasks $(12 \mathrm{MC}, 12 \mathrm{CMC})$ \\
Marginal reliability $=0.70$ & Marginal reliability $=0.66$ & Marginal reliability $=0.73$ \\
Item difficulty $M=-0.97$ & Item difficulty $M=-0.18$ & Item difficulty $M=-0.75$ \\
$(S D=1.06)$ & $(S D=0.92)$ & $(S D=1.12)$ \\
\hline
\end{tabular}

$M C$ dichotomous multiple choice, $C M C$ polytomous complex multiple choice

Tasks were selected based on their quality (i.e., weighted mean square error, item characteristic curve; Pohl and Carstensen 2013) and discrimination parameters (cf. Rost 2004). Moreover, a differential item functioning (DIF) analysis between men and women was conducted. With the DIF analysis we validated the functions of the single tasks for women and men when controlling for the same reading competence. According to the NEPS scaling guideline, differences between groups of 0.40 to 0.60 represent considerable but not severe cases of DIF, and differences smaller than 0.40 are considered non-considerable DIF (Pohl and Carstensen 2013). All 68 tasks exhibited a DIF below 0.60, which was considered as negligible and the differences were in both directions (i.e., in favor of both genders).

Table 2 compares the three gender-stereotypical text clusters in terms of different indicators after the scaling and DIF analysis. For example, texts in the three categories were comparable in terms of the number of words and tasks. Likewise, the reliability scores were similar and satisfactory for all three categories. The item difficulties for stereotypically male and female texts were very similar. The average item difficulties for tasks with a gender-neutral text connotation was slightly higher $(M=-0.18, \mathrm{SD}=0.92)$ than for tasks in the other two text categories (genderstereotypical female texts $M=-0.97, S D=1.06$ and gender-stereotypical male texts $M=-0.75, S D=1.12$ ). Particularly, the two gender-stereotypical tests did not differ substantially in their average difficulties; thus, differences in item difficulties are unlikely to bias our analyses (see also footnote on the robustness check on page 8).

\subsubsection{Statistical models}

A confirmatory ordinal bifactor model was applied to examine the first hypothesis concerning text dimensionality. The bifactor model tested the dimensional structure of the tasks with a latent structural model containing one general factor and two additional group factors. A general factor addresses the target construct (e.g., reading competence) and has a conceptually broader alignment. Therefore, all 68 applied tasks were expected to have substantial loadings $(\beta \geq 0.40)$ on the general factor of reading competence. In addition to the general factor, we expected to find group factors representing subdomain constructs (e.g., gender-stereotypical text connotations). Thus, the bifactor model aimed to estimate how much variance is attributable to the general factor and how much to the group factors. The group factors should explain additional specific variance beyond the global factor (Chen et al. 2012; Rodriguez et al. 2016). Because of the large age span in the sample (19 to 65 years old), 


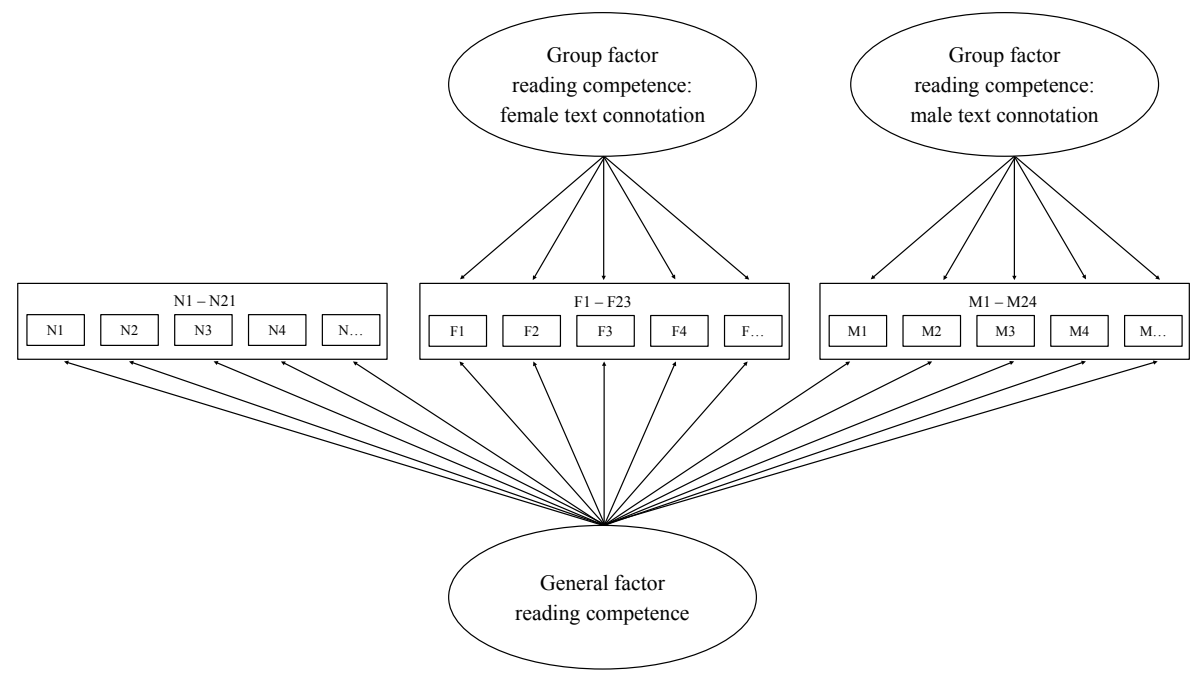

Fig. 1 Hypothesized bifactor model for reading competence with $N=$ tasks with gender-neutral text connotation; $F=$ tasks with female text connotation; $M=$ tasks with male text connotation

we controlled for age differences by regressing all observed indicators and latent variables on the age of the respondents. As a result, the latent factor loadings and variances are corrected for age-related differences. All analyses were also repeated without control variables; but these yielded highly similar results (see supplement).

Fig. 1 illustrates the hypothesized model. Tasks F1 to F23 were expected to have substantial factor loadings on the stereotypically female group factor for reading competence. Tasks M1 to M24 were expected to have factor loadings on the stereotypically male group factor for reading competence. The gender-neutral text connotation (Tasks N1-N21) represented the reference category in this model. Since the factors were set to be orthogonal to each other, there were no correlations among the factors (Reise 2012). The estimator used for the analysis was a maximum likelihood estimation with robust standard errors. The model was implemented in Mplus (version 8; Muthén and Muthén 2017). The Mplus syntax with model input- and output is available at: https://osf.io/h8m7a/.

Afterwards, a multiple group comparison (Kleinke et al. 2017) was computed to test for interindividual differences between women and men in the specific factors for reading competence, as assumed in the second hypothesis. Thus, the average reading competence among women was compared to the average reading competence among men for each factor. In the model, the factor loadings and item intercepts for all reading competence factors were set equal between women and men. The means and standard deviations for the two gender-stereotypical competence factors among women were freely estimated and compared to the means among men. This analysis was also conducted in Mplus (version 8; Muthén and Muthén 2017). 


\section{Results}

\subsection{Bifactor model}

The results for the bifactor model are summarized in Table 3. The first column shows the standardized loadings for the general factor for reading competence. All 68 tasks loaded significantly $(p<0.05)$ onto the general factor. Most tasks (41 tasks) had noteworthy factor loadings $>0.40$. The median factor loading for all tasks for the general factor was $M d n=0.48$. Reading competence was therefore measured with a general factor.

In contrast, the two gender-stereotypical group factors for reading competence were not confirmed; these findings are illustrated in the second and third columns of Table 3. For both group factors, most loadings were mostly below 0.40 . The median factor loading for stereotypically male text group factor was $M d n=0.11$, and the median factor loading for the stereotypically female text group factor was $M d n=0.29$. For both group factors, there were no substantial loadings or variances beyond the general factor. The results confirmed that a unidimensional model explained the empirical data best. ${ }^{1}$

In addition to the bifactor model results indicating a lack of specific factors for reading competence, we compared the fit of a unidimensional model that included only the general factor (but specific factors) with that of bifactor model. In the bifactor model, the Akaike information criterion (AIC) was 48,710 and the Bayesian information criterion (BIC) was 50,230. However, in the unidimensional factor model, the AIC was 48,778 and the BIC was 50,018. The AIC showed a better fit for the bifactor model, albeit with a rather small difference $(\triangle \mathrm{AIC}=8)$. In contrast, the BIC that also takes the number of estimated parameters into account preferred the unidimensional model. Thus, at least with respect to the BIC, the model fit confirmed the validity of a unidimensional factor model.

To check whether the results depend conditionally on the age of the participants, we examined the model without the age. Similar results were shown and also the unidimensional factor model would be the better solution. All results and the model comparison are available under: https://osf.io/h8m7a/.

Consequently, we cannot confirm Hypothesis 1. In contrast to our hypothesis, there were no additional specific factors for reading competence regarding genderstereotypical content in the text material.

\subsection{Multiple group comparison}

To test the second hypothesis at the individual level, a multiple group comparison of the bifactor model was conducted. There were no significant gender differences in reading competences in gender-stereotypical male connotation $(M=-0.19$ logits, $S D=0.19, p=0.31 ; d=-0.07)$.

\footnotetext{
1 As a robustness check, we reran the model separately for easy (item difficulty $<0$ ) and difficult (item difficulty $>0$ ) tasks in the reading competence test and could not identify specific factors with regard to the gender-stereotypical text content. The output is available at: https://osf.io/h8m7a/.
} 
Table 3 Standardized loading pattern for the bifactor model

\begin{tabular}{|c|c|c|c|c|c|c|}
\hline \multirow[t]{2}{*}{$\begin{array}{l}\text { Task } \\
\#\end{array}$} & \multicolumn{2}{|c|}{ General factor } & \multicolumn{2}{|c|}{$\begin{array}{l}\text { Group factor for } \\
\text { gender-stereotyped female text }\end{array}$} & \multicolumn{2}{|c|}{$\begin{array}{l}\text { Group factor for } \\
\text { gender-stereotyped male text }\end{array}$} \\
\hline & $B$ & S.E. & $B$ & S.E. & $B$ & S.E. \\
\hline N1 & 0.36 & 0.06 & - & - & - & - \\
\hline $\mathrm{N} 2$ & 0.52 & 0.05 & - & - & - & - \\
\hline N3 & 0.42 & 0.07 & - & - & - & - \\
\hline N4 & 0.24 & 0.05 & - & - & - & - \\
\hline N5 & 0.25 & 0.05 & - & - & - & - \\
\hline N6 & 0.37 & 0.05 & - & - & - & - \\
\hline N7 & 0.52 & 0.05 & - & - & - & - \\
\hline N8 & 0.42 & 0.06 & - & - & - & - \\
\hline N9 & 0.45 & 0.06 & - & - & - & - \\
\hline N10 & 0.41 & 0.05 & - & - & - & - \\
\hline N11 & 0.49 & 0.05 & - & - & - & - \\
\hline N12 & 0.31 & 0.06 & - & - & - & - \\
\hline N13 & 0.41 & 0.05 & - & - & - & - \\
\hline N14 & 0.27 & 0.05 & - & - & - & - \\
\hline N15 & 0.57 & 0.04 & - & - & - & - \\
\hline N16 & 0.37 & 0.05 & - & - & - & - \\
\hline N17 & 0.45 & 0.05 & - & - & - & - \\
\hline N18 & 0.31 & 0.05 & - & - & - & - \\
\hline N19 & 0.59 & 0.03 & - & - & - & - \\
\hline $\mathrm{N} 20$ & 0.48 & 0.04 & - & - & - & - \\
\hline $\mathrm{N} 21$ & 0.72 & 0.03 & - & - & - & - \\
\hline $\mathrm{F} 1$ & 0.35 & 0.06 & -0.02 & 0.22 & - & - \\
\hline $\mathrm{F} 2$ & 0.53 & 0.06 & -0.02 & 0.13 & - & - \\
\hline F3 & 0.30 & 0.06 & 0.12 & 0.21 & - & - \\
\hline F4 & 0.48 & 0.05 & 0.16 & 0.18 & - & - \\
\hline F5 & 0.26 & 0.06 & 0.07 & 0.13 & - & - \\
\hline F6 & 0.33 & 0.05 & -0.03 & 0.17 & - & - \\
\hline F7 & 0.40 & 0.05 & 0.30 & 0.19 & - & - \\
\hline F8 & 0.50 & 0.05 & 0.18 & 0.17 & - & - \\
\hline F9 & 0.41 & 0.05 & 0.20 & 0.16 & - & - \\
\hline F10 & 0.55 & 0.05 & -0.11 & 0.19 & - & - \\
\hline F11 & 0.53 & 0.05 & -0.10 & 0.20 & - & - \\
\hline $\mathrm{F} 12$ & 0.50 & 0.05 & -0.15 & 0.19 & - & - \\
\hline F13 & 0.41 & 0.06 & 0.23 & 0.11 & - & - \\
\hline F14 & 0.71 & 0.05 & 0.00 & 0.12 & - & - \\
\hline F15 & 0.34 & 0.06 & -0.10 & 0.14 & - & - \\
\hline F16 & 0.59 & 0.06 & 0.74 & 0.28 & - & - \\
\hline F17 & 0.38 & 0.07 & 0.06 & 0.12 & - & - \\
\hline F18 & 0.33 & 0.07 & 0.52 & 0.13 & - & - \\
\hline F19 & 0.27 & 0.05 & -0.13 & 0.11 & - & - \\
\hline F20 & 0.58 & 0.04 & -0.02 & 0.11 & - & - \\
\hline F21 & 0.51 & 0.05 & -0.00 & 0.17 & - & - \\
\hline
\end{tabular}


Table 3 (Continued)

\begin{tabular}{|c|c|c|c|c|c|c|}
\hline \multirow[t]{2}{*}{$\begin{array}{l}\text { Task } \\
\#\end{array}$} & \multicolumn{2}{|c|}{ General factor } & \multicolumn{2}{|c|}{$\begin{array}{l}\text { Group factor for } \\
\text { gender-stereotyped female text }\end{array}$} & \multicolumn{2}{|c|}{$\begin{array}{l}\text { Group factor for } \\
\text { gender-stereotyped male text }\end{array}$} \\
\hline & $B$ & S.E. & $B$ & S.E. & $B$ & S.E. \\
\hline F22 & 0.54 & 0.06 & 0.14 & 0.16 & - & - \\
\hline $\mathrm{F} 23$ & 0.43 & 0.05 & 0.02 & 0.18 & - & - \\
\hline M1 & 0.27 & 0.05 & - & - & 0.28 & 0.15 \\
\hline M2 & 0.35 & 0.06 & - & - & 0.08 & 0.38 \\
\hline M3 & 0.30 & 0.07 & - & - & 0.06 & 0.46 \\
\hline M4 & 0.63 & 0.06 & - & - & 0.16 & 0.30 \\
\hline M5 & 0.35 & 0.06 & - & - & 0.26 & 0.20 \\
\hline M6 & 0.37 & 0.06 & - & - & 0.06 & 0.16 \\
\hline M7 & 0.57 & 0.05 & - & - & 0.19 & 0.16 \\
\hline M8 & 0.51 & 0.05 & - & - & 0.16 & 0.19 \\
\hline M9 & 0.37 & 0.05 & - & - & 0.27 & 0.49 \\
\hline M10 & 0.63 & 0.05 & - & - & 0.06 & 0.44 \\
\hline M11 & 0.53 & 0.05 & - & - & 0.02 & 0.22 \\
\hline M12 & 0.51 & 0.04 & - & - & 0.05 & 0.17 \\
\hline M13 & 0.41 & 0.05 & - & - & 0.17 & 0.22 \\
\hline M14 & 0.48 & 0.05 & - & - & -0.11 & 0.18 \\
\hline M15 & 0.44 & 0.06 & - & - & 0.01 & 0.28 \\
\hline M16 & 0.42 & 0.06 & - & - & 0.22 & 0.32 \\
\hline M17 & 0.46 & 0.05 & - & - & 0.18 & 0.16 \\
\hline M18 & 0.39 & 0.05 & - & - & 0.01 & 0.29 \\
\hline M19 & 0.66 & 0.04 & - & - & -0.20 & 0.42 \\
\hline M20 & 0.40 & 0.05 & - & - & -0.10 & 0.17 \\
\hline M21 & 0.65 & 0.06 & - & - & -0.19 & 0.51 \\
\hline M22 & 0.32 & 0.05 & - & - & -0.03 & 0.22 \\
\hline M23 & 0.49 & 0.06 & - & - & 0.42 & 0.87 \\
\hline M24 & 0.39 & 0.04 & - & - & 0.04 & 0.14 \\
\hline
\end{tabular}

N1-N21: tasks with gender-neutral text connotation, reference tasks, F1-F23: tasks with female text connotation, $M 1-M 24$ : tasks with male text connotation

$B$ refer to standardized factor loadings; significant loadings are in bold, $p<0.05$

With regard to gender-stereotypical female connotation there was a significant mean difference between women and men $(M=-0.53$ logits, $S D=0.21, p<0.01$; $d=-0.18$ ). However, given that only three (F13, F16, and F18) out of 23 tasks showed significant loadings on the specific factor (see Table 3 ) the observed mean difference did not indicate support for Hypothesis 2. After all, our hypothesis did not refer to individual tasks, but on the gender-stereotypical text connotation.

Therefore, the expected interaction between reader characteristics (gender) and text characteristics (gender-stereotypical text content) in hypothesis 2 was not confirmed. 


\section{Discussion}

\subsection{Summary}

The main goal of the study was to examine whether gender-stereotypical content in texts making up a reading competence assessment function as an additional dimension of reading competence and explain gender differences in this key competence.

The analyses confirmed a general factor for reading competence at the text level. However, no substantial additional variance was explained by the two hypothesized gender-specific group factors for reading competence. Although prior research has identified stable, socially shared gender stereotypes about typically female and typically male attributions, behaviors and interests (Christin 2012; Diekman and Eagly 2000; Fiske et al. 2002; Haines et al. 2016), which are reflected, for example, in the gender segregation on the labor market (OECD 2013a) and in leisure time activities (e.g., Christin 2012; Lagaert et al. 2017), the findings showed that genderstereotyped text material in a reading assessment has no impact on reading competence. We assumed on the basis of previous research that gender stereotypes might be activated through text content and thus linked to individuals' prior knowledge and experiences with different topics, increasing their motivation to work with such texts. However, our study could not confirm an effect of gender-stereotypical text content on women's and men's performance in a reading competence assessment.

It is possible that gender stereotypes at the broader level of reading assessment texts have more complex effects on reading competence. McElvany et al. (2016) demonstrated small qualitative gender differences in vocabulary among primary schoolchildren at the hierarchically lower level of words. Children more often knew words consistent with stereotypes associated with their own gender than words associated with the other gender group. Thus, gender stereotypes could be transmitted via different mechanisms, especially in adults, who have a wider range of prior knowledge. Future research could conduct a deeper analysis of text structure-for example, by examining storylines - to disentangle the activation of gender stereotypes through written material (cf. studies regarding grammatical gender representations: Garnham et al. 2012).

Moreover, more complex texts that challenge readers' motivation to work on the reading assessment tasks might be relevant for disentangling the expected effect. Even though our results also found no effect of gender-stereotypical text content when restricted to only difficult items, more complex texts overall might enhance the effect of potential mechanisms for the activation of gender stereotypes (cf. moderators of stereotype threat effect; Martiny and Götz 2011). An experimental setting would provide further opportunities to test similar research hypotheses regarding gender stereotypes in text content. Additionally, it might be worthwhile to examine information about individuals' prior knowledge and familiarity with the text or motivation during the assessment by means of additional questionnaire items or incidental data logged during the computer-based assessment itself. Future research could exploit these data to draw inferences about individuals' process strategies by analyzing jumps between texts and tasks or the time readers spend on texts or tasks. 
Concerning the second hypothesis at the individual level, the findings revealed a lack of significant gender differences in the gender-specific factors for reading competence, mostly because the empirical data best fit a unidimensional model with a general latent factor for reading competence only. Women and men were equally competent in reading regardless of potential gender stereotypes in the texts they read. Thus, this study's results were in line with findings from PIAAC, which also reported no substantial competence differences between women and men (OECD 2016a). However, it should be noted that reading abilities as measured in PIAAC do not refer to the same reading comprehension framework as large-scale school-based assessments such as PISA (Lundetræ et al. 2014). Nevertheless, the results from PIAAC and our study indicate that the reading competences of adult women and men are equal and that the gender differences often found in childhood and adolescence seem to decrease over time. A longitudinal analysis of gender differences in reading competence between adolescence and adulthood is necessary to further describe and explain why and how gender differences in this competence domain fade out.

Moreover, a future study could ask a similar question for a younger sample. Since gender differences in reading competence remain constant through adolescence (e.g., Berendes et al. 2018; Solheim and Lundetræ 2017; Weis et al. 2019), it would be interesting to investigate the effect of gender-stereotypical text content in explaining gender differences in reading competence among this age group.

\subsection{Limitations}

The current study extended previous research on the assessment of reading competence and gender differences in reading competence in a sample of adults. Nevertheless, the study also has some limitations.

First, the participants' age was not representative for the general population of adults in Germany due to an oversampling of university students in this sample. The sample's level of education was also very high on average compared to the German population for this same reason. Research findings indicate that gender stereotypes are related to age and education (e.g., Athenstaedt 2000). It is possible that a younger, more educated sample such as the one in our study may endorse slightly different stereotypes of women and men than a more representative sample regarding age and education, which might have had potential effects on our study findings. However, this means that we might be underestimating the effect in the general population of adults.

Second, the study did not include an explicit measure of whether the texts were perceived as gender-stereotypical by the participants. The current study is based on previous research on gender stereotypes concerning women's and men's experiences, prior knowledge and interest in various domains (e.g., Athenstaedt 2009; Christin 2012; Heilman 2012; Lagaert et al. 2017; OECD 2013a). We applied the same argument to our study by having experts rate the presence of gender stereotypes in the text material. Future research might also add participant ratings to further investigate the individual endorsement of stereotypes and possible mechanisms.

Third, no additional information about participants' occupations, university majors, or leisure time activities were available in this pilot study. This is problematic 
because we were not able to include individuals' actual experiences or interests in diverse areas into the model. Collecting data on participants' occupations or leisure time activities may enable future research to control for diverse gender-stereotypical interests or experiences.

\section{Conclusion}

In conclusion, the current study examined whether gender-stereotypical content in stimulus texts within a reading competence assessment distorts competence measurements and serves as a nuisance factor resulting in gender differences in adults' reading competence.

Gender differences in reading competence have been repeatedly identified. Specifically, adolescent girls are more competent readers than boys (OECD 2019). In adulthood, gender differences seem to vanish (OECD 2016a). Previous findings confirm the presence of consistent and long-lasting gender stereotypes about women and men (e.g., Diekman and Eagly 2000; Haines et al. 2016; Lagaert et al. 2017; OECD 2013a). There is also evidence that language transmits gender-stereotyped attributions and characteristics through representations of words and text processing (e.g., Garnham et al. 2016). However, this study found no evidence that gender-stereotypical text content explains interindividual differences in a reading competence assessment. In light of these results, gender-stereotypical text content appears to be negligible to explain gender differences in adults' reading competence.

Funding Open Access funding enabled and organized by Projekt DEAL.

Open Access This article is licensed under a Creative Commons Attribution 4.0 International License, which permits use, sharing, adaptation, distribution and reproduction in any medium or format, as long as you give appropriate credit to the original author(s) and the source, provide a link to the Creative Commons licence, and indicate if changes were made. The images or other third party material in this article are included in the article's Creative Commons licence, unless indicated otherwise in a credit line to the material. If material is not included in the article's Creative Commons licence and your intended use is not permitted by statutory regulation or exceeds the permitted use, you will need to obtain permission directly from the copyright holder. To view a copy of this licence, visit http://creativecommons.org/licenses/by/4. $0 /$.

\section{References}

Adams, R. J., Wu, M.L., \& Wilson, M.R. (2015). ACER ConQuest: Generalised item response modelling software (Computer software, Version 4). Camberwell: Australian Council for Educational Research.

Artelt, C., \& Schlagmüller, M. (2004). Der Umgang mit literarischen Texten als Teilkompetenz im Lesen? Dimensionsanalysen und Ländervergleiche. In U. Schiefele, C. Artelt, W. Schneider \& P. Stanat (Eds.), Struktur, Entwicklung und Förderung von Lesekompetenz. Vertiefende Analysen im Rahmen von PISA 2000 (pp. 169-196). Wiesbaden: VS.

Artelt, C., Schiefele, U., \& Schneider, W. (2001). Predictors of reading literacy. European Journal of Psychology of Education, 16(3), 363-383.

Athenstaedt, U. (2000). Normative Geschlechtsrollenorientierung: Entwicklung und Validierung eines Fragebogens. Zeitschrift für Differentielle und Diagnostische Psychologie, 21(1), 91-104.

Athenstaedt, U. (2003). On the content and structure of the gender role self-concept: Including genderstereotypical behaviors in addition to traits. Psychology of Women Quarterly, 27(4), 309-318.

Athenstaedt, U., Mikula, G., \& Bredt, C. (2009). Gender role self-concept and leisure activities of adolescents. Sex Roles, 60(5-6), 399-409. 
Baron, R.A., Markman, G.D., \& Hirsa, A. (2001). Perceptions of women and men as entrepreneurs. Evidence for differential effects of attributional augmenting. Journal of Applied Psychology, 86(5), 923-929.

Becker, M., \& McElvany, N. (2018). The interplay of gender and social background: A longitudinal study of interaction effects in reading attitudes and behaviour. The British journal of educational psychology, 88(4), 529-549.

Berendes, K., Becker, M., Jacoby, J., Flunger, B., Nagengast, B., \& Trautwein, U. (2018). Individuelle Entwicklungsverläufe beim Lesen. Macht das Geschlecht den Unterschied? Zeitschrift für Entwicklungspsychologie und Pädagogische Psychologie, 50(4), 192-208.

Birnbaum, A. (1968). Some latent trait models and their use in inferring an examinee's ability. In F. M. Lord, M.R. Novick \& A. Birnbaum (Eds.), Statistical theories of mental test scores (pp. 397-479). Reading: Addison-Wesley.

Blossfeld, H.-P., Roßbach, H.-G., \& von Maurice, J. (Eds.). (2011). Education as a lifelong process. The German National Educational Panel Study (NEPS) (Zeitschrift für Erziehungswissenschaft: 14. Sonderheft). Wiesbaden: VS.

Chen, F. F., Hayes, A., Carver, C. S., Laurenceau, J.-P., \& Zhang, Z. (2012). Modeling general and specific variance in multifaceted constructs: A comparison of the bifactor model to other approaches. Journal of Personality, 80(1), 219-251.

Christin, A. (2012). Gender and highbrow cultural participation in the United States. Poetics, 40(5), 423-443.

Christmann, U., \& Groeben, N. (1999). Psychologie des Lesens. In B. Franzmann, K. Hasemann, D. Löffler \& E. Schön (Eds.), Handbuch Lesen (pp. 145-223). München: Saur.

Diekman, A. B., \& Eagly, A.H. (2000). Stereotypes as dynamic constructs: women and men of the past, present, and future. Personality and Social Psychology Bulletin, 26(10), 1171-1188.

Eagly, A.H., Nater, C., Miller, D.I., Kaufmann, M., \& Sczesny, S. (2020). Gender stereotypes have changed. A cross-temporal meta-analysis of U.S. public opinion polls from 1946 to 2018. The American psychologist, 75(3):301-315. https://doi.org/10.1037/amp0000494.

Eccles, J. S. (1987). Gender roles and women's achievement-related decisions. Psychology of Women Quarterly, 11(2), 135-172.

Fiske, S. T., Cuddy, A. J. C., Glick, P., \& Xu, J. (2002). A model of (often mixed) stereotype content: competence and warmth respectively follow from perceived status and competition. Journal of Personality and Social Psychology, 82(6), 878-902.

Garnham, A., Gabriel, U., Sarrasin, O., Gygax, P., \& Oakhill, J. (2012). Gender representation in different languages and grammatical marking on pronouns: When beauticians, musicians, and mechanics remain men. Discourse Processes, 49(6), 481-500.

Garnham, A., Oakhill, J., von Stockhausen, L., \& Sczesny, S. (2016). Editorial: Language, cognition, and gender. Frontiers in Psychology, 7, 772.

Gehrer, K., Zimmermann, S., Artelt, C., \& Weinert, S. (2013). NEPS framework for assessing reading competence and results from an adult pilot study. Journal for Educational Research Online, 5(2), 50-79.

Gehrer, K., Zimmermann, S., Artelt, C., \& Weinert, S. (2012). The assessment of reading competence (including sample items for grade 5 and 9). Scientific Use File 2012, Version 1.0.0. Bamberg. https:// www.neps-data.de/Portals/0/NEPS/Datenzentrum/Forschungsdaten/SC4/1-0-0/com_re_2012_en. pdf. Zugegriffen: 19.06.2020.

Graesser, A.C., Singer, M., \& Trabasso, T. (1994). Constructing inferences during narrative text comprehension. Psychological Review, 101(3), 371-395.

Haines, E. L., Deaux, K., \& Lofaro, N. (2016). The times they are a-changing ... or are they not? A comparison of gender stereotypes, 1983-2014. Psychology of Women Quarterly, 40(3), 353-363.

Heilman, M.E. (2012). Gender stereotypes and workplace bias. Research in Organizational Behavior, 32 , $113-135$.

Kintsch, W. (1998). Comprehension. A paradigm for cognition. Cambridge: Cambridge University Press.

Kleinke, K., Schlüter, E., \& Christ, O. (2017). Strukturgleichungsmodelle mit Mplus. Eine praktische Einführung (2nd edn.). Berlin: De Gruyter Oldenbourg.

Lafontaine, D., \& Monseur, C. (2009). Gender gap in comparative studies of reading comprehension: to what extent do the test characteristics make a difference. European Educational Research Journal, $8(1), 69-79$.

Lagaert, S., van Houtte, M., \& Roose, H. (2017). Engendering culture: The relationship of gender identity and pressure for gender conformity with adolescents' interests in the arts and literature. Sex Roles, $77(7-8), 482-495$. 
Logan, S., \& Johnston, R. (2009). Gender differences in reading ability and attitudes: examining where these differences lie. Journal of Research in Reading, 32(2), 199-214.

Lundetræ, K., Sulkunen, S., Gabrielsen, E., \& Malin, A. (2014). A comparison of PIAAC and PISA results. In A. Malin (Ed.), Associations between age and cognitive foundation skills in the Nordic countries: a closer look at the data (pp. 171-187). Jyväskylä: University of Jyväskylä, Finnish Institute for Educational Research.

Martiny, S.E., \& Götz, T. (2011). Stereotype Threat in Lern- und Leistungssituationen theoretische Ansätze, empirische Befunde und praktische Implikationen. In M. Dresel \& L. Lämmle (Eds.), Motivation, Selbstregulation und Leistungsexzellenz (Talentförderung, Expertiseentwicklung, Leistungsexzellenz, Vol. 9, pp. 153-178). Münster: LIT.

McElvany, N., \& El-Khechen, W. (2015). Mädchenwörter, Jungenwörter - Geschlechtsspezifischer Wortschatz im Grundschulalter? Eine Untersuchung mit Kindern deutscher und anderer Familiensprachen. In H.G. Holtappels, A.S. Willems, M. Pfeifer, W. Bos \& N. McElvany (Eds.), Daten, Beispiele und Perspektiven (Veröffentlichungen des Instituts für Schulentwicklungsforschung: Jahrbuch der Schulentwicklung, Vol. 18, pp. 270-297). Weinheim: Beltz Juventa.

McElvany, N., El-Khechen, W., Schwabe, F., \& Kessels, U. (2016). Qualitative Wortschatzunterschiede zwischen Mädchen und Jungen im Grundschulalter. Zeitschrift für Pädagogische Psychologie, 30(1), 45-55.

McGeown, S.P., Norgate, R., \& Warhurst, A. (2012). Exploring intrinsic and extrinsic reading motivation among very good and very poor readers. Educational Research, 54(3), 309-322.

Möller, J., \& Schiefele, U. (2004). Motivationale Grundlagen der Lesekompetenz. In U. Schiefele, C. Artelt, W. Schneider \& P. Stanat (Eds.), Struktur, Entwicklung und Förderung von Lesekompetenz. Vertiefende Analysen im Rahmen von PISA 2000 (pp. 101-124). Wiesbaden: VS.

Müller, B., \& Richter, T. (2014). Lesekompetenz. In J. Grabowski (Ed.), Sinn und Unsinn von Kompetenzen. Fähigkeitskonzepte im Bereich von Sprache, Medien und Kultur (pp. 29-49). LeverkusenOpladen: Budrich, Barbara.

Muthén, L. K., \& Muthén, B. O. (2017). Mplus user's guide (8th edn.). Los Angeles: Muthén \& Muthén.

Nosek, B. A., Banaji, M.R., \& Greenwald, A. G. (2002). Math = male, me $=$ female, therefore math not $=$ me. Journal of Personality and Social Psychology, 83(1), 44-59.

Nowicki, E. A., \& Lopata, J. (2017). Children's implicit and explicit gender stereotypes about mathematics and reading ability. Social Psychology of Education, 20(2), 329-345.

OECD (2013a). Closing the gender gap: act now. Paris: OECD Publishing.

OECD (2013b). Literacy, numeracy and problem solving in technology-rich environments. Framework for the OECD survey of adult skills. Paris: OECD Publishing.

OECD (2016a). Skills matter: Further results from the survey of adult skills. Paris: OECD Publishing.

OECD (2016b). PISA 2015 results (Volume 1): excellence and equity in education (PISA, Vol. 1). Paris: OECD Publishing.

OECD (2019). PISA 2018 results: what students know and can do. Paris: OECD Publishing.

Pohl, S., \& Carstensen, C.H. (2013). Scaling of competence tests in the National Educational Panel Study-Many questions, some answers, and further challenges. Journal for Educational Research Online, 5(2), 189-216.

Prentice, D. A., \& Carranza, E. (2002). What women and men should be, shouldn't be, are allowed to be, and don't have to be: the contents of prescriptive gender stereotypes. Psychology of Women Quarterly, 26(4), 269-281.

Reise, S.P. (2012). The rediscovery of bifactor measurement models. Multivariate Behavioral Research, 47(5), 667-696.

Rodriguez, A., Reise, S.P., \& Haviland, M. G. (2016). Applying bifactor statistical indices in the evaluation of psychological measures. Journal of Personality Assessment, 98(3), 223-237.

Rost, J. (2004). Lehrbuch Testtheorie - Testkonstruktion (2nd edn.). Bern: Huber.

Schiefele, U., Schaffner, E., Möller, J., \& Wigfield, A. (2012). Dimensions of reading motivation and their relation to reading behavior and competence. Reading Research Quarterly, 47(4), 427-463.

Schwabe, F., McElvany, N., \& Trendtel, M. (2015). The school age gender gap in reading achievement: Examining the influences of item format and intrinsic reading motivation. Reading Research Quarterly, $50(2), 219-232$.

Solheim, O.J., \& Lundetræ, K. (2017). Can test construction account for varying gender differences in international reading achievement tests of children, adolescents and young adults? - A study based on Nordic results in PIRLS, PISA and PIAAC. Assessment in Education: Principles, Policy \& Practice, $3,1-20$. 
Stanat, P., \& Kunter, M. (2002). Geschlechterspezifische Leistungsunterschiede bei Fünfzehnjährigen im internationalen Vergleich. Zeitschrift für Erziehungswissenschaft, 4(1), 28-48.

Steffens, M.C., \& Jelenec, P. (2011). Separating implicit gender stereotypes regarding math and language: Implicit ability stereotypes are self-serving for boys and men, but not for girls and women. Sex Roles, 64(5-6), 324-335.

Watson, A., Kehler, M., \& Martino, W. (2010). The problem of boys' literacy underachievement. Raising some questions. Journal of Adolescent \& Adult Literacy, 53(5), 356-361.

Weis, M., Doroganova, A., Hahnel, C., Becker-Mrotzek, M., Lindauer, T., Artelt, C., \& Reiss, K. (2019). Lesekompetenz in PISA 2018 - Ergebnisse in einer digitalen Welt. In K. Reiss, M. Weis, E. Klieme \& O. Köller (Eds.), PISA 2018. Grundbildung im internationalen Vergleich (1st edition., pp. 47-80). Münster: Waxmann.

Wigfield, A., Eccles, J.S., Roeser, R.W., \& Pamela, D.-K. (2006). Development of achievement motivation. In N. Eisenberg \& W. Damon (Eds.), Social, emotional, and personality development (6th edition Handbook of child psychology, Vol. 3, pp. 933-1002). Hoboken: Wiley. 